\title{
Design, Control, and Implementation of a Robotic Gait Rehabilitation System for Overground Gait Training
}

by

\author{
Aliasgar Morbi \\ A Dissertation submitted to \\ the Faculty of Graduate Studies and Research \\ in partial fulfilment of \\ the requirements for the degree of \\ Doctor of Philosophy \\ Ottawa-Carleton Institute for \\ Mechanical and Aerospace Engineering
}

Department of Mechanical and Aerospace Engineering

Carleton University

Ottawa, Ontario, Canada

December 19, 2013

Copyright (C)

2014 - Aliasgar Morbi 
The undersigned recommend to

the Faculty of Graduate Studies and Research acceptance of the Dissertation

\title{
Design, Control, and Implementation of a Robotic Gait Rehabilitation System for Overground Gait Training
}

\author{
Submitted by Aliasgar Morbi \\ in partial fulfilment of the requirements for the degree of \\ Doctor of Philosophy
}

\begin{tabular}{c}
\hline M. Ahmadi, Supervisor \\
\hline M. Kermani \\
\hline D. Spinello \\
J. Hayes \\
\hline A.D.C. Chan \\
\hline S. Ulrich
\end{tabular}

M. Yaras, Department Chair 
Carleton University

2014 


\section{Abstract}

Robotic devices for gait rehabilitation have the potential to improve patient and caregiver safety, reduce therapy costs, and allow a larger number of patients to get access to physical therapy. Additionally, data collected from the robot's sensors may be used to assess impairment severity and track patient progress. However these devices also suffer from many drawbacks such as high cost, complexity, limited training capabilities, and constrained joint motions and postural responses. With these limitations in mind, this thesis introduces GaitEnable, a simply designed robotic gait trainer that combines an intelligent reactive controller, an actuated omnidirectional mobile base and a passive body weight support system. In addition to describing the device and its control system, this thesis also presents results from a series of validation experiments performed to characterize the performance of the device. The results demonstrate that GaitEnable's control system ensures stable humanrobot interactions, and that GaitEnable can assist and perturb a user's gait in a systematic manner. The experiments also confirm that GaitEnable's actuated omni-directional mobile allows users to walk more naturally as it reduces the motion constraints that the device imposes.

In addition to describing the GaitEnable system, this thesis also focuses on the more general problem of interaction stability in coupled human-robot systems. Two novel trajectory manipulations for ensuring stable, oscillation-free interactions in admittance-controlled haptic devices are proposed. A Lyapunov stability analysis is used to show that the proposed manipulations are stable in the sense of uniform ultimate boundedness. Also, an extensive set of experiments confirm that the impedance manipulations allow the display of large apparent inertia reductions, ensure stable interactions, and are robust to actuator 
saturation and model uncertainties. These features make them ideal for use with robotic systems that are attached to humans (e.g., the GaitEnable system). Use of these manipulations is also extended to typical position control problems, and additional experimental results confirm the manipulations help eliminate chatter and provide a better transient response and steady-state tracking error. 
To my parents. Your sacrifices allowed me to have this opportunity.

To my dear wife. Thank you for giving me a chance to live my dreams. I can't wait to make new ones with you. 


\section{Acknowledgments}

I would like to thank my supervisor Dr. Mojtaba Ahmadi for his mentorship, guidance, and friendship over the many years I spent at Carleton. Whenever I was stuck, I always knew that I could knock on his door and immediately be invited in. More than that, I will be forever grateful to him for affording me the freedom to pursue what I was most passionate about. Opportunities like that are rare, and I'm thankful that he had enough faith in me to let me explore without boundaries.

I also want to thank the staff in the MAE office and at the machine shop for their assistance with the multitude of requests I inevitably came to them with over the years. Finally, I also want to thank the numerous lab mates who I've had the pleasure of spending time with, and being friends with over the last five years. It was a long road to get here, and your suggestions, help and company made it possible. 


\section{Table of Contents}

$\begin{array}{ll}\text { Abstract } & \text { ii }\end{array}$

Acknowledgments $\quad$ v

Table of Contents vi vi

List of Tables $\quad x$

List of Figures $\quad$ xi

List of Abbreviations $\quad$ xiv

1 Introduction 1

1.1 Motivation . . . . . . . . . . . . . . . . . 2

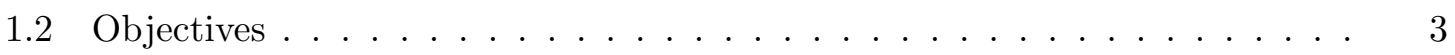

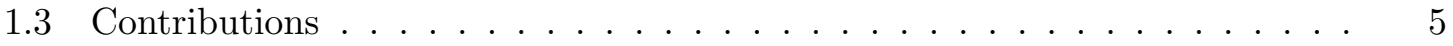

1.4 Thesis Overview . . . . . . . . . . . . . . . . . . 7

2 Background 10

2.1 Body-weight Supported Treadmill Training and the Advent of Gait Rehabilitation Robotics . . . . . . . . . . . . . . . . . . 10

2.2 Principles to Guide the Design of Robotic Gait Rehabilitation Devices . . . 12

2.3 Review of Existing Robotic Rehabilitation Technologies . . . . . . . . . . 19

2.3.1 End-effector-type Gait Rehabilitation Robots . . . . . . . . . . . . 20

2.3.2 Exoskeleton-type Rehabilitation Robots . . . . . . . . . . . 23 
2.3.3 Actuated Mobile Gait Trainers . . . . . . . . . . . . . . . 25

2.3.4 Human Augmentation Exoskeletons . . . . . . . . . . . 26

2.4 The Bungee Mobility Trainer . . . . . . . . . . . . . . . . . 27

2.5 Facilitating Physical Human-Robot Interactions via Admittance Control . . 31

2.5.1 Comparing Impedance and Admittance Control . . . . . . . . . . . . 33

2.5.2 Example Implementation of an Admittance Controller . . . . . . . 35

2.5.3 A Brief Review of Impedance and Admittance Control for Haptics 37

3 Stable Interactions in Admittance-controlled Haptic Devices 41

3.1 Admittance Control of Haptic Devices . . . . . . . . . . . . . . . . 42

3.1 .1 Haptic Device Dynamics . . . . . . . . . . . . . . . . . . . 42

3.1.2 Target Impedance and Reference Trajectory Generation . . . . . . . 43

3.1.3 Joint Space PD Control Formulation . . . . . . . . . . . . . . . . . 44

3.1.4 Task Space PD Control Formulation . . . . . . . . . . . . . 45

3.2 Position Control Loop Stability . . . . . . . . . . . . . . . . . 46

3.2 .1 Problem Statement . . . . . . . . . . . . . . . 46

3.2.2 Guaranteeing the Stability of the Joint-Space PD Controller . . . . . 47

3.2.3 Guaranteeing the Stability of the Task-Space PD Controller . . . . . 51

3.3 Oscillation Attenuation at Low Apparent Inertia Settings . . . . . . . . . 54

3.3.1 Error Feedback in the Target Impedance . . . . . . . . . . . . 56

3.3.2 Extension to Task-space Admittance Control . . . . . . . . . . . . 58

3.4 Experimental Results with a 1-DOF Haptic Device . . . . . . . . . . . . 59

3.4.1 Experimental Apparatus . . . . . . . . . . . . . . . . . 60

3.4 .2 Implementation Details . . . . . . . . . . . . . . . . . . 61

3.4.3 Bounding Tracking Errors by Limiting the Reference Acceleration . 61

3.4.4 Stability Under Actuator Saturation . . . . . . . . . . . . 65

3.4.5 Oscillation Attenuation via Error Feedback . . . . . . . . . . . . 69

3.4.6 Stable Interactions during Dynamic Device Motions . . . . . . . . . 74

3.4.7 Experiments with Multiple Healthy Subjects . . . . . . . . . . . 78 
3.4.8 Comparison with Different Interaction Controllers . . . . . . . . . . 81

3.5 Extension to Position Control Applications . . . . . . . . . . . . . 84

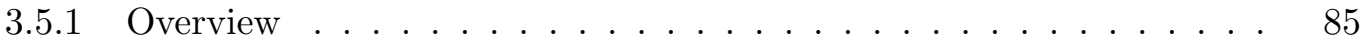

3.5.2 Tracking Error Bounds . . . . . . . . . . . . 87

3.5.3 Comparing the Dual-Loop Controller to a Conventional PD Controller 88

3.6 Chapter Summary . . . . . . . . . . . . . . . . . . . . . 97

4 GaitEnable: A Robotic Gait Trainer for Overground Gait Training 100

4.1 Introduction . . . . . . . . . . . . . . . . . . . 100

4.1 .1 Training and Design Requirements . . . . . . . . . . . . . 102

4.1.2 Limitations of the Bungee Mobility Trainer . . . . . . . . . . . 103

4.2 Overview of the GaitEnable Gait Trainer . . . . . . . . . . . . . . 105

4.2 .1 The Mobile Base . . . . . . . . . . . . . . . . . . 107

4.2 .2 The User-Device Interface . . . . . . . . . . . . . . 116

4.3 GaitEnable's Interaction Control System . . . . . . . . . . . . . . . . . 118

4.3 .1 Equations of Motion . . . . . . . . . . . . . . . . 119

4.3 .2 The Interaction Model . . . . . . . . . . . . . . . . . . 121

4.3.3 Decomposing Reference Velocities to Corresponding Omniwheel Ve-

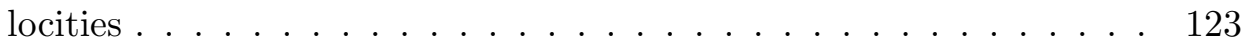

4.3.4 Manipulating Desired Omniwheel Accelerations to Guarantee Stable Interactions . . . . . . . . . . . . . . . . . . . 124

4.3.5 Omniwheel Position Controllers . . . . . . . . . . . . . 126

4.4 Preliminary Experiments . . . . . . . . . . . . . . 127

4.4 .1 Experiment Overview . . . . . . . . . . . . . . . 127

4.4 .2 Results and Discussion . . . . . . . . . . . . . . 128

4.5 The Future of GaitEnable . . . . . . . . . . . . . . . . . . . . . . 132

4.6 Chapter Summary . . . . . . . . . . . . . . . . . . . . 133

5 Experiments with GaitEnable $\quad 136$

5.1 Experiment Objectives . . . . . . . . . . . . . . 136 
5.2 Walking Experiments . . . . . . . . . . . . . . . . 138

5.2 .1 Protocol . . . . . . . . . . . . . . . . . . . . 139

5.2 .2 Data Collection . . . . . . . . . . . . . . . . . . . . 143

5.2 .3 Characterizing Change in Effort . . . . . . . . . . . . . 143

5.2 .4 Results . . . . . . . . . . . . . . . . . 145

5.3 Generating Gait Perturbations via the Admittance Model . . . . . . . . . 157

5.4 Comparing the Force Input Required with and without a Powered Base . . 160

5.5 Investigating the Effects of the Passive Linkage . . . . . . . . . . . . . 161

5.6 Chapter Summary . . . . . . . . . . . . . . . . . . 163

6 Conclusion and Recommendations 165

6.1 Future Work and Recommendations . . . . . . . . . . . . . 167

$\begin{array}{ll}\text { List of References } & 169\end{array}$

$\begin{array}{ll}\text { Appendix A } & 186\end{array}$ 


\section{List of Tables}

3.1 Comparing tracking errors with and without the acceleration limits active . 63

3.2 Reducing oscillations by using error feedback in the reference model . . . . 70

3.3 Dynamic motions at high apparent inertia reduction settings . . . . . . . 77

5.1 Comparing the root mean square values of the encoder- and motion capture system-based reference point velocity estimates . . . . . . . . . . . . 149

5.2 The effective masses and inertias displayed by GaitEnable at 2 different desired mass settings . . . . . . . . . . . . . . . . . . . 152

5.3 Changes in the RMS interaction forces and moments as a result of applying assistance forces through the admittance model. . . . . . . . . . . . 155

5.4 Changes in the RMS interaction forces and moments between omnidirectional and constrained walking. . . . . . . . . . . . 156

5.5 Comparing walking with casters versus walking with the powered base . . . 161

5.6 Comparing walking with and without the passive linkage . . . . . . . . . 162 


\section{List of Figures}

2.1 Example of body weight-supported treadmill training . . . . . . . . . . 11

2.2 A robotic system for body weight-supported treadmill training. . . . . . . . 12

2.3 Different types of rehabilitation robots . . . . . . . . . . . . . . . 21

2.4 The Bungee Mobility Trainer . . . . . . . . . . . . . . . . 28

2.5 A schematic of the BMT's passive BWS mechanism . . . . . . . . . . . 29

2.6 The passive linkage connecting the user's pelvis to the body weight support

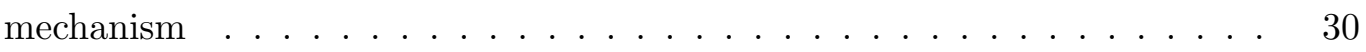

2.7 A block diagram of a generic admittance control structure. . . . . . . . . 35

3.1 A block diagram of the enhanced admittance controller. Bracketed numbers indicate corresponding equation numbers. . . . . . . . . . . . . . . 42

3.2 Experimental results from a 1-DOF haptic device displaying the emergence of limit cycles at consecutively lower inertia reductions. . . . . . . . 55

3.3 A 1-DOF haptic device for assisting elbow motions. . . . . . . . . . . . . 60

3.4 Tracking error response when the user performs abrupt forearm extension at different PD controller gain settings. . . . . . . . . . . . . . . . 64

3.5 Using acceleration limits to eliminate actuator saturation-instigated instabilities. Captions are continued on the following page. . . . . . . . . . . 66

3.5 Using acceleration limits to eliminate actuator saturation-instigated instabil-

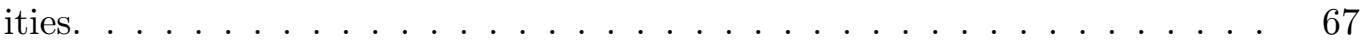

3.6 An image of monitor providing real-time feedback of the desired and measured forearm orientation. . . . . . . . . . . . . . . . 70 
3.7 Safely displaying a 99.3\% apparent inertia reduction. Captions are continued on the following page. . . . . . . . . . . . . . . 72

3.7 Safely displaying a $99.3 \%$ apparent inertia reduction. . . . . . . . . . . 73

3.8 Improved performance during dynamic motions. Captions are continued on

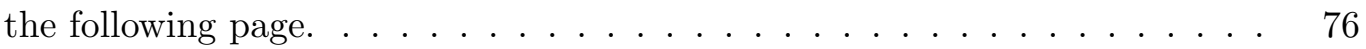

3.8 Improved performance during dynamic motions. . . . . . . . . . . . . . 77

3.9 Rapid extensions performed by healthy subject. . . . . . . . . . . . . 79

3.10 Oscillations caused by a stiff elbow joint. . . . . . . . . . . . . . . 81

3.11 A block diagram of the dual-loop position controller. The impedance manipulations guarantee bounded tracking errors in the inner loop. The user's dynamics can be neglected since this control structure is designed for position control applications. . . . . . . . . . . . . . . 85

3.12 Improved tracking performance during step responses. . . . . . . . . . . . . 90

3.13 Encountering the chattering phenomenon at high position controller gains. . $\quad 92$

3.14 Improved tracking performance during dynamic trajectory tracking. . . . . 94

3.15 Tuning the outer loop to improve the transient response. . . . . . . . . . . 95

4.1 An image of the Bungee Mobility Trainer. . . . . . . . . . . . . . . . . . . 104

4.2 Comparing GaitEnable to different types of rehabilitation robots. . . . . . . 106

4.3 An image of the first prototype of the GaitEnable system with its key components highlighted. . . . . . . . . . . . . . . 108

4.4 An image of the different Rotacaster omniwheels tested on GaitEnable. . 110

4.5 An top-view of the mobile highlighting the wheel positions and orientations. 111

4.6 An image of an assembled motor assembly. . . . . . . . . . . . . . . 112

4.7 A high level overview of the robotic system implemented on GaitEnable. . . 113

4.8 A one-sided interface connected to the passive linkage. . . . . . . . . . . 117

4.9 A multi-lateral interface connected to the passive linkage. . . . . . . . . . 118

4.10 A multi-lateral interface connected to the robot frame. . . . . . . . . . . 119

4.11 A block diagram of the admittance controller. . . . . . . . . . . . . . 120

4.12 The top view of the mobile base. . . . . . . . . . . . . . . . 121 
4.13 Comparing controller performance with and without error feedback (EF) in the reference model. . . . . . . . . . . . . . . . . . . . . . 129

4.13 Comparing controller performance with and without error feedback (EF) in the reference model. . . . . . . . . . . . . . . . . . . . . 130

4.14 An image of the second-generation prototype of the GaitEnable gait trainer. 134

5.1 The markers placed on the floor to specify the sequence of steps to be taken. 140

5.2 The relationship between assistance force and the force the user applies to the user-device interface. . . . . . . . . . . . . . . . . . . . . . 142

5.3 Comparing the reference point velocity estimated from the encoders to the velocity measured from the motion capture system. . . . . . . . . . . . . . 147

5.3 Comparing the reference point velocity estimated from the encoders to the velocity measured from the motion capture system. . . . . . . . . . . . 148

5.4 Sample results from walking experiments from one participant. . . . . . . 150

5.5 A box plot describing the variation of $W$ at the different test conditions. . . 151

5.6 Low speed vibrations generated by omniwheels. . . . . . . . . . . . . 154

5.7 Different reactions to lateral disturbances from GaitEnable. . . . . . . . . . 159

5.8 Comparing the fore/aft interaction force with casters versus the powered mobile base. . . . . . . . . . . . . . . . . . . . . . . 162 


\title{
List of Abbreviations
}

\author{
AL Acceleration Limits \\ BWS Body weight support \\ BWSTT Body weight supported treadmill training \\ CoM Centre of mass \\ D Derivative \\ DOF Degrees-of-freedom \\ P Proportional \\ PC Personal computer \\ PD Proportional derivative \\ PSD Power spectral density \\ RMS Root mean square \\ UUB Uniform ultimately bounded
}




\section{Chapter 1}

\section{Introduction}

There are more than 50,000 ischemic and hemorrhagic strokes in Canada each year [1] - one occurring every 10 minutes - that leave nearly 300,000 Canadians suffering from various symptoms. On average, $50 \%$ of this population suffers from life-long gait impairments. In addition, there are tens of millions individuals worldwide who suffer from different neurological illnesses such as Parkinson's disease, multiple sclerosis, or cerebral palsy. Many of these individuals are unable to perform the activities of daily living and are at a higher risk of falls and injuries when mobilizing.

Reduced lateral stability, the inability to bear load through a limb, and the inability to generate sufficient muscle forces, rank among the most common sensorimotor deficits that contribute to reduced locomotor ability [2]. Focused and repetitive gait rehabilitation therapy has been proven to encourage functional recovery, minimize a patient's need for long-term physical assistance, and improve patient quality of life $[3,4]$. However, therapistassisted overground gait training is physically demanding - therapists may sometimes be required to lift, move and support patients nearly three times their size. Therapist fatigue is also of concern because it limits the intensity and duration of the physical therapy that severely impaired patients receive. Moreover, physical therapy puts patients at risk for

falls and injuries. Thus, these challenges result in many patients receiving an inadequate amount of physical therapy. The work described in this thesis was conceived with the goal of creating an affordable, practical, and versatile robotic gait rehabilitation system that 
improves patient safety and reduces therapist workload.

\subsection{Motivation}

Immobility can lead to accelerated bone and muscle loss, sensory deprivation, isolation, delerium and incontinence [5]. Short bouts of immobility, e.g., even 5-10 days of bed rest during a hospitalization, can contribute to an increased risk of mortality and significant impairments in an individual's long-term ability for self-care and locomotion [6]. Many of these hazards can be avoided by timely and sufficient gait rehabilitation therapy [6-10]. Fundamentally, the key to faster recovery is to walk early, typically within 24-48 hours of a major surgery or acute illness. Many studies show that the simple act of walking 15-25 minutes a day within 48 hours of an acute illness or surgery can improve outcomes and reduce hospitalization stays by $2-5$ days [6-10]. Therefore, tools and practices that encourage users to walk earlier during their hospital stay are critical.

The impact of early gait rehabilitation is substantial since over 5,000,000 orthopaedic, post-surgical and acute cardiovascular, respiratory, and neurological inpatients can benefit from this therapy [11]. While the benefits are clear, two key barriers limit the widespread practice of early mobilization: $i$ ) patient and caregiver safety concerns; and $i i$ ), staffing limitations. Physical and cognitive impairments caused by an illness or medication/sedation can leave many patients at a heightened risk for falls. These falls are a serious problem since nearly $23 \%$ of falls result in serious injuries such as hip fractures [12]. Additionally, serious falls can increase patient care costs by nearly $\$ 35,000$ and result in millions in litigation costs [12]. Accordingly, tools that facilitate early mobility must be designed to prevent falls and reduce a patient's risk of injury during their training.

Caregiver injuries also pose an impediment to the delivery of gait rehabilitation therapy. Support staff such as nurses, nurses' aides, and attendants have the highest 
number of work-related injuries requiring days away from work. Workers compensation costs arising from these injuries can exceed $\$ 7.4$ billion dollars annually [13]. Moreover, bariatric patients and patients with severe mobility impairments can require the assistance of 2-4 staff members to ambulate overground. Staffing limitations, and the challenges in coordinating the schedules of multiple staff mean that many patients are unable to receive sufficient therapy. This problem is particularly serious since severely impaired patients who are difficult to mobilize are likely to suffer the greatest long-term consequences from remaining immobile [6]. Thus, tools that facilitate early walking must also be designed to reduce therapist workload, and the number of therapists required to administer the therapy.

In short, immobility hazards are substantial and numerous. Fortunately, physical therapy and overground gait practice can protect patients from these hazards [6-10]. Given the number of patients that can benefit from the simple activity of walking alone, finding a solution for the challenges that limit patient access to timely and adequate overground gait rehabilitation therapy is imperative. Appropriately designed robotic devices can help address these challenges. Such devices can allow frail patient to safely mobilize earlier during their recovery if: i) they are easy for patients and therapists to use, iii) reduce the number of caregivers required to mobilize a patient, and, iii) are capable of automatically detecting and preventing falls.

\subsection{Objectives}

The need to reduce therapist workload during gait rehabilitation therapy has prompted many researchers to investigate the use of robotic devices for automating gait rehabilitation. In addition to reducing therapist workload, robotic devices can increase patient safety, reduce therapy costs, and allow a larger number of patients to get access to physical therapy of a sufficient intensity and duration. Additionally, data collected from the robot's sensors may be used to assess impairment severity and to quantitatively track patient 
progress over the duration of a training program.

Training in functional environments and contexts is essential during gait rehabilitation therapy. This enables patients to build the skills and confidence necessary to ambulate in their homes and perform the activities of daily living. While many gait rehabilitation robots assist patients in emulating limb motions corresponding to a normal gait [14-23], few are explicitly designed to allow practice of overground walking and training of balance skills. Furthermore, many existing rehabilitation robots are too complicated to use, and are designed to replace the therapist rather than aiding them in their work. Also, the cost of many commercially available systems is prohibitively high; systems such as the Lokomat can cost nearly $\$ 1,000,000$ per system. These drawbacks have ultimately limited the widespread use of robotic gait rehabilitation devices [2].

The primary objective of the research was to design, develop, and realize a robotic gait trainer that facilitates overground gait rehabilitation therapy and balance training. Generally speaking, designing such devices is quite challenging as one must simultaneously consider a variety of issues that relate to usability, safety, ergonomics, and regulatory guidelines. Issues such as patient and caregiver perceptions about the size and look of the device, space constraints within a hospital, the degree of technology aversion that many caregivers exhibit, specific training needs of various patient populations, safety requirements that standards such as the ISO 10535 or IEC 60601 standards impose, and the differing requirements that nurses, doctors, and physiotherapist may have for the device must all be considered. With these many diverse issues at hand, the scope of this research was restricted to:

1. Identifying the primary training and therapy requirements that should guide the design of the device.

2. Designing a minimal robotic system that can satisfy the therapy requirements.

3. Integrating the necessary controllers, sensors, and actuators and developing a prototype suitable for user testing. 
4. Conducting experiments with healthy participants to validate the performance of the robotic system and its human-robot interaction control system.

As with all haptic devices, the design of the human-robot interaction control system had to be completed in tandem with the electromechanical design of the gait trainer. Accordingly, another important focus area of this research included the design, implementation, and experimental evaluation of the gait trainer's interaction control system. Briefly, the key requirements that guided the design of the control system included:

1. Guaranteed stability under all operating conditions.

2. Automated synchronization of the user's and gait trainer's motions.

3. The ability to mask the inertial properties of the gait trainer.

4. The ability to generate desired force cues at the user-device interface.

Since this research explored a novel user-device interface for controlling the gait trainer, the final objective of the research included a comprehensive experimental evaluation of the interaction control system and the gait trainer's performance.

\subsection{Contributions}

The major contributions presented in this dissertation include:

1. Design of a Mobile Gait Trainer: The concept of GaitEnable was developed via a critical review of the state of the art, and an analysis of the key training and therapy requirements that robotic gait trainers must satisfy. GaitEnable, a simple and versatile robotic gait trainer that combines a passive body-weight support system, a novel interaction system and an omniwheel-actuated omnidirectional mobile base, was developed to support the research. GaitEnable is unique with respect to other 
robotic gait trainers because it uses an individual's pelvis as the primary control interface, and because its mobile base is purposely designed to transmit force cues to a user.

2. Implementation and Integration of Prototype: GaitEnable's robotic elements were designed, manufactured and installed on an existing mobile body-weight support system. The integrated prototype was approved for experiments with healthy individuals and patients by the Research Ethics Committee at Carleton University.

3. Introduction and Validation of a Novel Stabilizing Controller for Admittance-Controlled Haptic Devices: A minimal and model-free approach to shaping the admittance and limiting the desired acceleration of a haptic device for ensuring stable user-device interactions in admittance-controlled haptic devices was developed. This methodology was implemented on GaitEnable and another 1-DOF haptic device. A comprehensive experimental evaluation confirms that the control methodology provided improved performance and robustness.

\section{Experiments to Verify Device and Control System Performance: Exper-} iments performed by healthy subjects, described for the first time, the degree to which a patient's pelvis may be used as an interface for manipulating a robot's

motion. Results demonstrate that the control system is capable of generating force cues for assisting or destabilizing a user's gait, and displaying a significantly reduced apparent mass. Results also show that GaitEnable's holonomic mobile base allows a user to walk more naturally because it does not restrict pelvis translation and rotation. 
These contributions have been recognized in the following publications:

1. A. Morbi and M. Ahmadi. "Stable Interactions in Admittance-controlled Haptic Devices," in IEEE Transactions on Robotics. (Revisions requested).

2. A. Morbi, M. Ahmadi, and A. Nativ. "Preliminary Experiments with an Omnidirectional Mobile Robot for Gait Rehabilitation," in International Journal of Mechatronics and Automation. (Forthcoming).

3. A. Morbi, M. Ahmadi, A.D.C Chan, and R. Langlois. "A Stability-Guaranteed Assist-as-Needed Controller for Powered Orthoses," in IEEE Transactions on Control System Technology. (Forthcoming).

4. A. Morbi, M. Ahmadi, and A. Nativ. "GaitEnable: An omnidirectional robotic system for gait rehabilitation," in the proceedings of the 2012 IEEE International Conference on Mechatronics and Automation. Aug. 2012. Pgs. 936-941. (Finalist for Best Paper Award).

5. A. Morbi, M. Ahmadi, and R. Beranek. "Control System and Device for Patient Assist," PCT/CA2013/050251. Published PCT International Patent Application that is pending National Phase Entry.

\subsection{Thesis Overview}

\section{Chapter 2 - Background}

This chapter describes the main issues that must be considered when designing robotic gait rehabilitation systems, and provides a review of the state of the art. This chapter also 
introduces the Bungee Mobility Trainer, the commercially-available mobile body-weight support system that motivated the conceptual design of GaitEnable, and concludes with a brief introduction to admittance control, the interaction control system used on GaitEnable.

\section{Chapter 3 - Stable Interactions in Admittance-controlled Haptic Devices}

This chapter presents two novel trajectory manipulations that are central to GaitEnable's interaction control system. The first manipulation limits the desired acceleration of a haptic device in order to stabilize its position control loop. The second manipulation shapes the commanded acceleration of a haptic device to selectively attenuate sustained oscillation. Both manipulations combined provide a simple, but effective solution to a fundamental problem in haptics of making an admittance-controlled robot safely display a small apparent mass. An analytical stability proof based on Lyapunov stability theory shows how both manipulations guarantee the stability of a robot's task or joint space position control loop. The chapter concludes with an extensive set of experiments performed with a 1-DOF haptic device for verifying the analytical stability results, and a brief discussion on how the impedance manipulations can be applied in generic position control problems.

\section{Chapter 4 - GaitEnable: A Robotic Gait Trainer for Overground Gait Training}

Chapter 4 introduces GaitEnable, and describes its novel interaction control system and omniwheel-actuated omnidirectional mobile base. This chapter summarizes the primary design motivations, provides descriptions of key subsystems, and discusses the implementation of the control system. The chapter concludes with a description of several experiments performed to investigate the stability of the control system. 


\section{Chapter 5 - Experiments with GaitEnable}

This chapter investigates whether GaitEnable's powered omnidirectional mobile base can reduce motion constraints and generate force cues for influencing a user's gait. Results from experiments performed by seven healthy subjects confirm that GaitEnable's powered mobile base is capable of masking the device's inertial properties, and that the control system is capable of transmitting force cues for assisting or perturbing a user's gait. Additional results also show the value of using a powered mobile base in comparison to using castors, and GaitEnable's ability to minimize motion constraints during free walking.

\section{Chapter 6 - Conclusions and Recommendation}

This chapter summarizes the research and discusses possible improvements and directions for future work. 


\section{Chapter 2}

\section{Background}

This chapter provides a review of the literature related to the training and therapy requirements of robotic gait rehabilitation systems, and presents a critical assessment of existing robotic devices used for gait rehabilitation. This chapter also introduces the Bungee Mobility Trainer, the device that inspired the development of the GaitEnable system. A literature review of admittance control, the human-robot interaction control system used in GaitEnable, concludes this chapter.

\subsection{Body-weight Supported Treadmill Training and the Ad- vent of Gait Rehabilitation Robotics}

Recent research in rehabilitation has shown that training intensity, specificity, repetition, and salience are crucial in controlling the degree and rate of functional recovery $[3,4,24]$. Body-weight supported treadmill training (BWSTT) - a therapeutic invention that involves the use of a harness-based body-weight support system during treadmill walking - was first introduced in the mid 1980's [25]. The premise behind BWSTT is that the unloading provided by the body-weight support system allows individuals with limited muscle forces to practice coordinated stepping movements. Furthermore, BWSTT allows control over training intensity (via control over body-weight unloading), is specific and relevant to the end goal of independent walking, and most importantly, allows for repetitive practice of 
walking-like motions.

A variety of studies have shown that after BWSTT, spinal cord injury and stroke patients can improve their locomotion ability [26-31]. However, the physical demands placed on the therapist during BWSTT have limited its widespread use. During BWSTT, trainers must manipulate the patients pelvis/trunk motion to assist lateral weight shifting, propel the patient's leg to ensure it clears the ground, manually guide the swing foot to ensure a properly timed and located foot landing, and prevent buckling of the stance leg [32]. While the use of a body weight unloading system (typically a harness with a counterweight) can relieve some of the physical demands associated with administering the therapy, it may still be necessary to have up to three trained therapists to treat just a single patient. An example of this can be seen in Figure 2.1.

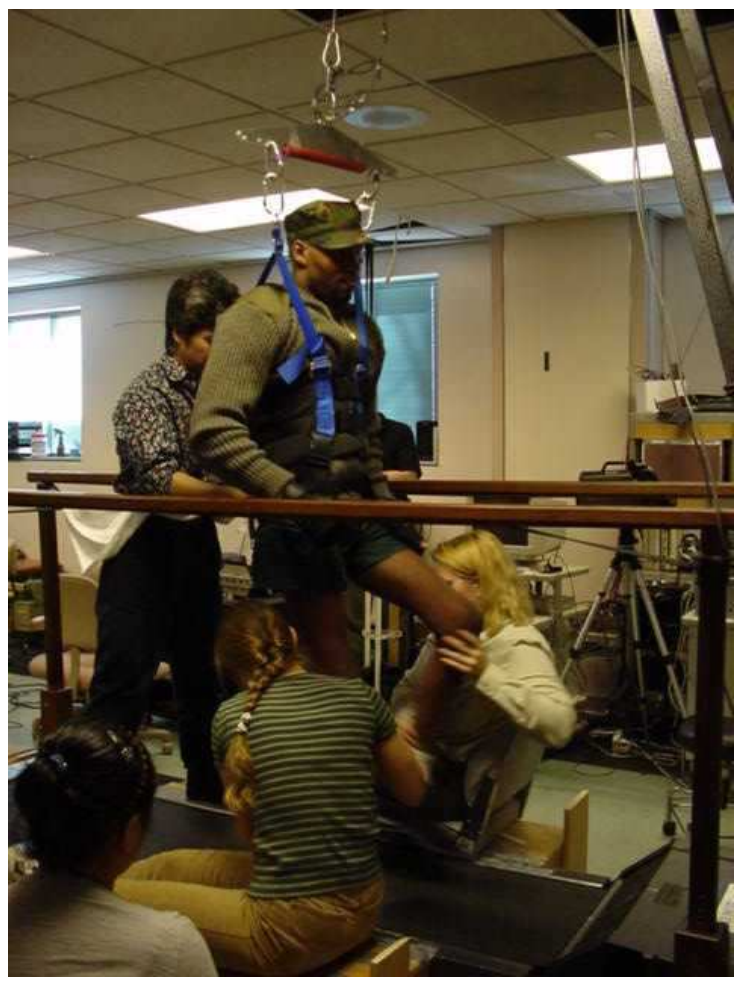

Figure 2.1: Example of body weight-supported treadmill training (from [33]) 
The initial gait rehabilitation robots developed in the late 1990's were designed to address the challenges associated with administering BWSTT $[14,19]$. Colombo and Hesse observed that treadmill-based training was both physically-demanding and repetitive, and hence well-suited to automation. As shown in Figure 2.2, these researchers focussed on designing robotic devices that could keep patients upright and automatically move their lower extremities through motions that were representative of gait. While effective at reducing therapist workload, most of these early devices could not be used for balance training, and did not facilitate training in functional environments and contexts (e.g., walking overground in a hallway). Most importantly though, their widespread use and commercial appeal was limited by their complexity and high cost.

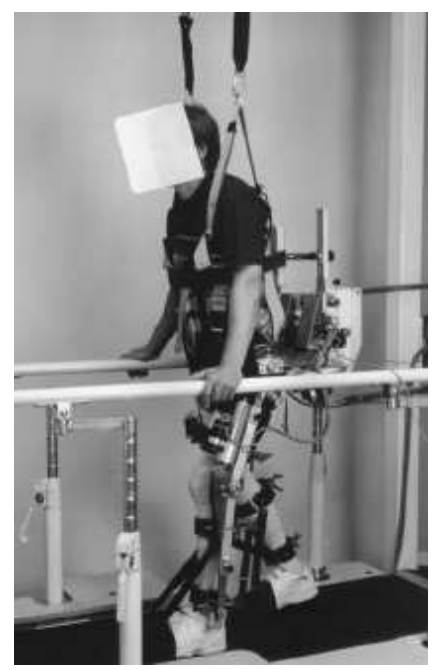

Figure 2.2: A robotic system for body weight-supported treadmill training (from [19])

\subsection{Principles to Guide the Design of Robotic Gait Rehabil- itation Devices}

BWSTT represents just one specific approach to gait rehabilitation. Even though early designs of robotic gait rehabilitation devices were heavily influenced by the requirements of 
this one therapeutic approach, a variety of other issues must be considered when designing these systems. The most important of these issues are identified from the literature and considered below:

\section{- The device should be capable of providing body weight support (BWS)} As noted above, BWS allows patients with limited muscle strength to safely practice coordinated stepping movements. Moreover, several studies have shown that BWSbased gait training strategies yield better clinical outcomes than conventional gait training strategies such as training on parallel bars $[28,34]$. Thus, supporting body weight is an important (safety and performance) feature that should be included in any robotic gait rehabilitation system. However, many researchers opt to use actuated BWS systems that are capable of precisely unloading body weight [35-41]. This choice is motivated by research that suggests that harness-based passive BWS systems using springs and counterweights may greatly disturb the vertical motion of the patient's center of mass, and the nature and timing of the ground contact during BWSTT $[35,38]$. Passive BWS systems also interfere with the processes which govern the exchange of gravitational and kinetic energy during normal gait [39] and are limiting because they provide unnecessary support to the non-paretic limb. However, precise regulation of body weight unloading may be insufficient [42]; normative spatial, temporal, and kinematic gait parameters and physiologically relevant changes in ankle power generation and absorption cannot be achieved even with precise body-weight unloading [42]. As noted in [37] and [42], further research is required to determine the optimal body weight unloading protocols that maximize clinical outcomes. In other words, a BWS system is a crucial and necessary element of any robotic gait rehabilitation system. However, there is still little evidence to suggest that actuated body weight unloading systems are essential to maximizing therapeutic outcomes.

BWS systems are also important for guaranteeing the safety of the patient. Patients 
(and therapists) may be unwilling to perform more challenging exercises and training in the absence of adequate BWS systems that prevent falls. Moreover, BWS systems with controllable body weight unloading enable therapists to gradually and safely increase training challenge and intensity. This is noteworthy since gradually increased training intensity is also essential to promoting functional recovery [24].

\section{- The training that the device provides should closely emulate overground} walking. Task specificity is recognized as one of the fundamental principles that controls experience-dependent neural plasticity, the neural mechanism that contributes to long-lasting functional changes [24]. In the context of gait rehabilitation therapy, this statement implies that a patient is more likely to improve their gait if the training they receive is representative of overground walking. Currently, most rehabilitation robots have been designed to reduce therapist involvement in BWSTT. However, there is no unanimous consensus on the question of how well treadmill walking compares to overground walking (compare [43] and [44] for example). Though studies suggest that BWSTT is more effective than conventional gait rehabilitation techniques $[28,34,45,46]$, these results should not be misconstrued to indicate the supremacy of BWSTT. The key message is that interventions that enable patient mobility (as BWSTT does) result in improved clinical outcomes. Since BWSTT is primarily concerned with motion in the sagittal plane, robotic gait rehabilitation devices that do not constrain (and perhaps even actively control) lateral body motions may prove to be more effective for gait rehabilitation $[47,48]$.

\section{- The device should impose minimal constraints on the patient's motion.} BWSTT and robotic gait rehabilitation systems for BWSTT typically require the use of harness-based BWS systems. However, overhead harnesses constrain and stabilize torso motion through the lateral forces they apply on the patient's torso. These constraints are significant because approximately two thirds of body mass is concentrated above the waist. Hence, torso motion inevitably influences and controls 
many crucial gait characteristics. As noted in [49], changes in average trunk flexion during harness-free gait can cause consistent kinematic adaptations at the hip, knee, and ankle joints, along with corresponding changes in the vertical ground reaction force in heathy individuals. Moreover, results from a recent study indicate that there are significant differences between walking with and without a harness, and between walking on a treadmill wearing a harness (with or without some BWS) [50]. As noted in [50], harnesses and harness-based BWS systems significantly alter trunk acceleration magnitudes and profiles. These results do not imply that harness-based BWS systems have a negative impact; however, they do suggest that harnesses significantly alter certain gait characteristics. In other words, therapies that require the use of harness-based support systems may not faithfully emulate certain features of overground walking.

Pelvis motion also plays a crucial role during gait. Some studies suggest that constraining horizontal pelvis motion during treadmill walking significantly affects the gait of healthy individuals $[47,48]$. Constraining the lateral motion of the pelvis leads to longer and narrower steps, increased step time, excessive trunk rotations, and changes in the vertical ground reaction force [47]. These results do not suggest that constrained pelvis motions have substantial negative impact. Again, they simply suggest that robotic gait rehabilitation systems that constrain pelvis motion may not be capable of emulating normal gait.

As noted in Section 2.3, many rehabilitation robots are equipped with lower extremity exoskeletons for helping guide swing leg motion and preventing stance leg buckling. For the sake of simplicity, many of these exoskeletons are designed to constrain leg motion to the sagittal plane. By constraining ankle rotation and hip adduction/abduction, planar exoskeletons enhance lateral stability and alter the lateral motion of the body center of mass. In fact, results from a study that investigated how 
healthy individuals walk in the Lokomat rehabilitation robot suggest that limiting leg motion to the sagittal plane has a significant impact (i.e., altered gait kinematics and kinetics) on the gait of healthy individuals [51]. Thus, a robotic system designed to emulate normal gait should be designed without imposing such constraints on a user's torso, pelvis, and lower extremities.

- The device should facilitate targeted balance training. Balance is an actively controlled and complex task that requires afferent information from the vestibular system, the visual system, and proprioception [52]. Balance impairments are of particular concern because they are strongly correlated to higher incidences of falls and injury among community-living older adults [53-56].

Recent studies suggest that targeted balance training is effective for improving task-specific balance performance both in healthy seniors [57-60] and stroke patients [55,61-63]. Moreover some systematic reviews also suggest that strength training and gait-oriented training alone do not result in a significant improvement in balance [63-65]. However, as noted in Section 2.3, few robotic gait rehabilitation systems have been designed to facilitate targeted balance training. Given the evidence which suggests the importance and efficacy of balance training, facilitating targeted balance training should also be recognized as a crucial function of any robotic gait rehabilitation device.

A rehabilitation robot's ability to facilitate targeted balance training is strongly linked to the way in which it constrains the patient's body motion. As discussed above, motion constraints artificially enhance the patient's stability and reduce the active effort that the patient needs to exert to maintain balance $[47,48,50,51]$. Thus, effective balance training may not be possible with a robotic gait rehabilitation device that imposes too many unnatural motion constraints. However, a good compromise 
between safety and constraints is also required. As noted later, some forms of motion constraints may be beneficial in the early stages of recovery when a patient is very frail. Thus, a device that can modulate its motion constraints in accordance to the user's recovery would be beneficial.

- The device should allow patients to practice rapid change-in-support reactions and turning while walking. Change-in-support (CIS) reactions include rapid and sudden actions such as a step to the side, the modification of a step in progress, or reaching to grasp an object for support [66]. These reactions have been shown to be more effective than other disturbance compensation methods for stabilizing body motion after a loss of balance [66]. Furthermore, a recent study comparing a perturbation-based balance training program and an exercise program involving flexibility and relaxation training has shown that perturbation-based balance training is more effective in allowing older adults to develop the compensatory reactions necessary to prevent falls [57]. As described in further detail in Section 2.3, many robotic rehabilitation systems limit leg motion to the sagittal plane or force the user's lower extremities through predefined motions. As such, these devices may not be suitable for training CIS reactions. Given the evidence suggesting the effectiveness of targeted balance training $[55,57-60]$ and the prevalence and dangers of falls in the elderly [54], future robotic gait rehabilitation system should specifically be designed to allow perturbation-based training that evokes and trains CIS reactions.

A study of stroke survivors revealed turning while walking to be a common cause of falls in this population group [67]. Similar studies have also revealed turning to be a common cause of falls in Parkinson's disease patients [68]. Moreover, the results from another study suggest that falling while turning increases the risk of hip fractures by a factor of 7.9 in comparison to falling while walking straight ahead [69]. To the author's knowledge, no studies have directly investigated the value and importance of 
turning-specific training in reducing the incidence of falls. However, targeted training of this particular activity may also be effective for reducing the risk of falls associated with this activity.

- The device should minimize the therapist's workload. If the robot reduces or removes the physical demands associated with lifting and supporting the patient, then the therapist is free to focus on treating the patient.

- The device should allow the therapist to manipulate the patient's limbs. Many therapists prefer to directly manipulate a patient's leg during gait rehabilitation therapy [2].

- The device should incorporate a mechanism for helping initiate and sustain the patient's voluntary swing leg motion. Several therapists may be required to assist the patient's swing leg motion in both conventional gait rehabilitation therapy and BWSTT. A robotic gait rehabilitation device capable of guiding or assisting the swing leg motion further reduces the degree of therapist involvement during BWSTT. However, it is important to note that the swing leg assistance mechanism may not be necessary or appropriate for all patients. Therapists may prefer to manually manipulate the patient's legs in some cases. Also, swing leg assistance may not be necessary at later stages in the therapy. Thus, the swing leg assistance mechanism should be easily attached or removed from the device depending on the requirements of the therapy. It is also important to note that the swing leg mechanism should be designed to provide minimal constraints to the patient's hip, knee, and ankle motion.

- The device should require a minimal setup time and should easily adapt to different body morphologies. Some rehabilitation robots can require a 20-30 
minute setup time $[2,20,70]$. Given that some patients may only be able to train for 5-15 minutes per session, long setup times substantially increase the cost of providing the therapy.

- The device needs to be simple and inexpensive. Cost is a key factor that limits the use of robotics in rehabilitation therapy. Systems such as the Lokomat can cost over $\$ 300,000$ per system and require trained staff, dedicated space, and on-going maintenance costs. In contrast, simple mobility devices such as walkers and patient lifts cost between $\$ 500$ and $\$ 15,000$. Clearly, the value a robotic system provides in terms of improvements in patient outcomes and cost savings will determine how much institutions will pay for a device. However, it appears that a price point on the order of $\$ 300,000$ is still prohibitive as only about 600 Lokomat robots have been sold since their launch in 2001. Given the cost of the simpler mobility devices that hospitals currently purchase, it is likely that a price point between $\$ 50,000$ and $\$ 100,000$ may be more appropriate unless these devices are proven to significantly improve patient outcomes/reduce care costs.

Simplicity is similarly crucial since therapists are not trained to use robotic systems. The fear associated with learning how to use these systems alone may prevent their use. Finally, therapists are interested in technologies that enhance their ability to do their work [2]. Treating the therapist as the end-user results in a device design that is more likely to be adopted by a larger number of therapists [2].

\subsection{Review of Existing Robotic Rehabilitation Technologies}

The development of mechanized gait trainers and gait rehabilitation robots is historically rooted to the emerging use of BWSTT as an intervention for stroke and spinal cord patients. In particular, these devices were conceived primarily for reducing therapist workload 
during BWSTT. However, robotic gait rehabilitation devices are also beneficial because they easily facilitate repetitive, task-specific training and because they can be designed to provide a controllable level of assistance (or resistance). Furthermore, they enable the development of new rehabilitation protocols, and the data from their sensors can be used to quantify impairment severity and patient recovery. Most importantly though, by reducing therapist workload and overall therapy cost, rehabilitation robotics have the potential to enable a larger number patients to easily gain access to the physical therapy they require.

Gait rehabilitation robots can be separated into three different categories depending on their function and design: end-effector-type robots that attach to the user's feet and guide the motion of the user's feet [14-18], exoskeleton-type robots that attach parallel to the patient's lower extremities [19-23], and actuated mobile gait trainers that enable overground gait training [71-73]. An example of each type of gait rehabilitation robot platform is shown in Figure 2.3. As with BWSTT, skepticism regarding the effectiveness of robotic gait rehabilitation platforms still exist. However, a recent Cochrane systematic review suggests that patients who receive robot-assisted gait training in combination with physiotherapy after stroke are more likely to achieve independent walking than patients receiving gait training without these devices [74].

\subsubsection{End-effector-type Gait Rehabilitation Robots}

Development of the first end-effector-type robot, now sold commercially as the Gait-Trainer GT I, began in the late 1990's. This device consists of two actuated footplates that guide a patient's feet through a pre-defined motion that simulates gait. The primary advantage of this device is that it enables repetitive practice of gait-like motions without significant therapist involvement [14]. However, the Gait Trainer GT I - like the treadmill-driven LokoHelp robot [16], which has similar capabilities - only simulates walking motions. More advanced end-effector-type robots like the HapticWalker [15] and the recently developed robots by Hesse et. al [17] and Yoon et. al [18], employ actuated footplates capable of 


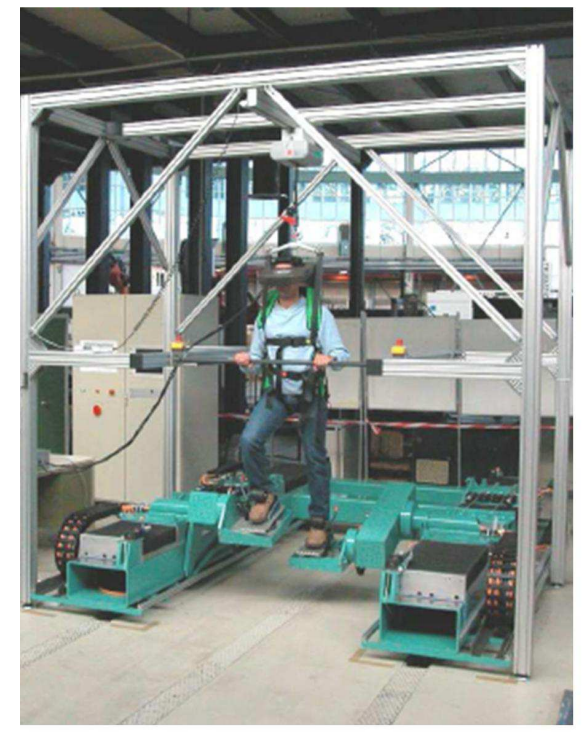

End-Effector Type (Haptic Walker)

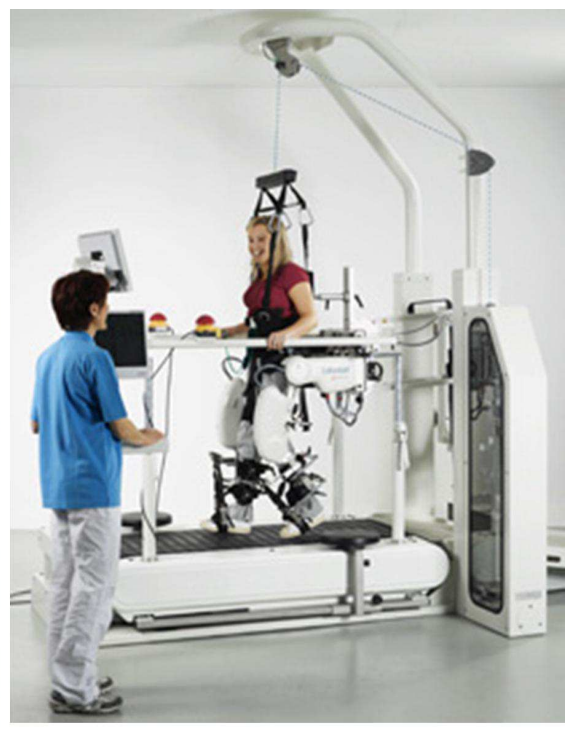

Exoskeleton Type (Lokomat)

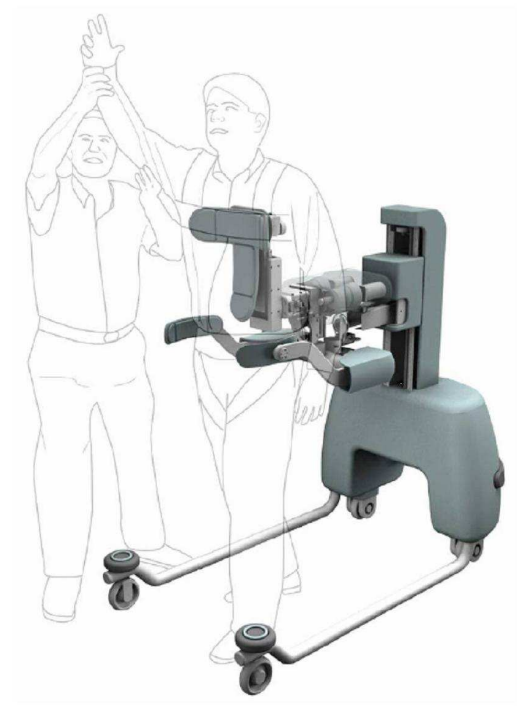

\section{Actuated Mobile Gait Trainers (KineAssist)}

Figure 2.3: Different types of rehabilitation robots 
following arbitrary trajectories. These advanced robots are much more versatile because they allow patients to safely practice activities such as stair climbing and walking on uneven terrain that would otherwise be difficult to practice using other types of gait rehabilitation robots.

Experiments with both healthy subjects and stroke patients have shown that muscle activation patterns between free walking and simulated walking in end-effector type rehabilitation robots are qualitatively similar $[17,75]$. This suggests that the gait practice with an end-effector type rehabilitation robot provides a faithful approximation of overground walking. Furthermore, results from several case studies and randomized trials show that gait-therapy programs incorporating end-effector type rehabilitation robots are effective $[14,16,76]$; even non-ambulatory hemiparetic subjects experienced significant improvements in gait quality after participating in gait training programs using the LokoHelp and GaitTrainer GT I $[14,16,76]$. Moreover, results from a randomized trial with the Gait Trainer GT I also revealed that the robot-based intervention outperformed conventional overground gait training [76].

Besides their large footprint and high cost, another drawback of end-effector-type rehabilitation robots is their limited capacity for balance training. The harness used to suspend the patient, artificially constrains and stabilizes trunk motion, and leads to an unnatural body weight distribution. It also inhibits the natural trunk response that the patient would exhibit - or needs to re-learn - during balance training. Additionally, all end-effector-type rehabilitation robots require the patient's feet to remain fixed to a set of actuated footplates. These devices further impede balance training because they constrain and stabilize ankle rotation and artificially enhance the patient's lateral stability. The actuated footplates used on these devices are usually designed to operate in the sagittal plane. As such, they constrain the patient's leg motion to the sagittal plane, and restrict practice of side-stepping and turning motions. In principle, end-effector type robots incorporating footplates with higher degrees of freedom can overcome some of these 
limitations. However, more complicated footplate designs would inevitably increase device cost and control complexity.

\subsubsection{Exoskeleton-type Rehabilitation Robots}

Exoskeleton-type rehabilitation robots such as the Lokomat [19], PAM-POGO [20], LOPES [21], and ALEX [23] typically consist of an actuated BWS/fall prevention system, a robotic exoskeleton, and a fully controllable treadmill. The exoskeleton mimics the structure of the human leg (to varying degrees), attaches parallel to the patient's leg, and supplements the patient's muscle-generated joint torque to help prevent buckling of the stance leg and/or to guide the swing leg through an appropriate trajectory. In general, designers of these systems are concerned with building lightweight and compliant exoskeletons [20,21], choosing an appropriate number of passive and active DOFs [20,23,77], and designing appropriate control algorithms that encourage patient involvement during gait rehabilitation therapy $[20,21,23,78]$.

The exoskeleton's compliance and degrees of freedom have a substantial impact on the constraints that the robot imposes on the user's natural walking motion $[20,51,77]$. Older versions of the Lokomat, for example, limited leg and trunk motion to the sagittal plane. A study comparing free treadmill walking with walking with the Lokomat revealed significant differences in the spatiotemporal muscle activation patterns between the two

conditions $[51,79]$. However, a similar study conducted with LOPES robot, a compliant robot with a larger number of actuated and passive DOFs, indicated a good correspondence between muscle activation patterns in free treadmill walking and treadmill walking with the LOPES exoskeleton attached. In other words, an exoskeleton without the appropriate number of active and passive DOFs will obstruct the user's natural joint and limb motions. Though there is no direct evidence available to confirm this, obstructions to natural joint motion imposed by a poorly designed exoskeleton are undesirable because they could lead to patients learning abnormal gait patterns. 
Exoskeleton-type robots which limit leg and/or trunk motion to the sagittal plane (e.g., older versions of the Lokomat) cannot be used for targeted balance training. The results of a study comparing conventional BWSTT and Lokomat walking [80] provides some evidence for this hypothesis; though all patients observed a noticeable improvement in balance, the group of patients that participated in conventional BWSTT observed nearly twice the gains in balance improvement as the group that trained with the Lokomat [80]. The limited gains in balance improvements observed with the Lokomat are likely due to the constraints it imposed on the patient's pelvis and lower extremity motions. These motion constraints enhance lateral stability [51] and reduce the active effort that patients need to exert to maintain their balance [48].

The harnesses used with some exoskeleton-type rehabilitation robots are undesirable because they constrain and stabilize trunk motion and limit the patient's ability to learn appropriate postural responses. Some robotic systems such as ALEX, LOPES, and PAM-POGO were intentionally designed to impose minimal constraints on the user's natural toros/pelvis motion, and to force patients to actively work to maintain their balance $[20,21,23]$. Thus, these particular robots could also be used for targeted balance training. However, rehabilitation platforms using exoskeleton-type robots are primarily designed to be used with treadmills. As such, these rehabilitation systems do not easily allow patients to practice CIS reactions such as side-steps, that are crucial for balance and balance training [66]. Patients can only practice such activities if these exoskeletons were used with specialized treadmills that allow unconstrained walking [81], or if the exoskeletons were attached to a mobile base that followed the user's motion. Additionally, exoskeleton-based gait rehabilitation systems are costly, have a large footprint, and are challenging to control. These three factors in particular make these systems less likely to be adopted in smaller physiotherapy clinics and hospital that do not have the appropriate resources to support their use. 


\subsubsection{Actuated Mobile Gait Trainers}

Actuated mobile gait trainers consist of an actuated mobile base, a bodyweight support mechanism fixed to the mobile base, and, in some cases, an exoskeleton or manipulator for assisting the swing leg. In contrast to the rehabilitation robots described above, these devices are specifically designed to allow patients to practice overground walking [71-73,82]. The BWS/fall prevention mechanisms incorporated into these devices also separate them from intelligent mobility aids (i.e., actuated walkers) designed to assist users in navigating through indoor environments [83-88].

The KineAssist robot developed by Kinea Design is perhaps the most sophisticated actuated mobile gait trainer that has been developed to date [71]. It possesses an omnidirectional mobile base consisting of two castors at the front, and two independently actuated and steered wheels at the rear of the base. An actuated trunk support attached to the user's chest can be used to stabilize (or perturb) the patient's trunk, and to catch a patient during a fall. A separate, actuated pelvis support mechanism is used to unload the patient's body weight and acts as a vertical fall prevention mechanism. Both the trunk and pelvis support mechanisms are designed with numerous passive and active degrees of freedom and can be controlled to provide minimal interference, or to fully constrain and stabilize the patient's torso and pelvis. The primary drawbacks of this device - though it is the gold standard in many respects - are its lack of a swing-leg assistance mechanism and its complex design which increases its cost.

The Where I and Where II robots developed by Seo and Lee [72], and the actuated mobile gait rehabilitation device developed by Lee and Lee [82] have differentially steered mobile bases that are commanded to track the user's torso velocity. The actuated BWS mechanisms on the Where I and Where II robots achieve constant body weight unloading by strictly regulating the user's center of mass position, while a model-based force control algorithm is used to control body weight unloading on the robot developed by Lee and Lee. 
All three gait trainers enable patients to walk overground in straight lines or curved paths. However, the mobile bases are not capable of omnidirectional motion, and do not allow patients to perform activities such as side-stepping. Moreover, the non-holonomic motion bases may also inhibit lateral pelvis motions and the user's lateral stability. Furthermore, the BWS systems on all three devices constrain torso motion. As a result, these devices may not be suitable for balance training. Finally, both the Where I and Where II robots regulate body weight unloading by regulating the patient's vertical center of mass position to a fixed value. However, studies suggest that BWS systems that do not accommodate vertical center of mass oscillations during gait, result in abnormal gait kinematics and kinetics $[35,38]$. Thus, the Where I and Where II robots are inferior training tools in comparison to the KineAssist.

The WalkTrainer device developed by Stauffer et. al [73] allows paraplegic patients with minimal muscle control to practice overground walking. It combines a differentially steered mobile base, an actuated harness-based BWS system, a lower extremity exoskeleton, and closed-loop muscle stimulation electronics into a single, coordinated system. The six actuated DOFs of the pelvis support mechanism allow precise control over the patient's pelvis motion, while the leg exoskeletons allow control over the patient's hip, knee, and ankle flexion. While this device is highly capable, and well suited for paraplegic patients in particular, its restrictive design may not be well-suited for higher functioning patients.

\subsubsection{Human Augmentation Exoskeletons}

A variety of stand-alone, exoskeleton-based devices such as Bleex [89], HAL-5 [90], ReWalk $^{T M}$ [91], RoboKnee [92], and the devices being developed by Berkley Bionics [93] and Raytheon and Sarcos [94] have applications to gait rehabilitation. However, these devices are primarily designed for augmenting the load bearing capabilities of human beings. Generally speaking, designers of these devices are concerned with ergonomic issues, energy efficiency, minimizing device weight, maximizing payload capacity, and 
designing control strategies that amplify user strength. These devices could also be used for gait rehabilitation and would exhibit similar advantages and disadvantages as the exoskeleton-type rehabilitation robots described above.

\subsection{The Bungee Mobility Trainer}

The Bungee Mobility Trainer (BMT) [95] is a patented mobile BWS system that is used in hospitals and clinics throughout North America. This section provides a brief overview of the device and its key features. The BMT is introduced here since it was used in the first prototype of the GaitEnable gait trainer developed for this research Chapter 4.

As shown in Figure 2.4, the device consists of a mobile base and a passive elastic mechanism that supports the patient's pelvis from below. The width of its mobile base can be adjusted to allow it to fit through standard doorways, and optional forearm supports can be attached to the device for individuals with a weak trunk and upper extremities. Clinical applications for this device include brain or spinal cord injury, stroke, multiple sclerosis, cerebral palsy, and Parkinson's disease [95].

The BMT's omnidirectional mobile base is composed of four offset castors, and BWS is provided by a Bungee cord-resisted passive mechanism. In the event that a patient stumbles or loses their balance, the device provides the patient with increased body-weight support to assist recovery. However, if the patient is unable to recover and continues to fall, the passive mechanism tends towards a final configuration that results in the user approaching a seated position. Since the BMT provides support from below and does not require the use of a harness, it enables patients to practice the protective trunk postural reactions necessary to prevent falls. Furthermore, since the device is capable of omnidirectional motion and does not restrict the user's leg motion, it can be used for balance training activities such as one leg balance. 

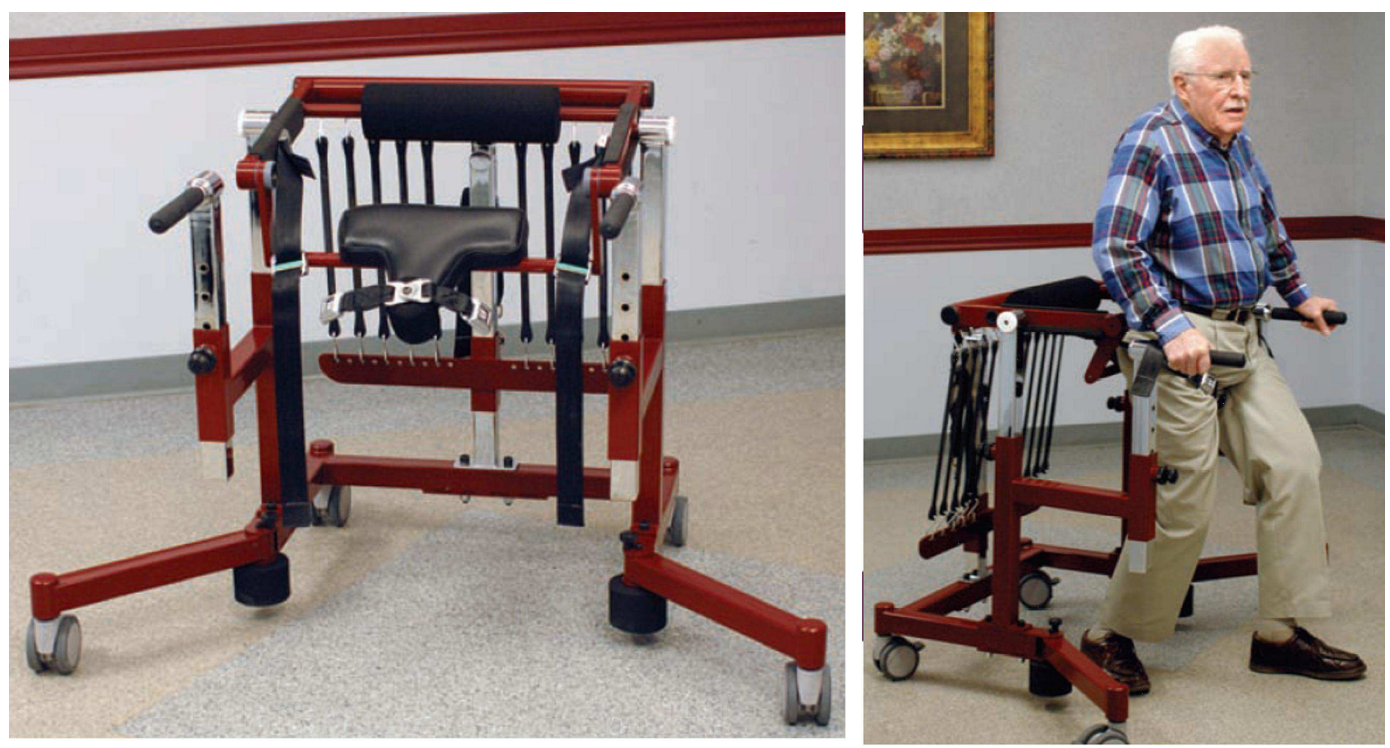

Figure 2.4: The Bungee Mobility Trainer

As shown in the simplified schematic in Figure 2.5, the BMT provides body weight unloading proportional to the extension of the bungee cords that constrain the motion of the primary hinge; the extension of the bungee cords is dependent on the patient's pelvis height, the primary hinge's vertical position, and the resting position of the bungee cords. The height adjustment mechanisms on the device allow the vertical position of the primary hinge and resting position of the bungee cords to be adjusted in increments of 1 inch. As a result, the device can easily accommodate patients of different heights. The two height adjustment mechanisms can also be manipulated to control the average amount of body weight unloading and the rate of body weight unloading that the user receives.

If the device is not configured to provide constant BWS, then support is only provided when the user's pelvis begins to fall (i.e., when the user is incapable of fully supporting their body weight and the stance leg begins to buckle). This ensures that the user is supported only when necessary, and that the non-paretic leg is not provided with unnecessary support. In contrast, the counterweight- and spring-supported overhead BWS systems used during 
BWSTT and robot-assisted BWSTT provide the same amount of support for both the paretic and non-paretic leg [96].

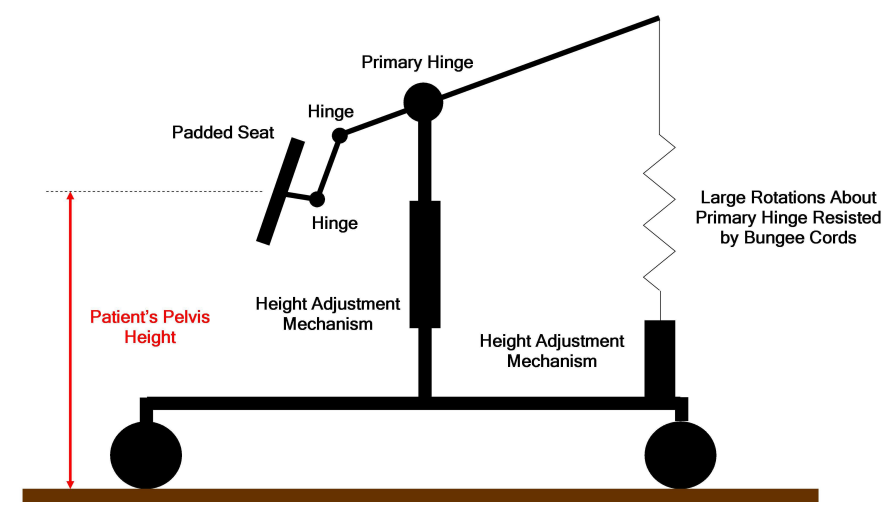

Figure 2.5: A schematic of the BMT's passive BWS mechanism

As noted in [97] and [98], pelvis motion follows a cyclical three-dimensional trajectory during treadmill walking. In particular, the vertical position of pelvis can vary on the order of several centimeters (depending on an individual's stride length [97]). The forward/backward motion of the mobile base and the rotations of the primary and secondary hinges of the passive mechanism - the ones highlighted in Figure 2.6 - accommodate pelvis height variations. Since the four castors on the mobile base allow omnidirectional motion, the patient's pelvis translations in the transverse plane also remain unconstrained (provided that the four castors are not in a singular configuration). While the device accommodates all three DOFs of pelvis translation, its mass and inertia will invariably provide some resistance to the user's pelvis motion.

The rightmost passive hinge shown in Figure 2.6 accommodates the user's trunk flexion, and the omnidirectional motion base accommodates transverse plane trunk rotations. Since there are no obstructions on the user's torso, trunk rotations about the anterior-posterior axis are completely unconstrained. The BMT also imposes minimal constraints on hip flexion, adduction/abduction and rotation if the user is properly attached to the device. 


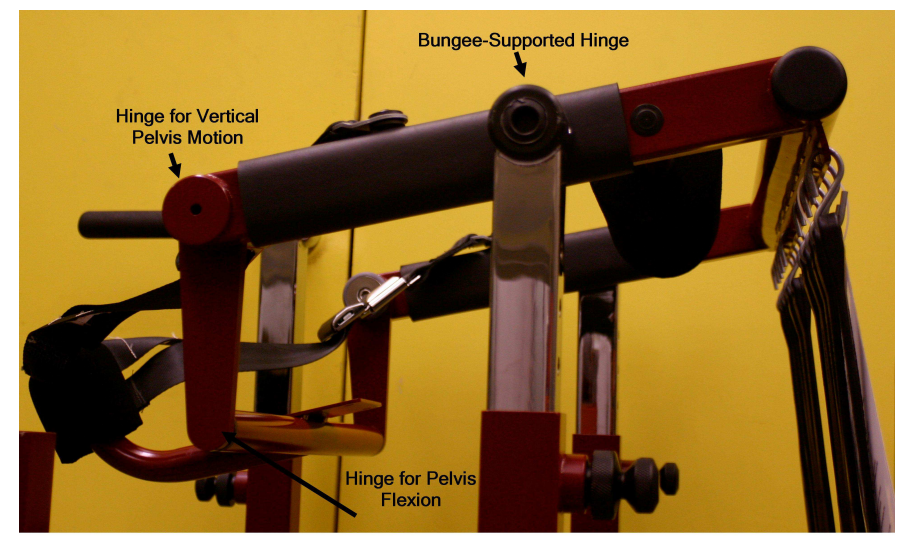

Figure 2.6: The passive linkage connecting the user's pelvis to the body weight support mechanism

However, if the belts used to secure the patient to the padded seat do not follow the contours of the patient's pelvis (and wrap around the patient's thighs instead), then the belts may limit hip adduction/abduction and flexion.

In summary, the BMT is a simple and practical mobile BWS system that provides fall protection and enables overground ambulation and balance training. It does not constrain leg and ankle motion to the sagittal plane, and is designed to impose minimal constraints on the user's torso, pelvis and hip motion (particularly in comparison to many gait rehabilitation robots). However, the BMT is a passive device that lacks many of the advantages that robotic gait rehabilitation systems can offer. In particular, the BMT still requires considerable therapist involvement and effort, cannot automatically provide the assistance necessary to initiate, sustain, and stabilize a patient's gait, and is not equipped with sensors for tracking patient progress and key gait parameters such as walking speed. 


\subsection{Facilitating Physical Human-Robot Interactions via Ad- mittance Control}

The control system that enables the user and robot to interact also requires careful design. At minimum, this control system should guarantee a stable interaction (e.g., user inputs generate smooth, consistent, and predictable device motions). The control system should also provide a simple and intuitive mechanism for the user to control the motion of the robot. This is important since many patients may suffer from cognitive or physical impairments that limit their ability to use computer interfaces or tactile interfaces such as joysticks. Also, a robot can be much more effective during rehabilitation therapy if it seamlessly accommodates and assists the user's volitional motion; studies have already shown that patients remain passive during rehabilitation therapy if a robot directly controls their limb or joint motions $[99,100]$. Accordingly, the interaction control system should enable patients to initiate and sustain their own body motion and receive assistance only when it is necessary $[24,99,100]$. Moreover, it is desirable to have the robot apply specific force cues for altering the user's motion in a meaningful way (e.g., some patients may need the robot to stabilize their lateral motions while they practice stepping motions in the sagittal plane). Thus, the control system should also provide a means for relating therapy-specific requirements into corresponding robot motion commands.

One approach to facilitating a human-robot interaction is to directly predict the user's voluntary motion, and to command a robot to assist that motion. For example, Fleischer and Hommel demonstrated that surface electromyography (sEMG) data collected from the surface of an individual's body may by combined with empirical muscle and body dynamics model for predicting and assisting the user's voluntary motion [101]. A variety of other modalities such as electroencephalography (EEG), electrooculography (EOG), camera-based head and eye motion tracking, etc. may also be used to predict a user's intended motion [102]. Generally speaking, such motion intent systems require sensors to be installed on the user's body (and/or their environment), and user- and application-specific 
calibration and data processing procedures for generating a motion prediction.

Motion prediction systems are difficult to implement for several reasons: i) there are numerous modalities to choose from, and many applications may require fusing information from several modalities together to generate an accurate prediction [102]; ii) collection of sEMG, EEG, and EOG data is not straightforward as electrode placement, skin moisture, muscle fatigue, blood circulation, cross-talk, etc., can all effect the quality of the data collected; iii) performing user- or application-specific calibration procedures may not be practical in clinical settings; iv) the data processing procedures used to predict motions (e.g., user-specific sEMG thresholds to initiate a robot's motion) may not be robust to new user inputs that exceed the range of the training data. In addition, there are equally significant challenges associated with defining how the robot should move once a motion prediction is available [101].

Impedance and admittance control provide an alternative. Both control frameworks provide mechanisms for commanding a robot's motion in response to force inputs from a user. Thus, the focus shifts from predicting the user's motion, to understanding how best to shape the robot's response to a given force input. Many of the challenges with predicting the user's intended motion are eliminated since only measurements of the robot's kinematics and the forces exchanged at the user and robot interface are required for implementing these controllers. Though easier to implement, both frameworks implicity assume that the user is capable of generating the force inputs necessary to command the robot's motion. This assumption does not appear to be limiting in practice, however, as numerous rehabilitation robots tested with patients already employ these control methodologies [103]. 


\subsubsection{Comparing Impedance and Admittance Control}

Both impedance and admittance control have been used extensively in robotics and haptics applications where tactile feedback and mechanical stimuli are used to render a virtual environment or to impose a specific interaction at a contact interface [103-107]. In addition to guaranteeing a stable user-device interaction, these control algorithms must also be capable of faithfully recreating the tactile sensations encoded within the virtual environment or interaction model. Though many different types of interactions are possible, at the least, these algorithms should allow rigid contact in the virtual environment to feel infinitely rigid, and free motion within the virtual environment to feel unhindered.

As noted in $[108,109]$, the performance advantages and disadvantages of both impedance and admittance control algorithms are complimentary. At the most basic level the two control approaches may be differentiated as follows: impedance control requires transform-

ing position inputs to force outputs, while admittance control requires transforming force inputs into position commands.

Impedance control requires that the haptic device's position/velocity in a virtual environment (simulated in software) be used to calculate the force that the robot applies to a user. It is best suited for displaying soft impedances and unhindered free motion [109]. This is crucial in applications where transparency and the reduction of the interaction forces is the primary objective (e.g., if a rehabilitation robot is required to feel light and easy to move with a small force input from the user). Another benefit of impedance control is that it does not require measurements of the contact forces generated at the user-device interface. As multi-DOF force sensors can be costly, this can be a major advantage in some applications.

Admittance control requires transforming the forces measured at the contact interface into corresponding position and velocity commands for the robot. It is intrinsically better 
suited for displaying large impedances and rigid contacts [109-111] (e.g., simulating a virtual wall that restricts the patient from moving in a particular direction). As noted in [109], the stiffening effect of the high-gain position control loop and makes it difficult for an admittance-controlled haptic device to exhibit highly compliant behavior.

The design of the device and the actuation system is the most important factor one must consider when choosing between impedance and admittance control. Impedance control requires accurate control over actuator forces. Thus, it is better suited for highly backdriveable haptic devices that have minimal intrinsic damping (e.g., devices designed with direct-drive motors), where accurate force is easier to achieve [111]. Admittance controller is more beneficial for nonlinear haptic devices that use pneumatic or hydraulic actuation, or servomotors with large gear ratios, as it is simpler to achieve accurate position control with such systems [111].

Designing a mobile gait trainer such as GaitEnable for impedance control is challenging because it requires that large, expensive direct drive motors be used to actuate the mobile base. In addition, sophisticated slip detection and traction control algorithms may be required to achieve direct control over the traction forces generated at the wheel-ground interface. This introduces a substantial sensing and computational burden as many traction control algorithms require measurements (or estimates) of tire adhesion forces, normal forces and/or wheel slip [112-114].

Implementing admittance control with a mobile gait trainer is more practical. As shown in further detail in Chapter 4, the simplest implementation, open-loop velocity control of the mobile base, can be achieved with local position or velocity controllers at each actuated wheel. Accordingly, the choice to use admittance control for GaitEnable was made early in the research. As noted, admittance control is best suited for displaying large impedances and rigid contacts. However, having a gait rehabilitation robot display a small impedance is of great benefit as it allows a large, heavy robotic device to feel light and easy to move. Designing an admittance controller that displays both small and large impedances, a crucial 
element of this research, is explored in further detail in Chapter 3.

\subsubsection{Example Implementation of an Admittance Controller}

As shown in Figure 2.7, admittance control transforms the force inputs generated at the human and robot contact interface into corresponding robot motion commands. These motion commands are generated by passing the force input through a dynamics simulation of the desired device dynamics (or virtual environment). A high-gain velocity/position controller may then be used to track the commanded device motion. If accurate tracking is achieved, the device displays the dynamics of the interaction model.

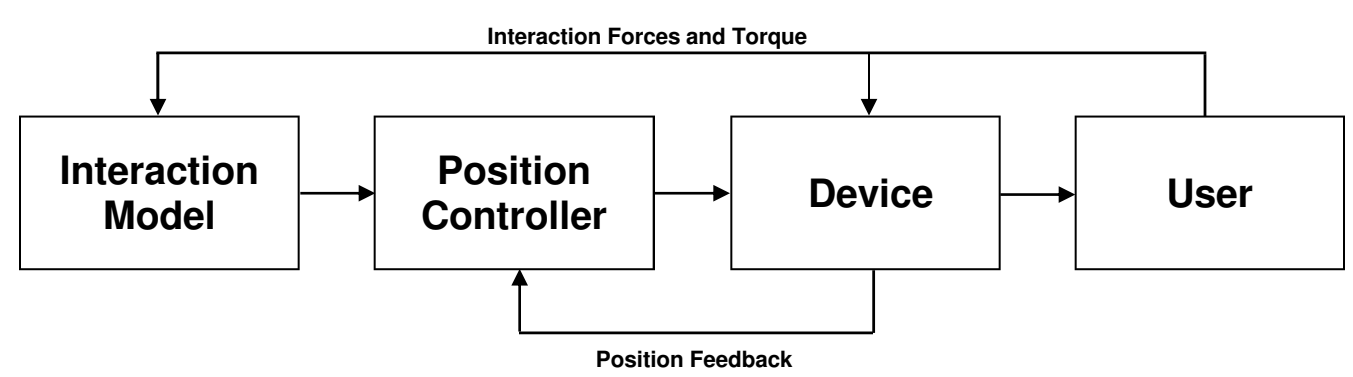

Figure 2.7: A block diagram of a generic admittance control structure.

Generally speaking, admittance control is useful in facilitating a human-robot interaction because it automatically synchronizes the human's and robot's motion, modifies the inertial properties that the robot displays to the human, and provides a systematic method for transferring forces between the robot and human. These features are illustrated in the following simple example.

Consider a robot modelled as a second-order, linear time-invariant system with dynamics: 


$$
m \ddot{x}+a \dot{x}+b x=\tau_{a c t}+\tau_{i n t},
$$

where $x$ is the position of the robot, $m, a$, and $b$ reflect the mass, damping and stiffness of the robot, respectively, $\tau_{a c t}$ is the actuator input, and $\tau_{i n t}$ in the interaction force or torque generated at the contact interface between the robot and the human (e.g., the force generated on a handle the user pushes to manipulate the robot). Admittance control allows the robot to display a desired dynamic that relates to the application of interest. For rehabilitation applications, control over the perceived mass, damping and stiffness could be valuable. Thus, an interaction model that defined the desired dynamics of the robot may be formulated as follows:

$$
m_{d} \ddot{x}_{d}+a_{d} \dot{x}_{d}+b_{d} x_{d}=\tau_{\text {int }}+\tau_{\text {virt }},
$$

where $x_{d}$ is the commanded position of the robot, $\tau_{v i r t}$ is a feedforward input that is imparted to the user at the contact interface (e.g., the user feels $\tau_{v i r t}$ applied through the handle), and $m_{d}, a_{d}$ and $b_{d}$ denote the desired mass, damping, and stiffness the user will perceive the robot to have under the admittance control. In practice, $\tau_{\text {int }}$ and $\tau_{v i r t}$ are used as inputs into (2.2) for calculating $\ddot{x}_{d}$, the acceleration the robot would exhibit if its dynamics obeyed (2.2). $\ddot{x}_{d}$ can integrated to generate the $\dot{x}_{d}$ and $x_{d}$, and any position controller may be used to track $x_{d}$. Thus, if $x=x_{d}$, then the user perceives that the robot behaves with dynamics (2.2).

From this example, we note the following:

1. The user and robot's motion are synchronized since the user simply applies a force at the contact interface in order to generate a corresponding motion from the robot. 
The need to predict the user's desired motion can be avoided, and the user is provided with a simple and intuitive mechanism for commanding the motion of the robot.

2. The design of the desired impedance, i.e., the selection of $m_{d}$, in this particular example, provides a means for modifying the inertial properties that the robot displays to the user. If $m_{d}$ is chosen to be much smaller than $m$, then the desired goal of making the robot feel lighter is achieved.

3. The addition of the feedforward input $\tau_{v i r t}$ in the desired system dynamics provides a means for generating forces cues at the user-device interface. $\tau_{v i r t}$ could be shaped in a variety of way to assist, resist, perturb, or stabilize the user's motion. This is possible because the admittance controller allows $\tau_{v i r t}$ to be applied to the user at the contact interface.

4. The accuracy and the stability of the position controller used to track $x_{d}$ determines the accuracy of the impedance display, and the stability of the interaction. However, guaranteeing stable interactions is not straightforward because a variety of factors can contribute to destabilizing the interaction.

\subsubsection{A Brief Review of Impedance and Admittance Control for Haptics}

Admittance control is advantageous, in part, because the high-gain positon/velocity loop used to track the robot's commanded motion better masks the nonlinearities in the haptic device's dynamics $[109,111]$. However, there is significant theoretical and experimental evidence to suggest that typical admittance control implementations are intrinsically more unstable when required to render very small or soft impedances $[109,111,115,116]$ (e.g., when a robotic gait trainer is commanded to display a small mass).

A variety of factors such as the human operator's and haptic device's dynamics, actuator bandwidth and saturation limits, and the position and admittance control loop parameters determine the stability of the user-device interaction [111]. Additionally, interaction stability is also influenced by factors related to the digital implementation of 
the admittance controller (e.g., sampling rate, quantization, computation delay, the use of a zero-order-hold, etc.,) $[109,111,115]$. However, if the human and haptic device dynamics are assumed to be linear, then a variety of different robust stability measures may be used to design (linear) compensators that guarantee stable user-device interactions [110,117-121]. While effective, these techniques require a priori information about the user's or device's dynamics models. Moreover, some design tools require an involved tuning procedure that may need to be repeated when there are significant changes in system parameters $[119,121]$.

The passivity formalism is also commonly used for designing stable impedance and admittance controllers for haptic simulations. These controllers - or the conditions that the passivity formalism places upon their design - ensure that the combined user-device system does not generate any energy. Two approaches to passivity-based control dominate the literature. The first class of approaches is based on the idea of selective energy dissipation triggered by a violation of the passivity condition (see [122-124] for examples). The second class of approaches consists of different techniques for selecting the parameters of the control loop or virtual environment model to satisfy the passivity condition [125, 126]. However, the passivity condition constitutes a conservative stability criterion. Thus, these controllers may provide poor performance (e.g., unnecessarily stringent restrictions on the minimum mass that the haptic device can display [119]).

Many of the controller design approaches described above assume that the haptic device is controlled using a linear position controller. As noted in $[127,128]$, such simple linear position controllers may not be robust enough to external disturbances and uncertainties due to modeling error. This fact has motivated the design of numerous different adaptive control algorithms (see $[127,129]$ and references therein for examples) that use standard Lyapunov stability arguments for designing stable interaction controllers. These approaches assume that the stability of the user-device interaction follows directly from the stability of the haptic device's position control loop. Other robust admittance control structures based on internal model control and time-delay estimation [128], variable structure control [130], 
and iterative learning control [131] have also been investigated, and similarly provide explicit guarantees on the interaction stability. Moreover, some research has also been directed towards the design of model-free position controllers for haptics that require little or no information about the robot's dynamic model $[132,133]$.

In contrast to ad-hoc implementations based on manual tuning of simple position controllers, the advantages of the different types of controllers described above may be summarized as follows: adaptive controllers can estimate unknown device dynamics, provide robustness against model parameter uncertainties, and guarantee interaction stability via the design of a stable position control loop; robust control-based approaches guarantee interaction stability in the presence of bounded uncertainties in the human, device, and environment dynamics; and, passivity-based approaches provide a conservative guarantee on the interaction stability driven primarily by energy transfer considerations.

Thus, one can conclude that numerous different approaches for addressing the instability generated by external disturbances and modeling uncertainties exist in the literature. However, no interaction controllers, to the authors' knowledge, have explicitly considered the problem of actuator saturation. As shown via experimental results in Chapter 3 , position-controlled robotic devices such as admittance-controlled haptic device are prone to saturation-related instabilities $[134,135]$. Moreover, saturation-instigated instabilities are particularly insidious and dangerous because they are not easy to predict, and highly dependent on the input the user applies to the device. Thus, the effects of actuator saturation deserve a specific consideration when a haptic device is expected to exhibit highly compliant behaviour.

The issue of designing interaction controllers for displaying soft impedances has also been considered by several researchers [119, 121, 129, 136-139]. Some controllers use a feedforward term in the control law that is proportional to the interaction force or the exogenous force generated by the human operator $[129,136]$ or vary the virtual 
damping in the reference model in accordance to the user's intention to accelerate [139]. In contrast, several other approaches are based on the idea of substituting the passivity criterion with less conservative robust stability measures during the controller design procedure $[119,121,137,138]$. Though effective, these controllers can be difficult to design or implement, and require some information about the device and operator dynamics.

To summarize, a variety of factors can contribute to an unstable human-robot interaction. One can directly consider some or all of these disparate effects (e.g., as in $[115,116,118,119])$, and derive stability boundaries for the coupled human and robot system to guide controller design. One can also resort to using more complicated controllers such as adaptive or robust controllers $[128,129,132,133]$, but without guarantee that they will work under actuator saturation, and with the requirement that some information about the user's and device's dynamics model be known ahead of time. Some passivity-based controllers are simple to implement, and do not require any information about user's or device's dynamics models $[123,124]$; however, one must accept the penalty of conservative performance in return. Accordingly, this research investigated the design and experimental evaluation of a novel interaction control methodology that is minimal and model-free, but capable guaranteeing robust stability and improved performance. 


\section{Chapter 3}

\section{Stable Interactions in Admittance-controlled Haptic Devices}

In an admittance-controlled haptic device, the force the user applies to a robot is transformed into the robot's motion command. As the user pushes on the device, the motion of the robot influences the motion of the user, and the motion of the user, in turn, influences the motion of the robot. As demonstrated in Chapter 2, the complex interaction that ensues between the user and the device can be harnessed to achieve different goals (e.g., to obscure the inertial properties of the robot, or to limit or augment the user's strength, etc.). However, it is also prone to instabilities such as erratic and unpredictable motions of the coupled human-robot system and oscillations. This chapter focuses on the stability problem, and introduces two novel mechanisms - hereafter referred to as the impedance manipulations - that ensure stable human-robot interactions.

Section 3.1 illustrates two specific task-space and joint-space admittance control structures to help motivate the description the impedance manipulations. The first manipulation - dynamically-varying limits imposed on the haptic device's commanded acceleration - is described in Section 3.2. A Lyapunov stability analysis confirms that the acceleration limits ensure the stability of haptic device's position control loop. Section 3.3 presents the second manipulation - error feedback in the haptic device's commanded acceleration - and discusses how it helps stabilize the interaction. Experiments carried out 
to demonstrate the effectiveness of the impedance manipulations are presented in Section 3.4. The chapter concludes with a discussion about how the impedance manipulations can be used in generic position control problems.

\subsection{Admittance Control of Haptic Devices}

Figure 3.1 presents a block diagram of the enhanced admittance controller proposed in this chapter. A comparison of Figures 2.7 and 3.1 reveals that the main difference between a typical admittance controller and the enhanced controller is the addition of the error feedback and acceleration limits in between the interaction model and position controller. This section describes how the admittance control can be formulated for a generic manipulator in both task- and joint-space, and provides additional details about suitable interaction models, and position controllers.

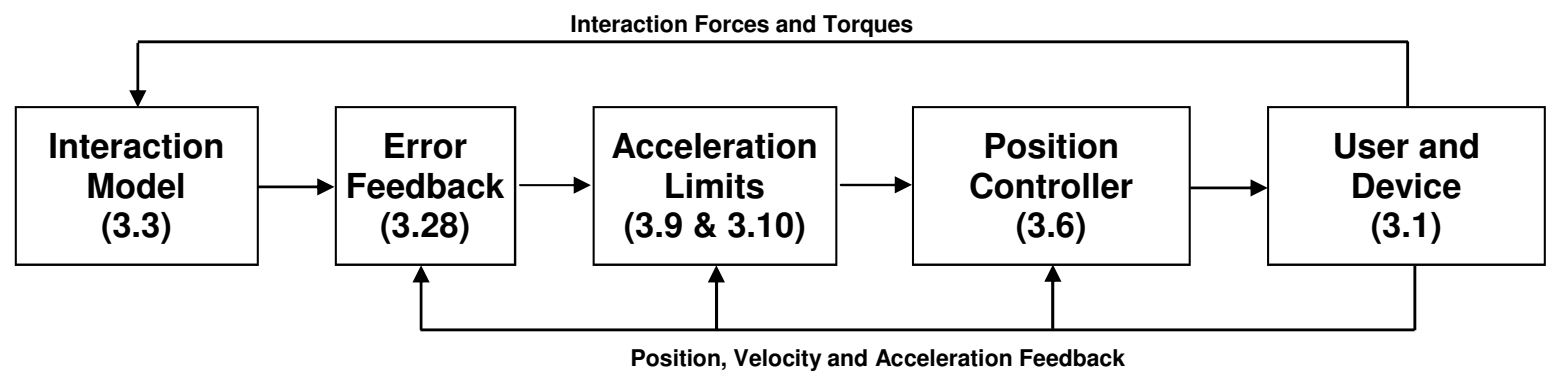

Figure 3.1: A block diagram of the enhanced admittance controller. Bracketed numbers indicate corresponding equation numbers.

\subsubsection{Haptic Device Dynamics}

The joint-space dynamics of an $n$ degree of freedom haptic device that contacts a user at a single point, may be expressed as

$$
M(q) \ddot{q}+N(q, \dot{q})=J(q)^{T} F_{\text {int }}+\tau,
$$


where $q \in R^{n}$ is a set of $n$ generalized coordinates used to parameterize the configuration of the system, $M(q) \in R^{n \times n}$ denotes the positive-definite inertia matrix, $N(q, \dot{q})$ is the collection of the Coriolis, damping, and gravitational terms, $F_{\text {int }}$ is the interaction force measured at the user-device contact interface, $J(q)$ is the Jacobian matrix, and $\tau$ is the vector of actuator torques.

Assuming that the Jacobian matrix is non-singular, the dynamics in task space may be written as,

$$
M_{x} \ddot{x}+N_{x}(x, \dot{x})=F_{\text {int }}+u
$$

where $u=J^{-T}(q) \tau, \quad N_{x}=J^{-T}(q)\left(N(q, \dot{q})-M(q) J^{-1}(q) \dot{J}(q, \dot{q}) J^{-1}(q) \dot{x}\right), \quad$ and $M_{x}=J^{-T}(q) M(q) J^{-1}(q)$. Many robust and model-free position controllers guarantee stability subject to bounds on the inertia, Coriolis, and gravity terms $[128,132,133]$. Knowledge of these bounds is not required for implementing the impedance manipulations.

\subsubsection{Target Impedance and Reference Trajectory Generation}

The desired dynamics at the contact interface may be defined using any reference model that characterizes the haptic interaction of interest. If one wishes to modify the mass, stiffness, and damping properties of the robot in joint-space, the following joint-space reference impedance model may be used:

$$
M_{d} \ddot{q}_{r}+C_{d} \dot{q}_{r}+K_{d} q_{r}=J(q)^{T}\left(F_{i n t}+F_{a}\right)
$$


where $q_{r}$ is the reference trajectory the robot must follow to display the desired interaction, $M_{d}, C_{d}$, and $K_{d}$ define the target inertia, damping, and stiffness of the haptic device, and $F_{a}$ is the feedforward force to be applied to the human operator through the contact interface. The haptic device displays the target impedance if its position controller allows accurate tracking of the reference trajectory $q_{r}$. Moreover, if the device's joints rotate with the user's joints, $M_{d}, C_{d}$, and $K_{d}$ manipulate the perceived inertia, damping, and stiffness of the user's joints $[140,141]$.

If the admittance controller is defined in the task-space, then the following reference model may be used to alter the end-effector's apparent inertia, damping, and stiffness:

$$
M_{d, x} \ddot{x}_{r}+C_{d, x} \dot{x}_{r}+K_{d, x} x_{r}=F_{\text {int }}+F_{a},
$$

where $x_{r}$ is the task-space reference trajectory the robot must follow to display the reference dynamics, and $M_{d, x}, C_{d, x}$, and $K_{d, x}$ define the target impedance of the haptic device's end-effector.

\subsubsection{Joint Space PD Control Formulation}

As shown in Figure 3.1, a position controller must be used track the desired trajectory that results after the impedance manipulations modify the output of the interaction model. While any position controller could be used, using a proportional $(\mathrm{P})$, or proportional derivative $(\mathrm{PD})$ controller is advantageous as it results in a model-free position control that is simple to implement. 
Using $q_{r}$ as the position command, the filtered tracking error for the $i^{\text {th }}$ controlled joint, $s_{i}$, may be defined as

$$
\begin{aligned}
& s_{i}=\left(\dot{q}_{r, i}-\dot{q}_{i}\right)+\phi\left(q_{r, i}-q_{i}\right), \\
& s_{i}=\dot{e}_{i}+\phi e_{i},
\end{aligned}
$$

where $\phi$ is a positive constant, $e$ denotes a vector of joint-space tracking errors, and the subscript $i$ denotes the $i^{t h}$ component of a vector quantity. Accordingly, the PD control law at the $i^{\text {th }}$ controlled joint is defined as,

$$
\tau_{i}=K_{i} s_{i}
$$

where $K_{i}$ is a positive constant. $K_{i} \phi$ and $K_{i}$ correspond, respectively, to the proportional and derivative gains of the PD controller used at the $i^{\text {th }}$ joint.

\subsubsection{Task Space PD Control Formulation}

For a task-space admittance control formulation, the filtered tracking error along the $i^{\text {th }}$ coordinate direction may be defined as,

$$
\begin{aligned}
s_{x, i} & =\left(\dot{x}_{r, i}-\dot{x}_{i}\right)+\phi_{x}\left(x_{r, i}-x_{i}\right), \\
s_{x, i} & =\dot{e}_{x, i}+\phi_{x} e_{x, i} .
\end{aligned}
$$


where $\phi_{x}$ is a positive constant, and $e_{x}$ denotes the vector of task-space tracking errors. The task-space PD control law may then be written as,

$$
u_{i}=K_{x, i} s_{i}
$$

where $K_{x, i}$ is a positive constant. $K_{x, i} \phi_{x}$ and $K_{x, i}$ correspond, respectively, to the proportional and derivative gains of the $\mathrm{PD}$ controller used along the $i^{\text {th }}$ coordinate direction. The actuator joint torques $\tau_{i}$ - the inputs that must be manipulated to control the motion of the device - are related to the task space actuation forces $u_{i}$ via the relation $\tau=J^{T} u$. Formulating the task space PD controller in this manner avoids the inversion of the mass matrix $M_{x}$.

\subsection{Position Control Loop Stability}

Figure 3.1 shows that the impedance manipulations act in between the reference model and the position controller. This allows them to shape the output of the reference model, $\ddot{q}_{r}$ or $\ddot{x}_{r}$, as necessary, to ensure a stable, oscillation-free user-device interaction. This section focuses on the acceleration limits block, and presents a novel method for restricting the maximum and minimum allowable values of $\ddot{q}_{r}$ (or $\ddot{x}_{r}$ ) that ensure that $e$ (or $e_{x}$ ) are bounded.

\subsubsection{Problem Statement}

The acceleration limits presented below exploit the notion of uniform ultimate boundedness (UUB) [142] as a means of ensuring a stable user-device interaction. Stability in the sense

of UUB implies that $\left\|s\left(t_{o}\right)\right\| \leq l \Rightarrow\|s(t)\| \leq m \quad \forall t>t_{o}+T(l, m)$, for some positive constants $l$ and $m$, and function $T=T(l, m)$. The acceleration limits described in the theorems below ensure that the filtered tracking errors $s$ and $s_{x}$ are UUB. This results in 
$\|e\|$ and $\left\|e_{x}\right\|$ being bounded.

Controller design using robust $[110,118,130]$ and adaptive control $[127,129]$ principles requires shaping the control signal or selecting the controller gains, to ensure the stability of the position control loop. In contrast, the impedance manipulations intermittently alter the target impedance to stabilize the position control loop. Though a PD control law was proposed in Section 3.1, any other position control law (e.g., P, PID, sliding-mode, or computed torque controllers) may be used in conjunction with the manipulations.

\subsubsection{Guaranteeing the Stability of the Joint-Space PD Controller}

Appropriately designed limits on the maximum allowable reference acceleration $\ddot{q}_{r}$ can ensure that the joint-space filtered tracking error $s_{i}$ is UUB. An example of one possible pair of such limits is presented in the theorem below.

Theorem 1. Consider a haptic device with dynamics (3.1) that is required to display an arbitrary, second-order target impedance. Independent of the position controller used, constraining the maximum and minimum allowable value of $\ddot{q}_{r, i}$ using the following dynamic reference acceleration limits,

$$
\ddot{q}_{r, i}>-\phi \dot{e}_{i}-\psi \operatorname{sgn}\left(s_{i}\right)-\gamma s_{i}-\eta
$$

$$
\ddot{q}_{r, i}<-\phi \dot{e}_{i}-\psi \operatorname{sgn}\left(s_{i}\right)-\gamma s_{i}+\eta
$$


where $\gamma, \psi$, and $\eta$ are positive constants, and $\operatorname{sgn}(\cdot)$ denotes the signum function, ensures that the joint-space tracking error along the $i^{\text {th }}$ coordinate direction $e_{i}$ is UUB with the following bound:

$$
\left|e_{i}\right| \leq \frac{\eta}{\gamma \phi}
$$

Proof. Consider the candidate Lyapunov function

$$
V=\frac{1}{2} s_{i}^{2}
$$

Using (3.5), the time derivative of (3.12) evaluates to

$$
\begin{aligned}
\dot{V} & =s_{i} \dot{s}_{i} \\
& =s_{i}\left(\ddot{q}_{r, i}-\ddot{q}_{i}+\phi \dot{e}_{i}\right) .
\end{aligned}
$$

When the reference acceleration is less than the minimum allowable value defined in (3.9), and the minimum allowable value is substituted into (3.13), the time derivative of the candidate Lyapunov function approaches the following extremum:

$$
-\psi \operatorname{sgn}\left(s_{i}\right) s_{i}-\gamma s_{i}^{2}-\eta_{i} s_{i}-\ddot{q}_{i} s_{i}
$$


Similarly, when the reference acceleration exceeds the upper limit defined in (3.10), and the upper limit is substituted into (3.13), the time derivative of the candidate Lyapunov function reaches the following extremum:

$$
-\psi \operatorname{sgn}\left(s_{i}\right) s_{i}-\gamma s_{i}^{2}+\eta_{i} s_{i}-\ddot{q}_{i} s_{i}
$$

It follows from (3.14) and (3.15) that an exact expression for $\dot{V}$ may be defined as follows:

$$
\dot{V}=-\psi \operatorname{sgn}\left(s_{i}\right) s_{i}-\gamma s_{i}^{2}+\alpha\left(\ddot{q}_{r, i}\right) \eta_{i} s_{i}-\ddot{q}_{i} s_{i} .
$$

where $-1 \leq \alpha\left(\ddot{q}_{r, i}\right) \leq 1$ is a function that depends on the value of the reference acceleration $\ddot{q}_{r}$. $\alpha$ varies linearly between 1 when $\ddot{q}_{r}$ is at its maximum allowable value in (3.10), to -1, when $\ddot{q}_{r}$ is at its minimum allowable value in (3.9). In the absence of acceleration feedback, $\psi \operatorname{sgn}\left(s_{i}\right)$ in $(3.16)$ compensates for the effects of the unmeasured joint acceleration. If the maximum acceleration that can be generated by the joint is denoted by $\ddot{q}_{i, \max }$, and $\psi$ is selected such that $\psi \approx \ddot{q}_{i, \max }$, then the worst-case approximation of (3.16) (i.e., when $\left.\left|\ddot{q}_{i}\right|=\ddot{q}_{i, \max }\right)$ yields the following expression:

$$
\dot{V}=-\gamma s_{i}^{2}+\alpha\left(\ddot{q}_{r, i}\right) \eta_{i} s_{i}
$$

It follows from (3.17) that $\dot{V}<0$ whenever $\left|s_{i}\right|>\frac{\eta|\alpha|}{\gamma}$. As $|\alpha| \leq 1$ by definition, considering the most critical scenario when $|\alpha|=1$, yields a conservative error convergence condition that $\dot{V}<0$ whenever $\left|s_{i}\right|>\frac{\eta}{\gamma}$. Next, let $s_{o, i}$ denote the initial value of filtered tracking error $s_{i}$. If $\left|s_{o, i}\right|>\frac{\eta}{\gamma}$, then $\dot{V} \leq 0$, and $V$ must decrease monotonically until $\left|s_{i}\right| \leq \frac{\eta}{\gamma}$. From that time on, $\left|s_{i}\right| \leq \frac{\eta}{\gamma}$ since $\dot{V}=0$ on the boundary $\left|s_{i}\right|=\frac{\eta}{\gamma}$. This is sufficient to show that 
$s_{i}$ is UUB with ultimate bound $\left|s_{i}\right| \leq \frac{\eta}{\gamma}$.

As (3.5) defines a first-order filter for $s_{i}$, the tracking error $e_{i}$ may be viewed as the output of this filter. Given that first-order systems do not exhibit overshoot, and that $\left|s_{i}\right| \leq \frac{\eta}{\gamma}$, the steady state response of (3.5) to a step change of $\frac{\eta}{\gamma}$ approximates the bound on the maximum position tracking error. This evaluation yields the following bound:

$$
\left|e_{i}\right| \leq \frac{\eta}{\gamma \phi}
$$

Remark 1. The values of $\phi, \gamma$, and $\eta$ control the maximum tracking error of the position control loop. In practice, $\eta$ should be selected to be significantly larger than $\psi$, and $\phi$ and $\gamma$ can subsequently be chosen to achieve a desired tracking error specification. Increasing $\phi$ or $\gamma$ (or reducing $\eta$ ) limits dynamic motions. Thus, iterative tuning may be required to achieve the desired trade-off between minimizing tracking error and allowing large haptic device accelerations.

Remark 2. Only measurements of $q$ and $\dot{q}$ are required to implement the acceleration limits. Accurate dynamics models of the user and device are not required. If simple position controllers are used at each joint, then large control gains may be used to compensate for coupling effects and the uncompensated dynamics [109]. Using large control gains is possible in practice since the acceleration limits, and the second impedance manipulation described in Section 3.3, act as a safety net that preserve the stability of the position control loop.

Remark 3. The acceleration limits can be applied independently at each of the $i$ joints of the robot to ensure that $\left|q_{r}-q\right|$ is bounded. The size of this bound would depend on the 
values of $\phi, \gamma$, and $\eta$ used at each joint.

Remark 4. Theorem 1 only requires that the haptic device's acceleration be bounded. This minimum restriction ensures that the time derivative of the Lyapunov function (3.13) remains bounded for all possible actuator inputs. As no real haptic device can exhibit unbounded accelerations, the acceleration limits may be used with any type of haptic device.

Remark 5. Stable interactions cannot be guaranteed in situations where the reference model forces an unstable interaction (e.g., if excessive damping compensation is applied via a negative-definite damping matrix $B_{d}$ in (3.3)). In this scenario, the results of Theorem 1 would still hold, and the tracking error would remain bounded, but the haptic device may still observe unsafe or erratic motions.

Remark 6. Equations (3.9) and (3.10) limit the magnitude of the position tracking error by limiting the maximum allowable reference acceleration. Hence, the haptic device will not display the target impedance while (3.9) or (3.10) are active. However, the limits will not interfere with user's ability to manipulate the device. The device will simply feel heavier or less responsive for the duration of time that the limits are active.

\subsubsection{Guaranteeing the Stability of the Task-Space PD Controller}

The following theorem demonstrates that appropriately designed limits on the task-space acceleration $\ddot{x}_{r}$ can ensure the stability of the task-space filtered tracking error $s_{x}$.

Theorem 2. Consider a haptic device with dynamics (3.2) that is required to display an arbitrary, second-order target impedance. Independent of the position controller used, constraining the maximum and minimum allowable value of $\ddot{x}_{r, i}$ using the following dynamic reference acceleration limits, 


$$
\ddot{x}_{r, i}>-\phi_{x} \dot{e}_{x}-\psi_{x} \operatorname{sgn}\left(s_{x, i}\right)-\gamma_{x} s_{x, i}-\eta_{x},
$$

$$
\ddot{x}_{r, i}<-\phi_{x} \dot{e}_{x}-\psi_{x} \operatorname{sgn}\left(s_{x, i}\right)-\gamma_{x} s_{x, i}+\eta_{x},
$$

where $\gamma_{x}$, and $\psi_{x}$, and $\eta_{x}$ are positive constants, ensures that the task-space tracking error along the $i^{\text {th }}$ coordinate direction $e_{x, i}$ is UUB with the following bound:

$$
\left|e_{x, i}\right| \leq \frac{\eta_{x}}{\gamma_{x} \phi_{x}}
$$

Proof. Consider the candidate Lyapunov function

$$
V_{x}=\frac{1}{2} s_{x, i}^{2}
$$

Using (3.7), the time derivative of (3.22) evaluates to

$$
\begin{aligned}
\dot{V}_{x} & =s_{x, i} \dot{s}_{x, i} \\
& =s_{x, i}\left(\ddot{x}_{r, i}-\ddot{x}_{i}+\phi_{x} \dot{e}_{x}\right) .
\end{aligned}
$$

When the reference acceleration is less than the minimum allowable value defined in (3.19), and the minimum allowable value is substituted into (3.23), the time derivative of the candidate Lyapunov function approaches the following extremum: 


$$
s_{x, i}\left(-\psi_{x} \operatorname{sgn}\left(s_{x, i}\right)-\gamma_{x} s_{x, i}-\eta_{x}-\ddot{x}_{i}\right) .
$$

Similarly, when the reference acceleration is greater than the maximum allowable value defined in (3.20), and the maximum allowable value is substituted into (3.23), the time derivative of the candidate Lyapunov function approaches the following extremum:

$$
s_{x, i}\left(-\psi_{x} \operatorname{sgn}\left(s_{x, i}\right)-\gamma_{x} s_{x, i}+\eta_{x}-\ddot{x}_{i}\right) .
$$

If the absolute value of the maximum acceleration is denoted as $\ddot{x}_{i, \max }$, then arguments similar to those in the proof of Theorem 1 may be used to show that $s_{x, i}$ is UUB with bound:

$$
\left|s_{x, i}\right| \leq \frac{\eta_{x}}{\gamma_{x}}
$$

As (3.7) defines a first-order filter for $s_{x, i}$, the task-space tracking error $e_{x, i}$ may be viewed as the output of this filter. Given that first-order systems do not exhibit overshoot, and that $\left|s_{x, i}\right| \leq \frac{\eta_{x}}{\gamma_{x}}$, the steady state response of (3.7) to a step change of $\frac{\eta_{x}}{\gamma_{x}}$ approximates the bound on the maximum task-space position tracking error. This evaluation yields the following bound:

$$
\left|e_{x, i}\right| \leq \frac{\eta_{x}}{\gamma_{x} \phi_{x}} .
$$


Remark 1. The task space acceleration limits (3.19) and (3.20) stabilize the position control loop, and the values of $\phi_{x}, \gamma_{x}$, and $\eta_{x}$ control the bound on the task-space tracking error $e_{x, i}$. As with the joint-space acceleration limits, $\eta_{x}$ should be selected to be significantly larger than $\psi_{x} . \phi_{x}$ and $\gamma_{x}$ can then be tuned to achieve the desired tracking error specification.

Remark 2. The task-space acceleration limits can be applied independently along each coordinate direction to ensure that $e_{x}$ is UUB. The size of this bound would depend on the values of $\phi_{x}, \gamma_{x}$, and $\eta_{x}$ used for each of the $i$ coordinate directions.

\subsection{Oscillation Attenuation at Low Apparent Inertia Settings}

Typical admittance control formulations approach a fundamental stability limit when a haptic device is commanded to display a small, pure mass $[109,111,115,116]$. It is difficult to pinpoint a specific cause for this instability, as it arises from the coupling of the user, device, reference model, and position controller dynamics. If one uses linear models to approximate all these dynamics, then it can be shown that a variety of parameters such as the device mass [116], sampling rate [115,116], controller structure and gains $[115,119]$, and the damping coefficient in the reference model [115] can all influence the minimum mass that a haptic device can safely display.

The reference acceleration limits proposed in Section 3.2 were purposely designed without consideration of the specifics of the user, device, and interaction model dynamics. Thus, the position control loop of the device should remain stable and generate bounded errors when the user and device are coupled, irrespective of the mass specified in the interaction model. Experiments performed with 3 different haptic devices confirmed this. However, it was observed that all the haptic devices tested exhibited sustained oscillations when commanded to display a small mass or inertia. Figure 3.2, which presents a phase 
portrait of the filtered error dynamics, shows an example of this behaviour. The legend in the figure denotes the inertia reductions tested. Inertia reductions close to $100 \%$ denote a desired inertia close to zero. In fact, the desired inertia tested at a $99.995 \%$ reduction corresponded to $0.000005 \mathrm{kgm}^{2}$.

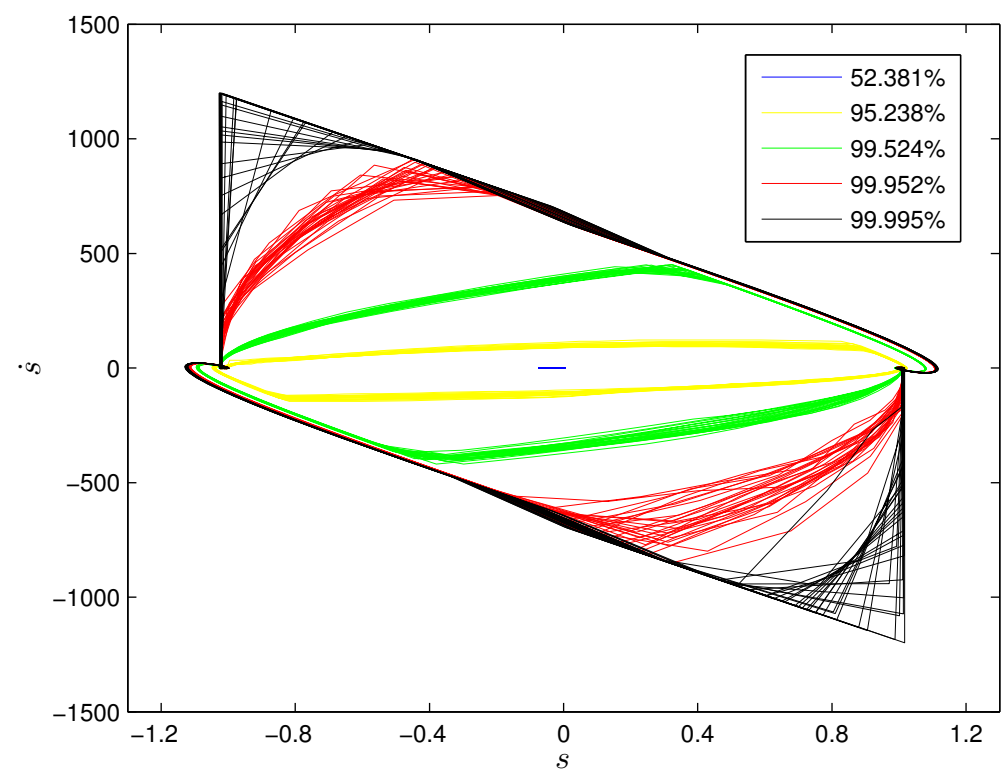

Figure 3.2: Experimental results from a 1-DOF haptic device displaying the emergence of limit cycles at consecutively lower inertia reductions.

The results in this figure demonstrate that limit cycles, i.e., sustained oscillations, emerged at an inertia reduction of $95.238 \%$. The size of the limits grew in magnitude as successively larger inertia reductions were tested. It should be noted that the user was simply attached to the device and remained passive as the haptic device shook in place. Thus, these limit cycles represent a characteristic behaviour that emerges as a result of the coupled human-robot system interacting with the acceleration limits. The figure also demonstrates that limit cycles are stable, and that $s$ is UUB with bound $|s| \leq \frac{\eta}{\gamma} \leq 1.2$, exactly as Theorem 1 states. However, these sustained oscillations are undesirable because they could damage the device or harm the user. At the least, they interfere with the 
user's ability to manipulate the device. Accordingly, the second impedance manipulation proposed below, which requires reshaping $\ddot{q}_{r}$ (or $\ddot{x}_{r}$ ) with error feedback, was devised with the motivation of decreasing the size of these limit cycles. Experimental observations suggested that when the size of the limit cycle was sufficiently reduced, the minimal oscillations that remained were imperceptible to users, and users could safely and easily manipulate the haptic device. Experimental results in Sections 3.4.5 and 3.4.7 below provide direct examples of this.

\subsubsection{Error Feedback in the Target Impedance}

The stability of the human-robot interaction is reflected, in part, by the sign and magnitude of the time derivative of the Lyapunov function (3.13). A large, positive value of $\dot{V}$ implies that the tracking error is diverging quickly, and that the interaction is exhibiting a higher degree of instability. The degree of instability can be reduced by minimizing the maximum positive magnitude of (3.13). This can be achieved by feeding back $\dot{s}$ in the reference model as follows:

$$
\ddot{q}_{t, i}= \begin{cases}\ddot{q}_{r, i}+b \dot{s}_{i}, & \text { if } \dot{V} \geq 0 \\ \ddot{q}_{r, i}, & \text { if } \dot{V}<0\end{cases}
$$

where $\ddot{q}_{t}$ is the reshaped reference acceleration used to generate the haptic device's reference trajectory. Thus, $\dot{q}_{r}$ and $q_{r}$, the trajectory the position controller must track, are realized by integrating $\ddot{q}_{t}$ instead of $\ddot{q}_{r}$. The parameter $b$ is a positive constant that determines the magnitude of the stabilizing feedback used along the $i^{\text {th }}$ coordinate direction. The value of $b$ can be varied after the acceleration limit and impedance model parameters have been selected. Like the acceleration limits, (3.28) limits rapid haptic device accelerations. Thus, $b$ may be tuned to achieve the desired trade-off between attenuating oscillations and allowing large haptic device accelerations. As (3.28) requires acceleration feedback, 
additional filtering may be necessary if $\ddot{q}_{i}$ in $\dot{s}_{i}$ is estimated by double differentiation of the robot position $q_{i}$.

\section{Mechanism of Action}

To understand the effect of the error feedback, consider again the Lyapunov function,

$$
V=\frac{1}{2} s_{i}^{2}
$$

When $\dot{V} \geq 0$ and (3.28) re-shapes the commanded acceleration as $\ddot{q}_{t, i}=\ddot{q}_{r, i}+b \dot{s}_{i}$, limiting $\ddot{q}_{t, i}$ between the acceleration limits (3.9) and (3.10) to take advantage of Theorem 1 implies that:

$$
\begin{aligned}
\ddot{q}_{r, i}+b \dot{s}_{i} & <-\phi \dot{e}_{i}-\psi \operatorname{sgn}\left(s_{i}\right)-\gamma s_{i}+\eta \\
\ddot{q}_{r, i} & <-\phi \dot{e}_{i}-\psi \operatorname{sgn}\left(s_{i}\right)-\gamma s_{i}+\eta-b \dot{s}_{i}
\end{aligned}
$$

and,

$$
\begin{aligned}
\ddot{q}_{r, i}+b \dot{s}_{i} & >-\phi \dot{e}_{i}-\psi \operatorname{sgn}\left(s_{i}\right)-\gamma s_{i}-\eta \\
\ddot{q}_{r, i} & >-\phi \dot{e}_{i}-\psi \operatorname{sgn}\left(s_{i}\right)-\gamma s_{i}-\eta-b \dot{s}_{i} .
\end{aligned}
$$

Noting that $b s_{i} \dot{s}_{i}=b \dot{V}$, substituting the limit on $\ddot{q}_{r, i}$ from (3.30) into (3.13) shows that

$$
\begin{aligned}
\dot{V} & =-s_{i}\left(b \dot{s}_{i}\right)+s_{i}\left(-\psi \operatorname{sgn}\left(s_{i}\right)-\gamma s_{i}+\eta_{i}-\ddot{q}_{i}\right) \\
& =\frac{s_{i}\left(-\psi \operatorname{sgn}\left(s_{i}\right)-\gamma s_{i}+\eta_{i}-\ddot{q}_{i}\right)}{1+b} .
\end{aligned}
$$


Similarly, substituting the limit on $\ddot{q}_{r, i}$ from (3.31) into (3.13) shows that

$$
\begin{aligned}
\dot{V} & =-s_{i}\left(b \dot{s}_{i}\right)+s_{i}\left(-\psi \operatorname{sgn}\left(s_{i}\right)-\gamma s_{i}-\eta_{i}-\ddot{q}_{i}\right) \\
& =\frac{s_{i}\left(-\psi \operatorname{sgn}\left(s_{i}\right)-\gamma s_{i}-\eta_{i}-\ddot{q}_{i}\right)}{1+b}
\end{aligned}
$$

Thus, (3.32) and (3.33) show that augmenting the reference acceleration with error

feedback scales $\dot{V}$ by a factor of $(1+$ b). In other words, (3.28) helps stabilize the interaction by inhibiting rapid increases in tracking error. It is important to note that Theorem 1 holds as long as the reshaped reference acceleration $\ddot{q}_{t, i}$ is limited by the acceleration limits (3.9) and (3.10). The exposition above was only provided to illustrate how the error feedback helps stabilize the interaction.

If (3.28) is used, then the dynamics of filtered tracking error $s_{i}$ constitute a switched system, with $\dot{V}$ acting as the switching signal. The stability of the error dynamics under arbitrary switching can be guaranteed since a common Lypaunov function (3.12) was used to show that $s_{i}$ is UUB [143].

\subsubsection{Extension to Task-space Admittance Control}

An analogous expression for reshaping the task-space reference acceleration may be defined as

$$
\ddot{x}_{t, i}= \begin{cases}\ddot{x}_{r, i}+b_{x} \dot{s}_{x, i}, & \text { if } \dot{V}_{x} \geq 0 \\ \ddot{x}_{r, i}, & \text { if } \dot{V}_{x}<0\end{cases}
$$


where $\ddot{x}_{t, i}$ is the modified reference acceleration used to generate the haptic device's reference trajectory (i.e., $\dot{x}_{r, i}$ and $x_{r, i}$ are realized by integrating $\ddot{x}_{t, i}$ instead of $\ddot{x}_{r, i}$ ), and $b_{x}$ is a positive constant that determines the magnitude of the stabilizing feedback used along the $i^{\text {th }}$ coordinate direction. Considering the Lyapunov function $V_{x}$ in (3.23), and using arguments similar to those in Section 3.3.1, it can be to shown that (3.34) similarly attenuates the maximum positive magnitude of $\dot{V}_{x}$.

\subsection{Experimental Results with a 1-DOF Haptic Device}

This section describes results from a series of experiments that illustrate the benefits of using the reference acceleration limits and re-shaping the reference acceleration with error feedback. These experiments only consider a joint-space implementation of the admittance control, and were performed with the 1-DOF haptic device shown in Figure 3.3. Additional details about the experimental apparatus, procedures and the results follow.

Coupling effects, singularities, and redundancy resolution introduce additional challenges not considered in the experiments discussed below. While these experiments consider a relatively simple haptic device, Chapters 4 and 5 present experimental results with the GaitEnable gait trainer, a 3 DOF nonlinear system which exhibits coupling effects.

Only healthy participants were recruited to participate in the experiments. The apparatus and the subject recruitment, screening, and informed consent procedures were all reviewed by and received ethics clearance through the Carleton University Research Ethics Committee. 


\subsubsection{Experimental Apparatus}

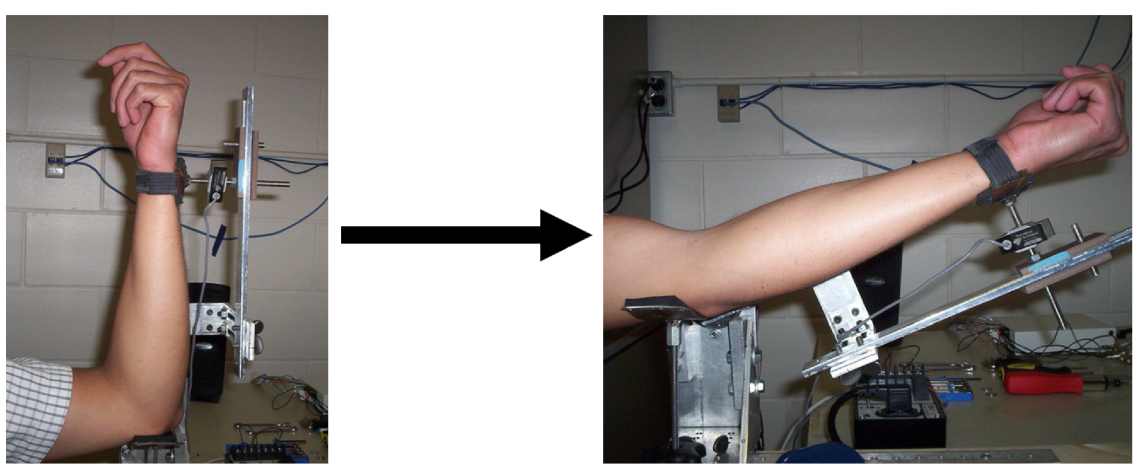

Figure 3.3: A 1-DOF haptic device for assisting elbow motions.

The experimental apparatus consists of a haptic device that can be attached to an individual's forearm. One end of the device is fixed to a stationary base. The other end of the device attaches to the user's wrist as shown in Figure 3.3. The device only allows for motor-assisted (or resisted) flexion and extension. Rotations about any other axis are limited to the mechanical joint tolerances in the system, or due to deformation of the tissue surrounding the user-device interface.

Assistance (or resistance) is provided via a servomotor comprised of a Maxon EC Powermax 200 DC brushless motor attached to a 565:1 planetary gear transmission. The servomotor can provide a continuous torque of $40 \mathrm{Nm}$ at $30 \mathrm{RPM}$. The angular position of the device arm is measured using an encoder mounted on the motor shaft. A Transducer Techniques MLP 75 force sensor mounted within the wrist connection mechanism is used to measure the user-device interaction force. Interaction torque is calculated as the product of the interaction force and the moment arm the wrist connection mechanism generates with respect to the motor's axis of rotation.

An NI PCI 6259 data acquisition card is used to interface the sensors and actuator to the control computer. The entire system is controlled using a PC-based real-time 
control system implemented using Matlab's xPC Target toolbox. Both the position and admittance control loops operate at a sampling rate of $2000 \mathrm{~Hz}$.

This experimental apparatus was previously developed by the researcher during his master's thesis [144].

\subsubsection{Implementation Details}

The following reference model is used to implement the admittance controller:

$$
K_{1} \ddot{q}_{r}=\tau_{\text {int }},
$$

where $q_{r}$ is the device reference trajectory, $\tau_{i n t}$ is the interaction torque, and $K_{1}$ specifies the desired inertia of the device. As noted in [115], adding damping in the reference model helps stabilize the interaction. Thus, excluding a damping term in the reference model generates a more challenging condition for testing the impedance manipulations.

The parameters $\psi, \gamma$, and $\eta$ are held constant during all experiments at $\psi=25 \frac{\mathrm{rad}}{\mathrm{s}^{2}}$,

$\gamma=500 \frac{1}{\mathrm{~s}}$, and $\eta=600 \frac{\mathrm{rad}}{\mathrm{s}^{2}}$. A first-order, low-pass filter with a bandwidth of $12 \mathrm{rad} / \mathrm{s}$ is used to filter $\dot{s}$ before (3.28) is evaluated. When the acceleration limits are used, the reference trajectory $q_{r}$ is realized by integrating $\ddot{q}_{t}$ from (3.28) after it has been limited to lie between (3.9) and (3.10). This process is summarized in Figure 3.1.

\subsubsection{Bounding Tracking Errors by Limiting the Reference Acceleration}

During this experiment, the participant was requested to perform an abrupt forearm extension with the haptic and to hold his forearm position constant after the extension. 
The rapid extension represents an example of a highly dynamic motion that could be observed in practice (e.g., when a user executes a rapid limb motion with a haptic device to avoid an obstacle in the environment). Such dynamic motions cause a rapid change in the reference trajectory that can be difficult for the motor to track. Previous experience suggests, as shown below, that such rapid motions can suddenly destabilize the interaction. Thus, the torque limits of the actuator were reduced to a safe amount to ensure that the there was no risk of harm to the user.

The experiments were repeated with and without the acceleration limits active at 6 different PD controller settings. The desired inertia of the device $K_{1}$ was specified as 0.2 $\mathrm{kgm}^{2}$ during all experiments. The PD controller gains were selected to reflect various combinations of low, medium, and high proportional $(\mathrm{P})$ and derivative $(\mathrm{D})$ gain. This experiment investigated whether the acceleration limits could ensure stability under a wide range of position controller gains.

High $\mathrm{P}$ and $\mathrm{D}$ gains were defined as the largest $\mathrm{P}$ and $\mathrm{D}$ gains that did not induce any noticeable chatter. The gain settings, and tracking errors measured at each $\mathrm{P}$ and $\mathrm{D}$ gain combination are summarized in Table 3.1. The theoretical error bound shown in Table 3.1 was calculated using (3.18), and only applies to the experiments performed with the acceleration limits active.

The results in Table 3.1 show that the interaction was unstable and exhibited peak tracking errors exceeding 590 degrees at all gain settings when the reference acceleration limits were not used. The tracking error data shown in Figure 3.4a also confirms that the interaction was suddenly destabilized after the onset of the subject's extension approximately 2 seconds into each test run. It is important to highlight that the interaction is not immediately unstable (i.e., tracking errors are bounded between 0 and 2 seconds when the user is slowly moving the device). The user's sudden extension instigates the instability. This supports the previous conjecture that this form of instability is insidious in that 
Table 3.1: Comparing tracking errors with and without the acceleration limits active

\begin{tabular}{|c|c|c|c|c|}
\hline $\begin{array}{c}\text { P Gain } \\
(\mathrm{Nm} / \mathrm{rad})\end{array}$ & $\begin{array}{c}\text { D Gain } \\
(\mathrm{Nm} \cdot \mathrm{s} / \mathrm{rad})\end{array}$ & $\begin{array}{c}\text { Error } \\
\text { Bound (deg) }\end{array}$ & $\begin{array}{c}\text { Max. Error - Accel. } \\
\text { Limits Active (deg) }\end{array}$ & $\begin{array}{c}\text { Max. Error - Accel. } \\
\text { Limits Inactive (deg) }\end{array}$ \\
\hline 10000 & 50 & 3.44 & 0.33 & 790.74 (unstable) \\
10000 & 250 & 3.44 & 1.67 & 658.16 (unstable) \\
2500 & 50 & 13.75 & 1.34 & 1075.5 (unstable) \\
2500 & 250 & 13.75 & 6.09 & 593.01 (unstable) \\
250 & 50 & 137.51 & 1.32 & 761.14 (unstable) \\
20000 & 50 & 1.72 & 0.17 & 930.93 (unstable) \\
\hline
\end{tabular}

typical clues such as persistent oscillations are not present from the onset, and nothing otherwise suggests that the interaction could be destabilized after the extension. It should be noted that the saturation limits of the actuator were purposely restricted to ensure the user could resist the orthosis. If the current limits were increased, then the orthosis would violently oscillate and pose a serious risk of injury for the user.

In contrast, Table 3.1 and Figure $3.4 \mathrm{~b}$ shows that the interaction remained stable and that the peak tracking error remained below the value predicted by (3.18) - at all $\mathrm{P}$ and $\mathrm{D}$ gain combinations, when the acceleration limits were used. Thus, these results confirm that the acceleration limits can stabilize the interaction in situations where a conventional controller may fail. These results also confirm that the acceleration limits provide a predictable tracking error response consistent with Theorem 1.

Static reference acceleration limits could also be used to help limit rapid changes in the reference trajectory. Such limits may help prevent instabilities in some cases. However, static acceleration limits do not provide a proven stability guarantee, and may severely limit the haptic device's ability to accelerate quickly. In contrast, (3.9) and (3.10) ensure that the tracking error is bounded, and can easily be tuned to achieve the desired trade-off between minimizing tracking error and allowing large device accelerations. 


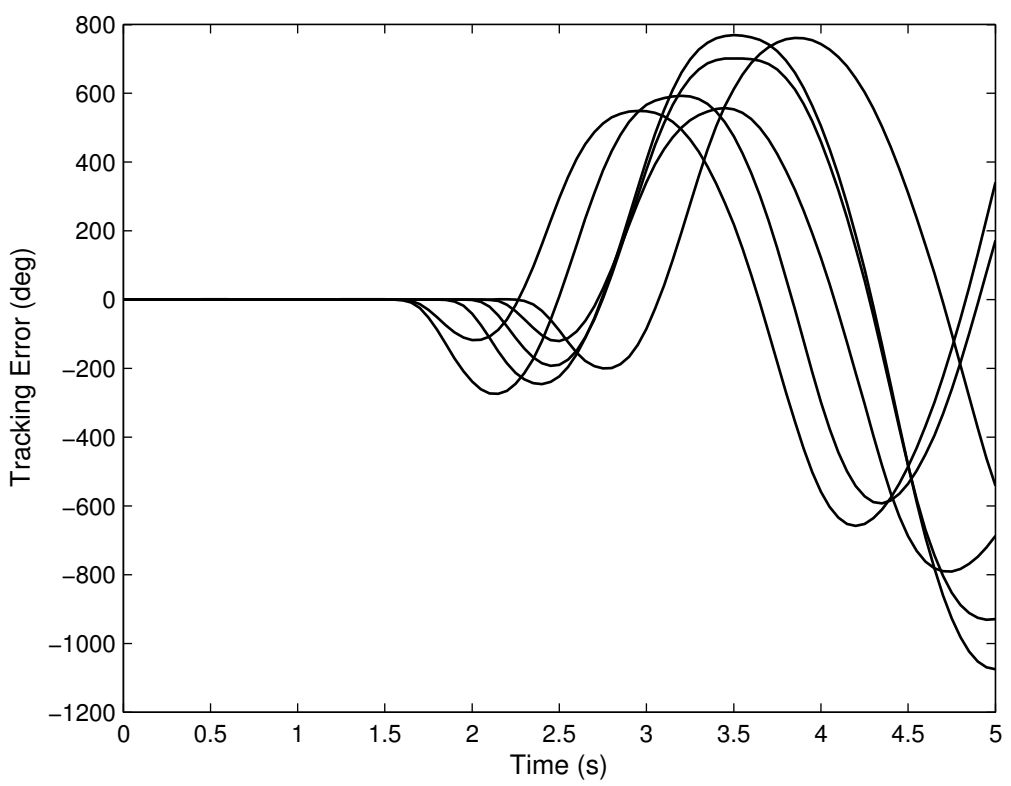

(a)

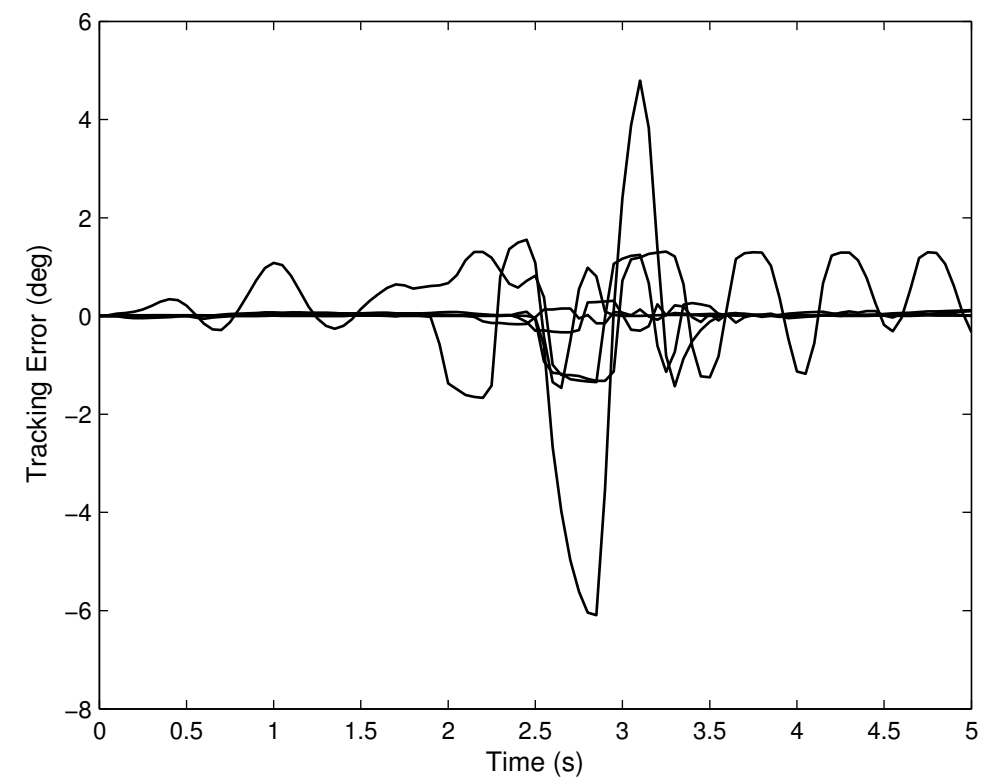

(b)

Figure 3.4: Tracking error response when the user performs abrupt forearm extension at six different PD controller gain settings. (a) Without acceleration limits active. (b) With acceleration limits active. Without the acceleration active, the interaction becomes unstable whenever the subject abruptly extends his forearm. No instabilities are observed when the acceleration limits are active. 


\subsubsection{Stability Under Actuator Saturation}

Solutions for addressing the effects of actuator saturation remains an ongoing research topic in the control community $[134,135,145-148]$. As noted in these works, saturation can degrade performance, and may even lead to instability in some situations. The experiments discussed in this section present a scenario where actuator saturation leads to instability, and show that the reference acceleration limits guarantee stable interactions under saturation.

During the experiments, the subject performed consecutive, abrupt forearm extensions while attached to the orthosis to try and destabilize the control system. The first data collection test run was performed with a conventional PD controller implemented and with the motor amplifier current limited to \pm 1 A (i.e., the $1 \mathrm{~A}(\mathrm{PD})$ test condition). The second test run was performed with a PD controller and with amplifier current limits increased to $\pm 2.5 \mathrm{~A}$ (i.e., the $2.5 \mathrm{~A}(\mathrm{PD})$ test condition). A final data collection test run was then

performed with the reference acceleration limits active and a reduced amplifier current restriction of $\pm 1 \mathrm{~A}$ (i.e., the $1 \mathrm{~A}(\mathrm{PD}+\mathrm{AL})$ test condition). The first two test runs were performed to investigate whether actuator saturation can destabilize the interaction. The final test run was performed to confirm that the acceleration limits are immune to saturation-instigated instabilities.

The reference model parameters were held constant at $K_{1}=0.2 \mathrm{kgm}{ }^{2} \mathrm{Nm} \cdot \mathrm{s} / \mathrm{rad}$ during all experiments. P and D gains of $10000 \mathrm{Nm} / \mathrm{rad}$ and $50 \mathrm{Nm} \cdot \mathrm{s} / \mathrm{rad}$ were used in all tests. These nominal gain values were selected because preliminary experiments indicated that they resulted in an oscillation-free interaction. At lower desired inertia settings, a non-zero value of $b$ (i.e., some error feedback) would be necessary for attenuating oscillations.

The instability of the $1 \mathrm{~A}$ (PD) controller setting can be seen in Figure 3.5a. This instability is characterized by the sudden divergence of the tracking error 5.7 seconds into 


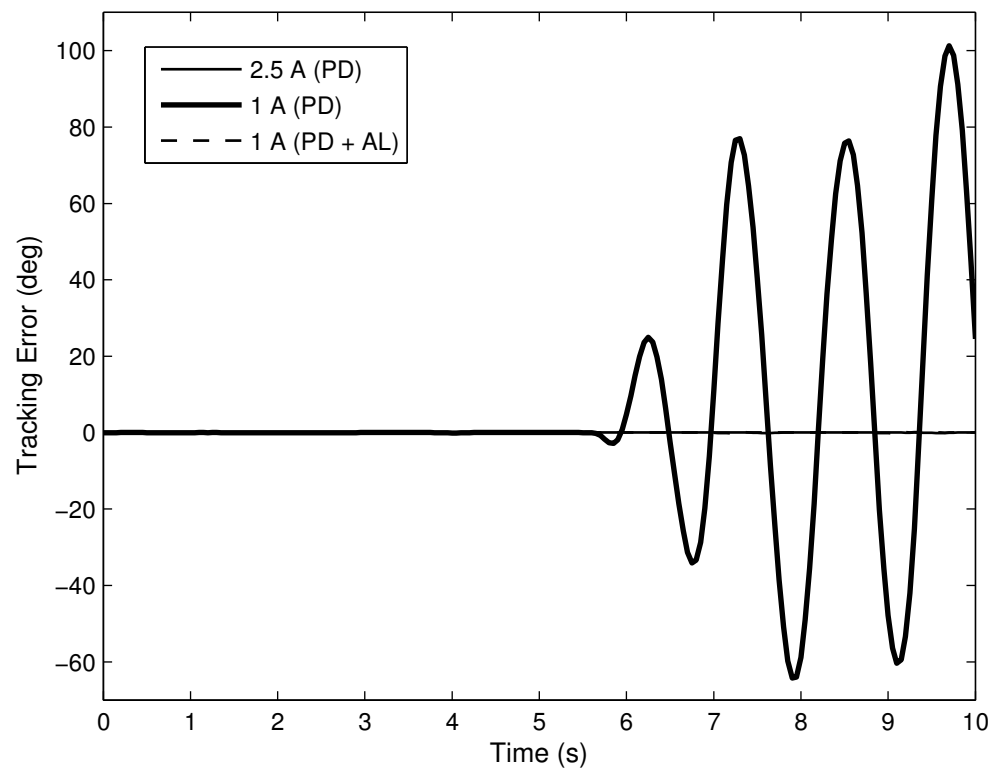

(a)

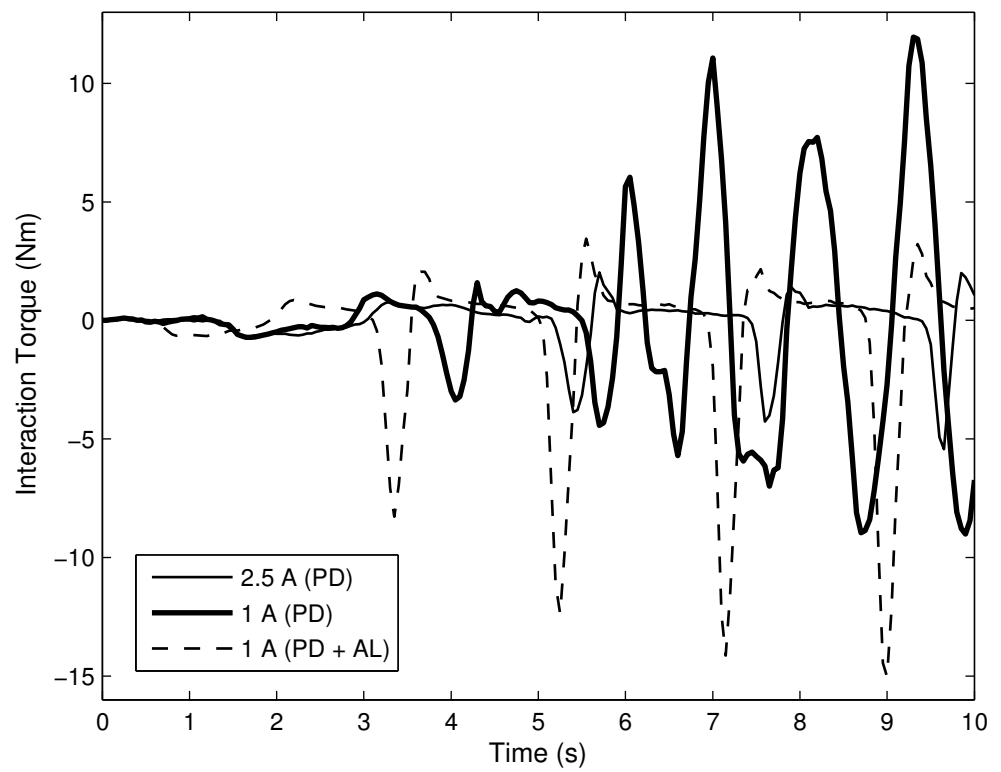

(b)

Figure 3.5: Using acceleration limits to eliminate actuator saturation-instigated instabilities. Captions are continued on the following page. 


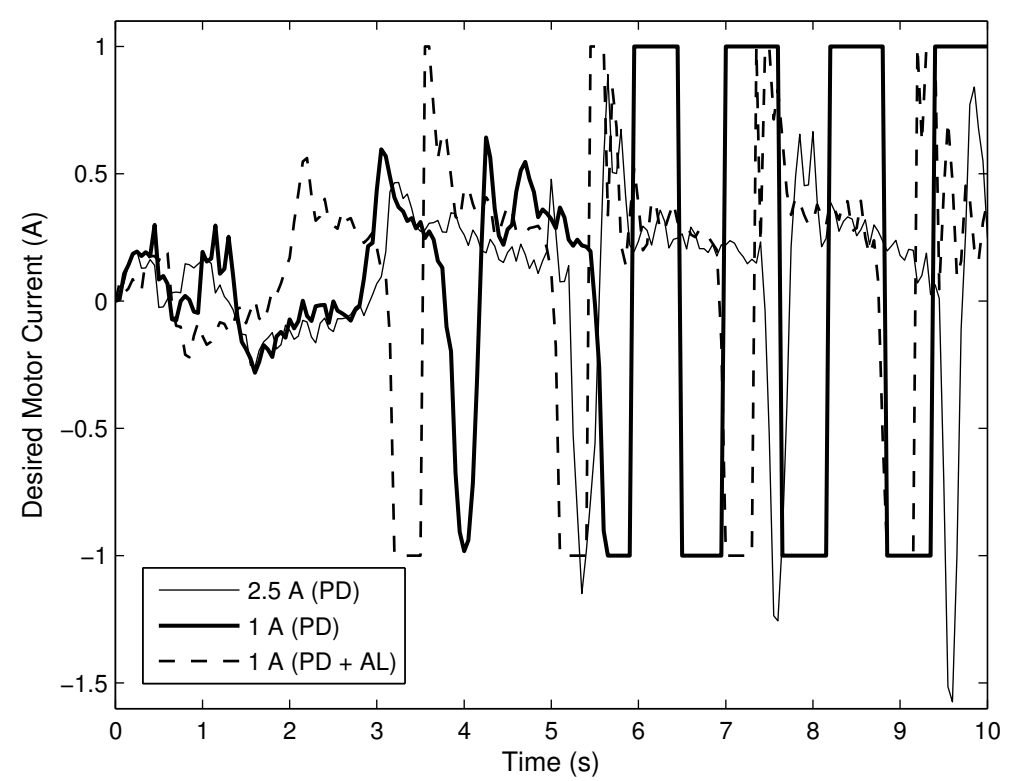

(c)

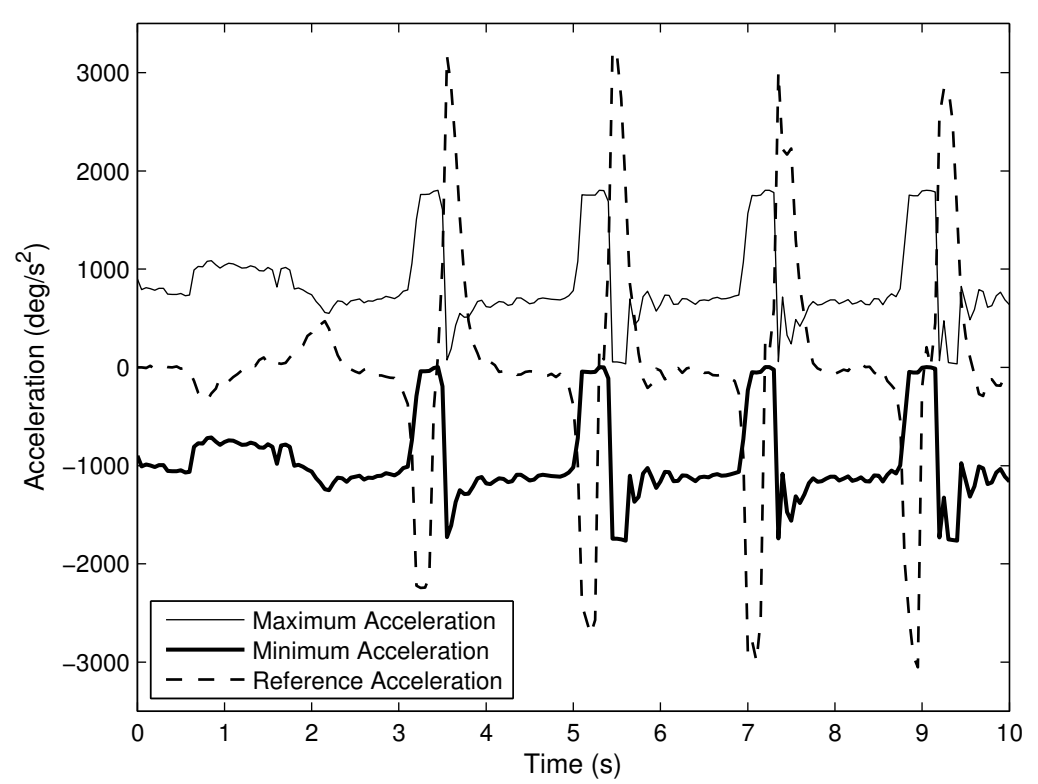

(d)

Figure 3.5: Using acceleration limits to eliminate actuator saturation-instigated instabilities. (a) Interaction torque. (b) Tracking error. (c) Desired motor current. (d) Acceleration limit activations. Stability is guaranteed irrespective of whether the actuator saturates or not, if the reference acceleration limits are used. Also, the reference acceleration is only limited when the interaction is on the verge of instability. 
the test. Figure 3.5b, which plots the measured interaction torque from all test conditions, confirms the that interaction was unstable; this figure shows that the interaction torque continued to oscillate uncontrollably after the user performed the second abrupt extension at the $1 \mathrm{~A}(\mathrm{PD})$ test condition. In contrast, the Figure 3.5a shows that the tracking error remained bounded throughout the $2.5 \mathrm{~A}(\mathrm{PD})$ test condition.

Figure 3.5c, which plots the motor current command from all three test runs, confirms that the onset of instability in the $1 \mathrm{~A}(\mathrm{PD})$ test condition is coincident with the point at which the actuator first saturates. As no other controller parameters were varied, and since the user performed similarly rapid forearm extensions at both the $1 \mathrm{~A}(\mathrm{PD})$ and 2.5 A (PD) test conditions, the instability of the interaction at $1 \mathrm{~A}(\mathrm{PD})$ test condition can be attributed to the saturation of the actuator. These results also demonstrate that the instabilities due to saturation are difficult to predict. Figure 3.5a shows that there are no prior warnings such as sustained oscillation to suggest that the interaction might become unstable. In fact, the user's first extension at $1 \mathrm{~A}(\mathrm{PD})$ test condition at $4 \mathrm{~s}$ did not even destabilize the interaction.

The results in Figure 3.5b also confirm that the acceleration limits were capable of guaranteeing a stable interaction and bounded tracking error at the $1 \mathrm{~A}(\mathrm{PD}+\mathrm{AL})$ test condition. The peak negative interaction torques at the $1 \mathrm{~A}(\mathrm{PD}+\mathrm{AL})$ test condition were significantly higher than at all other conditions. This implies that the reference acceleration limits were effective at stabilizing the interaction even when the user performed significantly more forceful extensions. Moreover, the acceleration limits guaranteed bounded tracking error and a stable interaction even when the actuator was saturated (e.g., between 3.2 and 3.5 seconds in Figure 3.5c).

The variation of the acceleration limits during the $1 \mathrm{~A}(\mathrm{PD}+\mathrm{AL})$ test condition is plotted Figure 3.5d. A comparison of Figures 3.5a and 3.5d confirms that the acceleration limits only restricted the device's maximum acceleration during the subject's forceful extensions. 
Gauaging from the limits varied in the figure, highly conservative acceleration limits would be required to guarantee a stable interaction if static reference acceleration limits were used.

\subsubsection{Oscillation Attenuation via Error Feedback}

Admittance-controlled haptic devices are prone to instability or oscillations when commanded to display a small impedance $[109,111,115,116]$. This experiment was performed to illustrate how error feedback in the reference acceleration (3.9), and the acceleration limits (3.10) and (3.28) together, help overcome this limitation that conventional admittance controllers face.

The experiment protocol required that the subject track a sinusoidal forearm trajectory at different controller settings. As shown in Figure 3.6, a monitor displayed the desired and measured device orientations in real-time. The subject performed the tracking task by keeping the distance between both lines a minimum at all times. Asking the subject to accurately track the desired trajectory ensured that the subject repeated a similar forearm motion at all test conditions.

The desired inertia of the device was set to $K_{1}=0.005 \mathrm{kgm}^{2}$, and $\mathrm{P}$ and $\mathrm{D}$ gains of $10000 \mathrm{Nm} / \mathrm{rad}$ and $50 \mathrm{Nm} \cdot \mathrm{s} / \mathrm{rad}$ were used in all tests. The subject performed a total of four test runs corresponding to four unique values of $b$ in (3.28). Parameter values and select results are summarized in Table 3.2 and plotted in Figure 3.7.

Figure 3.7a plots the measured device orientations from all test runs. The dark line in Figure 3.7a denotes the desired sinusoidal trajectory. The remaining lines show the measured device orientations. These results confirm that the user performed a similar forearm motion at each test condition.

Section 3.3 suggested that a haptic device may exhibit sustained oscillations when 


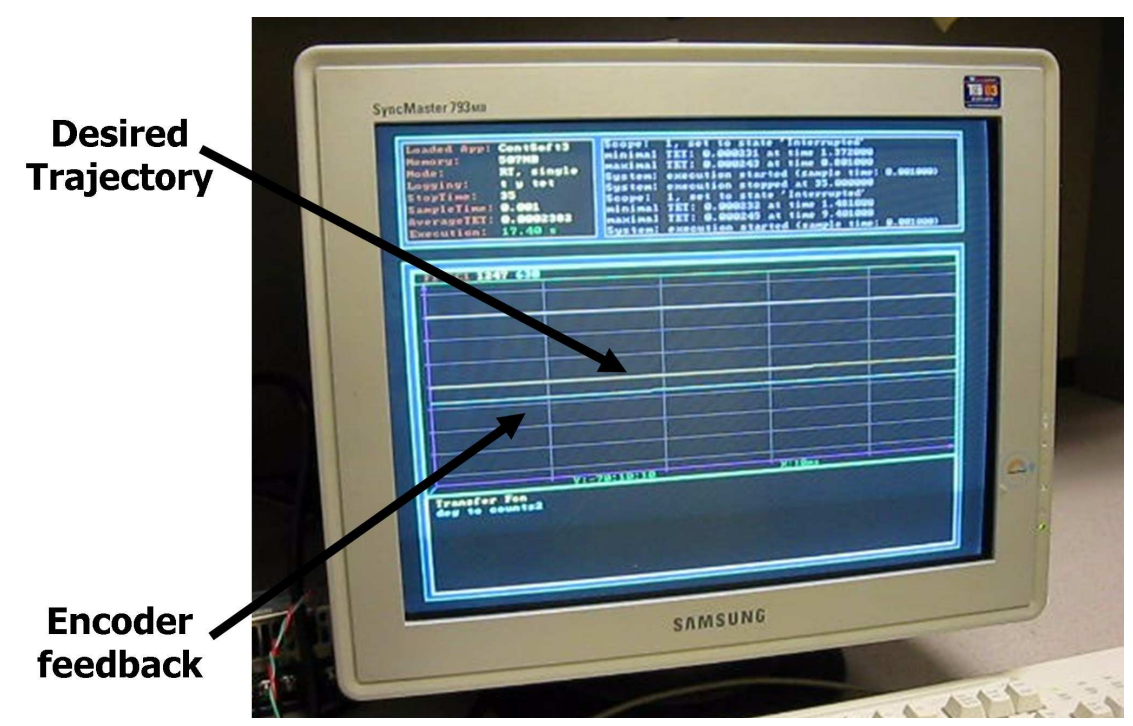

Figure 3.6: An image of monitor providing real-time feedback of the desired and measured forearm orientation. Users were instructed keep the distance between both lines a minimum at all times when performing tracking tasks.

required to display a small, pure mass. Figure $3.7 \mathrm{~b}$, which plots the power spectral density (PSD) estimate of the interaction torque signal for $b=0$ and $b=40$, shows an example of this behaviour. When $b=0$, i.e., with (3.28) inactive, the interaction torque is highly oscillatory and exhibits high energy peaks at 14, 25, and $40 \mathrm{~Hz}$. The oscillations at the $b=0$ test condition were strong enough to vigorously shake the user's forearm for the duration of the test. Since the frequency of the user's voluntary motion was approximately $0.1 \mathrm{~Hz}$, these high-frequency peaks cannot be attributed to the user's voluntary motion.

Table 3.2: Reducing oscillations by using error feedback in the reference model

\begin{tabular}{|c||c|c|}
\hline $\mathrm{b}$ & RMS $\tau_{\text {int }}$ & RMS $\dot{V}$ \\
\hline 0 & 0.722 & 45.51 \\
12.5 & 0.263 & 15.02 \\
25 & 0.036 & 0.07 \\
40 & 0.023 & 0.01 \\
\hline
\end{tabular}

Table 3.2 also shows that the root mean square (RMS) value of the interaction torque, RMS $\tau_{\text {int }}$, decreased as $b$ was increased from 0 to 40 . Figure $3.7 \mathrm{~b}$ further shows that the 
most significant peak in the PSD estimate at $14 \mathrm{~Hz}$ is attenuated by more than $40 \mathrm{~dB}$ when $b=40$. Both pieces of information confirm that the error feedback was effective at attenuating oscillations. Moreover, as noted in Section 3.3.1, re-shaping the reference acceleration should reduce the maximum positive magnitude of $\dot{V}$. The results in Table 3.2 also demonstrate this, and show that the RMS of $\ddot{V}$ decreased significantly as $b$ was increased.

Figure 3.7c plots the interaction torque for the $b=40$ test run. This figure confirms that the user had to apply a negligible torque input to perform tracking task. Table 3.2 further shows that the RMS interaction torque during the $b=40$ test run was only 0.023 Nm. The minimal torque input required to move the device was analogous to the subject performing the same motion while holding onto a wooden pencil. Accordingly, these results confirm that the impedance manipulations allow an admittance-controlled haptic device to safely display a very small impedance.

Additional experiments demonstrated that the impedance manipulations enabled stable interactions at desired inertia settings as low as $K_{1}=0.001 \mathrm{kgm}^{2}$. However, oscillations were more difficult to attenuate at such low apparent inertia settings since the effects of sensor noise became increasingly more apparent. At the $b=40$ test condition, for example, the RMS of $\tau_{i n t}$ was $0.023 \mathrm{Nm}$. However, the RMS of the noise in $\tau_{\text {int }}$ signal was calculated to be one-third of this value at $0.0072 \mathrm{Nm}$. Thus, even though the impedance manipulations allowed stable displays of lower apparent inertias, users always had to modulate their input to compensate for the effects of sensor noise.

Sensor noise imposes a barrier on the minimum allowable impedance display, and inevitably reduces the fidelity of the impedance display. The RMS of $\tau_{i n t}$ divided by the RMS of $\ddot{q}_{r}$ provides an estimate of the average inertia that the haptic device displays during a test run. Using the RMS of $\ddot{q}_{r}$ instead of the RMS of $\ddot{q}$ is justified since the small tracking errors seen in Figure 3.7d confirm that the device accurately displayed the 


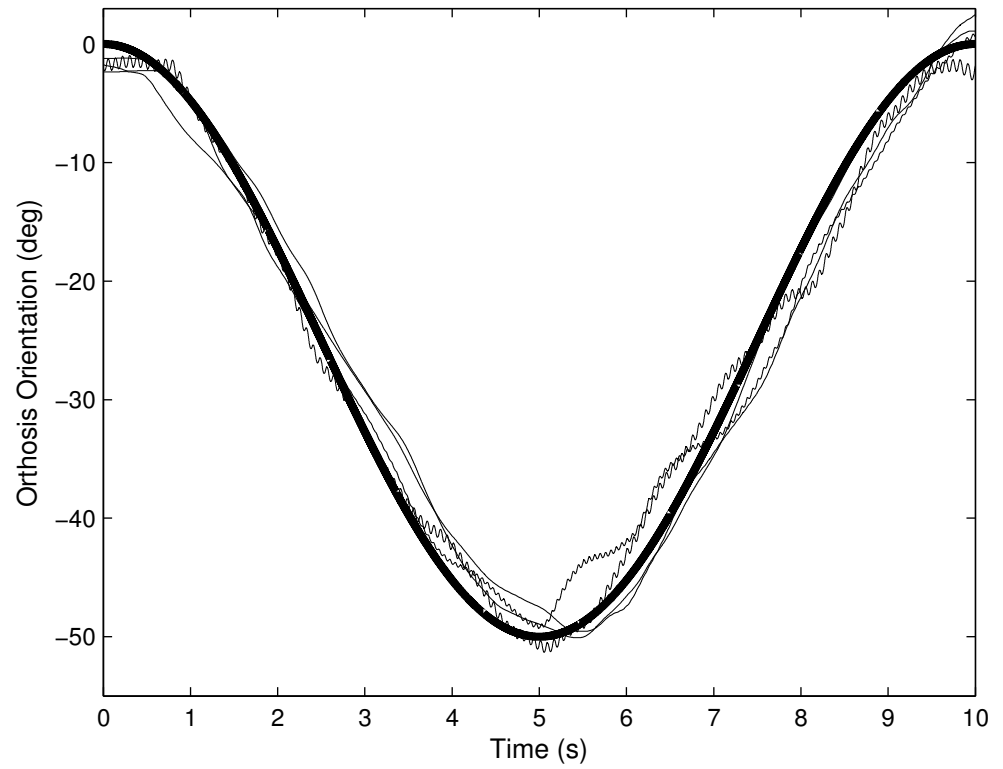

(a)

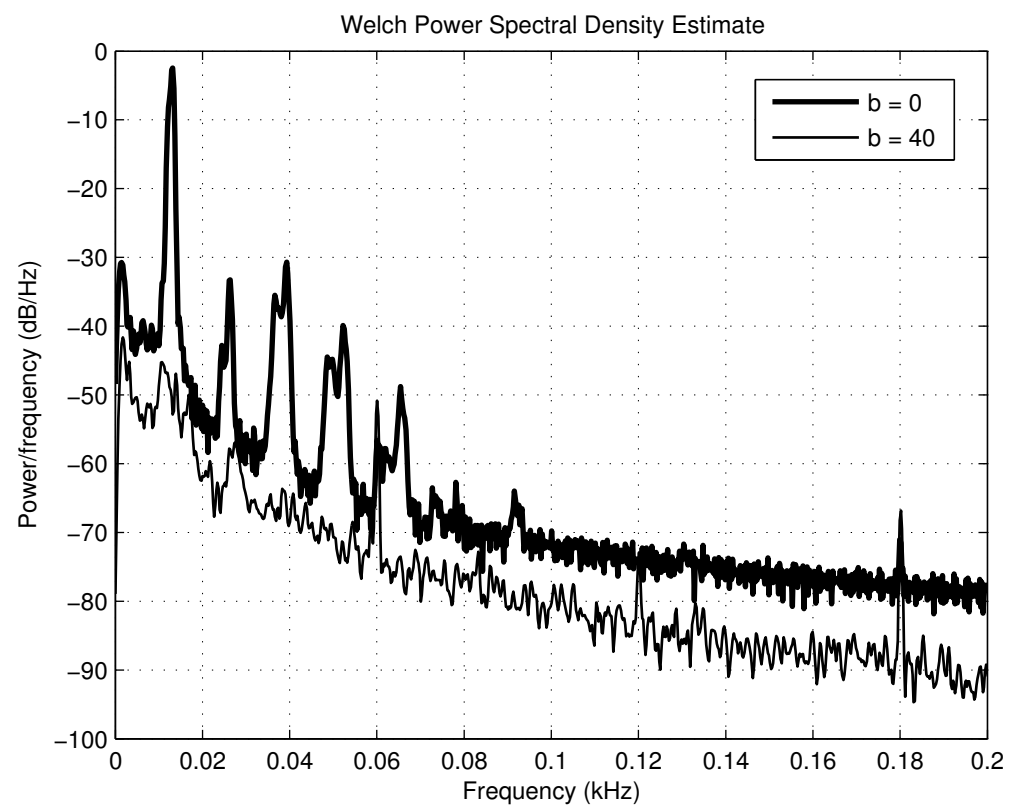

(b)

Figure 3.7: Safely displaying a 99.3\% apparent inertia reduction. Captions are continued on the following page. 


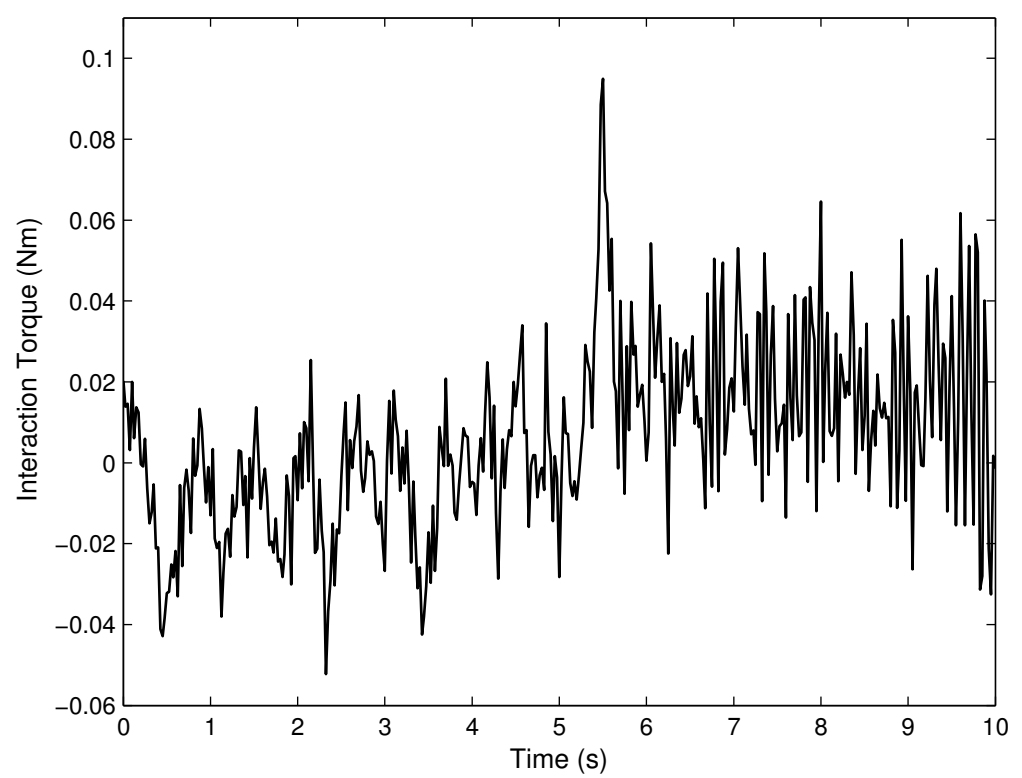

(c)

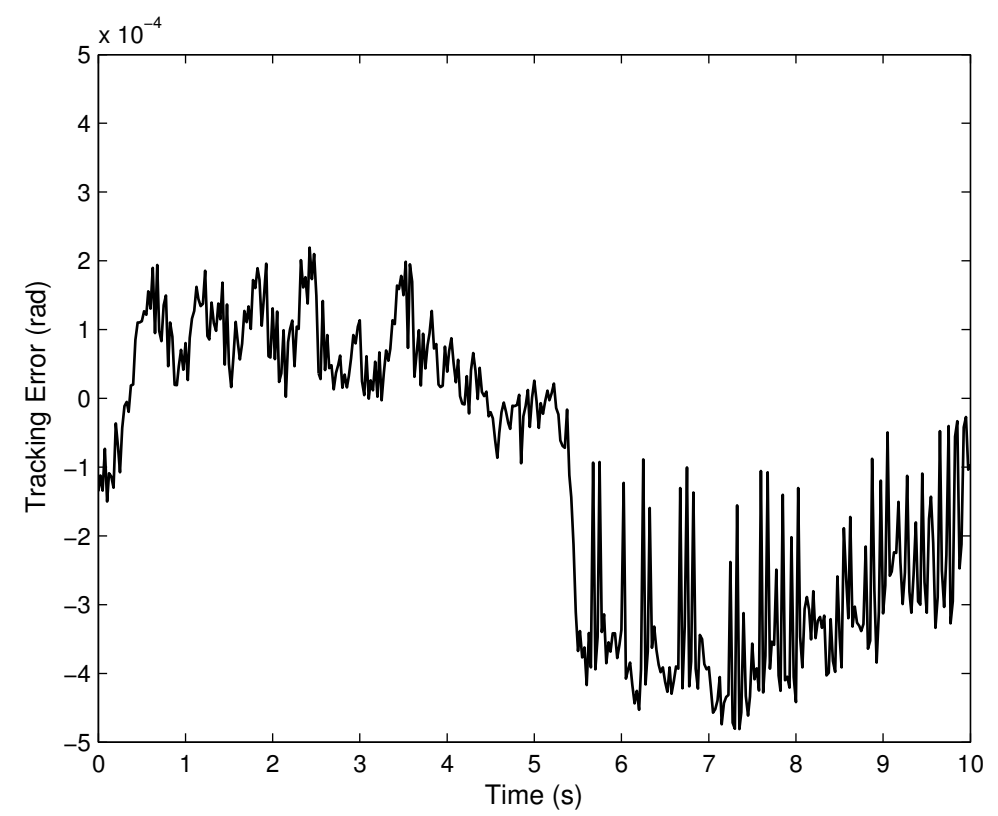

(d)

Figure 3.7: Safely displaying a 99.3\% apparent inertia reduction. (a) Device orientation. (b) Power spectral density estimate of interaction torque. (c) Interaction torque during the $b=40$ test run (d) Tracking error during the $b=40$ test condition. With error feedback active (i.e., when $\mathrm{b}=40$ ), oscillations were sufficiently attenuated and the subject could manipulate the device with a small torque input. 
reference dynamics; the figure shows that the peak tracking error was less than 0.0005 rad. For the $b=40$ test condition, the average inertia displayed was calculated to be 0.0074 $\mathrm{kgm}^{2}$. This represents a $48 \%$ error with respect to the desired inertia of $0.005 \mathrm{kgm}^{2}$.

While the accuracy of the impedance display is not ideal, the results confirm that the impedance manipulations allowed the device to display an apparent inertia reduction of 99.3\%. To the authors' knowledge, the largest published apparent mass/inertia reduction in the literature is only $98 \%$ [120]. In general, the impedance manipulations allow higher apparent mass/inertia reductions in comparison to results published in [120] (98\% reduction), [129] (89\% reduction), [149] (82\% reduction), and [119] (80\% reduction). It is important to note that the seemingly marginal gain obtained by using the impedance manipulations can be quite significant when the overall mass of the haptic device increases, such as for a heavy haptic gait trainer like the GaitEnable device described in Chapters 4 and 5 .

In addition to sensor noise, actuator bandwidth and torque limitations also need to be considered. Again, even if the impedance manipulations allow stable display of a 99.999\% apparent inertia reduction, the actual apparent inertia reduction displayed may be significantly lower if the motor cannot generate enough torque to accelerate the device,

or if the actuator's or position controller's bandwidth is insufficient to keep up with the user's rapid motion. Moreover, the actual apparent inertia reduction that can be achieved will depend on the user's motion. The device will feel lighter at slow motions of the type considered in this experiment, but heavier during dynamic motions that saturate the actuators, or exceed the bandwidth of actuator/position controller.

\subsubsection{Stable Interactions during Dynamic Device Motions}

The previous experiments illustrated how (3.28), (3.9), and (3.10) allow the 1-DOF haptic device to safely display a small inertia. As the previous experiments only considered 
slow and gentle motions, additional experiments were performed to investigate what happens when the user rapidly accelerates the device. Rapid accelerations are problematic because they can lead to instability (see Section 3.4.3), and because they can cause a sustained activation of the acceleration limits that significantly alters the target impedance.

The experiment protocol required the subject to perform a rapid extension at 2 different values of $K_{1}$ and $b$. The initiation of the extension was triggered by a visual cue on a computer monitor that prompted the user to perform a sudden extension of 40 degrees. The monitor also provided the user with real-time feedback of the measured and desired device orientations. The subject used this feedback to control his forearm orientation.

The subject was simply instructed to extend his forearm as quickly as possible, and to try and avoid any overshoot. The first set of extensions were performed with $K_{1}$ and $b$ set to $0.5 \mathrm{kgm}^{2}$ and 0 , respectively; $b$ could be set to zero since the interaction was not oscillatory at this desired inertia setting. The second set of extensions were performed with $K_{1}$ and $b$ set to $0.005 \mathrm{kgm}^{2}$ and 40 , respectively. Multiple test runs were performed at each parameter setting since it was difficult for the subject to perform the tracking task without overshoot. P and D gains of $10000 \mathrm{Nm} / \mathrm{rad}$ and $50 \mathrm{Nm} \cdot \mathrm{s} / \mathrm{rad}$ were used in all tests.

Data from each parameter setting showing the greatest similarity in the subject's forearm motion were selected for the comparison shown in Figure 3.8. Comparing data sets for similar device motions was necessary for isolating how $K_{1}$ and $b$ influence the interaction torque required to perform the extension. Additional data from the experiments can also be found in Table 3.3.

The quantity $P_{r e f}$ in Table 3.3 is the product of $\tau_{i n t}$ and $\dot{\theta}_{r}$. It denotes the instantaneous power that user supplies to the reference model. $E_{r e f}$, the energy supplied to the reference model, is calculated by integrating $P_{\text {ref }}$ for the duration of the test $(3 \mathrm{~s})$. Both $P_{\text {ref }}$ and $E_{\text {ref }}$ should be nearly equal when the user repeats a specific motion at 


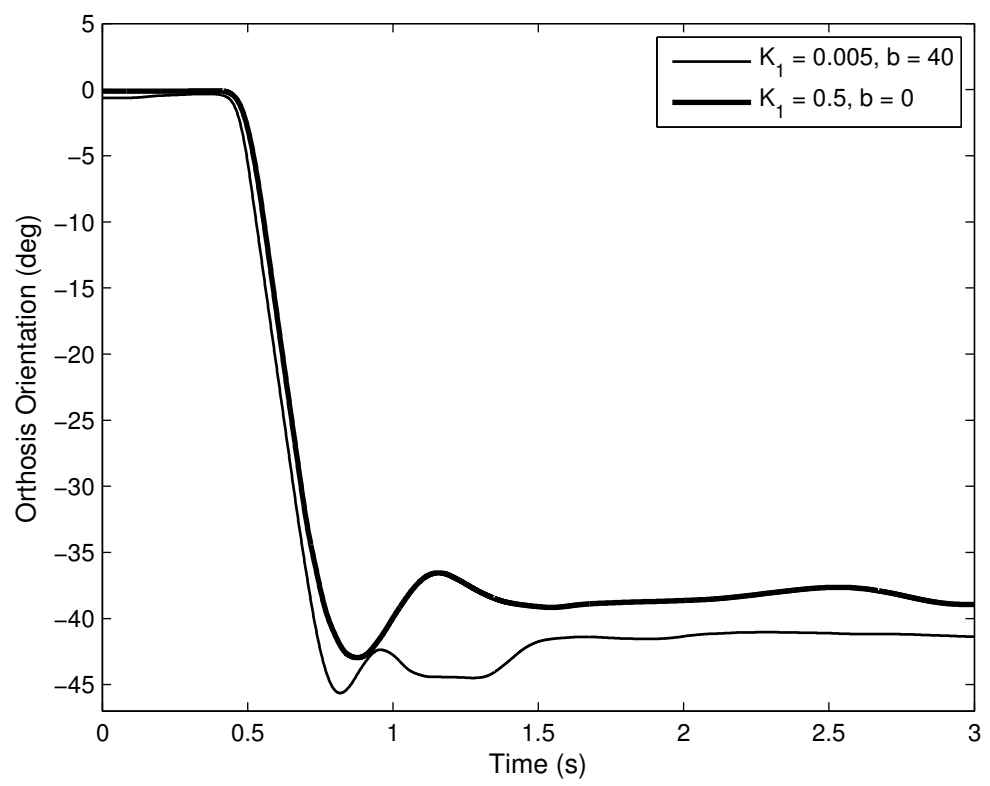

(a)

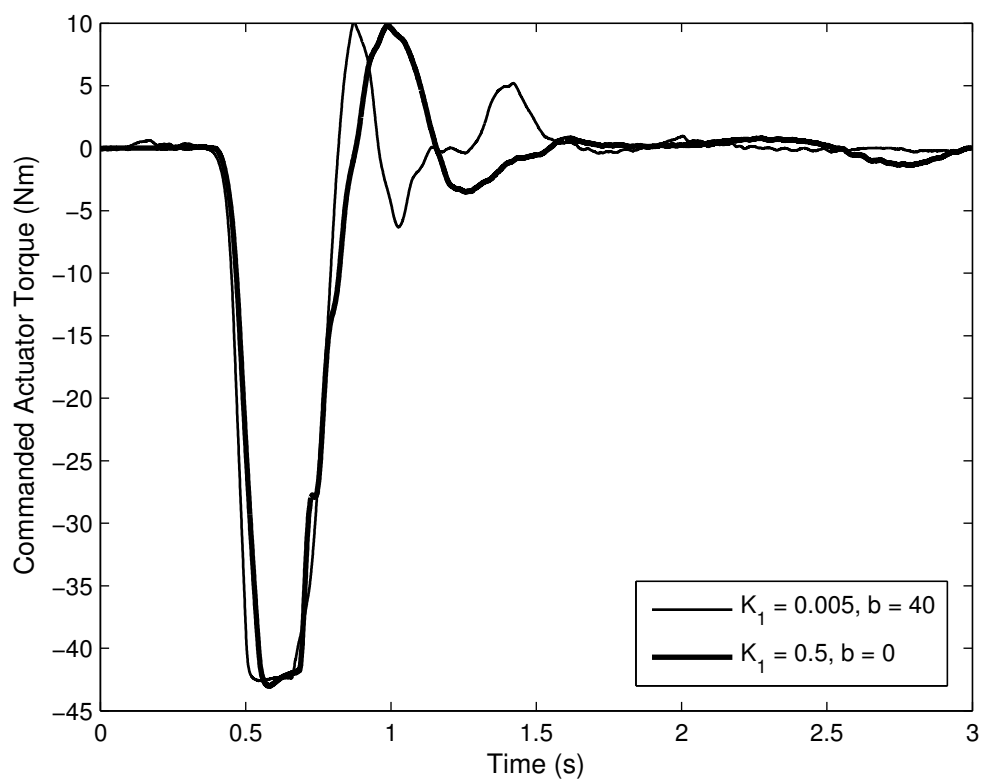

(b)

Figure 3.8: Improved performance during dynamic motions. Captions are continued on the following page. 


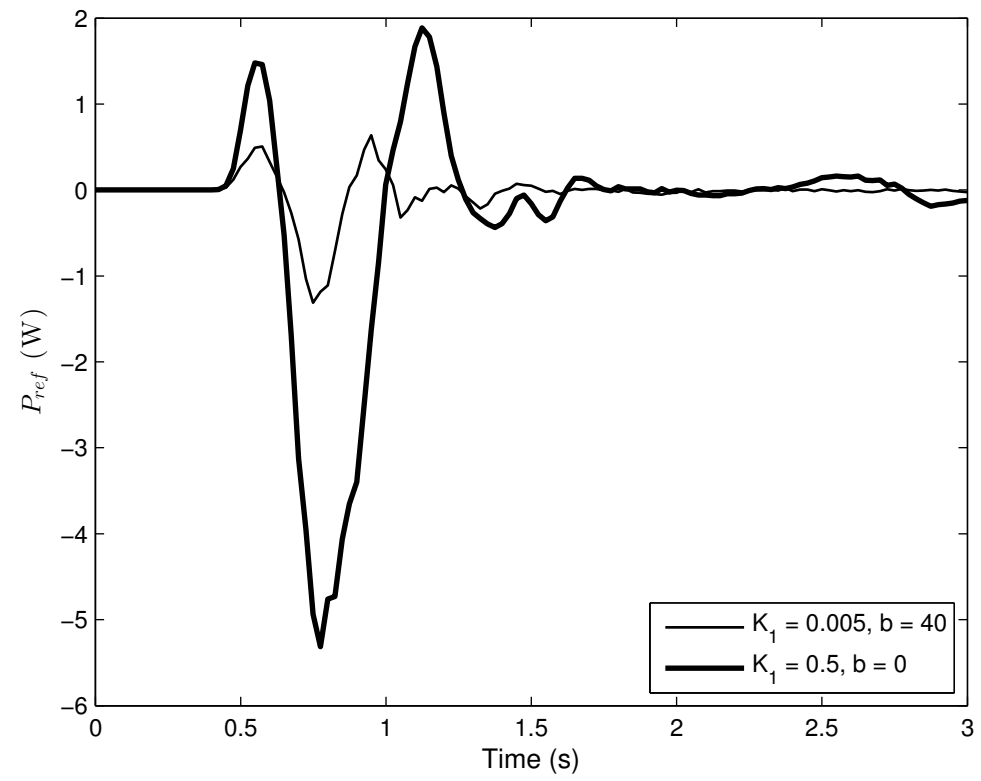

(c)

Figure 3.8: Improved performance during dynamic motions. (a) Device orientation. (b) Commanded actuator torque. (c) Power supplied to reference model. Though the subject's motion, and the actuator command signals are qualitatively similar at both test conditions, the power input into the reference model is significantly smaller when the desired inertia is set to a lower value.

Table 3.3: Dynamic motions at high apparent inertia reduction settings

\begin{tabular}{|c|c|c|c|}
\hline$\left(K_{1}, b\right)$ & $\begin{array}{c}\text { Peak } P_{\text {ref }} \\
(\mathrm{W})\end{array}$ & $\begin{array}{c}E_{\text {ref }} \\
(\mathrm{J})\end{array}$ & $\begin{array}{c}\mathrm{RMS} \tau_{\text {int }} \\
(\mathrm{Nm})\end{array}$ \\
\hline$(0.5,0)$ & -5.34 & -0.77 & 2.95 \\
$(0.005,40)$ & -1.31 & -0.10 & 0.59 \\
\hline
\end{tabular}


a given parameter setting. Reducing the value of the $K_{1}$ should make the device easier to move, and should result in a decreased value of $P_{r e f}$ and $E_{r e f}$ when a motion is repeated. Accordingly, both $P_{r e f}$ and $E_{r e f}$ provide a quantitative measure of the user's effort.

Figure 3.8a plots the measured device orientation from both data sets. These results demonstrate that subject was capable of rapidly accelerating the device without destabilizing the interaction. This figure also shows that both extensions resulted in qualitatively similar actuator commands. However, Figure 3.8c shows that there is a significant decrease in $P_{\text {ref }}$ when $K_{1}$ was set to $0.005 \mathrm{kgm}^{2}$. Table 3.3 also shows that the peak value of $P_{\text {ref }}$, $E_{r e f}$, and the RMS value of the interaction torque were reduced by factors of 4,7 , and 5 , respectively, at the lower inertia setting. The fact that the extension was easier to perform at the lower inertia setting is not surprising in and of itself. However, this result is noteworthy because it confirms that the overall behaviour of the device remains predictable as the desired impedance of the device is varied; one can modify the parameters of the reference model and expect to see corresponding changes in the behaviour of the device even if the impedance manipulations temporarily interfere with the impedance display during highly dynamic motions.

\subsubsection{Experiments with Multiple Healthy Subjects}

These experiments were performed with a total of 10 healthy male and female subjects ranging in age from 22 to 44 years with the goal of investigating whether subject-specific differences (e.g., different body morphologies or ways of interacting with the device) can result in unstable interactions. All subjects repeated the experiments at an inertia setting of $K_{1}=0.005 \mathrm{kgm}^{2}$ and error feedback parameter $b$ of $40 . \mathrm{P}$ and D gains of $10000 \mathrm{Nm} / \mathrm{rad}$ and $50 \mathrm{Nm} \cdot \mathrm{s} / \mathrm{rad}$ were used in all tests. The choice to use identical parameters for all users also provided a means for investigating whether subject-specific parameter tuning was necessary.

Each subject was requested to perform a rapid forearm extension of 40 degrees with 


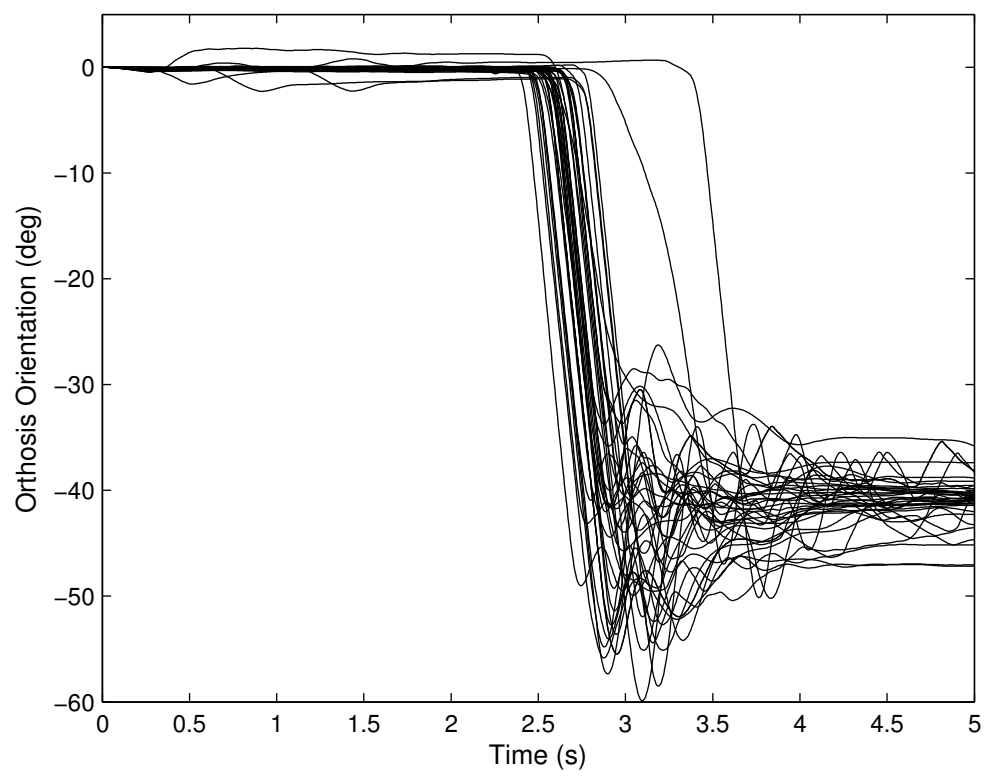

(a)

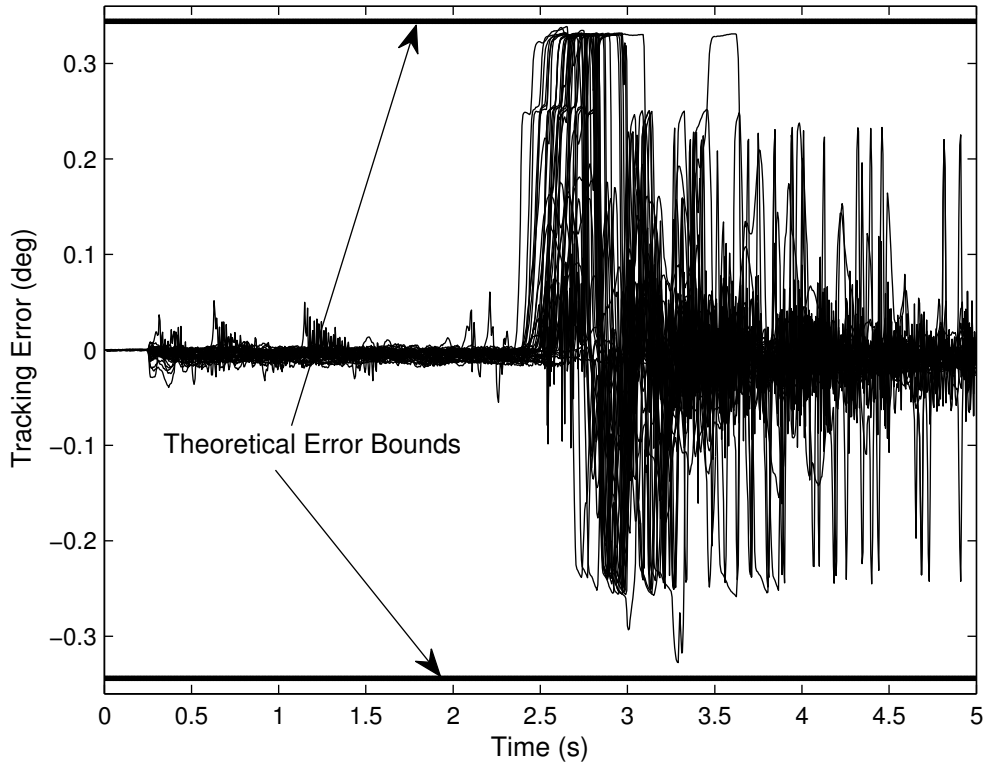

(b)

Figure 3.9: Rapid extensions performed by healthy subject. (a) Device orientation (all users). (b) Tracking error (all users). Subjects were able to rapidly extend their forearms without destabilizing the interaction. The impedance manipulations guarantee the stability of the position control loop during all test runs. 
the device. The initiation of the extension was triggered by a visual cue on a computer monitor. The monitor also provided the user with real-time feedback of the measured and desired device orientations. As in the previous experiment, subjects were simply instructed to extend their forearm as quickly as possible, and to try and avoid overshoot. Specifying a low desired inertia and asking subjects to perform a rapid motion created a worst-case scenario for experimentally evaluating the stability of the impedance manipulations. As shown previously, conventional controllers cannot generate unstable interactions under this condition.

Each subject performed a minimum of 5 repetitions before data collection to ensure that they were familiar with behaviour of the device. Data were collected from a total of 40 test runs (3-5 test runs per subject). Selected results are presented in Figure 3.9.

Figure 3.9a shows the measured device orientation from all 40 data collection test runs, and demonstrates that all subjects were able to safely perform the rapid extensions. Figure $3.9 \mathrm{~b}$ shows that the tracking error remained within the theoretical error bounds proposed in Theorem 1 at all times and for all users, and confirms that subject-specific effects cannot destabilize the position controller. These results also suggest that the impedance manipulation parameters do not need to be tuned for each individual. However, the device was observed to exhibit oscillations in some test runs. While these oscillations are undesirable, no test run exhibited a violent instability and no subject was compelled to depress the emergency switch due to the oscillations. Also, the observed oscillations did not have the potential to cause harm and could be subdued by subjects if they adjusted their joint stiffness.

Feedback from subjects indicated that these oscillations reflected the different strategies that they adopted when interacting with the device. An example of this can be seen in Figure 3.10 which presents results from one subject who repeated the experiment with and without a stiff elbow joint. As can be seen in the figure, stiffening the elbow joint at the end of the extension resulted in increased oscillations and a longer settling 
time. Keeping the elbow joint loose resulted in larger overshoot, but reduced oscillations overall. Accordingly, the results from this experiment show that subject-specific effects can contribute to oscillatory responses. However, with a little practice and coaching, users can learn specific strategies that help avoid oscillatory responses.

\subsubsection{Comparison with Different Interaction Controllers}

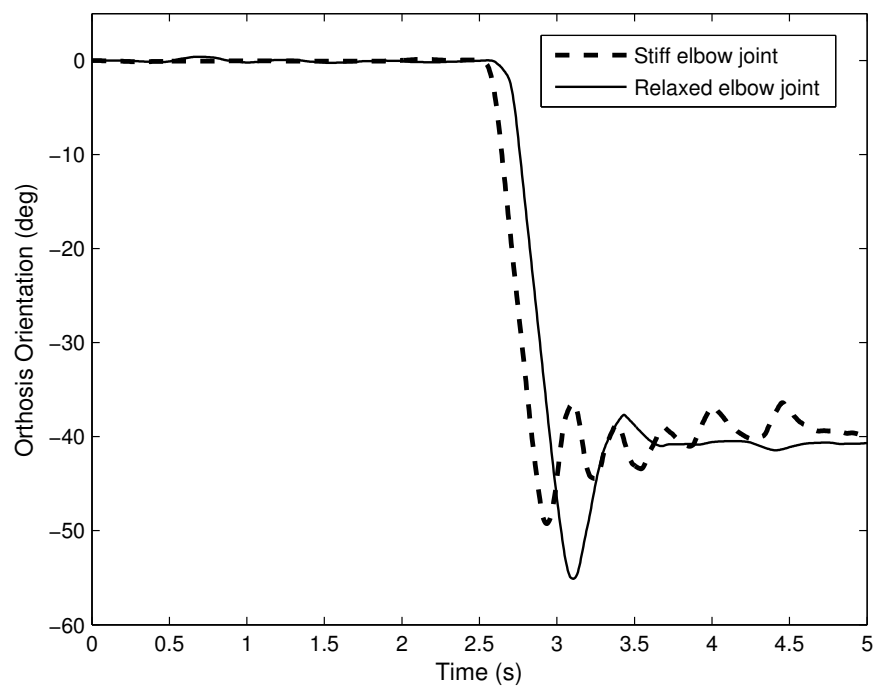

Figure 3.10: Oscillations caused by a stiff elbow joint. Larger oscillations were observed when the subject attempted to avoid overshoot by keeping his elbow joint stiff. Though the overshoot is larger, no sustained oscillations are observed when the user made an active effort to remain relaxed at the end of the extension.

A variety of other interaction controllers [124,127-129,132,133] and control loop design procedures $[118,119,121,126,149]$ exist in the literature. These interaction controllers and design procedures attempt to shape the actuator command - or provide systematic methods for selecting controller gains - to ensure that the interaction remains stable. The impedance manipulations proposed here are unique because they stabilize the position control loop by limiting the allowable motion of the haptic device. As such, these manipulations do not constitute a control law in the typical sense. They are best viewed as an added safety 
layer in the overall control structure, and could easily be combined within an admittance control structure that uses more sophisticated position controllers (e.g., $[127,132,133])$.

Generally speaking, interaction controllers based on robust control principles (e.g. $[110,117-121,128,130])$ require approximate dynamic models of the user and device, and in most cases, bounds on the uncertainties in these models. In contrast, the impedance manipulations can be implemented without modeling the user's or device's dynamics. Moreover, many of the aforementioned controller design methodologies [110, 117-121] translate into complicated optimization problems. These design procedures require defining an approximate model of the interaction, bounds on uncertain parameters, measures of stability and performance, and any relevant constraints. A subsequent search procedure must be used for finding parameter sets (e.g., feedback or feedforward controller gains) that optimize the stability and performance measures [110,117-121].

As evidenced in the corresponding literature [110,117-121], the structure and resolution of the interaction model, the degree of model information available, the design of the stability/performance measures, and the assumed structure of the controller can greatly influence the results obtained. Thus, there are endless possibilities and combinations, and implementing these design procedures requires a highly skilled designer. This approach is advantageous because it allows a designer to leverage the available information to design an optimal solution [119]. However, the robust stability measures proposed in these works are still conservative. Even if improved stability and performance measures such as those proposed in [119] are used, these design procedures may yield unnecessarily conservative controllers (e.g., compare results from competing methods in [120] versus [119]).

Interaction controllers based on adaptive control principles can help overcome the difficulties associated with modeling the user's and device's dynamics [129]. The adaptive controller proposed in [129], for example, accommodates uncertain nonlinear models of the haptic interface and an uncertain mass-spring-damper model of the user; careful 
design of the filter used for the measured interaction forces allows the controller to achieve apparent mass reductions as high as $89 \%$. In general, adaptive controllers allow improved performance because they are less conservative. However, they have several drawbacks such as lack of robustness to unmodeled dynamics, degraded performance or instability in the case of time-varying parameters, and excessively large control signals [150].

If the impedance manipulations are used, tracking error bounds and actuator saturation limits may be used to easily specify the acceleration limit parameters (see Remark 1 of Theorem 1). If a proportional controller is used to close the position loop, then the proportional gain can be increased iteratively to the point that the haptic device exhibits noticeable oscillations or chatter. Next, the value of $b$ can be tuned to attenuate these oscillations. Only the value of $b$ would need to be tuned thereafter if new reference dynamics are simulated. Thus, with the impedance manipulations, the emphasis shifts from careful design to careful tuning. And, as the experiment results in the previous sections demonstrate, the simple tuning procedure can yield impressive performance such as the ability to render apparent inertia reductions as high as $99.3 \%$.

The concept of modifying the output of the interaction model has previously been investigated. Beginning with the introduction of the time-domain passivity controller (TDPC) for haptics [151], many interaction controllers that selectively modify the target impedance to ensure passive interactions have been considered by researchers [122, 124, 152-155]. These controllers activate an energy dissipating element whenever a passivity violation is observed. The force-bounding algorithm presented in [154], for example, analogously defines the maximum and minimum force that the haptic device can generate at any instant to ensure a passive interaction. The passivity layer of the two-layer approach for bilateral manipulator systems [155], only applicable to impedance-controlled devices, analogously presents time-varying bounds on the actuator torques. Similarly, the adjusting output limiter in [122] imposes a limit on the maximum force generated from the reference dynamics to ensure passivity. 
All these controllers are simple to implement, model-free, and can provide equally provide stable interactions. However, the methods for modifying the target impedance they encompass are based on the passivity formalism. As such, they are naturally more conservative than the impedance manipulations which are based on a less stringent condition of stability in the sense of UUB, and arguably, more closely aligned with concept of semi-passivity proposed in [156]. Moreover, these controllers are typically used when simulating virtual walls with impedance-controlled devices. As such, they may be less effective in simulating free motion in admittance-controlled haptic devices because they do not explicitly account for the instability/energy generated by the position controller.

Also, the stability properties of many interaction controllers are dependent on an implicit assumption that the haptic device's actuators can deliver the forces/torques requested by the control law $[127,129,132,133]$. Thus, many robust and adaptive controllers designed using Lyapunov stability arguments, could be destabilized whenever a haptic device' actuators are incapable of manifesting the desired control action. The acceleration limits are inherently more robust in these situations because they treat the reference acceleration as modifiable input whose action can supplement the action of the control law. In other words, when the actuators or control law are incapable of tracking a highly dynamic motion, the acceleration limits the modify the commanded motion of the device to ensure that the position control loop remains stable.

\subsection{Extension to Position Control Applications}

The discussions, so far, have focused on using the impedance manipulations to achieve stable interactions in admittance-controlled haptic devices. However, the impedance manipulations can equally be used in any generic position control problem. This section briefly demonstrates how, and presents experimental results that confirm that the impedance 
manipulations can outperform conventional position controllers.

The discussion in this section does not apply to the problem of human-robot interaction control. It is presented here only because it describes an extension of the control methodology described in Sections 3.2 and 3.3. This generic position control methodology could be used, for example, for commanding GaitEnable to follow a pre-defined trajectory.

\subsubsection{Overview}

Outer Loop

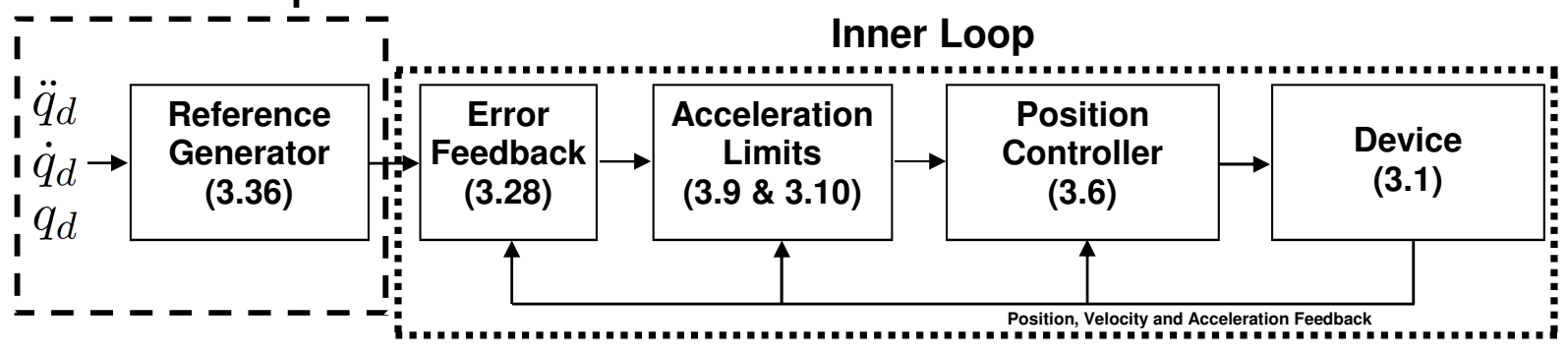

Figure 3.11: A block diagram of the dual-loop position controller. The impedance manipulations guarantee bounded tracking errors in the inner loop. The user's dynamics can be neglected since this control structure is designed for position control applications.

In a typical position control problem, one attempts to design a control law that guarantees: i) stability, ii) accurate tracking of a desired trajectory, and iii) robustness to confounding factors such as disturbances, model uncertainties, and actuator saturation. The impendence manipulations can be used to build a stable and robust position controller that satisfies all these requirements if the reference model is defined as follows:

$$
\Gamma_{1}\left(\ddot{q}_{d}-\ddot{q}_{r}\right)+\Gamma_{2}\left(\dot{q}_{d}-\dot{q}_{r}\right)+\Gamma_{3}\left(q_{d}-q_{r}\right)=0
$$

where $\Gamma_{1}, \Gamma_{2}$, and $\Gamma_{3}$ are diagonal matrices, $q_{d}$ is the desired trajectory, and $q_{r}$ denotes the reference trajectory. It is important to note that $q_{d}$ defines the nominal trajectory that the 
robot is expected to follow, while $q_{r}$, as defined before, denotes the final commanded motion of the robot that the position controller tracks. If $\Gamma_{1}, \Gamma_{2}$, and $\Gamma_{3}$ are positive-definite, then $q_{r} \rightarrow q_{d}$ as $t \rightarrow \infty$. Furthermore, if a position controller ensures perfect tracking of $q_{r}$ (i.e., if $q=q_{r}$ ), then it follows that $q \rightarrow q_{d}$ as $t \rightarrow \infty$.

The proposed position control architecture is shown in Figure 3.11 and comprises a dual-loop position control structure. The outer loop, whose dynamics are governed by (3.36), drives the reference trajectory $q_{r}$ to the desired trajectory $q_{d}$. The inner loop, a position control loop stabilized by the impedance manipulations, drives the robot's trajectory $q$ to the reference trajectory $q_{r}$. As a result, the position tracking error $q_{d}-q$ is the sum of the outer loop tracking error $q_{d}-q_{r}$ and the inner loop tracking error $q_{r}-q$.

The diagonal coefficients of $\Gamma_{1}, \Gamma_{2}$, and $\Gamma_{3}$ determine how quickly and smoothly $q_{r}$ approaches $q_{d}$. Standard criteria such as the desired rise-time, overshoot, and/or natural frequency may be used for selecting $\Gamma_{1}, \Gamma_{2}$, and $\Gamma_{3}$.

More concretely, using the dual-loop control structure entails the following at each time step:

1. Isolating and calculating $\ddot{q}_{r, i}$ from (3.36).

2. Applying error feedback, if needed, using (3.28). The value of $b$ can be tuned to attenuate chatter.

3. Using (3.9) and (3.10) to limit the reference acceleration.

4. Integrating the result of the previous step to generate the reference trajectory $q_{r, i}$.

5. Implementing a position controller to track the reference trajectory $q_{r, i}$. 
Different types position controllers may be used in Step 5. Using a P or PD position controller is advantageous, however, as it results in a model-free position control.

\subsubsection{Tracking Error Bounds}

As the inner loop is stabilized by the impedance manipulations, Theorem 1 in Section 3.2.2 can be used to show the stability of the inner position control loop. It also follows from this theorem that,

$$
\left|q_{r, i}-q_{i}\right| \leq \frac{\eta}{\gamma \phi} \quad \text { if } t>>t_{o}
$$

In the event that the outer loop exactly obeys (3.36), then $q_{d}-q_{r} \rightarrow 0$ as $t \rightarrow \infty$. Using (3.37), it can be shown that the total position tracking error $q_{d, i}-q_{i}=\left(q_{d, i}-q_{r, i}\right)+\left(q_{r, i}-q_{i}\right)$ must also be bounded, i.e., that,

$$
\begin{aligned}
\left|q_{d, i}-q_{i}\right| & \leq\left|q_{d, i}-q_{r, i}\right|+\left|q_{r, i}-q_{i}\right| \\
& \leq \frac{\eta}{\gamma \phi} \text { if } t>>t_{o} .
\end{aligned}
$$

As noted previously, the stability of the outer loop is easy to confirm if $\Gamma_{1}, \Gamma_{2}$, and $\Gamma_{3}$ are selected to be positive-definite. However, the assumption that $q_{r}-q_{d} \rightarrow 0$ as $t \rightarrow \infty$ does not hold if error feedback is applied during Step 2 or if the reference acceleration is limited during Step 3. This is because the impedance manipulations (3.28), (3.9) and (3.10) act as perturbations that stop the outer loop (3.36) from driving $q_{r}$ to $q_{d}$. Thus, (3.38) only gives a measure of the best possible performance that can be achieved. If the desired trajectory $q_{d}$ is unreachable (e.g., if an obstacle restricts the robot's motion, or 
if the actuators cannot generate enough force to follow a dynamic trajectory), then the maximum tracking error may be larger than (3.38).

The fact that the impedance perturbations disturb the outer loop dynamics is not necessarily a disadvantage. In the interaction control scenario, the impedance manipulations modify the reference acceleration, when necessary, to ensure a stable human-robot interaction. In the dual-loop position control structure, the impedance manipulations analogously command the robot to deviate from $q_{d}$ when factors such as disturbances or saturation make it difficult for the robot to follow $q_{d}$. However, as predicting the maximum tracking error during operation is challenging, iterative tuning of the inner and outer loop parameters may be necessary to satisfy a particular tracking error specification.

\subsubsection{Comparing the Dual-Loop Controller to a Conventional PD Con- troller}

Several experiments were performed to investigate how a PD controller with impedance manipulations compares to a conventional PD controller. The single DOF robotic device described in Section 3.4.1 was used for these experiments.

Both step response and sinusoid tracking experiments were performed at the following test conditions:

1. PD - A conventional PD controller used to track the desired step or sinusoidal trajectory $q_{d}$.

2. $\mathbf{P D}+\mathbf{A L}-$ The proposed dual-loop control structure with (3.36) used to calculate the reference acceleration $\ddot{q}_{r}$ and (3.28), (3.9) and (3.10) used to stabilize a PD controller that tracks $q_{r}$. 


\section{Controller Parameter Selection}

The impedance manipulation and reference model parameters were selected as: $\psi=25 \frac{\mathrm{rad}}{\mathrm{s}^{2}}$, $\gamma=500 \frac{1}{s}, \eta=600 \frac{\mathrm{rad}}{\mathrm{s}^{2}}, b=167, \Gamma_{1}=1, \Gamma_{2}=2000$, and $\Gamma_{3}=200000$. A first-order, unity gain low-pass filter with a bandwidth of $12 \mathrm{rad} / \mathrm{s}$ was used to filter $\dot{s}$ before (3.28) was evaluated.

$\mathrm{P}$ and $\mathrm{D}$ gains of $30000 \frac{\mathrm{Nm}}{\mathrm{rad}}$ and $150 \frac{\mathrm{Nm} \cdot \mathrm{s}}{\mathrm{rad}}$ were used at the PD test condition. $\mathrm{P}$ and

D gains and $80000 \frac{\mathrm{Nm}}{\mathrm{rad}}$ and $400 \frac{\mathrm{Nm} \cdot \mathrm{s}}{\mathrm{rad}}$ were used at the PD + AL test condition. The gains used at the PD test condition reflected the largest proportional and derivative gains that could be used before the position controller exhibited chattering. When the impedance manipulations were active (i.e., at the $\mathrm{PD}+\mathrm{AL}$ test condition), proportional and derivative gains nearly 2.7 times larger could be used before any chattering was observed.

\section{Step Response Results}

The device was commanded to track a 30 degree step from a neutral position, hold position, and then track a 60 degree step in the opposite direction. Six data collection test runs were performed at each test condition to ensure the repeatability of the results. Results from all 12 test runs can be seen in Figure 3.12.

Figure $3.12 \mathrm{a}$ shows that the step responses are qualitatively similar both test conditions. However, a zoomed-in view of the tracking error in Figure 3.12b reveals that the settling time was reduced by $0.25 \mathrm{~s}$ at $\mathrm{PD}+\mathrm{AL}$ test condition. The initial rise to the set point was similar at both test conditions as the motor's acceleration was restricted by the current limit of the servo drive. Figure $3.12 \mathrm{~b}$ also shows that the tracking performance was repeatable at both test conditions.

Further analysis also revealed that the average RMS steady-state tracking error at 


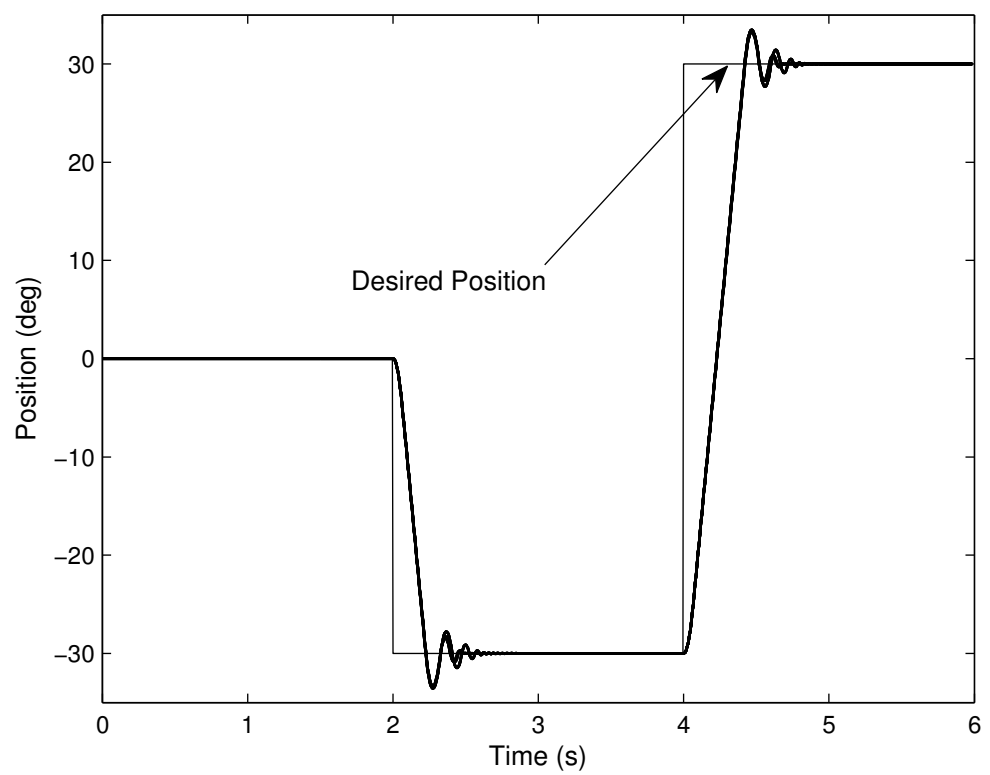

(a)

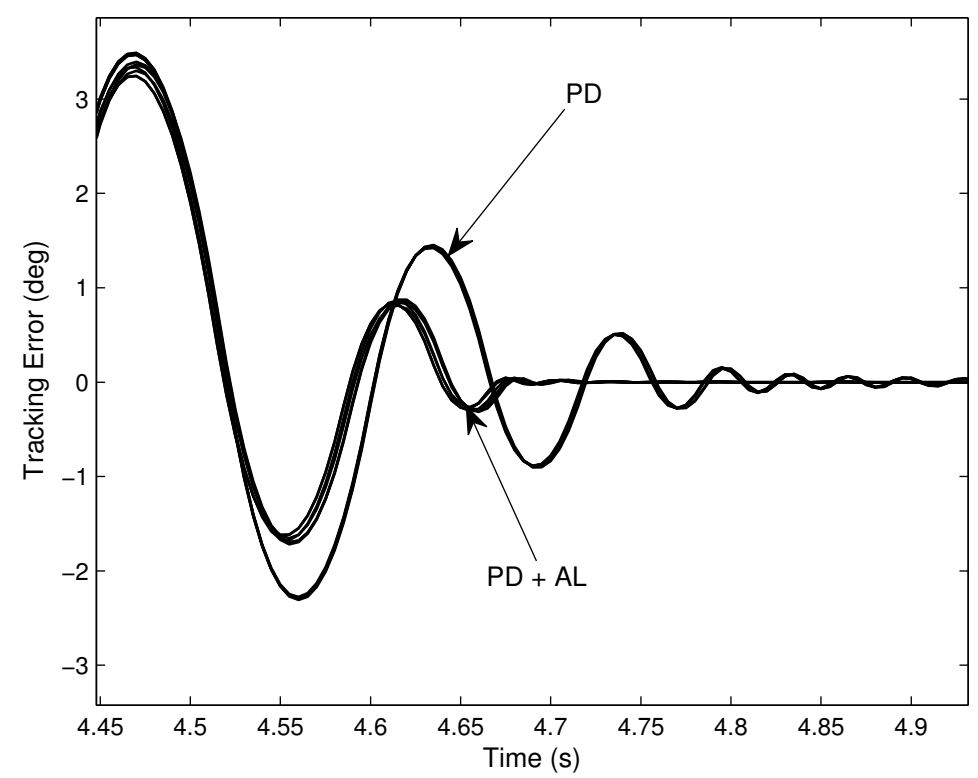

(b)

Figure 3.12: Improved tracking performance during step responses. (a) Orientation. (b) Tracking error. The proposed dual-loop control structure (PD + AL test condition) results in a reduced steady-state error and setting time. 
$\mathrm{PD}$ test condition was 0.0020 degrees. This average was taken over both the 30 and 60 degree steps in all six data collection test runs; a total of 12 samples of RMS steady state error were collected and averaged at the PD test condition). The equivalent average RMS steady-state error at the PD + AL test condition, 0.00096 degrees, was less than half the value observed at the PD test condition. Thus, both the settling time and steady-state error results confirm that the dual-loop control structure outperformed the PD controller. This result is significant because it demonstrates that the impedance manipulations can achieve a significant performance improvement without the need to implement a sophisticated position controller in the inner loop. As simple linear controllers such as PD controllers are still used in a majority of applications, the impedance manipulations offer a simple addition that can enhance performance and guarantee stability.

The fact that higher $\mathrm{P}$ and $\mathrm{D}$ gains could be used at the PD $+\mathrm{AL}$ test condition explains why better performance was achieved with the dual-loop position controller. To demonstrate what happens when the impedance manipulations are removed, the experiment was repeated with the gains of the conventional PD controller increased to the values to $80000 \frac{\mathrm{Nm}}{\mathrm{rad}}$ and $400 \frac{\mathrm{Nm} \cdot \mathrm{s}}{\mathrm{rad}}$. Figure 3.13 shows a close-up of the device orientation as it settles to the -30 degree set point. As seen in the figure, the PD controller without impedance manipulations exhibits a longer settling time, and sustained oscillations with a finite frequency and amplitude that are characteristic of the chattering phenomenon. No chattering was observed when the impedance manipulations were used (i.e., at the PD + AL test condition). Thus, these results confirm the conjecture in Remark 2 of Theorem 1 that the impedance manipulations allow the use of significantly larger control gains.

\section{Dynamic Tracking}

Additional experiments were performed to confirm that the dual-loop controller can be used to track dynamic trajectories. The experiments were repeated at the $\mathrm{PD}$ and $\mathrm{PD}+\mathrm{AL}$ test condition with relevant controller parameters selected as noted in Section 3.5.3. The 


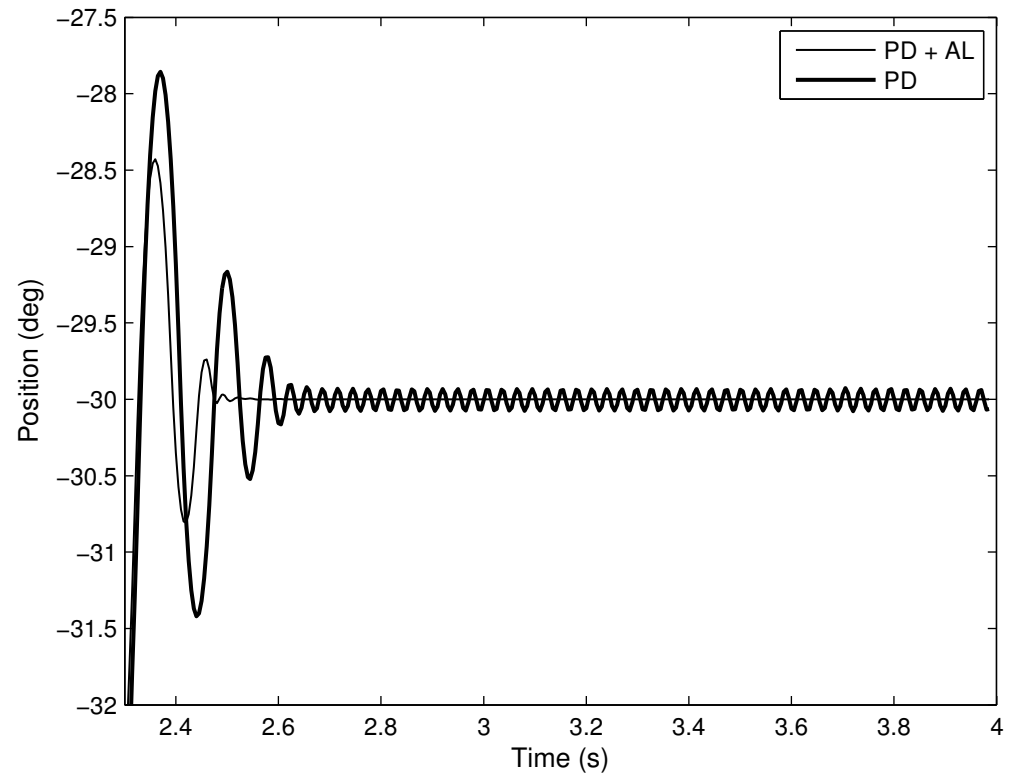

Figure 3.13: Encountering the chattering phenomenon at high position controller gains. A convention PD controller with high gains exhibits sustained oscillations with a finite frequency and amplitude. There is no chattering observed when the same gains are used with the dual-loop position control structure. 
desired trajectory, a sinusoid with an amplitude of $1.25 \mathrm{~Hz}$ and amplitude of 30 degrees, was the fastest trajectory that the device could track (assuming a 30 degree amplitude) before actuator saturation effects significantly interfered with tracking accuracy. In the interest of removing actuator saturation as a complicating factor, faster trajectories were not considered. Results from the experiment are shown in Figure 3.14.

Figure $3.14 \mathrm{a}$ shows that the transient responses of the device between 0 and 0.5 seconds are almost identical for both the $\mathrm{PD}$ and $\mathrm{PD}+\mathrm{AL}$ test conditions. This result demonstrates that the outer loop does not interfere with the transient response. The figure also suggests that both the conventional PD controller and the dual controller appear to be equally effective at tracking the desired trajectory. However, a zoomed-in view of the tracking error in Figure 3.14b clearly shows that the dual-loop controller exhibits better tracking performance. The RMS tracking error at the PD + AL test condition, 0.0119 deg, was nearly 2 times smaller than the value calculated at the PD test condition (0.0203 deg). This result confirms that the improved performance of the dual-loop controller extends to dynamically varying trajectories as well.

\section{Comparing the Dual-Loop Structure with Alternative Controllers}

Countless different types of linear and nonlinear position controllers have previously been investigated by other researchers [157]. The discussion below will focuses on comparing conventional $\mathrm{PD} / \mathrm{PID}$ controllers to the dual-loop structure, as PD/PID controller are used most frequently in practice [158], and because the dual-loop controller could easily replace conventional $\mathrm{PD} / \mathrm{PID}$ controllers in many applications. While simple to implement, $\mathrm{PD} / \mathrm{PID}$ controllers can be difficult to tune and do not guarantee stability unless an appropriate gain selection procedure is used [158].

Numerous examples of such gain selection procedures can be found in the literature (see [132, 158-161] and references therein). For rigid manipulators with dynamics (3.2) 


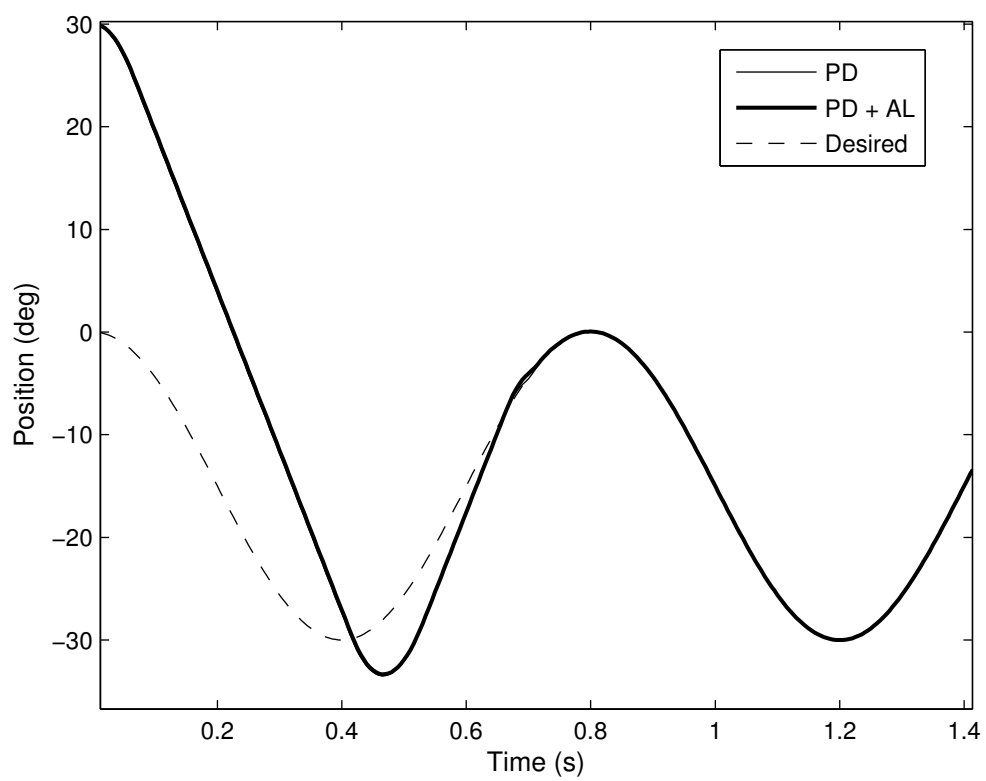

(a)

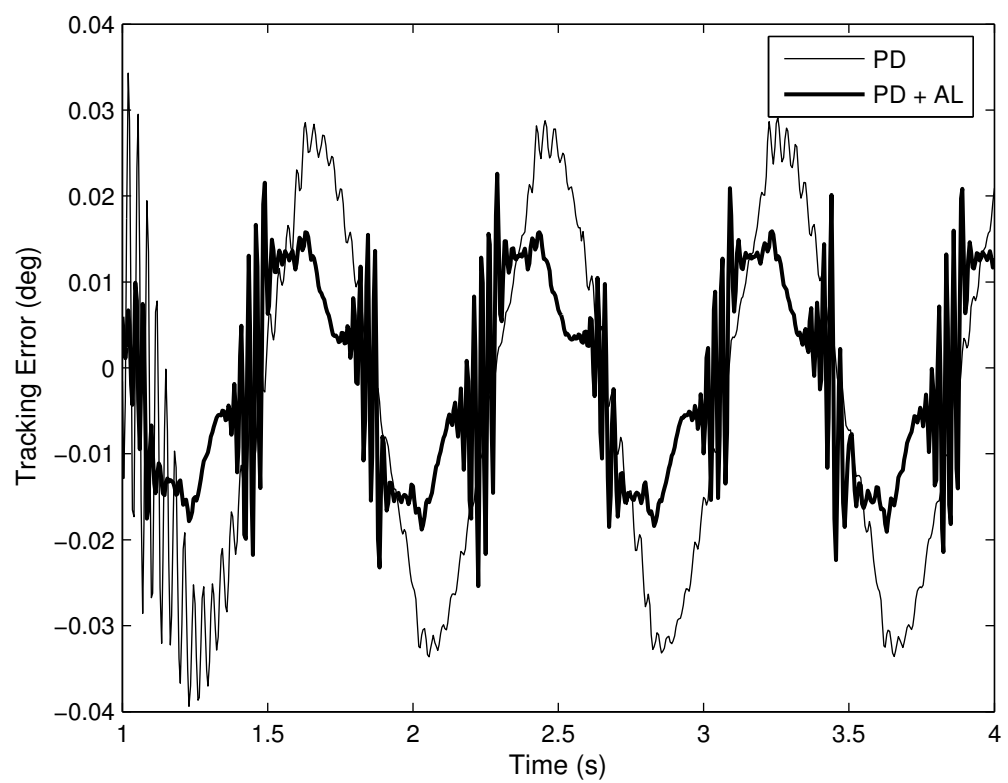

(b)

Figure 3.14: Improved tracking performance during dynamic trajectory tracking. (a) Orientation. (b) Tracking error. The dual-loop controller, denoted as the PD + AL test condition in the figure, reduces the RMS tracking error tracking by nearly $50 \%$. The results also demonstrate that the dual-loop controller can be used for time-varying trajectories. 
or (3.1), gain selection procedures for PD/PID controllers are typically derived by searching for Lyapunov functions or inequality conditions that guarantee the stability of the error dynamics. However, these procedures and inequality conditions can be quite complex or require that the desired trajectory vary slowly [158]. Using the impedance manipulations (3.28), (3.9) and (3.10) to stabilize the inner loop provides a simpler alternative.

Moreover, many of the aforementioned gain selection procedures require a priori knowledge of disturbance bounds and manipulator properties such as the maximum singular values of the mass matrix. In contrast, mass, damping, coriolis, centrifugal, and gravity terms are purposely excluded in the stability proofs in Sections 3.2 and 3.2.3. This allows the impedance manipulations to stabilize the interaction without consideration of the specific characteristics of the manipulator.

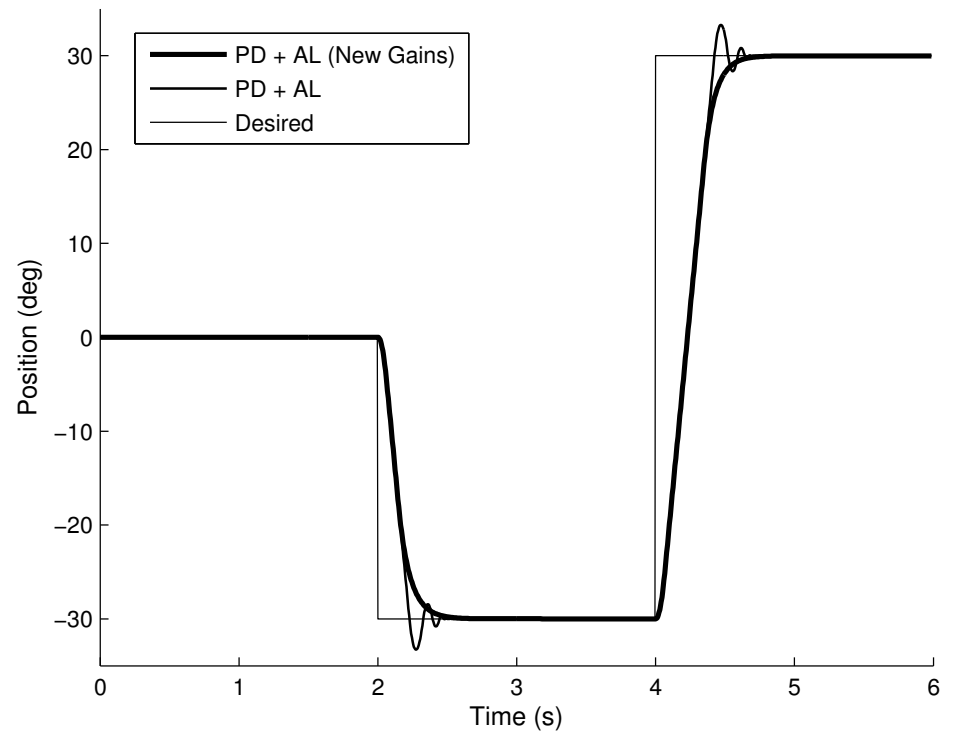

Figure 3.15: Tuning the outer loop to improve the transient response. The outer loop parameters can be tuned - independently of the position control gains used in the inner loop - to achieve a damped response without overshoot. 
Alternative control methods that account for actuator input constraints and saturation can pose even further implementation challenges. Anti-windup controllers for saturation need mechanisms for resolving the inconsistency between the internal controller states and the system states $[134,135]$ and reference governors that reshape a desired trajectory to meet state and input constraints require solving an optimization problem in real-time $[145,146]$. Some forms of adaptive-backstepping [147] and model-predictive control [148] can also accommodate input constraints but are similarly difficult to implement. The impedance manipulations are simple to implement, and have already been demonstrated to ensure bounded tracking errors under actuator saturation, as in the results in Section 3.4.4 for example. Allowing $q_{r}$ to deviate from $q_{d}$ via the influence of the impedance manipulations, provides a way for addressing the inconsistency between the internal controller states and the system states that arise under actuator saturation.

Finally, in contrast to conventional PD or PID controllers, the dual-loop position control structure also affords a greater degree of flexility in tuning the transient response. To demonstrate this, the experiment was repeated at the PD $+\mathrm{AL}$ test condition with inner loop parameter held constant, and outer loop parameters set to $\Gamma_{1}=1, \Gamma_{2}=2500$, $\Gamma_{3}=2500$. The results, shown in Figure 3.15, demonstrate that the outer loop parameters were effective in eliminating overshoot, and attenuating oscillations about the set point.

With a PD or PID controller, the transient response can only be tuned by manipulating the proportional and derivative gains. As sensor noise in the velocity estimate limits the maximum value of the derivative gain, designers may be forced to use a lower proportional gain to achieve a well damped response. This, in turn, results in poorer disturbance rejection. The dual-loop structure helps overcome this limitation. The impedance manipulations in the inner loop allow the use of higher position control gains and enable the position controller to remain robust to disturbances. Once the desired inner loop parameters are selected, the outer loop parameters can be further tuned to achieve the desired transient response. 


\subsection{Chapter Summary}

This chapter introduced a novel stabilizing controller for admittance-controlled haptic devices. The enhanced control combines a typical admittance control loop with a set of impedance manipulations that intermittently modify the commanded motion of the device to ensure stable interactions in coupled human-robot systems. The first impedance manipulation, a dynamically-varying limit on the commanded motion of a haptic device, ensures the stability of the device's position control loop. The second manipulation, tracking error feedback in the reference acceleration, selectively attenuates sustained oscillations in the haptic device's response.

The enhanced controller differs from typical interaction controllers because it is not model-based, and results in a stable, model-free control if a model-free position controller is used to track the commanded motion of the robot. A Lyapunov stability analysis demonstrates that the first impedance manipulation, the reference acceleration limits, guarantee bounded tracking errors. The second impedance manipulation, which requires reshaping the reference acceleration with error feedback, is the necessary element that facilitates the display of small impedances; error feedback in the reference model inhibits the tracking error from diverging too quickly, and minimizes the degree of instability that the device's position control loop exhibits when displaying a small mass. As a whole, the enhanced controller, derived for both joint-space and task-space admittance control, results in simple-to-implement admittance control with guaranteed stability, and the unique ability to display a small apparent mass.

Experiments performed with a simple 1-DOF haptic device demonstrate that the impedance manipulations allow: i) stable interactions under a wide range position controller gains, ii) stable interactions under actuator saturation, iii) apparent inertia 
reductions as high as $99.3 \%$, iv) stable interactions during highly dynamic motions, v) predictable changes in the haptic device's response when the reference model parameters are varied, and vi), robustness to subject-specific effects. All these features make them well-suited for use with wearable robotic systems for rehabilitation where displaying small apparent inertias is critical. Results from experiments also confirm that the measured performance of the haptic device is consistent with the analysis - the tracking error bounds proposed in Theorem 1 are obeyed even at extreme test conditions such as a $99.995 \%$ inertia reduction, and error feedback in the reference model minimizes the RMS value $\dot{V}$.

Other interesting results, such as the emergence of oscillatory responses associated with the strategies that user's adopt when interacting with the haptic device, were also encountered. Experimental observations suggest that a user's (actively controlled) joint stiffness can contribute to oscillatory interactions, but also that user's can train themselves to remain relaxed and avoid such oscillatory interactions. Other results also demonstrate that actuator saturation can lead to instability, and these instabilities are insidious and input-dependent. Interestingly, saturation effects did not cause instability in the position tracking experiments. This result is noteworthy because it highlights that saturation-instigated instabilities are problematic as a result of coupling of the human, robot, position controller, and reference model dynamics.

The chapter concluded with a short section demonstrating the application of the impedance manipulation to generic position control problems, and introduce a novel dualloop position control architecture stabilized by the impedance manipulations. The outer loop generates an intermediate motion command that drives a robot towards a nominal trajectory. The inner position control loop, stabilized by the impedance manipulations, drives the robot towards the intermediate motion command. The dual-loop controller is simple to implement yet very powerful. Its advantages include: i) guaranteed stability, ii) the ability to accommodate actuator saturation, iii) improved disturbance rejection via the use of larger position control gains, iv) independent control over the transient response, 
and v), a model-free implementation when coupled with a model-free position controller.

It is also important to highlight that the experiments described here were performed with a simple haptic device. Nonlinear dynamics, coupling effects, and other confounding factors that arise in more complicated system were not considered. However, Chapters 4 and 5 present results from numerous experiments performed with a 3-DOF mobile gait trainer with coupling effects and nonlinear wheel-ground dynamics, that mirror many of the key results shown in this chapter. Moreover, gauging from numerous times that an unstable interaction was observed with this simple 1-DOF device, it is clear that the results in this chapter are still highly relevant for 1-DOF wearable robots such as knee, elbow, or ankle orthoses that pose a safety risk for their users. 


\section{Chapter 4}

\section{GaitEnable: A Robotic Gait Trainer for Overground Gait Training}

GaitEnable, a robotic device designed to facilitate overground gait training, is introduced in this chapter. The chapter begins with a review of the challenges that motivated the design of the system, and a brief discussion about the major design assumptions and choices. It also provides details about key subsystems, and presents results from experiments performed to validate the stability of GaitEnable's interaction control system.

\subsection{Introduction}

Focused and repetitive gait training in functional environments and contexts is essential during gait rehabilitation therapy. Such therapy has been proven to encourage recovery and enables patients with physical impairments to regain their mobility and independence [4]. However, delivery of this care is physically demanding, and unsafe in many circumstances, as caregivers must take on the burden of lifting, moving, and supporting individuals who may have little capacity to stand and maintain their balance. These challenges have prompted significant recent research towards the design of robotic of gait rehabilitation devices for automating physical therapy $[2,15,18,19,21,72,73,162]$.

As discussed in Chapter 2, robotic gait rehabilitation devices may be separated into 
three different categories depending on their function and design: $i$ ) end-effector-type robots like the Haptic Walker [15] that attach to the user's feet and guide the motion of the user's feet $[15,18]$; $i i)$ exoskeleton-type robots like the LOPES [21] that attach parallel to the patient's lower extremities and assist the patient during treadmill walking [19,21]; and $i i i)$, mobile gait trainers with actuated mobile bases like the KineAssist [2] that enable overground gait practice $[72,73,162]$.

End-effector-type and exoskeleton-type robots are effective at reducing therapist workload and enable patients to safely practice stepping motions. However, they are less effective for balance training because they restrict patients to walking in place or over a treadmill, and limit training to the sagittal plane [51]. As task specificity is crucial to optimizing functional outcomes [24], mobile gait trainers that provide realistic overground gait practice may be more effective than these robots $[2,44]$. Thus, from the onset of the research, it was deemed essential that GaitEnable be capable of facilitating overground gait practice.

The constraints that a rehabilitation robot imposes on a user's pelvis and torso motion can limit the type and quality of training that a patient can receive. A variety of research already points to the detrimental effects that pelvis and torso motion constraints can impose during gait training $[47,48,51]$. It is worth nothing, however, that most robotic gait trainers $[72,73,162]$ are designed with non-holonomic differential steering systems that constrain a patient's lateral pelvis translation. Moreover, these devices cannot be used for important balance training activities such as side-stepping or one-leg balance. As such, they may not provide for a realistic practice of overground walking either. Thus, the development of GaitEnable was motivated by the desire of building a simple robotic gait trainer that imposes minimal motion constraints, enables balance training, and facilitates natural overground gait.

Other gait trainers like Lee and Lee's gait trainer [82], the Nanyang university 
platform [163], the Where I and Where II robots [72], and the WalkTrainer [73] include rotary encoders or sliding linear potentiometers in the patient constraint mechanisms that provide information about the user's walking speed and direction. This information is then used to generate a motion command for the mobile base that ensures that the device shadows the user's voluntary motion. Thus these control systems do not provide a direct method for transmitting specific force cues, and do not attempt to modify or influence the user's behaviour through an interaction control system. The KineAssist, in contrast, implements an "adaptive haptic algorithm." However, few details are provided beyond statements from the authors that a combination of force and velocity measurements from force and position sensors embedded within the device's pelvis harness are used to command the device's motion $[2,2]$. Thus, this research also sought to investigate whether an interaction control system driven purely by pelvis force inputs could allow individuals to command the motion of GaitEnable. A pelvic-driven interaction control system is desirable because it allows a BWS system to provide support from below as the BMT does. Support from below may by superior to using a torso harness as it allows users to practice the protective trunk postural reactions necessary to prevent falls [164].

\subsubsection{Training and Design Requirements}

The requirements discussed in Section 2.2 were also used to direct the conceptual design of GaitEnable. They are summarized in the list below:

- GaitEnable should reduce the therapist's workload.

- GaitEnable should provide body weight support and fall protection.

- GaitEnable should allow functionally-relevant training such as overground walking, side-stepping and lateral motions, and change-in-support reactions for balance training.

- GaitEnable should impose minimal constraints on the user's pelvis and torso motions. 
- GaitEnable should not prohibit manual manipulation of the patient's legs.

- GaitEnable should require a short setup time.

- GaitEnable should be easy for therapists and patients to use.

- GaitEnable's control system should automatically synchronize the device's motion with the patient's motion.

- GaitEnable's control system should be capable of detecting and preventing falls.

- GaitEnable's control system should be capable of providing force and motion cues consistent with the therapy goals.

\subsubsection{Limitations of the Bungee Mobility Trainer}

As noted in Chapter 2, the Bungee Mobility Trainer (BMT) consists of a passive mobile base and a 3 DOF passive linkage that supports the patient's pelvis from below; an image of the device can be seen in Figure 4.1. The BMT is a simple and practical mobile BWS system that has numerous beneficial features such as:

1. A 3 DOF passive linkage that provides graduated body-weight support and fall protection.

2. No constraints on leg and ankle motion, and minimal constraints on torso, pelvis and hip motion in comparison to many other robotic devices $[2,15,18,19,21,72,73,162]$.

3. A simple design and short setup time.

However, the BMT is a passive device, and like all similar passive devices, it has the following disadvantages:

1. It cannot provide the propulsion forces required to initiate, sustain, or restrict the patient's gait. As such, it cannot provide force and motion cues consistent with the therapy goals or automatically detect and prevent falls. 


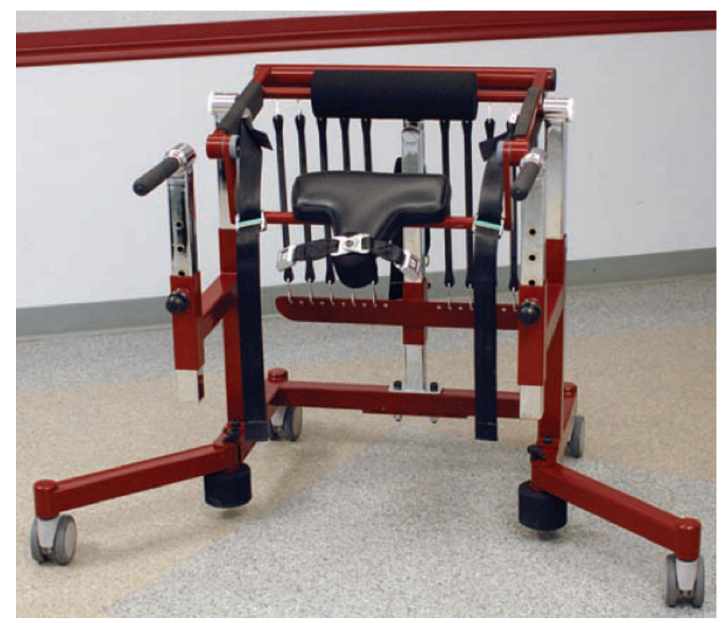

Figure 4.1: An image of the Bungee Mobility Trainer.

2. It cannot be programmed to emulate different types of behaviors (e.g., gait stabilization, assistance, resistance, disturbance generation, etc.). Thus, it cannot easily be used for targeted training activities such as pure side-stepping.

3. It cannot actively perturb or stabilize the patient during balance training. Thus, it cannot be used to implement targeted, perturbation-based balance training programs such as those described in $[57,165-167]$.

4. It is not equipped with sensors that can be used for tracking gait characteristics. Thus, additional measurement equipment must be used during the therapy session to track key gait characteristics such as gait speed.

5. Its mass and inertia can limit frail patients from easily walking in the device. Having to push/pull a large mass imposes alters the user's natural pelvis and torso motions.

6. Its mobile base exhibits singularities at certain configurations. Motions such as pure rotation can be difficult to initiate when the casters are in a singular configuration. Accordingly, the caster-based mobile base periodically inhibits natural pelvis and torso motion. 
As the BMT's BWS system helps satisfy many of the aforementioned design guidelines, the first prototype of GaitEnable capitalized on the benefits of the BMT's BWS system and introduced targeted robotic elements to help overcome its limitations.

\subsection{Overview of the GaitEnable Gait Trainer}

GaitEnable modifies the mechanical and mobility design of the BMT, and adds robotic control to achieve the key training and design requirements goals. The actuated mobile base allows users to manipulate the device with less effort, and is capable of assisting with stepping motions. The actuated mobile base also allows the user to translate laterally and rotate. This reduces motion constraints, facilitates a more natural gait, and enables the user to perform change-in-support reactions and other balance training activities that are difficult to perform with exoskeleton- and end-effector-type rehabilitation robots. Moreover, GaitEnable's interaction control system automatically synchronizes the device's motion with the user's voluntary motion and is capable of providing force cues for assisting or perturbing the user's motion. GaitEnable also has a short set-up time, and allows the therapists to manipulate the patient's legs.

The first prototype shown in Figure 4.3 is composed of the BMT's passive BWS system and frame, an omniwheel-actuated mobile base, and an interaction control system that allows the device to assist the user's voluntary motion. This prototype was designed for validating the performance of the new mobility and interaction control systems. Accordingly, features such as control interfaces for the therapist, and actuated BWS systems were not considered for this prototype. The requirement for making sure the device is easy for therapists to use, and studies to investigate whether GaitEnable can reduce therapist workload were not considered in detail.

Figure 4.2 summarizes the device requirements proposed in Section 2.2, and shows how GaitEnable compares with respect to other types of rehabilitation robots. As can 


\begin{tabular}{|c|c|c|c|c|}
\hline & End-effector-type robot & Exoskeleton-type robot & $\begin{array}{c}\text { Actuated mobile gait trainer } \\
\text { without omnidirectional mo- } \\
\text { bile bases }\end{array}$ & GaitEnable \\
\hline Provides body weight support & $\mathrm{x}$ & $\mathrm{x}$ & $\mathrm{x}$ & $\mathrm{x}$ \\
\hline Minimizes therapist workload & $\mathrm{x}$ & $\mathrm{x}$ & $\mathrm{x}$ & $\mathrm{x}$ \\
\hline $\begin{array}{l}\text { Provides an automated means for } \\
\text { supporting swing leg motion }\end{array}$ & $\mathrm{x}$ & $x$ & $\mathrm{x}$ & \\
\hline $\begin{array}{l}\text { Easily adapts to different body mor- } \\
\text { phologies }\end{array}$ & $\mathrm{x}$ & & $\mathrm{x}$ & $\mathrm{x}$ \\
\hline Easy for therapist to use & $x$ & & $\mathrm{x}$ & $\mathrm{x}$ \\
\hline Emulates overgound walking & & & $x$ & $x$ \\
\hline Imposes minimal motion constraints & & & & $\mathrm{x}$ \\
\hline Facilitates targeted balance training & & & & $\mathrm{x}$ \\
\hline $\begin{array}{l}\text { Allows practice of change-in-support } \\
\text { reactions }\end{array}$ & & & & $x$ \\
\hline Allows turning while walking & & & & $\mathrm{x}$ \\
\hline $\begin{array}{l}\text { Allows therapist to manually ma- } \\
\text { nipulate patient's legs }\end{array}$ & & & $\mathrm{x}$ & $\mathrm{x}$ \\
\hline Requires a minimal setup time & & & $\mathrm{x}$ & $\mathrm{x}$ \\
\hline Low cost & & & $\mathrm{x}$ & $\mathrm{x}$ \\
\hline
\end{tabular}

Figure 4.2: Comparing GaitEnable to different types of rehabilitation robots with respect to the requirements defined in Chapter 2. The letter $\mathrm{X}$ denotes that the robot-type has the desired quality or capability.

be seen from the figure, GaitEnable's primary benefit is its ability to allow training of a wider range of activities. This improved training capability is a result of GaitEnable's omnidirectional mobile base which provides users the freedom to move more naturally.

GaitEnable may be beneficial for any patient that requires assistance from two or more caregivers to mobilize. Stroke and bariatric patients, two patient populations that require significant physical assistance during rehabilitation therapy, are likely to benefit most from the use of this device. However, as the focus of this research was to validate the GaitEnable's mobility control system, only healthy subjects were recruited for experiments.

The process of using GaitEnable during therapy session can be summarized as follows:

1. The user tightens a belt around their waist that attaches them to the device.

2. The researcher specifies control system parameters such as the desired mass or assistance force that GaitEnable should display, and enables the control system from a 
nearby computer.

3. The user walks in their desired direction, and the device follows, assists, and/or perturbs the user's motion as per the controller settings and experiment requirements.

4. The researcher disables the control system and stops the device when the experiment is finished. The user can also depress a switch to disable the motors during an emergency.

No joystick, or other similar interfaces are required to control the motion of the device. The forces measured at the user-device interface when the patient pushes against GaitEnable, are translated into a motion command for GaitEnable's mobile base. This motion command is then transformed into corresponding desired omniwheel trajectories, and position controllers implemented at each motor-powered omniwheel track these trajectories. With this, users control when GaitEnable's motion is initiated, how quickly it moves, and the direction it moves in, simply by modulating their pelvis motion. The control system also provides a means for applying loads to the user's pelvis. For example, defining a virtual pushing force in the control software commands the mobile base to generate a corresponding pushing force at the user's pelvis. This virtual force can be used to propel the user's pelvis during assisted walking, or to perturb the user's gait during balance training.

\subsubsection{The Mobile Base}

The intrinsic motion constraints of gait trainer's mobile base are of concern because they impose motion constraints on the user's body at user-device interface. Though there is ample evidence to suggest that constraining pelvis translation significantly alters gait by inducing longer and narrower steps, increased step time, excessive trunk rotations, and changes in the vertical ground reaction force $[47,51]$, most robotic gait trainers are designed with non-holonomic drive systems that constrain users from moving sideways. Even gait trainers like the WalkTrainer [73] with actuated BWS systems that accommodate natural pelvis motions within a finite volume of space, will impose motion constraints when the 


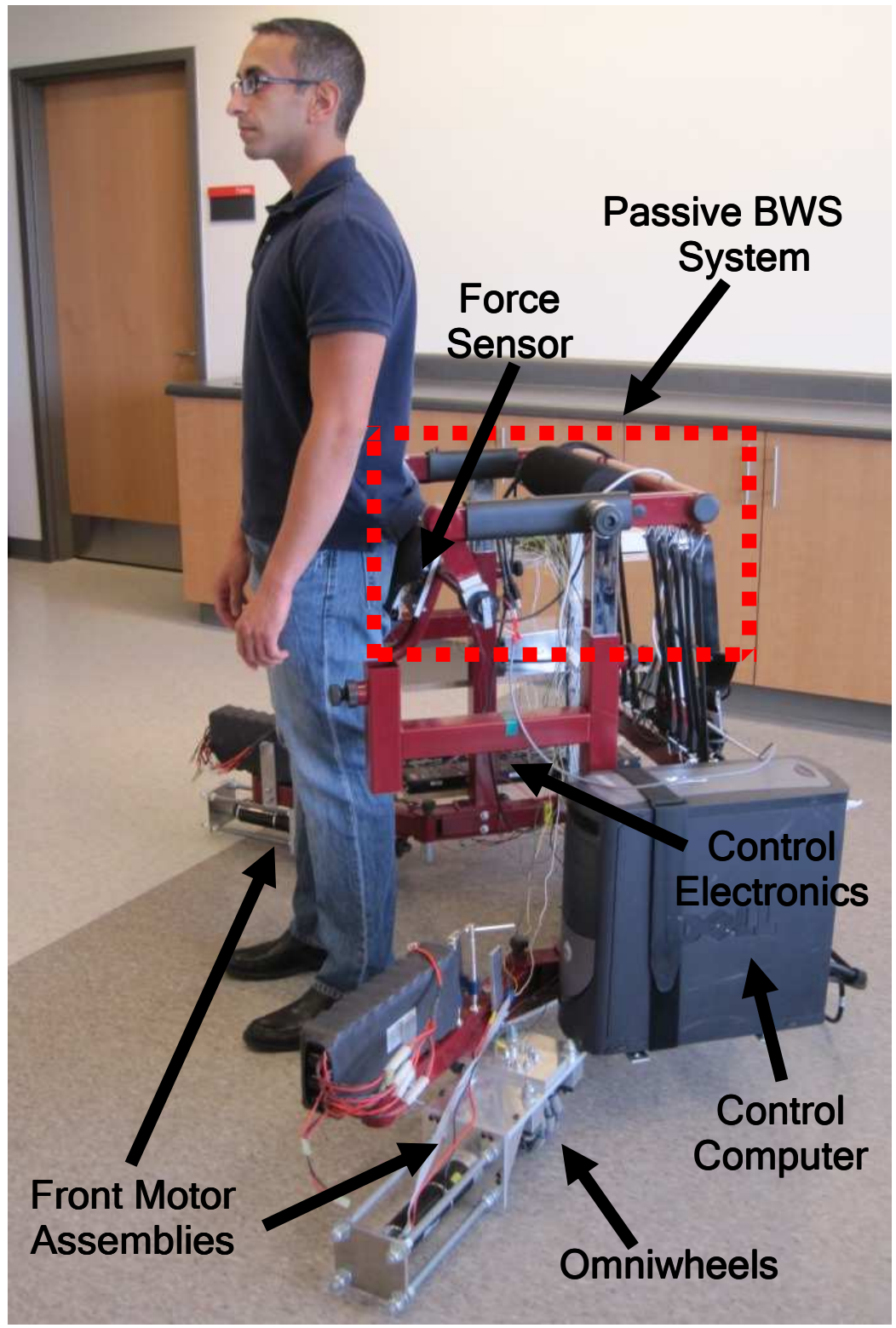

Figure 4.3: An image of the first prototype of the GaitEnable system with its key components highlighted. 
mechanical limits of their BWS systems are exceeded; the imposed motion constraints would be most prevalent during rapid body motions such as change-in-support reactions that require a user to move a large distance quickly. This desire to minimize device-imposed pelvis and torso motion constraints motivated the use of a holonomic mobile base for GaitEnable.

A variety of different mechanisms such as powered castors [168, 169], orthogonal wheels [170], crawler tracks [171], omnidirectional wheels [172], and spherical wheels [173] were investigated when GaitEnable's mobile base was designed. Drive systems with powered castors have a higher load capacity, simpler wheel designs, a better tolerance for ground surface irregularities, and a smoother ride $[168,169]$. However, they require mechanically complex solutions for power delivery and sensing, since the driving wheels must be capable of rotating about an axis perpendicular to the wheel rotation axis $[168,169]$.

Though orthogonal wheels, crawler tracks, and spherical wheels can also be used, wheels that incorporate passive rollers on their periphery (e.g., omniwheels and Mecanum wheels) are most commonly used in mobile robotics applications [174-178]. These drive systems tend to exhibit higher vibrations, reduced traction, and less robustness to variations in the terrain. However, as ground surface irregularities are less prominent in controlled environments such as clinics or hospitals, these drawback were deemed acceptable in this application. Omniwheels were also the most attractive option since they are inexpensive, readily available from several different manufacturers, and simplify the design of the mobile base.

\section{Omniwheel Selection and Configuration}

Rotacaster omniwheels [179] were selected because they provided a larger load capacity at a smaller wheel diameter in comparison to the alternatives, and because they came in pre-assembled units of single, double and triple wheel combinations that could easily be 
interchanged for testing purposes; an image of the Rotacaster single, double, and triple wheels can be seen in Figure 4.4. A variety of wheel combinations were tested prior to selecting Rotacaster's triple omniwheel. Triple wheels with 75A hardness polyurethane rollers were selected as they were observed to generate the least amount of vibrations.
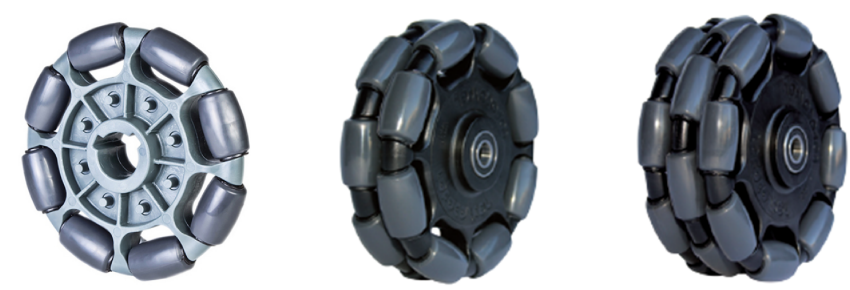

Figure 4.4: An image of the different Rotacaster omniwheels tested on GaitEnable.

Due to a variety of practical limitations such as allowing sufficient leg clearance, avoiding singular wheel configurations, using the available mounting positions, minimizing the risk of tipping, choosing a symmetric configuration, and ensuring an appropriate normal force distribution between the wheels, the wheel positions and orientations were restricted to the configuration depicted in Figure 4.5. This figure presents a top view of the base and identifies the two front wheels rotated at $\pm 225^{\circ}$ with respect to the rear wheel, and the rear wheel aligned with the frontal plane of the user.

\section{Motor Sizing}

A dynamic model of the mobile base was also developed to aid in motor selection. The mobile base was modelled as an unconstrained planar rigid body with a mass of $95 \mathrm{~kg}$ subject to traction forces at the locations of each omniwheel. Normal forces were assumed to be equally distributed at each wheel, and wheel slip models from [180] were used to model omniwheel traction forces with an assumed coefficient of friction of 0.65. A proportional velocity controller was implemented in the simulation, and sinusoidal velocity profiles simulating slow walking speeds under $1 \mathrm{~m} / \mathrm{s}$ were used to estimate the motor torque and 


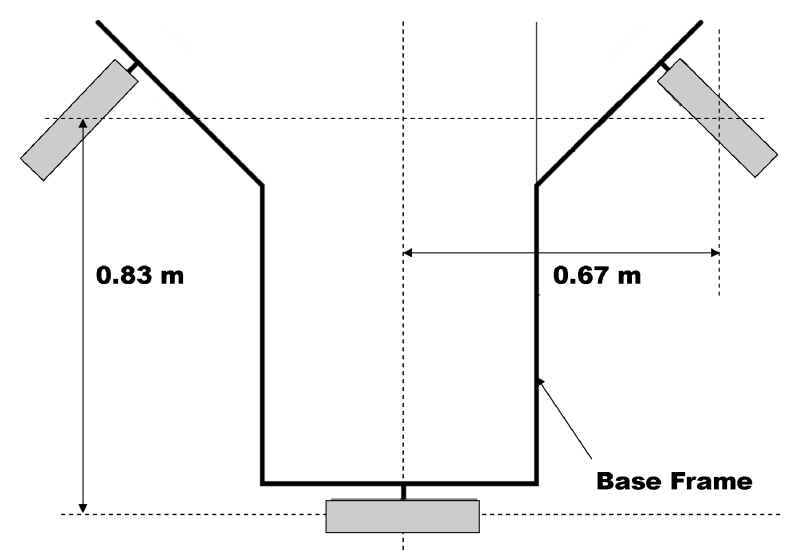

Figure 4.5: An top-view of the mobile highlighting the wheel positions and orientations.

speed requirements. The $1 \mathrm{~m} / \mathrm{s}$ speed limit used to guide the motor sizing was based on average walking speed data for geriatric inpatients [181], a potential patient population for GaitEnable.

The simulation results suggested that peak motor torques of $15 \mathrm{Nm}$ at omniwheel speeds of $13 \mathrm{rad} / \mathrm{s}$ were necessary to accelerate the device to a peak speed of $1 \mathrm{~m} / \mathrm{s}$ in under $1 \mathrm{~s}$. Using these specifications, servomotors comprised of Maxon RE 50 brushed DC electric motors and Maxon GP 52C planetary gearboxes with a 53:1 gear ratio were selected. These motors are capable of generating a continuous torque of $15 \mathrm{Nm}$ at 20 $\mathrm{rad} / \mathrm{s}$. Motors with a maximum speed greater than $13 \mathrm{rad} / \mathrm{s}$ were selected as a precaution, because the required omniwheel speeds can increase significantly when the mobile base simultaneously translates and rotates. As any or all motors could exhibit their peak torque and speed limits during coupled translations and rotations, identical servomotors were used in each omniwheel assembly. For the mobile base configuration described in Figure 4.5, the motor torque specifications allowed a peak forward walking speed of approximately 1.65 $\mathrm{m} / \mathrm{s}$. Using the principle of virtual work and the Jacobian of the mobile base (see Section 4.3.3 for details about the Jacobian), the peak static forces that the mobile base can generate were calculated to be $360 \mathrm{~N}$ in the fore/aft direction and $613 \mathrm{~N}$ in the lateral direction. 
Each servomotor assembly is built as a modular unit that can easily be moved and rotated. The mounts for the motor and wheels are designed as a bolted assembly of machined aluminium parts. A double bearing support is used on each wheel, and a simple cage for protecting the motor is incorporated into the mount design. Figure 4.6 shows an image of an assembled servomotor assembly. While each assembly is designed to support a dynamic load of $125 \mathrm{~kg}$, the maximum patient weight is limited to only $100 \mathrm{~kg}$ to ensure sufficient safety factors on the remaining components of the structure.

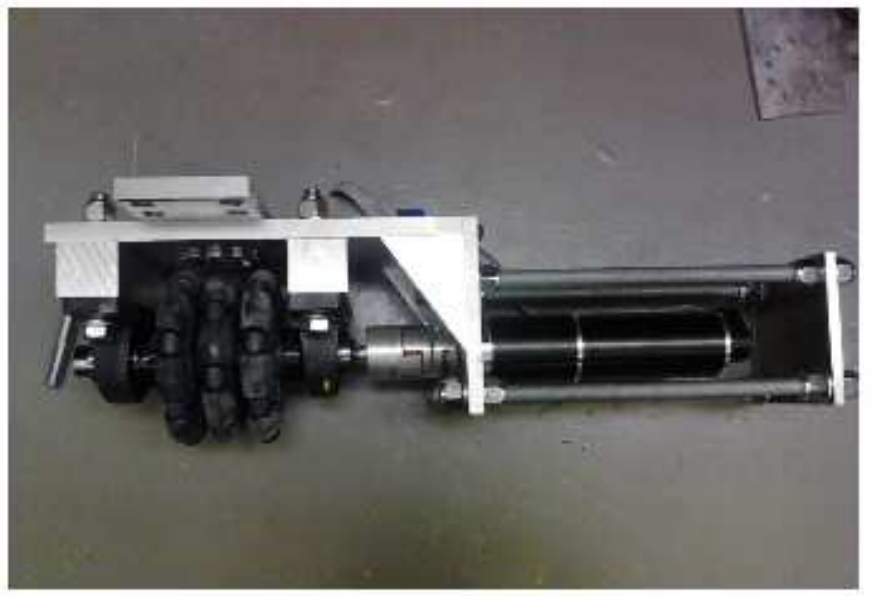

Figure 4.6: An image of an assembled motor assembly.

\section{Robotic System Overview}

The electronics required to control the motors, and implement GaitEnable's interaction control system are summarized in the block diagram in Figure 4.7. The list below provides additional details about the components that make up the robotic system:

- Control Computer: The control computer consists of a PC with a $1.3 \mathrm{GHz}$ Intel Pentium 4 processor that is compatible with a real-time operating system provided by MathWorks Matlab and Simulink software. The National Instruments PCI 6259 and PCI 6601 data acquisition cards installed in the computer act as the 


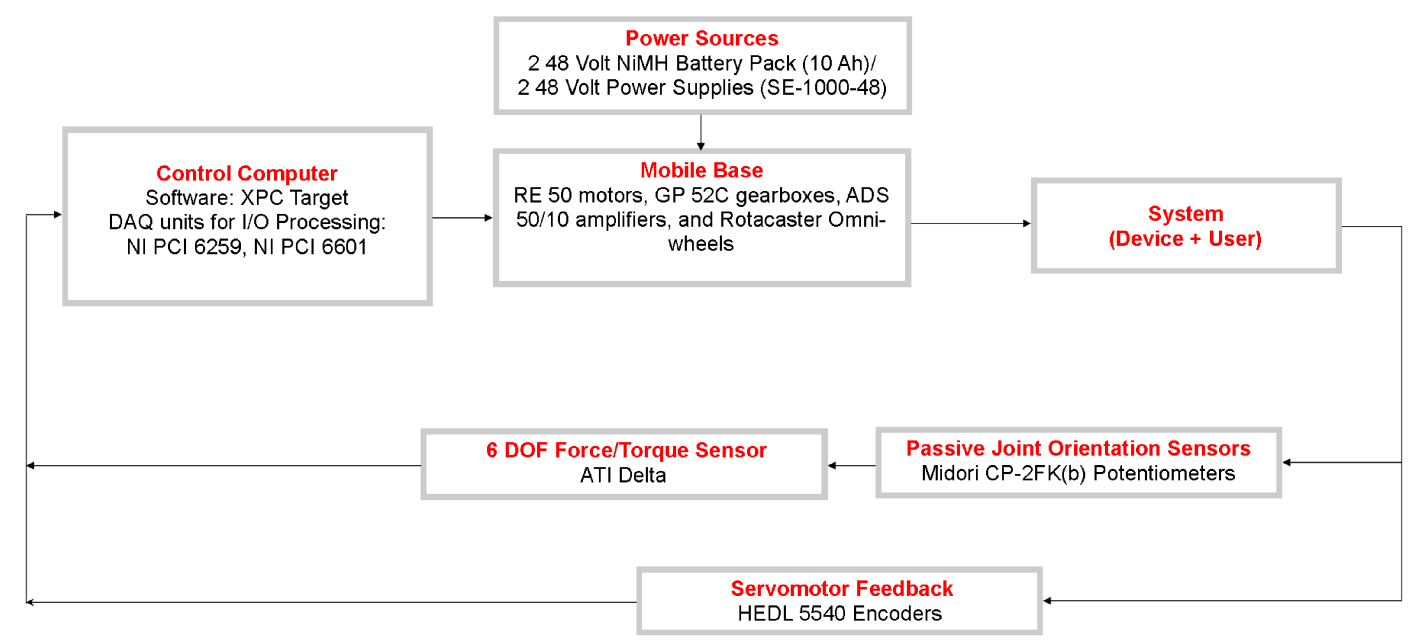

Figure 4.7: A high level overview of the robotic system implemented on GaitEnable.

interface between the sensors and actuators. These data acquisition cards provide 16-bit analog-to-digital conversion circuits with a $\pm 10 \mathrm{~V}$ range for all analog sensor signals, 16-bit digital-to-analog conversion circuits with a $\pm 10 \mathrm{~V}$ range for generating the command signals for the motor amplifiers, and 32-bit counters for counting incremental encoder pulses.

The real-time control software for reading the sensor signals, performing the control calculations, and generating the motor amplifier signals is developed in MathWorks Simulink software using Simulink's xPC Target toolbox and Matlab's Embedded Coder add-on. The xPC Target toolbox provides the drivers for communicating with the NI data acquisition cards, and the Embedded Coder add-on automatically generates $\mathrm{C}$ code that is compatible with the real-time operating system running on the control computer.

The sampling rate of the real-time control system is set to $2000 \mathrm{~Hz}$. Simulink's xPC Explorer graphical user interface is used to enable and disable the code remotely. The entire process of developing, managing, and running the control code follows the 
standard procedure outlined in the xPC Target user manual [182].

- Power Sources: The servomotors can be powered via two 48 V, 10 AH rechargeable Nickel metal hydride batteries, or from two 48 volt/20 A power supplies powered from a standard $120 \mathrm{~V} / 115 \mathrm{~Hz}$ AC wall outlet. The control computer is currently powered via an extension cord attached to a wall outlet. Fully cordless operation would be possible with a power inverter that transforms the DC battery voltage into an $\mathrm{AC}$ voltage suitable for powering the control computer.

- Servomotor Feedback: Incremental optical encoders mounted on each servomotor are used to measure the orientation of the omniwheels. Each encoder has a resolution of 2000 pulses per revolutions. Given the large 53:1 gear ratio, the encoder can measure each omniwheel's orientation with a resolution of $0.000059 \mathrm{rad}$.

Omniwheel angular velocity and is estimated by numerically differentiating encodermeasured orientation. A digital first-order low pass filter with a bandwidth of 30 $\mathrm{Hz}$ is used to de-noise the differentiated signal. The differentiation and filtering is performed within the real-time control.

- Mobile Base: Each Maxon servomotor is controlled by a Maxon ADS 50/10 pulse-width modulated, analog servoamplifier. Each amplifier is operated in torque mode with a maximum current limit of 11 amps corresponding to the maximum continuous current limit of the servomotors. The current command signal for each drive is calculated in the control software and generated at the amplifier input via the digital-to-analog circuitry of the NI PCI 6529 data acquisition card.

- 6 DOF Force/Torque Sensor: GaitEnable's admittance controller requires 
measurements of the forces and torque that the user applies to the device. As discussed in further detail in the following section, a ATI Delta 6 DOF force/torque sensor is mounted behind the structure that constrains the user's pelvis to the device. Thus, the force sensor measures the forces and torques that the user applies to the pelvis constraint structure, and the inertial loads of the constraint structure. As the user's pelvis is offset from the sensor, the loads are assumed to be applied at a fixed offset with respect to the sensor during any data processing. No compensation for the inertial loads is implemented as the system does not have any sensors for measuring the acceleration of the constraint structure.

The ATI Delta 6 DOF force/torque sensor was selected because of its high overload capacity and because it has built-in amplifiers that provide a voltage output signal between $\pm 5 \mathrm{~V}$. Thus, an additional amplification stage for the small voltage signals from strain gauges inside the sensor structure is not required. The six outputs of the sensor are connected to the analog inputs of the PCI 6259 card. A calibration matrix is applied in the control code to calculate the sensed forces and torques.

- Passive Joint Orientation Sensors: If the force/torque sensor is attached to the passive linkage as shown in Figure 4.3, then the sensor will translate and rotate about the sagittal plane when the passive linkage moves with respect to the device. Thus, orientation measurements of the passive linkage's joints are necessary for calculating the transformation matrix that relates the force and torque measurements from the rotated, sensor-fixed axes to a set of axes fixed to the mobile base. Accordingly, Midori CP-2FK(b) rotary potentiometers was installed at each joint of the passive linkage for measuring the joint's orientation. Calibration experiments for relating potentiometer voltages to joint angles were performed independently for each joint using a Mastercraft Torpedo digital level with an accuracy of $\pm 0.1^{\circ}$. 


\subsubsection{The User-Device Interface}

The user-device interface denotes the area of contact between the user and the device. As GaitEnable is an admittance-controlled device, the force the user applies at that contact interface is measured and used to generate GaitEnable's motion command. Thus, the design of this interface also has significant effect on the performance of GaitEnable's interaction control system. The type of factors considered during the design of the interface included the following:

1. Ability to transmit loads to and from the device and ensure a secure connection between the patient and the device.

2. Adequate padding to ensure that the interface is comfortable for the duration of the training period.

3. Avoiding unnecessary motion constraints.

4. Ability to interface to the BMT's BWS system.

The interface shown in Figure 4.8 is the interface used during early experiments [183]. It provided a one-sided connection to the passive linkage. As can be seen in the figure, this interface consisted of padded support, and a 6-DOF force/torque sensor mounted between the padded support and the frame of the BWS system's passive linkage. Since the belts used to secure the user to the padded support had to be kept loose to avoid excessive constraints on the user's hip motions, this interface provided poor lateral support and caused unwanted mobile base rotations when users attempted to side-step.

In order to ensure a better load transfer and to reduce the unwanted base rotations, a new interface with a rigid pelvis support was developed. This interface provided a multi-lateral connection to the user and is shown in Figure 4.9. It consists of a cloth belt surrounded by a u-shaped rigid support made of steel tubing. The 6 DOF force/torque sensor was attached in between the u-shaped support and the frame of the BWS system's 

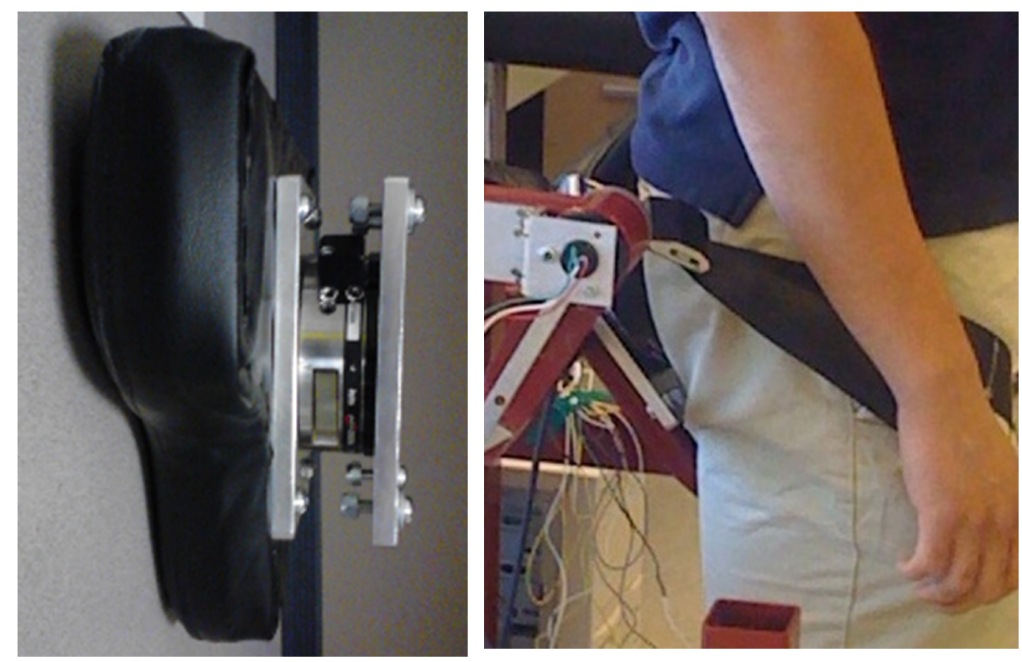

Figure 4.8: A one-sided interface connected to the passive linkage. Users rested their lower back against the black padded support and used two belts that wrapped around their thighs to fix themselves to the padded support. A 6 DOF force/torque sensor mounted between the padded support, and the frame of the BWS system was used to measure the interaction forces and torques.

passive linkage. The two arms of the rigid interface could be rotated to conform to each user's pelvis, and gently constrained users from moving sideways relative to the device. The soft harness surrounding the metal structure provided padding and a means for securing the user to the device. As the padded belt sat at or above the user's waist, this interface also imposed fewer constraints on hip flexion and extension. Users also noted that side-stepping was easier, and that the connection to the device felt more secure.

A third interface which featured a multi-lateral connection to the user, and a direct connection to the robot frame is shown in Figure 4.10. This interface also consisted of a waist belt surrounded by a u-shaped rigid support. However, it bypassed the passive linkage, and was installed on the fixed arm supports of the mobile base. Thus, it generated the most direct connection between the user and the device, and was used to remove the influence of the passive linkage. It was used in most experiments because it provided a more direct measure of the mobile base's ability to follow and assist the user's voluntary motion. 

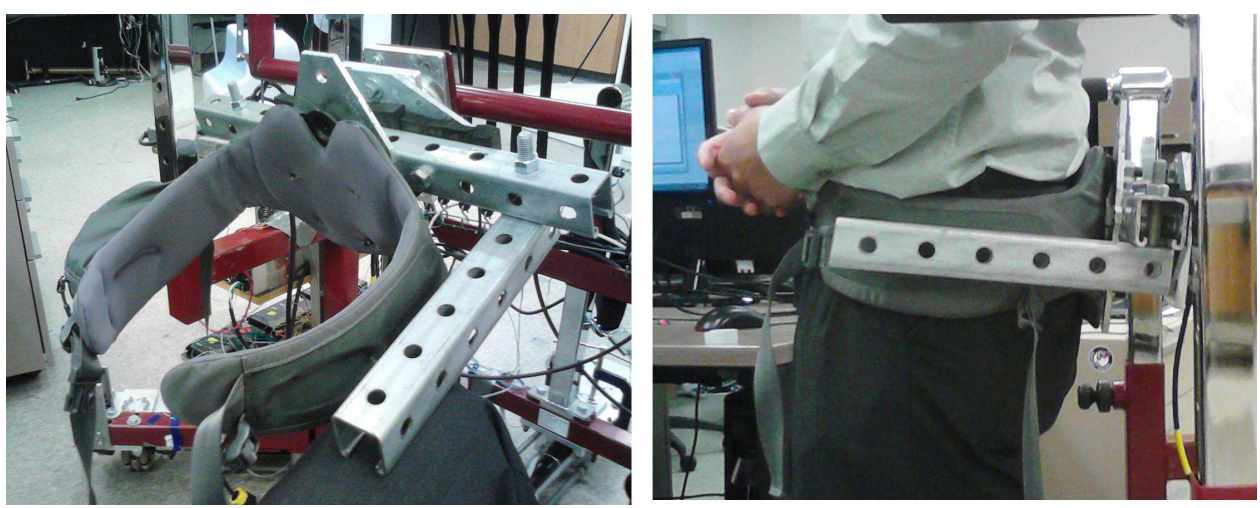

Figure 4.9: A multi-lateral interface connected to the passive linkage. Users strapped the belt around their waste and pushed the side arms of the u-shaped support around their waist to ensure a more rigid connection to the device.

As shown in Chapter 5, a comparison of the multi-lateral interface connected to the passive linkage with this interface provides a means for isolating the effects of the passive linkage.

\subsection{GaitEnable's Interaction Control System}

As noted in Chapter 2, interaction control systems for rehabilitation robots such as GaitEnable should provide a stable interaction, a simple and intuitive mechanism for the user to control the motion of the robot, the ability assist rather command the user's motions, and provide a means for relating therapy-specific requirements into corresponding robot motion commands. The enhanced admittance control presented in Chapter 3 provides an appropriate solution that satisfies all these requirements.

The application of this admittance controller on GaitEnable is summarized in the block diagram shown in Figure 4.11. The force/torque sensor mounted behind the user's pelvis measures the interaction forces and torque generated at the contact interface. These measurements and the feedforward inputs $F_{v}$ are used in the interaction model to generate the reference acceleration $\ddot{x}_{r}, \ddot{y}_{r}$, and $\ddot{\theta}_{r}$ in a Cartesian frame. The reference acceleration is transformed into the corresponding desired omniwheel accelerations via the Jacobian, the kinematic relationship between the omniwheel velocities and the mobile base velocity. 


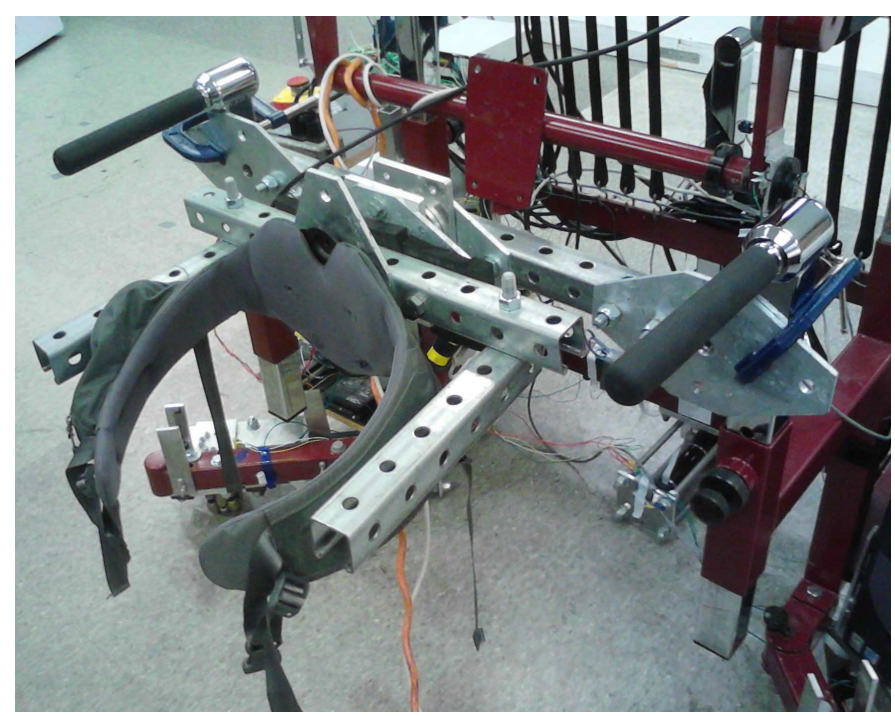

Figure 4.10: A multi-lateral interface connected to the robot frame. This interface consists of a cloth belt surrounded by a u-shaped rigid support. The interface is attached to the mobile base's fixed arms supports so that the motion of the passive linkage does not influence the interaction.

The desired omniwheel accelerations are then passed through the impedance manipulations described in Chapter 3, and numerically integrated to generate the desired omniwheel angular velocities and orientation. A proportional position controller at each servomotor assembly is implemented to track the desired omniwheel trajectories. The sections below provide additional details about these different operations.

\subsubsection{Equations of Motion}

If GaitEnable is modeled as a planar mobile manipulator with coordinate frames as defined in Figure 4.12, and if the user is assumed to be attached to the passive linkage, then the results from [184], which describe a process for modeling mobile manipulators, may be used directly. After accounting for the effects of the mobile base's motion on the passive linkage, the equations of motion of the passive linkage take on the following form: 


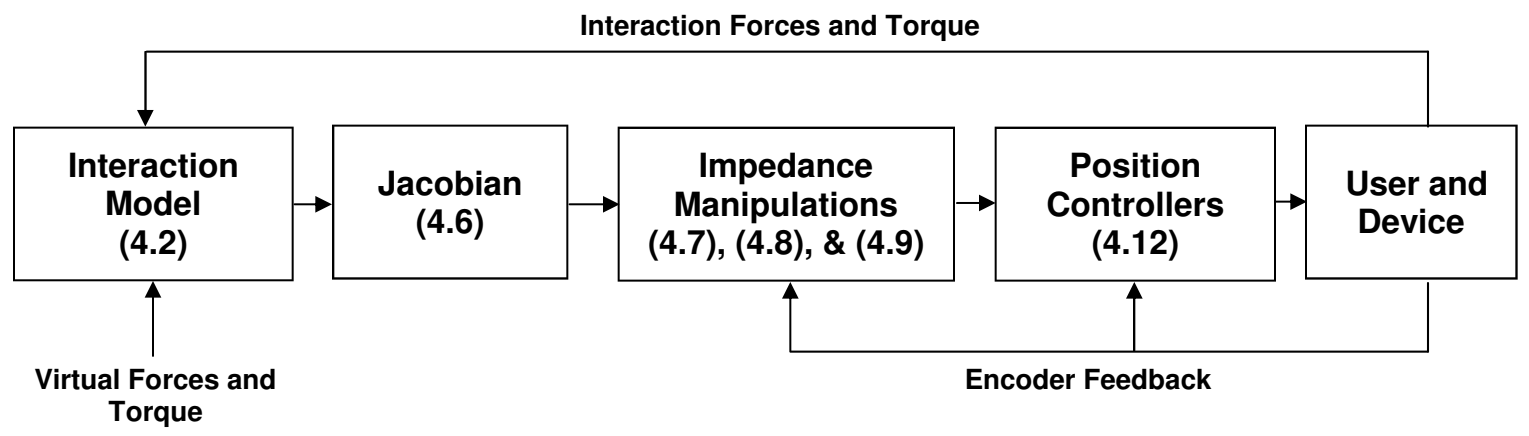

Figure 4.11: A block diagram of the admittance controller.

$$
M\left(q_{m}\right) \ddot{q}_{m}+C_{1}\left(q_{m}, \dot{q}_{m}\right)+C_{2}\left(q_{m}, \dot{q}_{m}, \dot{q}_{b}\right)+R_{r}\left(q_{m}, q_{b}\right) \ddot{q}_{b}+K\left(q_{m}\right)=f_{t}+R_{\mathbf{X}^{\prime} \mathbf{Y}^{\prime}}^{\mathrm{XY}} F_{i}
$$

where $q_{b}=[x, y, \theta]^{T}$ is the position and orientation of GaitEnable's centre-of-mass (CoM) expressed with respect to a global coordinate frame, and $q_{m}$ is a vector of the joint angles of the BWS system's 3-DOF passive linkage. $f_{t}=\left[f_{x}, f_{y}, \tau_{z}\right]^{T}$ denotes the traction forces and torque generated by the 3 actuated omniwheels and $F_{i}=\left[f_{x, i}, f_{y, i}, \tau_{\theta}\right]^{T}$ are the interaction forces and torque generated at the user-device interface, and $R_{\mathrm{X}^{\prime} \mathrm{Y}^{\prime}}^{\mathrm{XY}}$ is a rotation matrix which transforms quantities from the device-fixed frame to the global frame. $M\left(q_{m}\right)$ denotes the passive linkage's inertia matrix, $C_{1}\left(q_{m}, \dot{q}_{m}\right)$ denotes the Coriolis and centrifugal terms of the passive linkage, and $K\left(q_{m}\right)$ denotes the gravity terms and stiffness terms arising from the bungee cords. $R_{r}\left(q_{m}, q_{b}\right)$ and $C_{2}\left(q_{m}, \dot{q}_{m}, \dot{q}_{b}\right)$ are the terms that represent the dynamic interaction caused by the motion of the mobile base. They can be derived using the procedure outlined in [184].

As the enhanced admittance control presented in Chapter 3 does not require a model of the device's dynamics, a model of GaitEnable was not derived for control synthesis. The general form of the model is presented here solely to demonstrate that the passive linkage's dynamics, which the interaction control attempts to shape, are multi-DOF, nonlinear, 


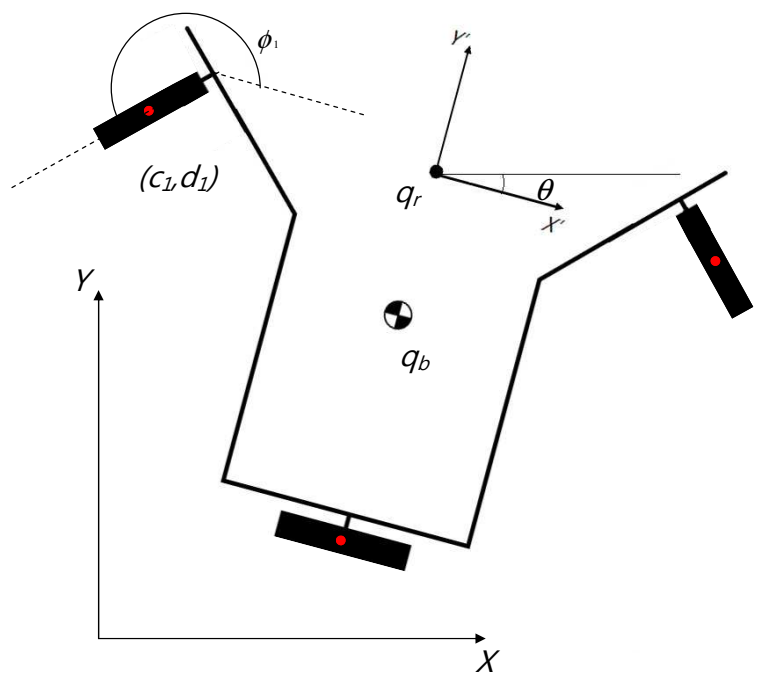

Figure 4.12: The top view of the mobile base. This sketch illustrates the relative positions of the user's pelvis (i.e., $q_{r}$ ), the device's CoM, and the omniwheel assemblies.

and coupled, even before considering the added complications of omniwheel slip and the additional dynamics that arise from the coupling of the position controllers, interaction model, and user dynamics. In other words, GaitEnable is a very challenging platform for testing the impedance manipulations.

\subsubsection{The Interaction Model}

The interaction forces and torques developed at the user-device interface are measured by an ATI Delta 6-axis force/torque sensor mounted between the pelvis constraint structure and the frame of the device. The three components of the measured interaction forces and torques associated with the device's motion in the ground plane, $F_{i}=\left[f_{x, i}, f_{y, i}, \tau_{\theta}\right]^{T}$, are used as inputs to the following interaction model:

$$
M_{r} \ddot{q}_{r}+C_{r} \dot{q}_{r}=F_{i}+F_{v}
$$


where $q_{r}=\left[x_{r}, y_{r}, \theta_{r}\right]^{T}$ is a reference point on the robot where the interaction model is assumed to hold, $M_{r}=\operatorname{diag}(m, m, j)$ denotes a diagonal matrix that corresponds to the inertia matrix of the interaction model, and $C_{r}=\operatorname{diag}\left(c, c, c_{\theta}\right)$ denotes a diagonal matrix that corresponds to the damping matrix of the interaction model. $m, c, c_{\theta}$ and $j$ are selectable parameters that determine the device impedance sensed by the user, and $F_{v}=\left[f_{x, v}, f_{y, v}, \tau_{z, v}\right]^{T}$ are the designer-specified feedforward inputs that provide force cues to assist, perturb or stabilize a user's gait. The matrices $M_{r}$ and $C_{r}$ denotes the desired mass/inertia and damping that GaitEnable should display to the user.

Equation (4.2) specifies the desired dynamics of the device and is numerically integrated in time to generate the mobile base's motion commands $\dot{x}_{r}, \dot{y}_{r}$, and $\dot{\theta}_{r}$. These reference velocities represent the desired base motion that should be observed for a given interaction force/torque trajectory. If the reference point $q_{r}$ is assumed to be coincident with GaitEnable's centre of mass (CoM) $q_{b}$, and if GaitEnable's velocity controller is assumed to allow perfect tracking of $\dot{x}_{r}, \dot{y}_{r}$, and $\dot{\theta}_{r}$, then if follows that $q_{b}=q_{r}$ and that the mobile base displays the impedance specified in the interaction model to the user. In other words, the mobile base is driven by the feedforward inputs $F_{v}$, and displays the inertial and damping properties specified in $M_{r}$ and $C_{r}$.

The reference point is selected to be the approximate midpoint of the support polygon formed by the subject's feet. This point corresponds to the projection of the user's pelvis CoM onto the ground plane during double stance. Defining the reference point in this way allows the moment input $\tau_{\theta}$ to generate rotation commands that are centered about the user's pelvis.

Since (4.2) reshapes the interaction dynamics to include the effects of the feedforward inputs $F_{v}$, the user will also feel $F_{v}$ being applied to their pelvis. This is the means by which the admittance controller can display additional therapy-dependent force cues. For example, a small, slowly varying force along the $x_{r}$ axis (e.g., setting $f_{x, v}=5 \sin 0.1 \pi \mathrm{t}$ ) may 
be used for training lateral weight shift, or a constant pushing force along the $y_{r}$ axis may be used to assist the user's forward walking motion as in [185].

\subsubsection{Decomposing Reference Velocities to Corresponding Omniwheel Velocities}

Once the reference acceleration $\ddot{x}_{r}, \ddot{y}_{r}$, and $\ddot{\theta}_{r}$ have been computed, they must be decomposed into corresponding desired omniwheel accelerations. The kinematic relationship that relates the reference accelerations to the desired omniwheel velocities - the Jacobian in Figure 4.11 - is unique to the wheel configuration and selected reference point, and may be derived for each omniwheel assembly using the procedure described in [186]. This derivation yields an expression of the form:

$$
\dot{q}_{r}=r_{w} J \dot{q}_{w}
$$

where $J$ denotes the Jacobian matrix, $\dot{q}_{w}$ is a vector of omniwheel angular velocities, and $r_{w}$ is the radius of the omniwheels. GaitEnable's control system requires transforming the desired base velocity into corresponding omniwheel trajectories. This can be achieved via the inverse of the Jacobian as follows:

$$
\dot{q}_{w}=\frac{J^{-} 1}{r_{w}} \dot{q}_{r}
$$

with the general expression for $J^{-1}$ given as 


$$
J^{-1}=\left[\begin{array}{ccc}
-\sin \left(\phi_{1}\right) & -\sin \left(\phi_{2}\right) & -\sin \left(\phi_{3}\right) \\
\cos \left(\phi_{1}\right) & \cos \left(\phi_{2}\right) & \cos \left(\phi_{3}\right) \\
c_{1} \cos \left(\phi_{1}\right)+c_{1} \sin \left(\phi_{1}\right) & c_{2} \cos \left(\phi_{2}\right)+d_{2} \sin \left(\phi_{2}\right) & c_{3} \cos \left(\phi_{3}\right)+d_{3} \sin \left(\phi_{3}\right)
\end{array}\right],
$$

where $\phi_{i}, c_{i}$, and $d_{i}$ in Equation (A.1) denote the orientation and position of the $i^{\text {th }}$ omniwheel with respect to the reference point $x_{r} . \phi_{1}, c_{1}$, and $d_{1}$ are shown in Figure 4.12. The red dots in this figure denote the wheel-ground contact points. Since the Jacobian is time-invariant, the following equation may be used to relate the reference acceleration to the corresponding desired omniwheel accelerations:

$$
\ddot{q}_{d}=\frac{1}{r_{w}} J^{-1} \ddot{q}_{r}
$$

where $\ddot{q}_{d}$ denotes the desired omniwheel acceleration.

\subsubsection{Manipulating Desired Omniwheel Accelerations to Guarantee Sta- ble Interactions}

Ensuring that GaitEnable displays a small impedance is essential since to allow a patient to easily move and walk with GaitEnable. In Chapter 3, it was noted that typical admittance control implementations are poor at displaying small impedances. However, Chapter 3 also described two reference trajectory manipulations that allow admittance-controlled robotic devices to safely display small impedances. These manipulations were also implemented in GaitEnable's control system to allow it to display a small apparent mass/inertia.

Error feedback in the reference model is first applied to the omniwheel accelerations $\ddot{q}_{d}$ to attenuate oscillations. This method of reshaping the impedance was presented in Section 
3.3 and requires that $\ddot{q}_{d, i}$ is manipulated as

$$
\ddot{q}_{t, i}= \begin{cases}\ddot{q}_{d, i}+b_{w} \dot{s}_{w, i}, & \text { if } s_{w, i} \dot{s}_{w, i}>0 \\ \ddot{q}_{d, i}, & \text { if } s_{w, i} \dot{s}_{w, i} \leq 0\end{cases}
$$

where $\ddot{q}_{t, i}$ is the manipulated desired omniwheel acceleration used to generate the desired trajectory of the omniwheels, $b_{w}$ is the gain of the stabilizing feedback, and the subscript $i$ denotes the $i^{t h}$ omniwheel. The quantity $s_{w, i}$, the equivalent of the filtered tracking error $s_{q, i}$ in Chapter 3, is defined as follows:

$$
\begin{aligned}
s_{w, i} & =\left(\dot{q}_{t, i}-\dot{q}_{w, i}\right)+\phi_{w}\left(q_{t, i}-q_{w, i}\right) \\
& =e_{w, i}+\phi_{w} \dot{e}_{w, i}
\end{aligned}
$$

where $\phi_{w}$ is a positive constant that controls the bound on the omniwheel tracking error $e_{w, i}$. The derivative of the filtered tracking error, $\dot{s}_{w, i}$, was low-pass filtered before it was used in 4.7.

Next, the manipulated reference omniwheel accelerations $\ddot{q}_{t}$ were constrained using the acceleration limits described in Section 3.2.2. These limits were applied independently for each omniwheel as follows:

$$
\ddot{q}_{t, i}>-\phi_{w} \dot{e}_{w, i}-\psi_{w} \operatorname{sgn} s_{w, i}-\gamma_{w} s_{w, i}-\eta_{w},
$$

$$
\ddot{q}_{t, i}<-\phi_{w} \dot{e}_{w, i}-\psi_{w} \operatorname{sgn} s_{w, i}-\gamma_{w} s_{w, i}+\eta_{w},
$$


where and $\gamma_{w}, \psi_{w}$, and $\eta_{w}$ are analogous to $\gamma, \psi$, and $\eta$ in Section 3.2.2. Application of the acceleration limits allows Theorem 1 from Chapter 3 to be invoked. Thus, it follows that if (4.9) and(4.10) are used to limit $\ddot{q}_{t, i}$, then the filtered tracking error $e_{w, i}$ must be bounded as follows:

$$
\left|e_{w, i}\right| \leq \frac{\eta_{w}}{\gamma_{w} \phi_{w}}
$$

This result confirms that the stability of the $i^{\text {th }}$ omniwheel's position control loop.

The control system described in Figure 4.11 is effectively open-loop since an additional velocity feedback is not used to correct mobile base velocity tracking errors (i.e., there is no separate control loop for ensuring accurate tracking of $\dot{x}_{r}, \dot{y}_{r}$, and $\left.\dot{\theta}_{r}\right)$. Thus, the mobile base will only exhibit the desired impedance specified in Equation (4.2) if the omniwheels do not slip excessively and if the impedance manipulations do not significantly limit the device's motion. Numerous different localization and slip estimation algorithms [187-189] could be used for estimating the mobile base's velocity and for implementing a closed-loop velocity controller. However, the added sensing and computational complexities that these solutions impose were avoided as it is far simpler for the user to apply corrective force inputs to help compensate for the velocity tracking errors.

\subsubsection{Omniwheel Position Controllers}

The proportional controllers used at each omniwheel were implemented as follows:

$$
V_{m, i}=K_{p, i} e_{w, i}
$$


where $V_{m, i}$ is the voltage command sent to the $i^{\text {th }}$ motor's amplifier. The motor amplifier interprets this voltage signal as a current command for the motor.

\subsection{Preliminary Experiments}

The experimental results in Chapter 3 demonstrated that the user-device interaction in an admittance-controlled haptic device can exhibit a violent instability when a user forcefully pushes or pulls against a haptic device. It was also shown in Chapter 3 that haptic devices approach their stability limits when required to display a small apparent inertia. GaitEnable must display a small inertia so that it is easy for patients to move, and is subject to sudden and large force input in many situations such as when a user performs a change-in-support reaction. As such, GaitEnable continuously operates in a condition in which its control system is pushed to its stability boundaries. The experiment described below demonstrates that stable interactions are possible even if the user intentionally tries to destabilize the interaction.

\subsubsection{Experiment Overview}

The test conditions considered were particularly challenging because the desired mass of GaitEnable was tuned to the smallest value that could reasonably be rendered within the motor's current limits, and because the user was requested to intentionally perform an activity that is known to destabilize the interaction. During the experiment, a healthy male subject was instructed to push GaitEnable away from his body as hard as possible and subsequently hold GaitEnable in position. For safety reasons, the subject was not attached to the device, and was requested to push on the device while holding onto the pelvis harness with his hands. A research assistant continuously monitored the experiment and was responsible for depressing an emergency switch to disable the servomotors in case 
of an adverse event. While this experiment only considers results from one participant and gain setting, additional experiments described in Chapter 5 confirm that subject-specific effects, and changes in controller parameters do not destabilize the user-device interaction.

The subject was requested to repeat this task at two test conditions. In the first condition - denoted as Without EF in all figures - the controller parameter $b_{w}$ was set to zero to remove any error feedback in the reference model. In this case, only the acceleration limits (4.9) and (4.10) were used to stabilize the interaction. In the second test condition - denoted as With EF in all figures - the parameter $b_{w}$ was set to 67 to help attenuate oscillations. As such, the experiments compared the stability of the interaction with and without error feedback in the reference model. Experiments with a conventional $\mathrm{P}$ controller were not performed as GaitEnable exhibited a violent instability as soon the subject attempted to grasp the harness.

The remaining controller parameter were specified as $K_{p, i}=52.4 \mathrm{~V} / \mathrm{deg}, m=4 \mathrm{~kg}$, $c=0 \mathrm{Nm} / \mathrm{s}, j=2 \mathrm{kgm}^{2}$, and $c_{\theta}=5 \mathrm{Nmrad} / \mathrm{s}^{2}$. The $\mathrm{P}$ gain $K_{p, i}$ was incrementally increased to the largest value that did not generate any noticeable chatter. The parameters of the acceleration limits were specified as $\psi_{w}=90 \frac{\mathrm{rad}}{\mathrm{s}^{2}}, \phi_{w}=40, \gamma_{w}=250 \frac{1}{\mathrm{~s}}$, and $\eta_{w}=500 \frac{\mathrm{rad}}{\mathrm{s}^{2}}$. Identical acceleration limit and $\mathrm{P}$ gains were used for all three omniwheel position controllers as identical servomotors were used to power all three omniwheel assemblies. For the given controller parameters, Theorem 1 and (3.18) imply that the peak tracking error at each omniwheel should have been less than 2.86 degrees. Results from the experiments are shown in Figure 4.13.

\subsubsection{Results and Discussion}

Tracking error data from all three omniwheels are presented in Figure 4.13a, and show that the tracking errors are highly oscillatory at the without EF test condition when $b_{w}=0$ and no error feedback is used to reshape the reference acceleration. The oscillations were initiated at the instant that the user first held onto the harness 5 seconds 


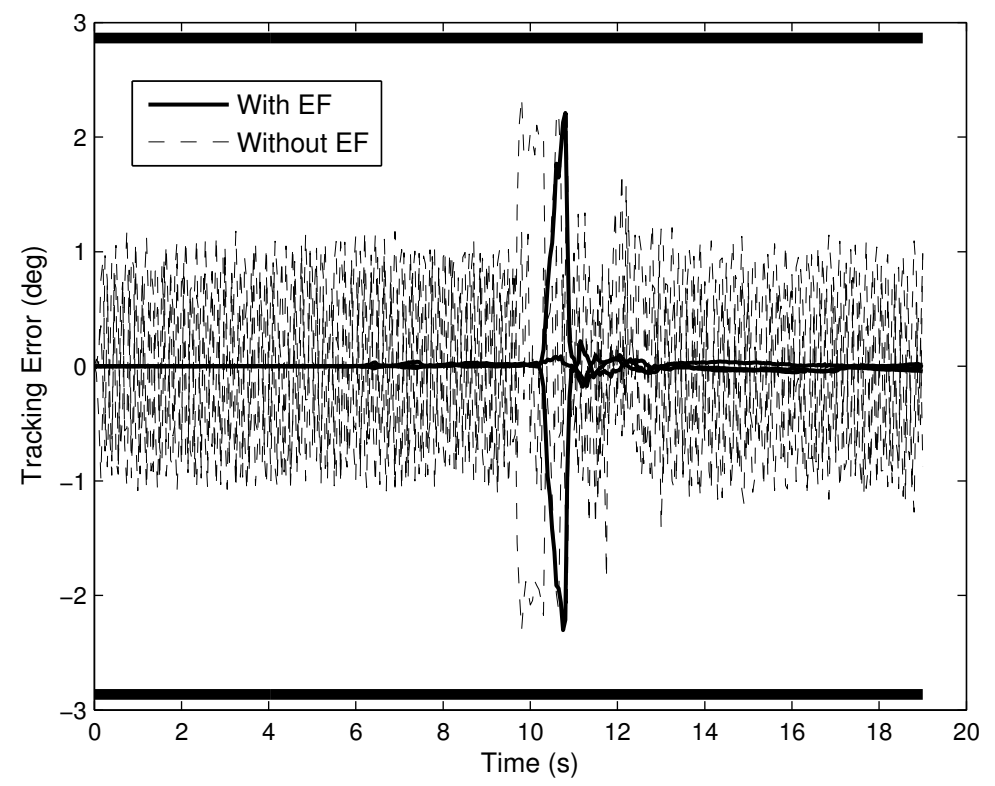

(a) Tracking Error

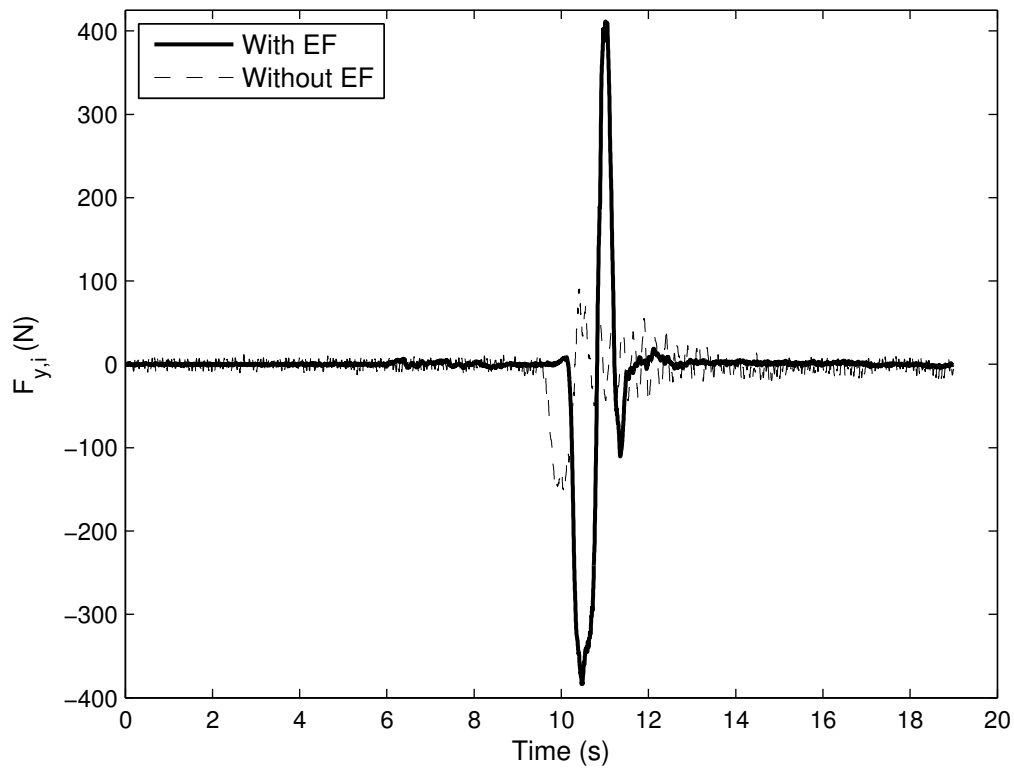

(b) Interaction forces in Sagittal Plane

Figure 4.13: Comparing controller performance with and without error feedback (EF) in the reference model. Detailed caption can be found on the next page. 


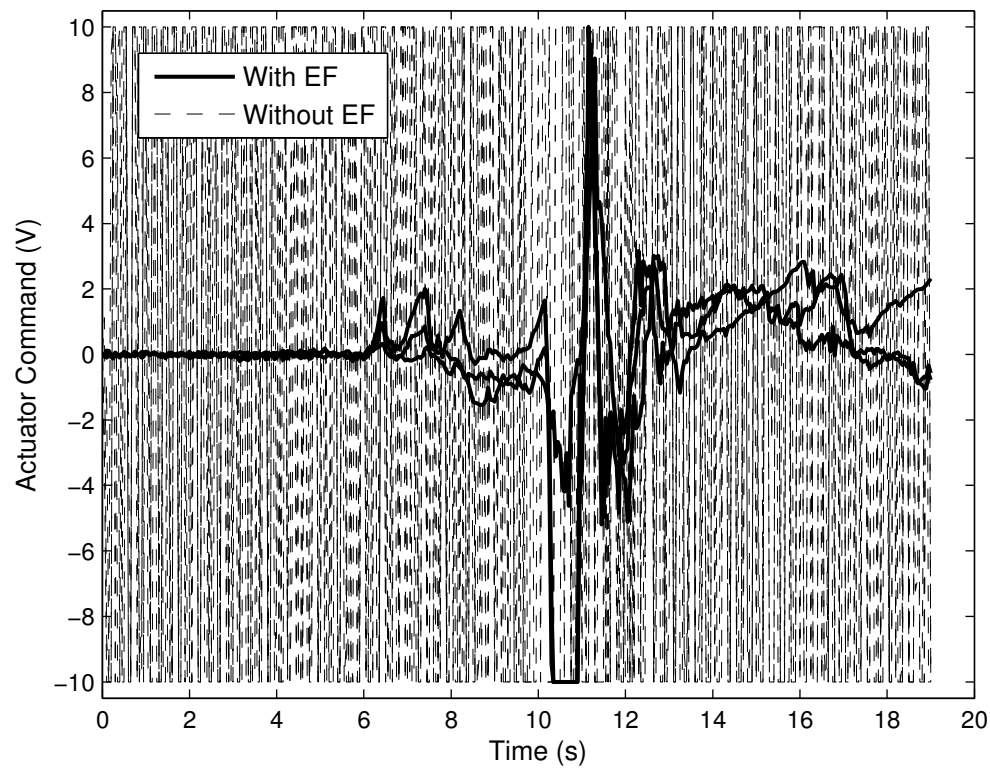

(c) Actuator Commands

Figure 4.13: Comparing controller performance with and without error feedback (EF) in the reference model. (a) All omniwheel tracking errors. The thick dark lines denote the theoretical error bounds. (b) Interaction force in the sagittal plane. (c) Actuator commands for all omniwheel servomotors. While the omniwheel tracking errors remain bounded within the theoretical error bounds, the interaction is highly oscillatory and unsafe without error feedback. Error feedback attenuates the sustained oscillations, and results in a stable interaction, even if the actuators are saturated. 
into the test, and grew once the user pushed GaitEnable 8 seconds into the test. The oscillations continued for the remainder of the test and showed no sign of subsiding. The interaction force data and actuator command data in Figures $4.13 \mathrm{~b}$ and $4.13 \mathrm{c}$ demonstrate the same pattern, and confirm that the interaction is highly oscillatory. The $30 \mathrm{~N}$ amplitude oscillations observed in the interaction force data after the participant's push are of concern because they could be sufficient to perturb an impaired individual's gait, or otherwise cause injuries. Thus, while the tracking errors always remain within their theoretical bounds of $\pm 2.86^{\circ}$, the interaction is unsafe when there is no error feedback.

When error feedback is applied to the reference acceleration, there are no oscillations, and the user can safely push and hold the device in place. This can be observed in Fig $4.13 \mathrm{~b}$ from the way the interaction force settles to a constant value after 15 seconds. Moreover, the stability of the interaction is not compromised even though the actuators are saturated around 13.5 seconds into Figure 4.13c. A comparison of the results between the two test conditions confirms that the acceleration limits and error feedback combined are essential for ensuring a stable interaction. Though lower $\mathrm{P}$ gains at the without EF test condition may yield less oscillatory interactions, they would make GaitEnable less responsive when the user applies smaller force inputs to move GaitEnable. Thus, using higher $\mathrm{P}$ gains with the acceleration limits provides a better trade-off.

Figure $4.13 \mathrm{~b}$ shows the peak forces during the push are $-150 \mathrm{~N}$ at the without EF test condition, and $-385 \mathrm{~N}$ at the with $\mathrm{EF}$ test condition. The higher forces observed at the with EF test condition imply that the device felt heavier at this test condition. This is a result of the more stringent reference acceleration limits imposed by the error feedback term. Thus, the trade-off for ensuring a stable interaction is that the device feels heavy during the pushing motion.

The results of this experiment mirror the results in Section 3.4.5, and demonstrate that the acceleration limits guarantee bounded tracking errors, and that error feedback helps 
attenuate sustained oscillations. The experiments in Section 3.4.5 were performed with a simple robotic device while GaitEnable's dynamics are highly non-linear and coupled. Nevertheless, the impedance manipulations guaranteed the stability of the interaction despite the fact that rudimentary proportional controllers are used for the omniwheels.

As noted in Chapter 3, a stable, model-free control is realized if the impedance manipulations are coupled with simple position controllers. The benefits of this feature are more clear for GaitEnable - neither GaitEnable's mass properties, the passive linkage's dynamics, nor omniwheel slip/skid dynamics have to be accounted for in the design of the omniwheel position controller. However, stabilizing the closed-loop dynamics via the impedance manipulations does come with some drawbacks. There are more parameters to tune, and though general guidelines such as those in Remark 1 can be used to select controller gains, some iterative tuning is inevitable. Additionally, the impedance manipulations reduce the fidelity of the impedance display. Thus, more sophisticated position controllers that provide better tracking performance combined with the impedance manipulations, could result in reduced acceleration limit activations and a more accurate impedance display.

\subsection{The Future of GaitEnable}

GaitTronics Inc., a start-up based in Ottawa is commercializing the GaitEnable technology. A patent cooperation treaty application has also been filed to protect the intellectual property associated with the control system and the device design. Figure 4.14 shows an image of the pre-commercial prototype of the GaitEnable gait trainer developed at Carleton University's Advanced Biomechatronics Laboratory. The research and development of this prototype was funded with an NSERC I2I commercialization grant between July 2012 to August 2013, and was geared towards addressing the unresolved issues with the first prototype. 
This new prototype retains the control architecture and mobility system presented in this chapter. In addition, it also features an admittance-controlled vertical stage capable of lifting patients from their bed and tracking their CoM displacement. The vacuum-formed casing covers the components and protects users from the metal frame of the device. A remote control integrated into the control system enables a therapist to manipulate the patient's motion without physically pushing the device or the patient. This prototype also features enhanced fall-prevention algorithms that use force sensor data, the position of the vertical stage, and the speed of the mobile base to detect and prevent patient falls. Initial patient experiments with local hospitals are slated for February 2014.

Patients in acute care often have chest tubes, intravenous lines and other body-attached devices that need to be moved with the patient, and that can interfere with attachment of the harness. Some patients may also have restrictions that make it impossible for them to wear a chest-based harness. Thus, future versions of the device will be designed to accommodate these body-attached devices and will include interchangeable chest, and pelvis harnesses that give patients the option to be supported from their waist or under their shoulders.

\subsection{Chapter Summary}

This chapter introduced the GaitEnable, a robotic gait trainer composed of a passive BWS system, an omniwheel actuated omnidirectional mobile base, and an interaction control system. The omniwheel-based mobility system implemented on the device and its pelvis-driven interaction control system make it unique with respect to other robotic gait trainers in the literature. As demonstrated further in Chapter 5, the actuated omnidirectional mobile reduces device-imposed motion constraints, and facilitates a more natural gait. The interaction control system also provides an intuitive interface for user's to command the motion of the device - user's move in their preferred direction, and the 


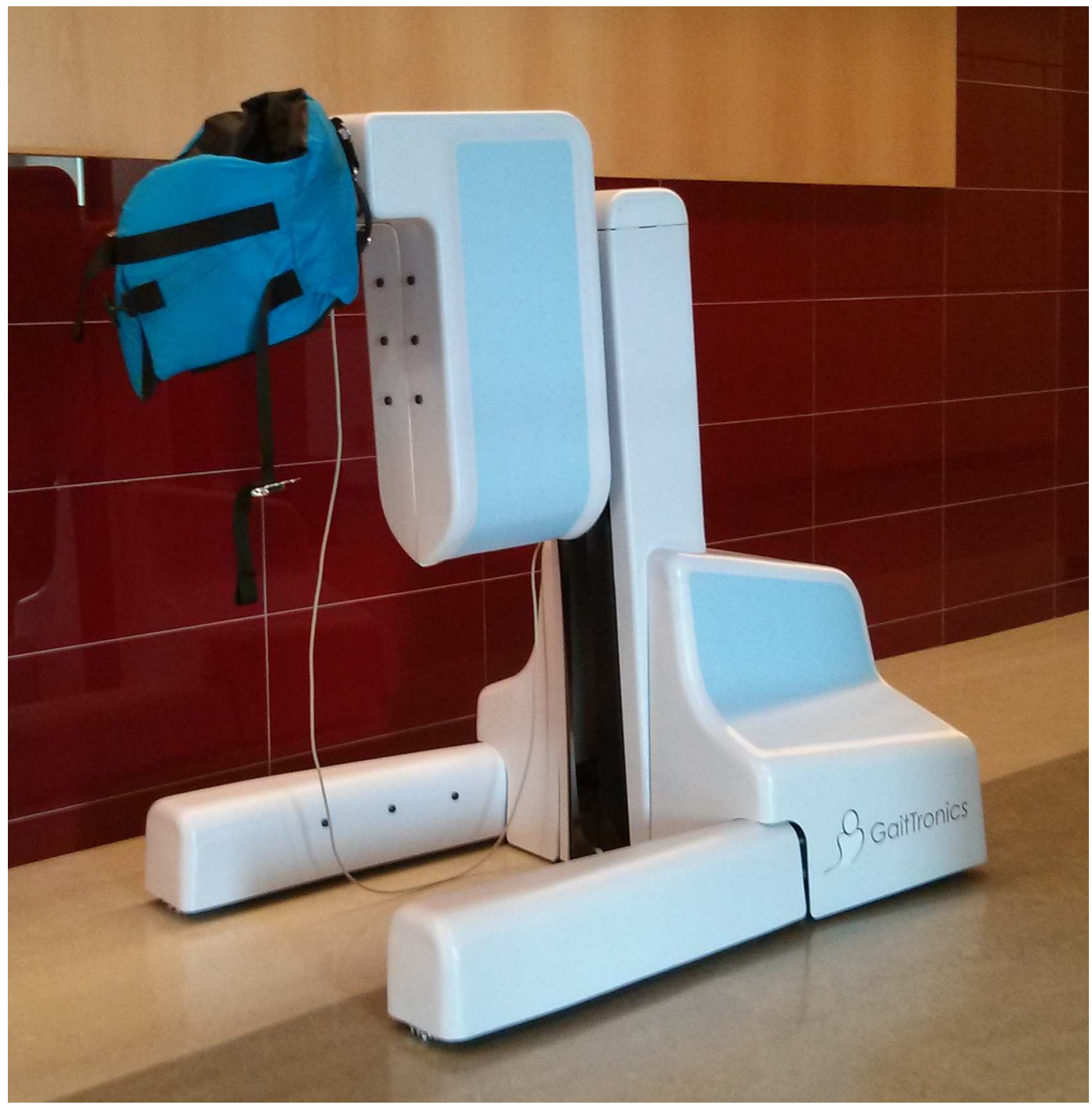

Figure 4.14: An image of the second-generation prototype of the GaitEnable gait trainer. 
device automatically follows. In addition, the control system can generate force cues for assisting or perturbing the user's motion, and experimental results confirm that the impedance manipulations proposed in Chapter 3 allow GaitEnable to safely display a small apparent inertia. In short, the introduction of the actuated omnidirectional mobile base and interaction control system result in a minimal robotic gait trainer that can address several limitations of passive walking aids such as the BMT. 


\section{Chapter 5}

\section{Experiments with GaitEnable}

GaitEnable's mobile base and control system should reduce device-imposed motion constraints, allow users to easily move the device, and generate force cues for influencing the user's gait in a predictable manner. This chapter describes results from a series of experiments performed by healthy participants, that were designed to validate these features of the GaitEnable. Only healthy subjects were recruited, as these experiments investigated the capability of the system rather than its therapeutic effectiveness.

\subsection{Experiment Objectives}

The experiments described in this chapter investigate the following questions:

1. Does changing the desired mass reduce the effort required to move GaitEnable? By design, the enhanced admittance controller used on GaitEnable sacrifices impedance display fidelity to guarantee stability. Thus, the impedance manipulations, in addition to the torque limits of the servomotors, impose a fundamental limit on the minimum mass that GaitEnable can actually display. Thus, it can be taken for granted that GaitEnable may not be capable of accurately displaying a small mass. However, it is important to ensure that the behaviour of the device is consistent and predictable when the parameters of the admittance model are varied. Otherwise, tuning the admittance controller to relate the device's motion to therapy requirement would be impossible. Thus, a series of experiments were performed to determine whether 
changing the desired mass from a small value $(20 \mathrm{~kg})$ to a very small value $(4 \mathrm{~kg})$ resulted in the device feeling noticeably lighter.

2. Can a virtual assistance force applied through the admittance model allow a user to walk with less effort? GaitEnable should be capable of assisting frail individuals to walk, without directly commanding their motion, or forcing them to walk at a constant velocity. The feedforward inputs $F_{v}$ in the interaction model can be designed so that pushing forces are applied only when an individual initiates a step. However, due to the limitations discussed above, it cannot automatically be assumed that the desired virtual force will be manifested at the user-device interface. Thus, a series of experiments were performed to investigate whether virtual assistance forces of $10 \mathrm{~N}$ and $20 \mathrm{~N}$ reduced the overall force inputs required to move GaitEnable.

3. Does allowing lateral mobile base motions have a significant impact on a user's gait? Studies show that constraining lateral pelvis motions during rehabilitation therapy as many gait trainers with non-holonomic mobile bases do [72, 82, 163] - can lead to narrower steps, excessive trunk rotation, and other unnatural behaviours [47, 48, 51]. Thus, a series of experiments were performed to investigate whether omnidirectional mobile base motions result in reduced interaction forces and a more natural gait, in comparison to walking with lateral pelvis translations restricted.

4. Can the mobile base generate gait disturbances that invoke change-in-support reactions for balance training purposes? GaitEnable was designed with a powered omnidirectional mobile base so that user's can freely move in any direction during a changein-support reaction. A series of experiments were carried to investigate whether such change-in-support reactions are possible in practice.

5. How much easier is it to move GaitEnable with a powered mobile base versus with casters? The actuated mobile base should reduce the effort that user's need to exert to move GaitEnable. As such, an experiment was performed to quantify the reduction in the force inputs required to initiate and sustain the motion of GaitEnable with and 
without a powered base. Such a quantification provides a means for judging the benefits of using a powered base.

6. What effect does the passive linkage have? The passive linkage influences the humanrobot interaction because it creates a flexible connection between the user and GaitEnable, and alters the mobile base's ability to transmit forces to the user. As such, an experiment was performed to investigate how the force inputs required to move GaitEnable vary when walking with the passive linkage.

Subjects who participated in the walking experiments were requested to slowly walk in a straight line during all experiments. The choice to restrict the experiments to this simple motion was motivated by physiotherapist recommendations that suggested that the device would primarily be used to assist frail patients when they walk in a hallway or corridor. Thus, the experiments characterize the performance of the device during the type of activity that it is most likely to be used for.

The subject recruitment, screening, and informed consent procedures were reviewed by and received ethics clearance through the Carleton University Research Ethics Committee.

\subsection{Walking Experiments}

Four healthy males and three healthy females ranging in age between 24 and 30 were recruited to participate in the experiments. Participants were required to repeatedly perform a specific walking motion with GaitEnable. Various controller parameters were varied among repetitions so that the effects of changing mass properties, applying a virtual assistance force, and constraining lateral mobile base motions could be investigated. A repeated measures study design with all subjects tested at all different test conditions was used to reduce the total number of participants required for the study. 
The multi-lateral interface attached to the fixed arm supports of the mobile base described in Section 4.2.2, was used during the walking experiments. This ensured that the effects of the passive linkage did not influence the results. A separate experiment, discussed in Section 5.5, was carried out to investigate the effects of the passive linkage.

\subsubsection{Protocol}

All participants were instructed to walk with GaitEnable in the same way. Markers were placed on the floor to specify the sequence and spacing of the steps to be taken. A separation distance of $0.3 \mathrm{~m}$ was used between each subsequent marker, and the markers were placed such that the user walked in a straight line for approximately $3.3 \mathrm{~m}$. A short walking distance was used because of space limitations in the test facility, and because participants were requested to perform numerous repetitions of the experiment at different test conditions. Figure 5.1 shows image of the marker placement.

Participants were requested to walk slowly with a cadence of approximately 1 step per second, which resulted in an average walking speed of approximately $0.3 \mathrm{~m} / \mathrm{s}$. As noted, the slow walking speed and straight-line walking were meant to emulate the scenario of a patient walking down a hospital or clinic hallway. Participants were given the option to count out the 1 second intervals between the steps themselves, or to have the researcher count the intervals so that they could focus on walking at a consistent speed.

Three data collection test runs were performed with each participant at the following test conditions:

1. Walk with GaitEnable's desired mass specified as $20 \mathrm{~kg}$.

2. Walk with GaitEnable's desired mass specified as $4 \mathrm{~kg}$.

3. Assisted walking with GaitEnable's desired mass specified as $4 \mathrm{~kg}$ and a peak assistance force of $10 \mathrm{~N}$. 


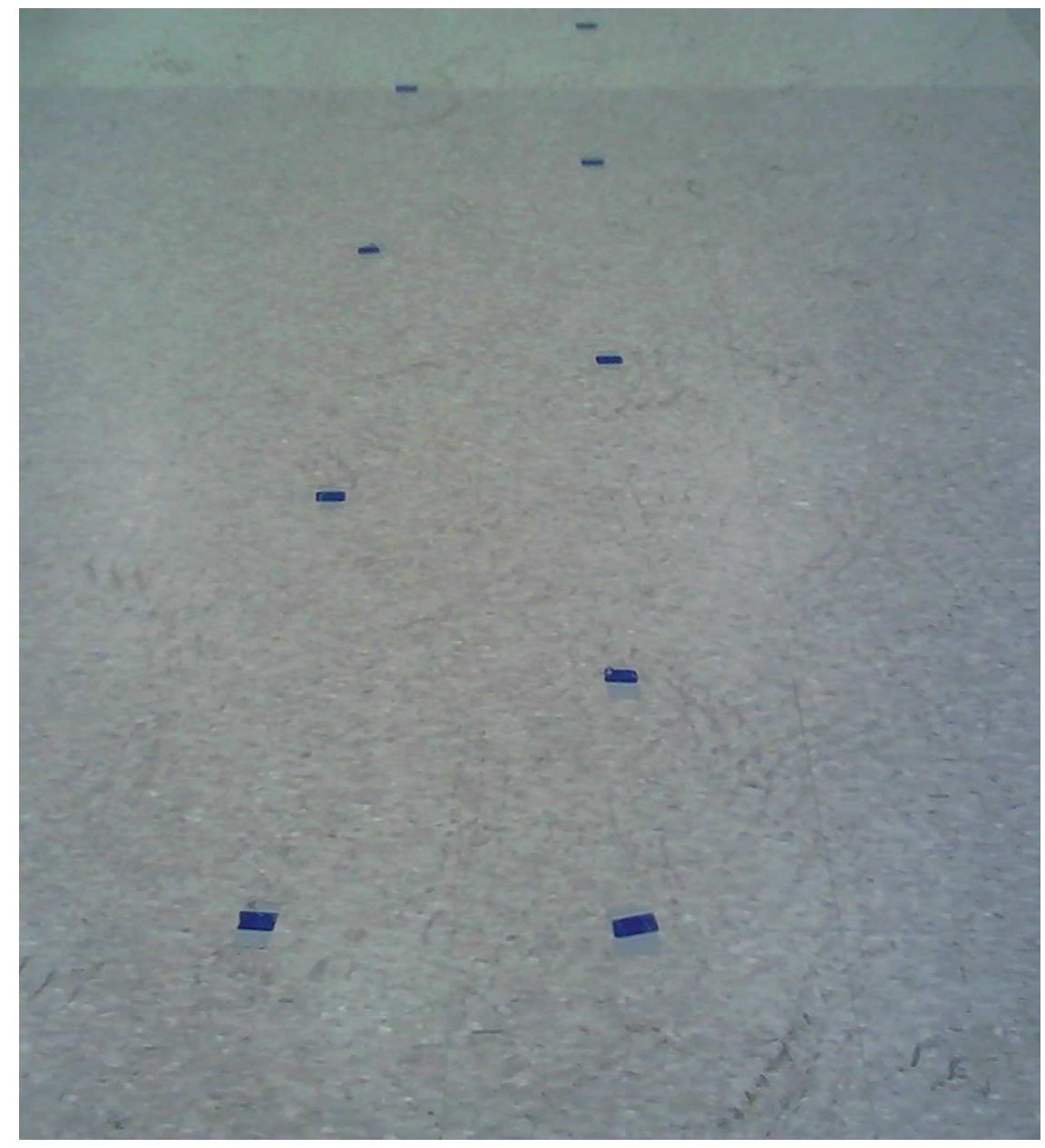

Figure 5.1: The markers placed on the floor to specify the sequence of steps to be taken.

4. Assisted walking with GaitEnable's desired mass specified as $4 \mathrm{~kg}$ and a peak assistance force of $20 \mathrm{~N}$.

5. Walking with lateral motion constrained, and GaitEnable's desired mass specified as $4 \mathrm{~kg}$.

The sequence of the test conditions was held fixed, and all participants were given the opportunity to repeat a test run if their cadence varied significantly form 1 step per second. 


\section{Controller Parameters}

The parameters of the acceleration limits were specified as $\psi_{w}=90 \frac{\mathrm{rad}}{\mathrm{s}^{2}}, \phi_{w}=40$, $\gamma_{w}=250 \frac{1}{s}, \eta_{w}=500 \frac{\mathrm{rad}}{\mathrm{s}^{2}}$, in all experiments. The $\mathrm{P}$ gains of the omniwheel proportional controllers were also held constant in all experiments, and were set to $K_{p, i}=3000 \frac{\mathrm{V}}{\mathrm{rad}}$. Identical acceleration limit and position controller gains could be used since identical servomotors were used to power each omniwheel.

\section{Changes in Virtual Impedance Model Parameters}

The parameters of the interaction model were specified as $m=20 \mathrm{~kg}, c=0 \mathrm{Nm} / \mathrm{s}, j=2$ $\mathrm{kgm}^{2}$, and $c_{\theta}=5 \mathrm{Nmrad} / \mathrm{s}^{2}$, and the error feedback parameter was set to $b_{w}=50$ in test condition 1 .

The parameters were changed to $m=4 \mathrm{~kg}, c=0 \mathrm{Nm} / \mathrm{s}, j=2 \mathrm{kgm}^{2}$, and $c_{\theta}=5$ $\mathrm{Nmrad} / \mathrm{s}^{2}$, and $b_{w}=208$ at all remaining test conditions. The error feedback parameter had to be increased since GaitEnable exhibited larger oscillations at the $4 \mathrm{~kg}$ mass setting.

The actual mass of GaitEnable is approximately $100 \mathrm{~kg}$. Thus, test conditions 2-5 required GaitEnable to display a $96 \%$ mass reduction.

\section{Assisted Walking with Virtual Pushing Forces}

Walking assistance in test conditions 3 and 4 was imparted to participants with the virtual force input $f_{y, v}$ designed as

$$
f_{y, v}= \begin{cases}0 & \text { if } f_{y, i}<0 \\ \delta\left(1-2\left(\frac{1}{1+e^{-0.75 f_{y, i}}}-0.5\right)\right) & \text { if } f_{y, i} \geq 0\end{cases}
$$




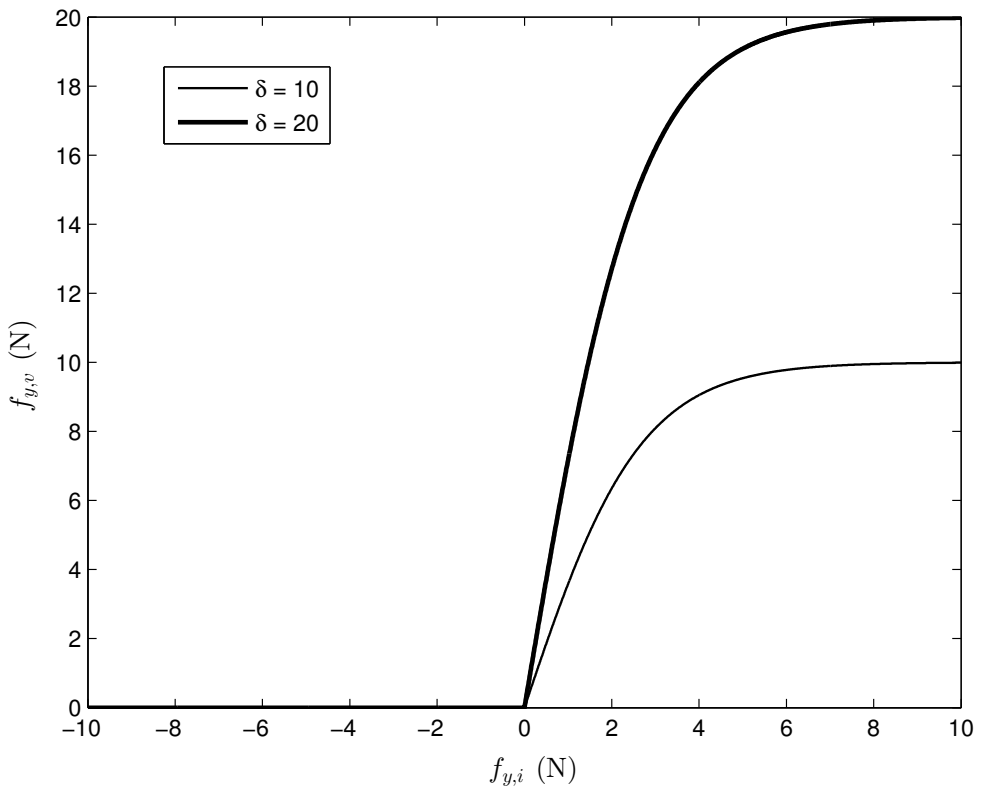

Figure 5.2: The relationship between assistance force and the force the user applies to the user-device interface.

where $\delta$ is the maximum value of the assisting force. Equation (5.1) is motivated by research that suggests that constant pushing/pulling forces at the pelvis are sufficient for significantly reducing the metabolic cost of walking [185]. The experiments in [185] were performed with a continuous pulling forces. However, participants indicated that continuous pushing forces felt uncomfortable because they constantly had to be resisted. This motivated the development of the assistance controller in (5.1) that selectively assists the user when the interaction force in the fore/aft direction, $f_{y, i}$, indicates that the user wishes to move forward. The assistance force is applied only in the forward walking direction, and has a nonlinear amplification at small positive values of $f_{y, i}$. The maximum assistance force is capped at the value of $\delta$ at large values of $f_{y, i}$. Users can comfortably stand still since the assistance force is nearly zero when the user applies small forces on the device. Figure 5.2 shows the relationship between the assistance force and the fore/aft interaction force. 


\section{Constrained Walking}

During the constrained motion tests of test condition 5, participants were constrained to walk in a straight line without the ability to rotate their pelvis or move laterally. This test condition approximated the behaviour of a differentially steered mobile base during straight-line walking. The motion constraints were implemented by setting $f_{x, i}=\tau_{\theta}=0$ and $c=c_{\theta}=100$ in the interaction model (4.2).

\subsubsection{Data Collection}

Measurements of the interaction forces and moments, omniwheel orientations, and the velocity of the reference point were recorded during all experiments. The velocity of the reference point was estimated from omniwheel orientation data as follows:

1. Encoder position data were numerically differentiated to estimate each omniwheel's angular velocity. The velocity estimate was filtered with a digital first-order filter with a $30 \mathrm{~Hz}$ bandwidth.

2. The filtered velocity estimates were multiplied by the mobile base Jacobian to estimate $\dot{x}, \dot{y}$, and $\dot{\theta}$.

3. The acceleration components $\ddot{x}, \ddot{y}$, and $\ddot{\theta}$ were calculated by differentiating $\dot{x}, \dot{y}$, and $\dot{\theta}$. A digital first-order filter with a $30 \mathrm{~Hz}$ bandwidth was used to filter the differentiated signals.

\subsubsection{Characterizing Change in Effort}

The work done on the device by the user to move it from its initial position to its final position, $W$, was used to characterize effort. $W$ was calculated as follows: 


$$
W=\int_{0}^{T} f_{x, i} \dot{x}+f_{y, i} \dot{y}+\tau_{\theta} \dot{\theta} d t
$$

where $\dot{x}, \dot{y}$, and $\dot{\theta}$ denote the velocity of the reference point, and $T$ denotes the duration of a single $3.3 \mathrm{~m}$ walk from the start position to the final position. The integrand of (5.2) denotes the instantaneous mechanical power that the user contributes to generate the motion. As the initial and final positions of the walking motion were held constant, and since participants were requested to walk at the same speed at each test condition, the change in $W$ among test conditions indicates how participants' effort levels varied among test conditions. A decrease in $W$ among test conditions indicates that GaitEnable was easier to move at the new test condition. Conversely, an increase in $W$ denotes an increase in user effort, and the average force input required to move GaitEnable. The values of $W$ calculated from the final test runs that subjects performed at each of the 5 test conditions was used in the analysis.

GaitEnable's effective mass display was also calculated to asses impedance display fidelity. The following relations were used for this purpose:

$$
\begin{aligned}
& \hat{m}_{x}=\sqrt{\frac{\frac{1}{n} \sum_{k=0}^{n} f_{x, i}^{2}}{\frac{1}{n} \sum_{i=0}^{n} \ddot{x}^{2}} ;} \\
& \hat{m}_{y}=\sqrt{\frac{\frac{1}{n} \sum_{k=0}^{n} f_{y, i}^{2}}{\frac{1}{n} \sum_{i=0}^{n} \ddot{y}^{2}}} ;
\end{aligned}
$$


where, $\hat{m}_{x}$, is the effective mass displayed in the forward direction and $\hat{m}_{y}$ is the effective mass displayed in the lateral direction. The bracketed quantities in the numerators of (5.3) and (5.4) represent the RMS force input that a participant applied to GaitEnable during a test run. The RMS force divided by the RMS acceleration provides an estimate of the effective mass that GaitEnable displayed during a test run.

For a perfect impedance display, $\hat{m}_{y}=\hat{m}_{x}=m$. However, both velocity estimation errors and the effects of the digital filter used to filter $\ddot{x}$ and $\ddot{y}$ introduce errors in the calculations of the effective masses. Thus, $\hat{m}_{x}$ and $\hat{m}_{y}$ only provide an approximate measure of the average mass that GaitEnable displayed.

The effects of the virtual assistance force are deliberately excluded in the calculation of $\hat{m}_{y}$. Thus, changes in $\hat{m}_{y}$ due to the use of (5.1), provide a means for confirming if the virtual assistance forces had an effect on making the device feel lighter and easier to move.

The values of $\hat{m}_{x}$ and $\hat{m}_{y}$ reported in the subsequent sections denote the mean values calculated over all participants and all test runs. As each of the seven subjects performed three data collection test runs, a total of 21 samples were used to calculate the averages of $\hat{m}_{x}$ and $\hat{m}_{y}$ at each test condition.

\subsubsection{Results}

\section{Velocity Estimation Errors}

As omniwheel slip limits the accuracy of the reference point velocity estimates calculated from encoder data, an experiment was performed to confirm whether omniwheel slip could introduce unacceptably large velocity estimation errors. During the experiment, an 
Optotrack Certus Motion capture system was used to track the position trajectories of two optical markers placed at opposite ends of the mobile base's frame. The motion capture system uses active markers, multiple cameras, and a triangulation algorithm to estimate marker positions in three dimensions with respect to a camera-fixed frame. Based on the manufacturer's specifications, the position trajectories of these markers should be accurate to within $0.1 \mathrm{~mm}$.

A pre-calibrated probe supplied by the manufacturer was used to measure the fixed offset between the reference point and one of the markers. Thus, the position trajectory of the reference point was estimated as the sum of the fixed offset and the position trajectory of one of the markers. The velocity components $\dot{x}, \dot{y}$, and $\dot{\theta}$ ) were calculated by differentiating the reference point trajectory. As marker positions were collected at a frequency of $100 \mathrm{~Hz}$, encoder and interaction force data collected from GaitEnable was downsampled by a factor of 20 prior to being used in the analysis. In order to synchronize the data from both systems, a signal generated from GaitEnable's data acquisition card triggered the motion capture system.

Results comparing the motion capture velocity measurements and the encoder velocity estimates are shown in Figure 5.3. This figure demonstrates a general agreement among the both measurement types. However, the encoder-based estimates deviate from the motion capture measurements at the zero-crossings of acceleration (e.g., at the velocity peaks between 5 and 15 seconds in Figure 5.3a). While the peak deviations were large and nearly $16 \%$ of the maximum fore/aft velocity in Figure 5.3a, for example, Table 5.1 shows that the relative difference in the values of $W$, the measure of interest, was a negligible $3.2 \%$. As such, the encoder-based measurements were deemed acceptable.

\section{Sample Results from Participant 1}

Selected results from 1 participant are presented below as they are representative of the type of data collected from the experiments. Figure 5.4 shows the mobile base velocity and 


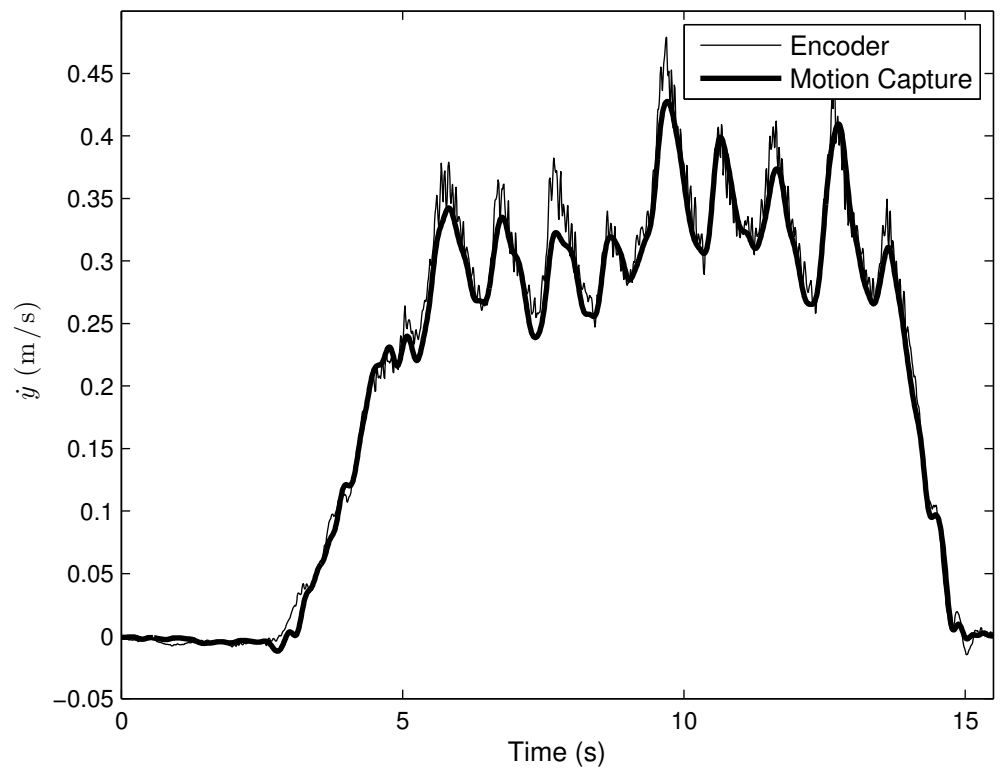

(a) Fore/aft velocity

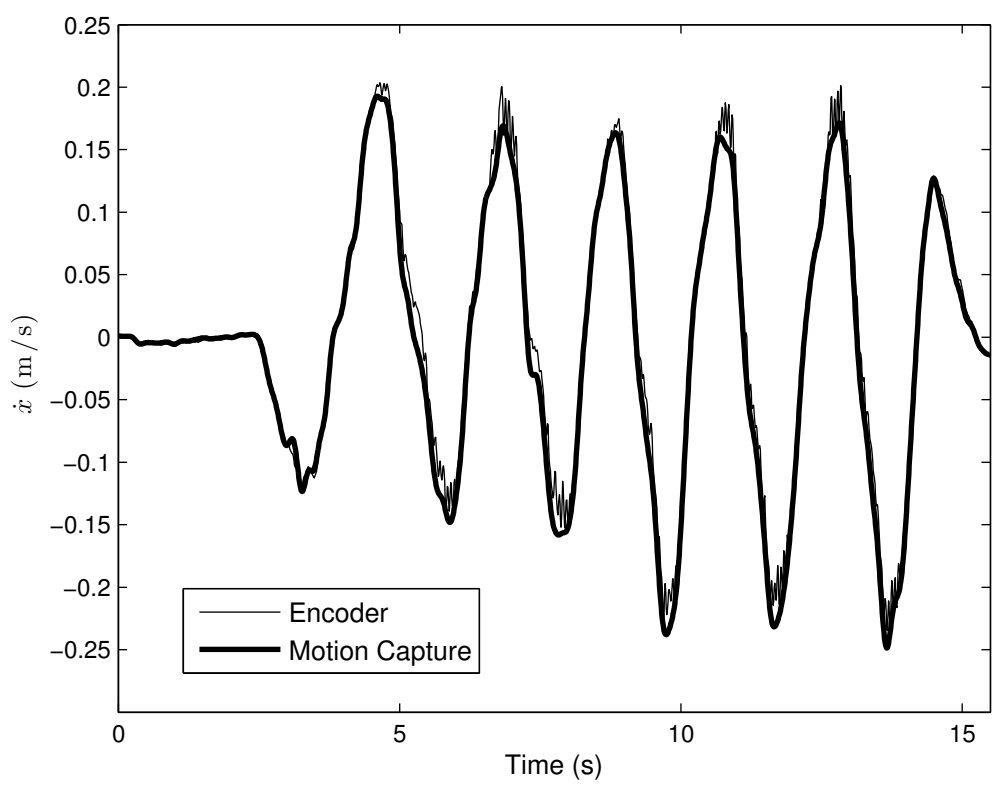

(b) Lateral velocity

Figure 5.3: Comparing the reference point velocity estimated from the encoders to the velocity estimated from the motion capture system. Detailed captions can be found on the following page. 


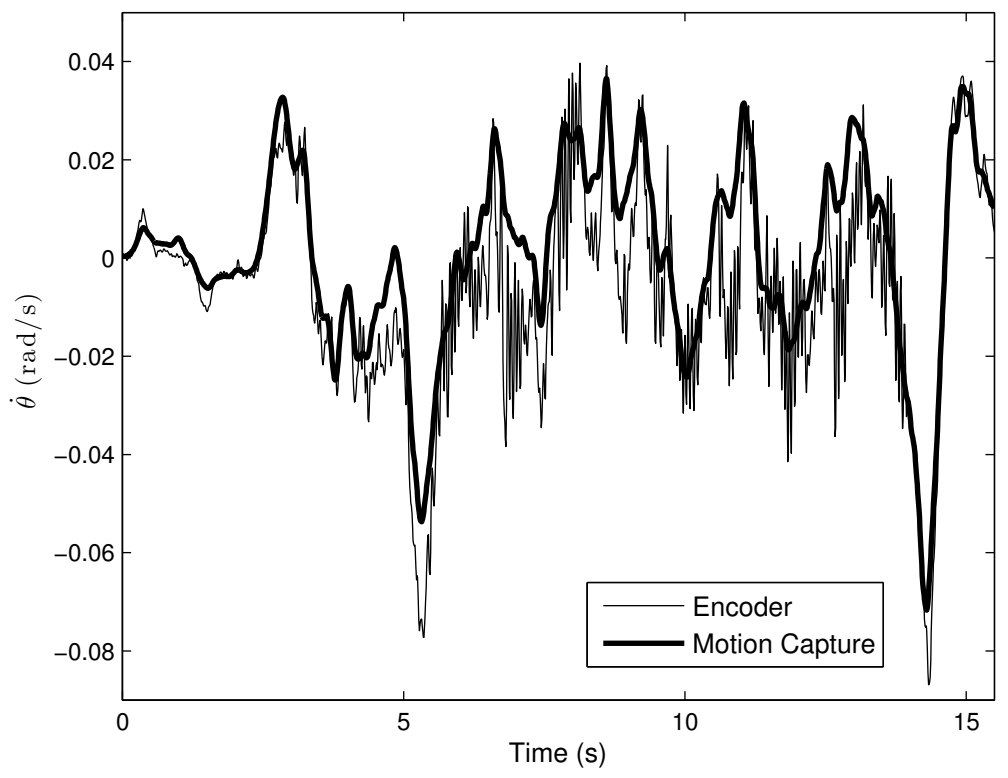

(c) Angular Velocity

Figure 5.3: Comparing the reference point velocity estimated from the encoders to the velocity measured from the motion capture system. (a) Fore/aft velocity. (b) Lateral velocity. (c) Angular Velocity. Encoder-based velocity estimates show the greatest deviation during acceleration reversals, and show the poorest comparison for the angular velocity of the mobile base. 
Table 5.1: Comparing the root mean square values of the encoder- and motion capture system-based reference point velocity estimates

\begin{tabular}{|c|c|}
\hline & $W(\mathrm{~J})$ \\
\hline Encoder & 34.5 \\
Motion capture system & 33.4 \\
\hline Relative difference & $3.2 \%$ \\
\hline
\end{tabular}

interaction force data from test conditions 1 and 4 for participant 1 . The non-zero lateral and angular velocities of the mobile base in Figures 5.4c and 5.4e confirm that the mobile base is capable of omnidirectional motion. The non-zero lateral velocity also confirms that the mobile base is capable of moving sideways, a crucial body motion $[47,48,51]$ that differentially-steered mobile bases inhibit.

There are three general features in the data in Figure 5.4 worth noting:

1. Lateral pelvis velocity is approximately sinusoidal

2. Lateral pelvis velocity has approximately half the frequency of the fore/aft pelvis velocity

3. Fore/aft pelvis excursions during steady walking have a smaller amplitude than the corresponding lateral pelvis excursions

All three features are important because they are qualitatively consistent with the pelvis data for normal gait described in $[97,98]$. As such, these features suggest that GaitEnable's omnidirectional mobile base is effective at preserving some key features of normal pelvis motion during slow walking.

\section{Assessing the Significance of the Changes in $W$ Between Test Conditions}

The work required to move the device $W$ was subjected to a repeated measures analysis of variance having five test conditions. Using a Greenhouse-Geisser correction of 0.565 , the 


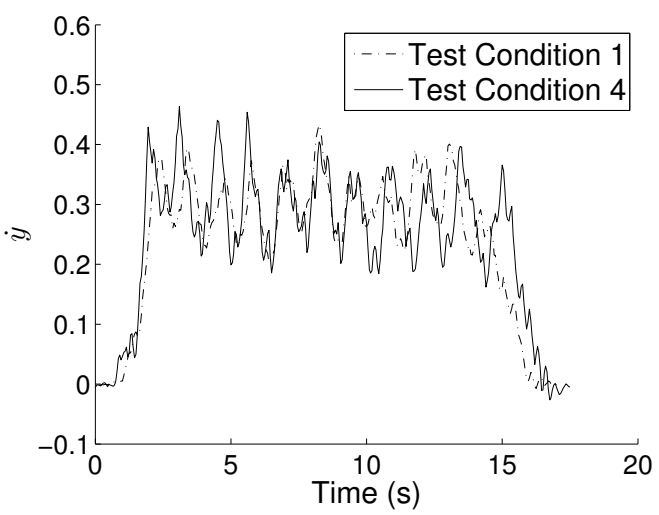

(a) Fore/aft velocity

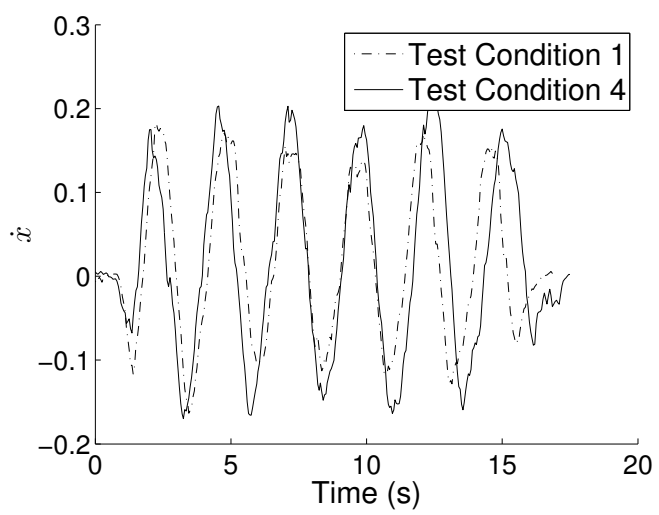

(c) Lateral velocity

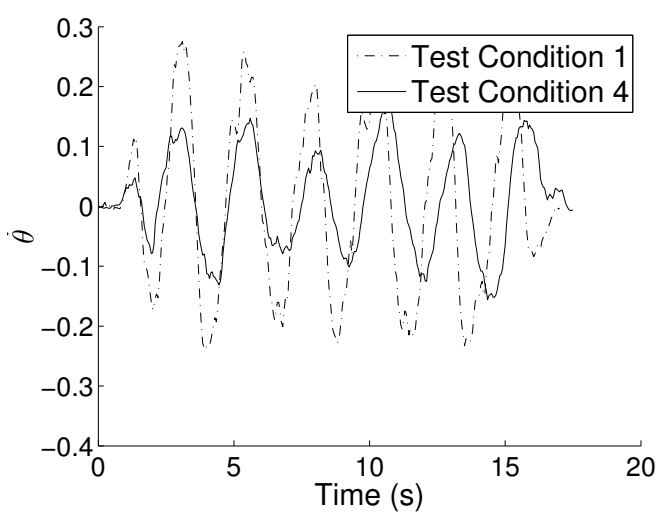

(e) Angular velocity

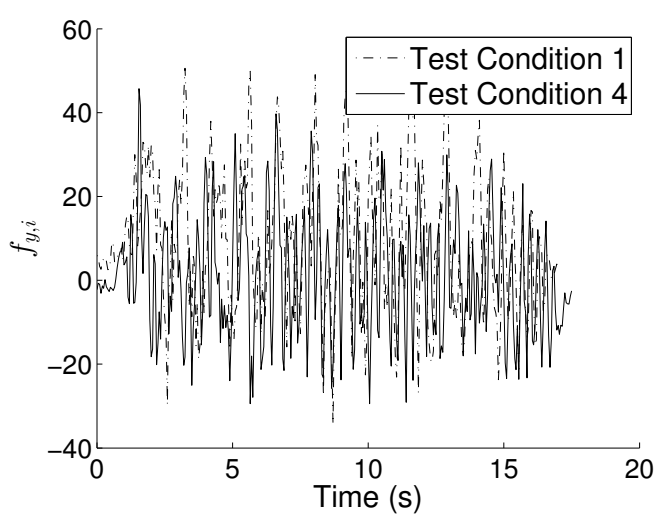

(b) Fore/aft interaction force $f_{y, i}$

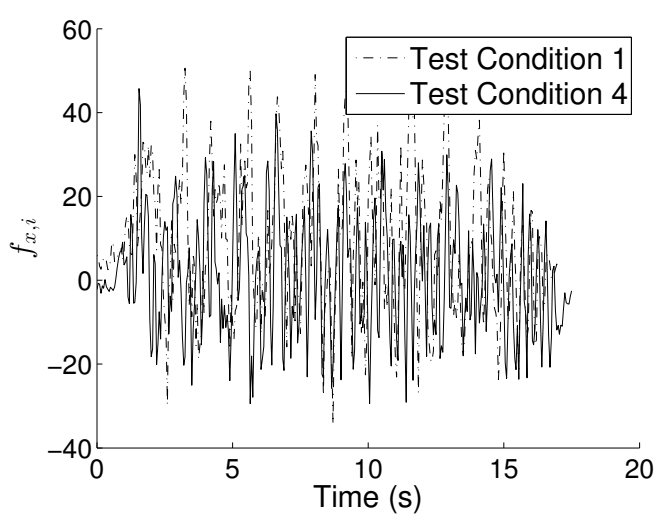

(d) Lateral interaction force $f_{x, i}$

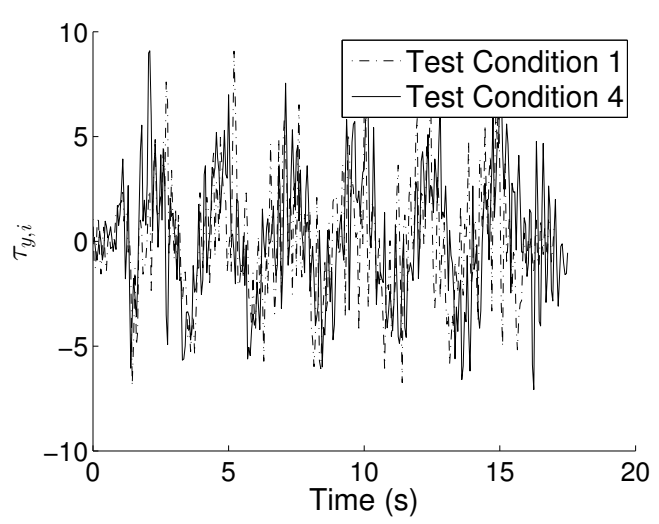

(f) Interaction torque $\tau_{\theta}$

Figure 5.4: Sample results from walking experiments from one participant. (a) Fore/aft velocity. (b) Fore/aft interaction force. (c) Lateral velocity. (d) Lateral interaction force. (e) Angular velocity. (f) Interaction torque. The non-zero lateral and angular velocities demonstrate the omnidirectional motion capability of the device. 


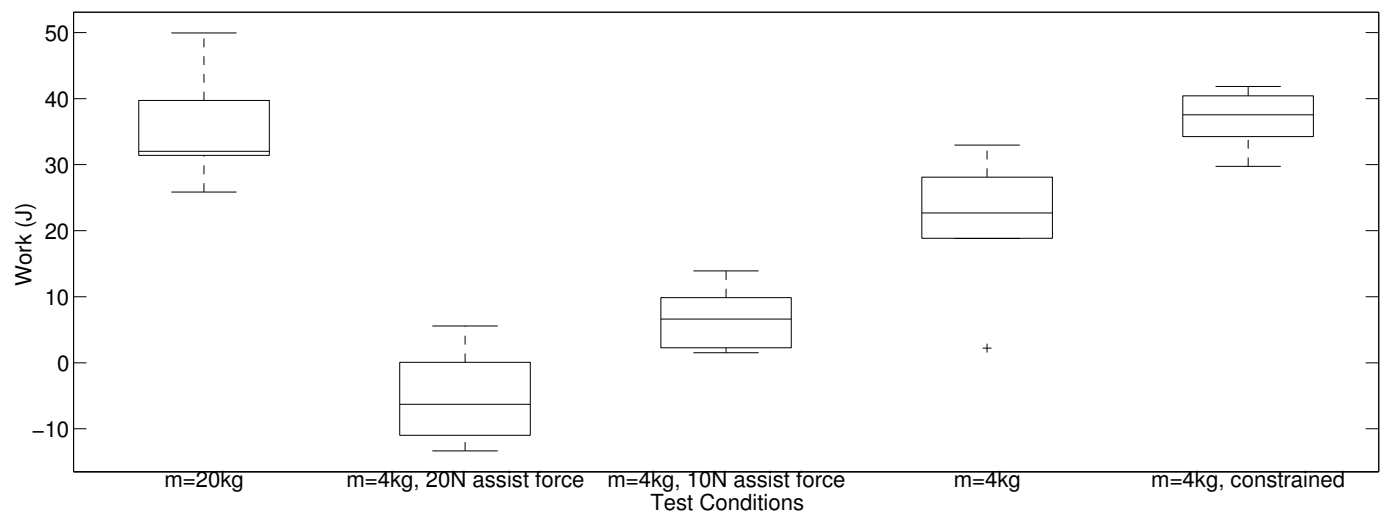

Figure 5.5: A box plot describing the variation of $W$ at the different test conditions.

effect of test conditions was found to be significant with an $\mathrm{F}$ ratio $F(2.3,13.6)=61.4$, $p<0.0001$. Thus, changing the desired mass, applying an assistance force, and constraining the user's motion had a statistically significant effect on $W$.

Figure 5.5 shows how $W$ varied between test conditions; negative values of $W$ imply that the mean interaction forces and torque were negative, and that participants were primarily resisting the device. This figure confirms that reducing the desired mass, and applying assistance forces reduce $W$. Also, constraining pelvis motion increases $W$ at a given mass setting. Overall, this trend demonstrates that GaitEnable's behaviour was consistent with the parameter changes - the device was easier to move when the desired mass was reduced, and when the assistance forces were applied, but harder to move when unnatural constraints on participants' pelvis motions were enforced. Additional details and results are provided in the sections below. 


\section{The Effects of Changing GaitEnable's Desired Mass}

Table 5.2 shows the effective mass and inertia data from the $m=20 \mathrm{~kg}$ and $m=4 \mathrm{~kg}$ test conditions. These results show that the effective masses and inertias that GaitEnable displayed were significantly higher than the desired values. This result is not entirely unexpected. As noted in Section 5.1, both the impedance manipulations and the torque limits of the servomotors impose a limit on the minimum mass that GaitEnable can display. In fact, the real motivation for performing this experiment was to investigate whether changing the desired mass resulted in consistent changes in the behaviour of GaitEnable. The results in Table 2 confirm this, as they show that the effective masses displayed by GaitEnable were reduced by nearly $29 \%$ at test condition 2 .

In addition, the results in 5.2 show that GaitEnable feels lighter when moving sideways instead of front and back. This result is not unexpected and reflects the effects of GaitEnable's direction-dependent acceleration capability. Due to the orientation of the omniwheels, GaitEnable can generate peak traction forces of $360 \mathrm{~N}$ and $613 \mathrm{~N}$ in the fore/aft and lateral directions, respectively. Thus, the fact $\hat{m}_{x}$ was less than $\hat{m}_{y}$ at both test conditions reflects GaitEnable's improved ability to accelerate laterally.

Table 5.2: The effective masses and inertias displayed by GaitEnable at 2 different desired mass settings

\begin{tabular}{|c|c|c|}
\hline & $\begin{array}{c}\hat{m}_{x}(\mathrm{~kg}) \\
\text { mean (s.d.) }\end{array}$ & $\begin{array}{c}\hat{m}_{y}(\mathrm{~kg}) \\
\text { mean (s.d.) }\end{array}$ \\
\hline$m=20, j=2$ - Test Condition 1 & $39.1(2.3)$ & $53.3(1.5)$ \\
$m=4, j=2-$ Test Condition 2 & $32.7(3.2)$ & $42.4(2.0)$ \\
\hline
\end{tabular}

Omniwheel vibrations may also have contributed to the higher than expected mass display. These vibrations are most apparent at low speeds, and generate oscillatory interaction forces with peak-to-peak amplitudes greater than 10 N. Results from a simple experiment during which a participant was requested to slowly push GaitEnable backwards 
presents an example of this behavior. As can be seen in Figure 5.6, moving the mobile base at a slow, constant speed is challenging because of the omniwheel-induced vibrations. The impedance manipulations are unable to attenuate these oscillations as they are caused by the fluctuations of the ground contact point during omniwheel roller transitions, a physical phenomenon that neither the impedance manipulations nor the actuators can compensate. These oscillations are undesirable because they increase the RMS force input required to sustain the motion of the device. As such, they also cause GaitEnable to feel heavier.

Inaccurate sensor readings may have also contributed to the inaccurate impedance display observed in the experiments. The pelvis harness used to constrain the user to the device has a mass of $6.8 \mathrm{~kg}$. As its CoM is offset from the force sensor, the measured interaction forces/torques are corrupted by the inertial forces/torques of the pelvis harness. As the peak mobile base accelerations observed during the experiments were under 2 $\mathrm{m} / \mathrm{s}^{2}$, the inertial loads of the harness should have contributed to peak interaction force estimation errors on the order of $10 \mathrm{~N}$.

\section{The Effect of the Assistance Force}

Therapists using passive walking support systems such as a platform walker or mobile patient lift must manually push the device to assist a patient in taking steps. GaitEnable's mobile base has the potential to automatically provide the propulsion force required to initiate and sustain the patient's gait. This feature of GaitEnable was investigated in test conditions 3 and 4. As described in Section 5.2.1, stepping assistance was emulated via the feedforward input $f_{y, v}$. Assistance forces of $10 \mathrm{~N}$ and $20 \mathrm{~N}$ were specified for test conditions 3 and 4, respectively. Test condition 2 was performed without any assistance force and otherwise identical controller parameters. Thus, it provides an appropriate comparison point for determining the effects of adding the assistance forces. Table 5.3 shows the mean RMS values of $f_{y, i}$, and $\hat{m}_{y}$ for the three test conditions.

The results in Table 2 confirm that the RMS force inputs required to move GaitEnable 


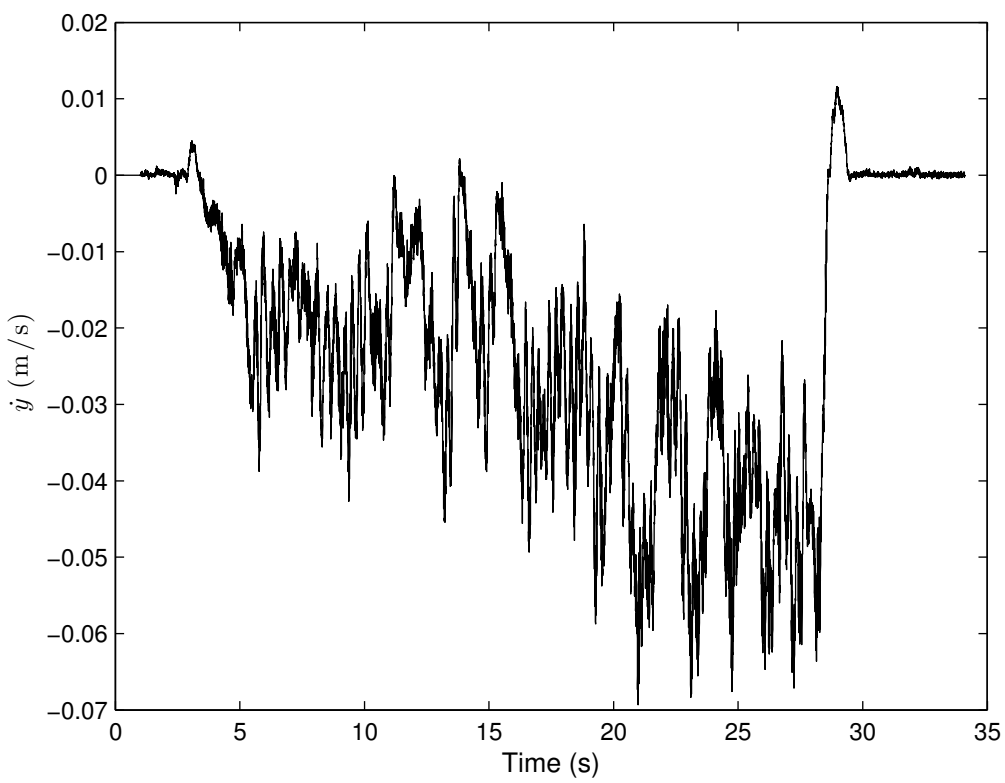

(a) Fore/aft velocity

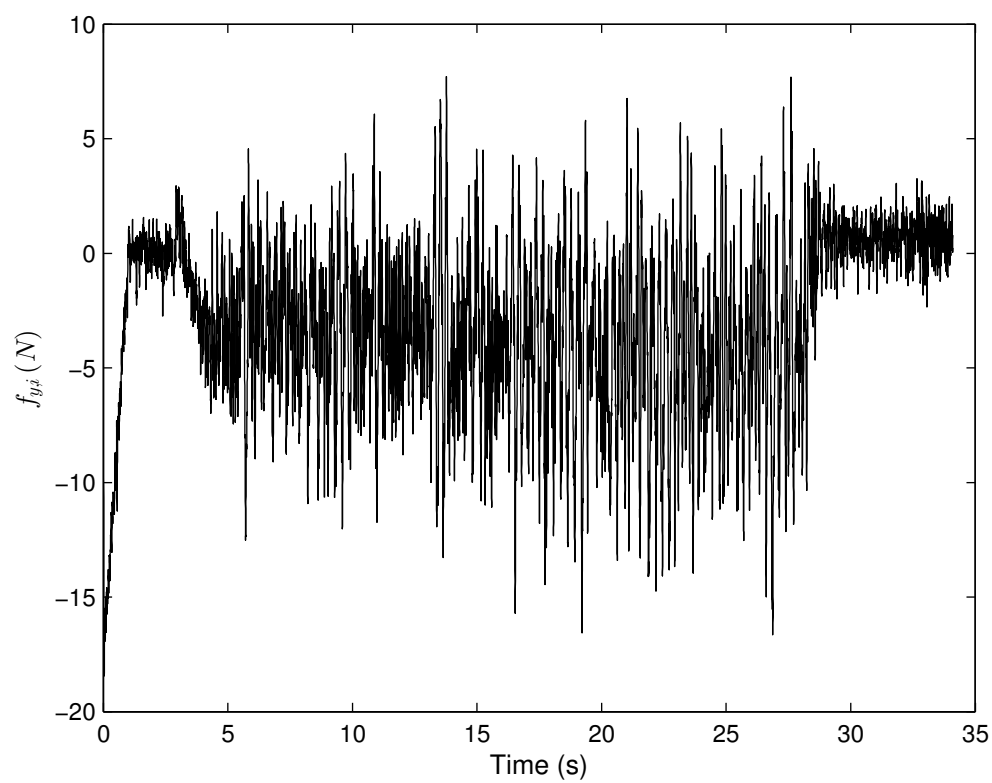

(b) Fore/aft interaction force $f_{y, i}$

Figure 5.6: Low speed vibrations generated by omniwheels. (a) Fore/aft velocity. (b) Fore/aft interaction force. The fluctuation of the ground contact point during omniwheel roller transitions generates mobile base vibrations that interfere with the user-device interaction. These vibrations have a peak-to-peak amplitude on the order of $10 \mathrm{~N}$. 
Table 5.3: Changes in the RMS interaction forces and moments as a result of applying assistance forces through the admittance model.

\begin{tabular}{|c|c|c|}
\hline & $\begin{array}{c}f_{y, i}(\mathrm{~N}) \\
\text { mean (s.d.) }\end{array}$ & $\begin{array}{c}\hat{m}_{y}(\mathrm{~kg}) \\
\text { mean (s.d.) }\end{array}$ \\
\hline$m=4 \mathrm{~kg}$ and No Assistance - Test Condition 2 & $20.6(1.6)$ & $42.4(2.0)$ \\
$m=4 \mathrm{~kg}$ and $10 \mathrm{~N}$ Assistance - Test Condition 3 & $16.2(2.1)$ & $33.6(2.4)$ \\
$m=4 \mathrm{~kg}$ and $20 \mathrm{~N}$ Assistance - Test Condition 4 & $16.4(1.8)$ & $29.0(1.4)$ \\
\hline
\end{tabular}

decreased by nearly $20 \%$ when the assistance forces were applied. Interestingly, the difference in $f_{y, i}$ as the assistance force was changed from $10 \mathrm{~N}$ to $20 \mathrm{~N}$ was negligible. However, the decrease in $\hat{m}_{y}$ between test conditions 3 and 4 implies that the average acceleration of GaitEnable increased when the assistance force was increased from $10 \mathrm{~N}$ to $20 \mathrm{~N}$. In other words, increasing the assistance force $10 \mathrm{~N}$ to $20 \mathrm{~N}$ allowed users to accelerate GaitEnable more rapidly with smaller force inputs. Thus, the results confirm that the virtual assistance forces are capable of reducing the mean propulsion forces required to move GaitEnable.

\section{Comparing Omnidirectional and Constrained Mobile Base Motions}

Most actuated mobile gait trainers employ non-holonomic differential steering systems that constrain a patient's lateral pelvis translation $[72,73,162]$. Test condition 5 was designed to emulate the behaviour of a differentially-steered mobile base during straight line walking. During this test condition, the reference trajectory was generated by setting $f_{x, i}$ and $\tau_{\theta}$ to zero in (4.2). This restricted the mobile base's lateral translations and rotations since $\ddot{x}_{r}$ and $\ddot{\tau}_{\theta}$ were restricted be zero for the duration of the test run. Thus, subjects could walk forwards easily, but were restricted from rotating the device or translating laterally.

The RMS values of the $f_{x, i}$ and $\tau_{\theta}$ were also calculated and compared. These values, and the corresponding averaged RMS lateral and angular velocities from test conditions 2 and 5 are reported in Table 5.4. These results show that the RMS values $\dot{x}$ and $\dot{\theta}$ were reduced by a factor of 10 when the mobile base's motion was constrained in test condition 5. This confirms that the mobile base was effective at constraining participants' lateral 
translation and rotation.

Table 5.4: Changes in the RMS interaction forces and moments between omnidirectional and constrained walking.

\begin{tabular}{|c|c|c|c|c|}
\hline & $\begin{array}{c}f_{x, i}(\mathrm{~N}) \\
\text { mean (s.d.) }\end{array}$ & $\begin{array}{c}\tau_{\theta}(\mathrm{Nm}) \\
\text { mean (s.d.) }\end{array}$ & $\begin{array}{c}\dot{x}(\mathrm{Nm}) \\
\text { mean (s.d.) }\end{array}$ & $\begin{array}{c}\dot{\theta}(\mathrm{rad} / \mathrm{s}) \\
\text { mean (s.d.) }\end{array}$ \\
\hline Test Condition 2 & $12.0(1.3)$ & $3.6(0.5)$ & $0.10(0.02)$ & $0.08(0.02)$ \\
Test Condition 5 & $26.4(8.0)$ & $5.6(2.0)$ & $0.01(0.005)$ & $0.008(0.002)$ \\
\hline
\end{tabular}

Table 5.4 also shows that there was a noticeable change in pelvis kinetics between test conditions 1 and 2. The average RMS values of $f_{x, i}$ and $\tau_{\theta}$ increased by $220 \%$ and $150 \%$, respectively, when participants were constrained to walk in a straight line. What these results do not portray is how much participants deviated from a natural walking pattern during test condition 5. All participants adopted a gait similar to a penguin's waddle; their inability to translate laterally forced them to use excessive trunk rotations as a means of supporting their body-weight during single-stance. This observation is consistent with the results presented in $[47,48,51]$ wherein robots or mechanical fixtures were similarly used to restrict an individual's pelvis translations. Though test condition 5 does not fully emulate the motion of a differentially-steered mobile base, the results from this experiment illustrate the type of irregular gait patterns that could be observed with mobile gait trainers designed with non-holonomic drive systems.

Table 5.4 also shows that the standard deviations of $f_{x, i}$ and $\tau_{\theta}$ are significantly larger at test condition 5. This result is consistent with the different compensation techniques observed among participants. Generally speaking, taller participants were capable of following the sequence of steps more easily and tended not to rotate their trunk quite as much. Also, while all participants maintained their stride length by following the marked sequence of steps on the floor, some chose to keep their feet closer together while walking. Those who kept their feet closer together were also observed to have less trunk rotation. Finally some participants may have worn the padded harness more loosely 
than others. This would have allowed their pelvis to have larger free play relative to the harness more, and could have contributed to a reduced resistance to rotation. Thus, all factors combined explain why more variability in $f_{x, i}$ and $\tau_{\theta}$ was observed at test condition 5 .

Overall, the comparison between test condition 2 and 5 confirms that an omnidirectional mobile base reduces the forces and moments generated at a user's pelvis, and that omnidirectional motion allows users to walk more naturally while strapped into GaitEnable.

\subsection{Generating Gait Perturbations via the Admittance Model}

Perturbation-based balance training, an approach wherein a mechanism or device actively perturbs a patient during their training, has grown in popularity in recent years $[57,165-167]$. Proponents of this training note that actively perturbing patients during their training increases training specificity, and may allow patients to better train compensatory postural reactions. GaitEnable is well-suited for this training because its interaction control system can be programmed to display fore/aft, lateral, and moment disturbances via the feedforward inputs $f_{y, v}, f_{x, v}$, and $\tau_{\theta}$, and because its capability for omnidirectional motion allows user to practice change-in-support reactions.

GaitEnable's ability to generate perturbations and evoke change-in-support reactions was tested in an experiment with seven healthy subjects. During the experiment, participants were asked to stand still with both feet on the ground and to resist a $100 \mathrm{~N}$ sideways push to the right from GaitEnable. Since the perturbation was large, participants were warned of the impending disturbance 10 seconds before its application.

All seven participants that performed the experiment exhibited a change-in-support reaction. Peak interaction forces in excess of $-100 \mathrm{~N}$ were seen from all subjects in response 
to the disturbance. Given that a positive disturbance of $100 \mathrm{~N}$ was applied, this confirms that the magnitude of the disturbance imparted to the user was consistent with the desired disturbance value of $100 \mathrm{~N}$. However, the specific reactions among participants varied. Some participants took a step to the side and stabilized, while others simply lifted one leg off the ground and leaned to the right. An example of each of these reactions is shown in Figure 5.7. As illustrated in Figure 5.7a, the act of taking a step to the side generally resulted in a larger mobile base velocity and a longer settling time. Also, the interaction force data from participants who leaned to one side exhibited a well-defined negative peak similar to the one shown in Figure 5.7b.

Participants also performed similar experiments where they were required to resist a $100 \mathrm{~N}$ fore/aft disturbance and a moment disturbance of $80 \mathrm{Nm}$. Both the fore/aft and moment disturbances were found to be less effective at generating change-in-support reactions. Fore/aft disturbances were easier to resist since participants simply had to lean forward to stabilize themselves. Only one participant reacted by taking a step forward to stabilize. Moment disturbances were found to be less effective because the soft harness slipped around the participants' waist. No participant reacted with a change-in-support reaction to the moment disturbance.

As all participants were made aware of the disturbance type and duration, it is not surprising that the fore/aft and moment disturbances were found to be less effective. Due to safety concerns, it was not possible to test how participants might react to unexpected disturbances. However, it is likely that smaller disturbance magnitudes may be sufficient to destabilize healthy subjects, and patients, when the disturbances are unexpected and applied mid-step. 


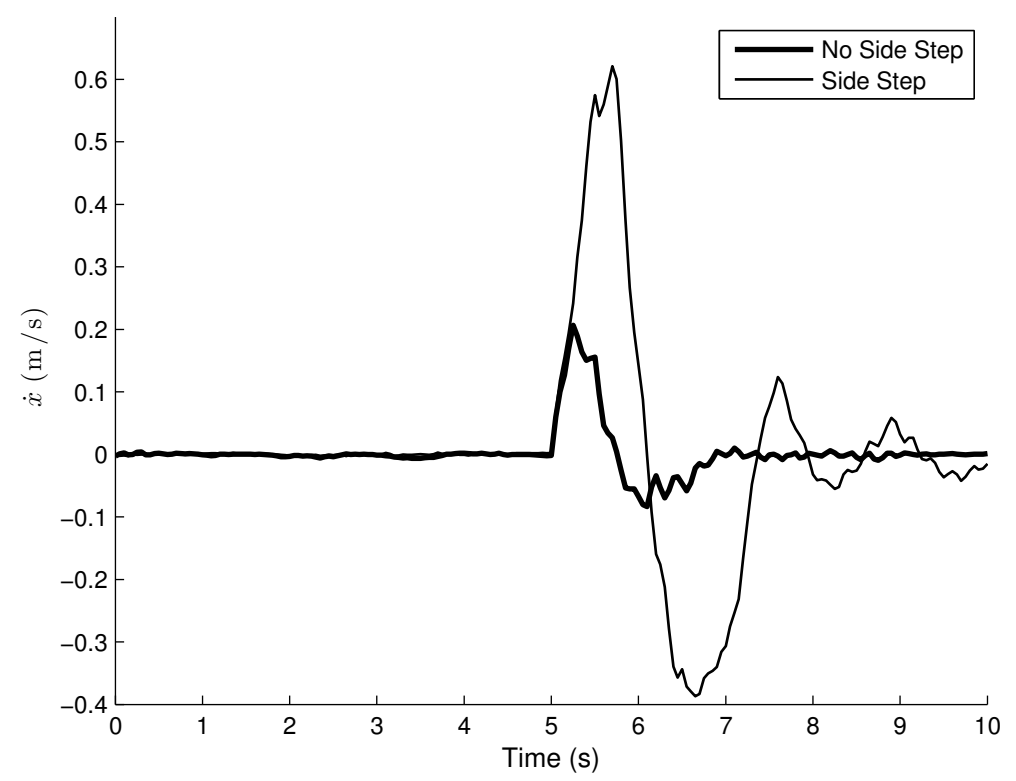

(a) Fore/aft velocity

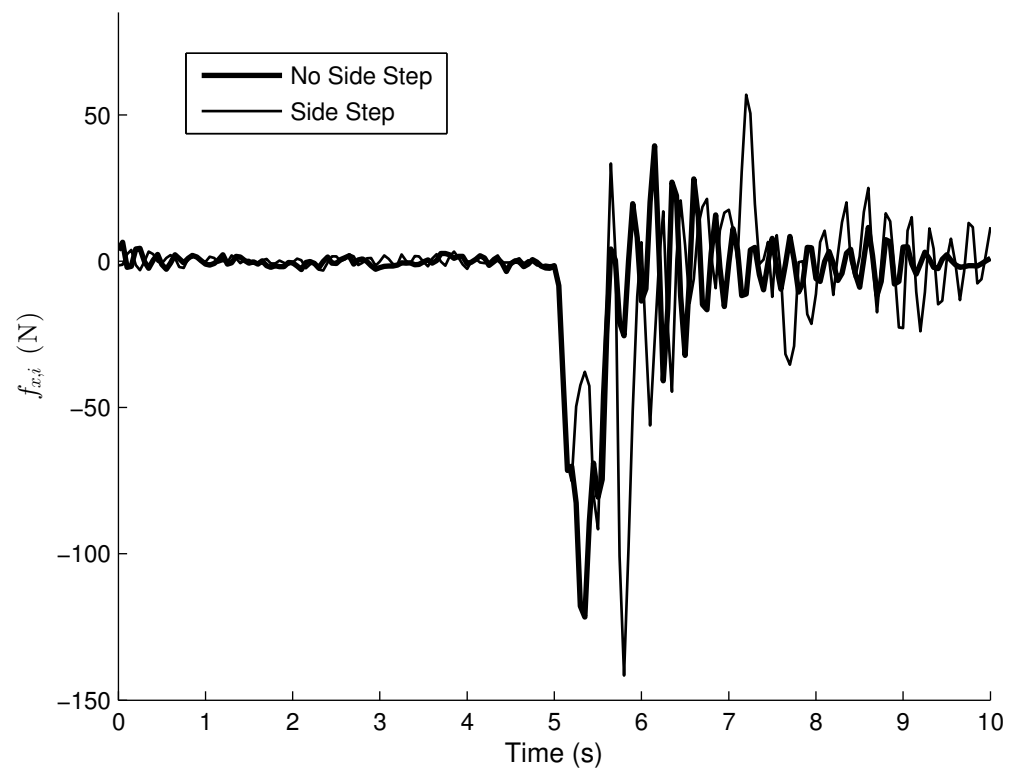

(b) Fore/aft interaction force $f_{y, i}$

Figure 5.7: Different reactions to lateral disturbances from GaitEnable. (a) Fore/aft velocity. (b) Fore/aft interaction force. Participants with better balance control lifted one leg and leaned to the side without allowing GaitEnable to move a large amount. Other participants had to take a step to the side and stabilize afterwards. This reaction strategy resulted in larger lateral velocities and a longer settling time of the lateral forces. 


\subsection{Comparing the Force Input Required with and without a Powered Base}

One participant was requested to return for an experiment designed to investigate the benefit of GaitEnable's actuated mobile base. During the experiment, casters were temporarily added to GaitEnable's mobile base, and the omniwheels were raised to ensure that they did not make contact with the ground. As no equipment was removed from GaitEnable, the user was effectively required to propel a passive walker weighing $100 \mathrm{~kg}$.

The participant was requested to perform 10 repetitions of walking motion used during test condition 4. As the omniwheels and servomotors were raised off the ground, mobile base velocity and acceleration data were not available.

Figure 5.8 compares the fore/aft interaction force with casters versus with the powered mobile base active. The figure shows a significant reduction in the peak and mean force inputs required to move GaitEnable when the powered base is used. The RMS values of $f_{y, i}$ and the average push-off force required to initiate motion are tabulated in Table 5.5. These results show that both the average push-off force and $f_{y, i}$ are reduced by over $300 \%$ with the powered base. Since brushed DC motors with a large gear ratio and high inertia were used, additional improvements may be possible if brushless motors with a lower inertia, and higher torque capacity are used in future designs. As other gait trainers were not available for testing, GaitEnable could not be compared to lighter devices like the BMT or Rifton Tram [190]. However, these results do demonstrate that the mobile base significantly reduces the effort that a user needs to exert move GaitEnable. 
Table 5.5: Comparing walking with casters versus walking with the powered base

\begin{tabular}{|c|c|c|}
\hline & $\operatorname{RMS} f_{y, i}(\mathrm{~N})$ & Mean Push-Off Force $(\mathrm{N})$ \\
\hline With casters & $48.7(2.7)$ & $112.5(18.9)$ \\
With powered mobile base & $15.4(1.2)$ & $36.3(11.9)$ \\
\hline
\end{tabular}

\subsection{Investigating the Effects of the Passive Linkage}

As noted in Section 5.2, the walking experiments were performed with the user-device interface that bypassed GaitEnable's passive linkage. This ensured that the dynamics of the passive linkage did not influence the results. In order to investigate the effects of the passive linkage, one participant was requested to repeat the walking experiments with the user-device interface attached to the passive linkage as shown in Figure 4.9. All remaining controller parameters were specified as in test condition 4 of the walking experiments.

The user performed three data collection test runs with the user-device interface attached to the passive linkage. Interaction force data and mobile base kinematics were recorded during all three test runs. Table 5.6 presents the RMS force and velocity data and the mean vales of $W$ from both test conditions. The table shows that the RMS values of $\dot{y}$ were similar at both test conditions. This suggests that there was no major difference in the participant's walking pattern. However, the mean force inputs required to move GaitEnable, and the effective mass that GaitEnable displayed in the forward direction were reduced by $10 \%$. This means that the passive linkage enabled the participant to move GaitEnable with smaller force inputs, likely because the free motion of the passive linkage allowed him to accelerate his pelvis more easily when initiating a step.

Interestingly, the participant suggested that there was no perceptible difference between walking with and without the passive linkage. This is likely because the effect of the assistance force dominated the interaction. Considering that the mean value of $W$ calculated for the participant at the zero assistance force test condition was $24.6 \mathrm{~J}$ (i.e., at test condition 2), the addition of the $20 \mathrm{~N}$ assistance force changed $W$ by 27 J. However, walking with 


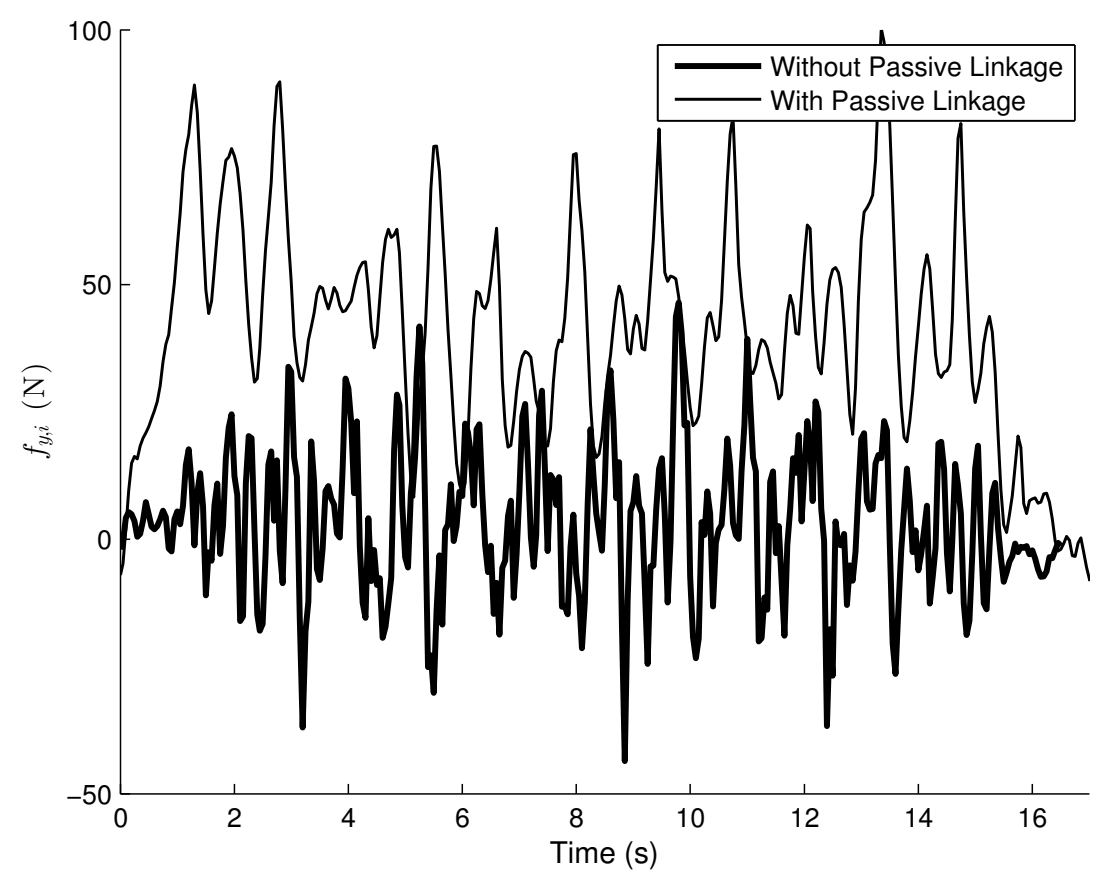

Figure 5.8: Comparing the fore/aft interaction force with casters versus the powered mobile base. With the powered based, the push-off force required to initiate motion and the mean force required to propel the mobile base are significantly reduced.

and without a passive linkage only caused a change in $W$ of $7 \mathrm{~J}$. Thus, it is probable that the sensation of the assistance force is more dramatic than the sensation of the passive linkage. Though more experiments are necessary to confirm this, this preliminary result suggests that future prototypes of GaitEnable could be designed without a passive linkage altogether if the mobile base's responsiveness can be improved with more powerful actuators.

Table 5.6: Comparing walking with and without the passive linkage

\begin{tabular}{|c|c|c|c|c|}
\hline & $\begin{array}{c}f_{y, i}(\mathrm{~N}) \\
\text { mean (s.d.) }\end{array}$ & $\begin{array}{c}\hat{m}_{y}(\mathrm{Kg}) \\
\text { mean (s.d.) }\end{array}$ & $\begin{array}{c}\dot{y}(\mathrm{~m} / \mathrm{s}) \\
\text { mean (s.d.) }\end{array}$ & $\begin{array}{c}W(\mathrm{~J}) \\
\text { mean (s.d.) }\end{array}$ \\
\hline With passive linkage & $13.6(0.2)$ & $27.3(0.3)$ & $0.26(0.002)$ & $-2.4(0.6)$ \\
Without passive linkage & $15.4(1.2)$ & $31.4(1.6)$ & $0.26(0.007)$ & $-9.3(1.0)$ \\
\hline
\end{tabular}




\subsection{Chapter Summary}

This chapter described a series of experiments carried out to validate the performance of GaitEnable's mobile base and interaction control system. The results confirm that healthy subjects can use the pelvis-based interaction control system to command the motion of the device, and that the impedance manipulation ensure stable-user device interactions. Though omniwheel vibrations, the use of the impedance manipulations, the limitations of the servomotors, and the inertial loads of the pelvis constraint structure all interfere with the impedance display, GaitEnable can still display a peak $70 \%$ mass reduction when the effect of assistance forces are factored in. Moreover, changing the desired mass parameter results in a predictable change in the behaviour of the device, even though GaitEnable is incapable of accurately displaying the desired impedance.

Other experiments demonstrate that 10 and $20 \mathrm{~N}$ virtual assistance forces applied through the interaction model reduce the force inputs required to move GaitEnable by 20\%. GaitEnable's omnidirectional mobile base is also shown to reduce lateral interaction forces by over $220 \%$ in comparison to the scenario of walking with constrained lateral pelvis translations. GaitEnable's ability to generate change-in-support reactions was also tested, and the results confirm that GaitEnable is effective at invoking change-in-support reactions due to lateral disturbances. A final set of experiments also demonstrate that the mobile base and interaction control system reduce the mean propulsion force required to sustain GaitEnable's motion by $300 \%$, and that the effect of a virtual assistance force is more perceptible than the effect of the passive linkage.

Overall, the results confirm that the interaction control system can synchronize the device's motion with the user's voluntary motion, and that it can generate force cues for assisting or perturbing the user's gait. However, additional studies are required to confirm GaitEnable's ability to emulate normal human gait, and to determine if the mobile base's ability to assist walking is sufficient for the frail patients who are most likely to use the 
device. Such experiments are currently being planned to be executed in collaboration with local hospitals. 


\section{Chapter 6}

\section{Conclusion and Recommendations}

Robots for gait rehabilitation can enhance patient safety, reduce therapist workload, and enable new training possibilities. However, most devices available today are large, complicated, and expensive, and offer limited training capabilities. Accordingly, this research investigated the conceptual design, development, and experimental validation of a robotic gait trainer that facilitates overground gait and balance training. GaitEnable, the device developed to support the research, combines an actuated omnidirectional mobile base, an interaction control system, and a passive body weight support system. Both its omniwheel mobile base and its pelvis-driven interaction control system make it unique with respect to other gait trainers.

GaitEnable's interaction control system consists of an admittance controller that transforms the interaction forces measured at the user-device interface into motion commands for the device. The need to ensure stable user-device interactions, particularly when GaitEnable displayed a small mass, motivated the development of an enhanced admittance controller. In particular, two impedance manipulations that reshape and limit a haptic device's commanded acceleration were designed, analyzed and experimentally validated. Reshaping the commanded acceleration with error feedback limits the divergence of tracking error, and attenuates sustained oscillations in a haptic device's response. The dynamic, error-dependent limit on the reference acceleration ensures bounded position tracking errors. A novel dual-loop position controller, stabilized by 
these impedance manipulations, was also proposed, analyzed, and experimentally validated.

The enhanced admittance controller, derived for both joint-space and task-space admittance control, results in simple-to-implement, model-free admittance control with guaranteed stability, and the unique ability to safely display small apparent inertias. Experiments performed with a 1-DOF haptic device demonstrated that the enhanced admittance controller allowed stable interactions under a wide range of controller gains, actuator saturation, and at apparent inertia reductions as large as 99.3\%. Experiments performed with 10 healthy subjects also confirmed that subject-specific effects cannot destabilize the controller. Additionally, experiments for validating the dual-loop position controller demonstrated that the impedance manipulations allow the use of higher position control gains and improved tracking performance, and that error feedback in the reference model is effective for attenuating chatter observed with conventional PD controllers.

The impedance manipulations were adapted for use with GaitEnable, and 7 healthy subjects were recruited to perform a series of experiments to validate the performance of the system. The experimental results confirmed that users could command the motion of GaitEnable by modulating their pelvis motion, and that the impedance manipulations ensured stable user-device interactions. Changing GaitEnable's desired mass from $20 \mathrm{~kg}$ to $4 \mathrm{~kg}$ generated a noticeable reduction in the effort required to move the device, and virtual assistance forces applied through the admittance model were shown to reduce the mean force inputs required to move the device. A comparison of walking with and without constrained lateral pelvis translations demonstrated that omnidirectional base motions minimized the interaction forces generated at the user-device interface. Additional experiments demonstrated that GaitEnable can generate disturbances for invoking change-in-support reactions, and that the actuated mobile base reduces the peak push-off and mean propulsion forces required to propel GaitEnable by 300\%. These results confirm that GaitEnable can influence a user's gait in a consistent and predictable manner, even though omniwheel vibrations and the impedance manipulations reduce the fidelity of the 
impedance display. The results also confirm that GaitEnable addresses several limitations of other passive and robotic gait trainers, as it can display a small mass, reduce motion constraints, and actively assist or perturb a user.

\subsection{Future Work and Recommendations}

The analysis and experimental validation of the impedance manipulations presented in this thesis has laid a foundation for future studies that explore similar model-free admittance and position control structures. The following list presents suggestions for areas that require further study:

- The impedance manipulations require accurate position, velocity and acceleration feedback. As such, additional studies that analyze how sensor noise and filtering effects influence their performance and stability would be beneficial.

- The acceleration limits were derived with the intention of bounding the Lyapunov function for the error dynamics from above and below. If a different formulation of the error dynamics is used, or if a different candidate Lyapunov function is selected, then analogous acceleration limits could be defined for imposing the UUB stability condition. Such analyses may yield more favourable expressions of the impedance manipulations that do not require velocity or acceleration feedback. It would also be interesting to explore whether this concept can be extended for stabilizing observer error dynamics and parameter estimation error dynamics in adaptive control.

- Theorems 1 and 2 in Chapter 3 show that the bound on the position tracking error guaranteed by the acceleration limits is a ratio of several parameters. Remark 1 of Theorem 1 provides some guidelines for how these parameters can be selected. However, many different controller parameter combinations will yield the same tracking error bound. Thus, additional analysis and experiments could be performed to identify which parameter combinations yield the best overall controller performance. 
The experimental evaluation of GaitEnable confirmed that healthy subjects can use the pelvis-driven interaction control to command the device, and that changes to the interaction model predictably affect a user's motion. As the second prototype of GaitEnable has already addressed many of the outstanding mechanical and useability issues, the list below focuses on suggestions for additional experiments and improvements required to prepare the device for patient use:

- Experiments with a wide variety of patient populations would be beneficial for understanding which populations are best suited to use the device.

- Additional research can also be undertaken to better link the design of the interaction model to therapy requirements. Such research may result in the development of software modules for specific impairments, or modified control systems that use information about the user's impairment severity, balance, and exertion level.

- Displaying a small mass, and applying pushing forces to make the device easy to move may not be adequate for some patients, or optimal from a therapeutic perspective. Thus, different concepts of assistance could designed and tested with patients. This may require fusing information from different modalities such as EMG and EEG, or implementing intelligent assistance algorithms that adapt assistance according a user's exertion level.

- The design of the platform should be modified to comply with a variety of safety standards such as the IEC 60601, ISO 10535, and ISO 13482 standards. Compliance to these standards is required by regulatory bodies such as Health Canada and the Food and Drug Administration in the United States of America before large-scale clinical trials in hospitals can be undertaken. 


\section{List of References}

[1] Heart and S. F. Canada., "Stroke is urgent: Low awareness puts women at risk of cutting their lives short." http://www.heartandstroke.on.ca.

[2] J. Patton, D. A. Brown, M. Peshkin, J. J. Santos-Munn, A. Makhlin, E. Lewis, E. J. Colgate, and D. Schwandt, "Kineassist: design and development of a robotic overground gait and balance therapy device.," Top Stroke Rehabil, vol. 15, no. 2, pp. 131-139, 2008.

[3] R. K. Bode, A. W. Heinemann, P. Semik, and T. Mallinson, "Relative importance of rehabilitation therapy characteristics on functional outcomes for persons with stroke.," Stroke, vol. 35, pp. 2537-2542, Nov 2004.

[4] K. J. Sullivan, D. A. Brown, T. Klassen, S. Mulroy, T. Ge, S. P. Azen, C. J. Winstein, and P. T. C. R. N. (PTClinResNet), "Effects of task-specific locomotor and strength training in adults who were ambulatory after stroke: results of the steps randomized clinical trial.," Phys Ther, vol. 87, pp. 1580-1602, Dec 2007.

[5] M. C. Creditor, "Hazards of hospitalization of the elderly.," Ann Intern Med, vol. 118, pp. 219-223, Feb 1993.

[6] A. L. Siu, J. D. Penrod, K. S. Boockvar, K. Koval, E. Strauss, and R. S. Morrison, "Early ambulation after hip fracture: effects on function and mortality.," Arch Intern Med, vol. 166, pp. 766-771, Apr 2006.

[7] S. R. Fisher, Y. fang Kuo, J. E. Graham, K. J. Ottenbacher, and G. V. Ostir, "Early ambulation and length of stay in older adults hospitalized for acute illness.," Arch Intern Med, vol. 170, pp. 1942-1943, Nov 2010.

[8] P. E. Morris, A. Goad, C. Thompson, K. Taylor, B. Harry, L. Passmore, A. Ross, L. Anderson, S. Baker, M. Sanchez, L. Penley, A. Howard, L. Dixon, S. Leach, R. Small, R. D. Hite, and E. Haponik, "Early intensive care unit mobility therapy in the treatment of acute respiratory failure.," Crit Care Med, vol. 36, pp. 2238-2243, Aug 2008. 
[9] D. M. Needham, R. Korupolu, J. M. Zanni, P. Pradhan, E. Colantuoni, J. B. Palmer, R. G. Brower, and E. Fan, "Early physical medicine and rehabilitation for patients with acute respiratory failure: a quality improvement project.," Arch Phys Med Rehabil, vol. 91, pp. 536-542, Apr 2010.

[10] L. B. Oldmeadow, E. R. Edwards, L. A. Kimmel, E. Kipen, V. J. Robertson, and M. J. Bailey, "No rest for the wounded: early ambulation after hip surgery accelerates recovery.," ANZ J Surg, vol. 76, pp. 607-611, Jul 2006.

[11] "National hospital discharge survey: 2007 summary," tech. rep., Centers for Disease Control and Prevention, 2010.

[12] D. Condra, "An assessment of falls in patients rooms and methods of prevention," tech. rep., 2010.

[13] L. U.S. Senate Committee on Health, Education, P. S. on Employment, and W. Safety, "Testimony of james w. collins. subcommittee hearing - safe patient handling \& lifting standards for a safer american workforc," tech. rep., 2010.

[14] S. Hesse and D. Uhlenbrock, "A mechanized gait trainer for restoration of gait.," $J$ Rehabil Res Dev, vol. 37, no. 6, pp. 701-708, 2000.

[15] H. Schmidt, S. Hesse, R. Bernhardt, and J. Krüger, "Hapticwalker - a novel haptic foot device," ACM Trans. Appl. Percept., vol. 2, no. 2, pp. 166-180, 2005.

[16] S. Freivogel, J. Mehrholz, T. Husak-Sotomayor, and D. Schmalohr, "Gait training with the newly developed 'lokohelp'-system is feasible for non-ambulatory patients after stroke, spinal cord and brain injury. a feasibility study.," Brain Inj, vol. 22, pp. 625-632, Jul 2008.

[17] S. Hesse, A. Waldner, and C. Tomelleri, "Innovative gait robot for the repetitive practice of floor walking and stair climbing up and down in stroke patients.," J Neuroeng Rehabil, vol. 7, p. 30, 2010.

[18] J. Yoon, B. Novandy, C.-H. Yoon, and K.-J. Park, "A 6-dof gait rehabilitation robot with upper and lower limb connections that allows walking velocity updates on various terrains," Mechatronics, IEEE/ASME Transactions on, vol. 15, pp. 201 -215, apr. 2010.

[19] G. Colombo, M. Wirz, and V. Dietz, "Driven gait orthosis for improvement of locomotor training in paraplegic patients.," Spinal Cord, vol. 39, pp. 252-255, May 2001.

[20] D. Aoyagi, W. E. Ichinose, S. J. Harkema, D. J. Reinkensmeyer, and J. E. Bobrow, "A robot and control algorithm that can synchronously assist in naturalistic motion 
during body-weight-supported gait training following neurologic injury.," IEEE Trans Neural Syst Rehabil Eng, vol. 15, pp. 387-400, Sep 2007.

[21] J. F. Veneman, R. Kruidhof, E. E. G. Hekman, R. Ekkelenkamp, E. H. F. V. Asseldonk, and H. van der Kooij, "Design and evaluation of the lopes exoskeleton robot for interactive gait rehabilitation.," IEEE Trans Neural Syst Rehabil Eng, vol. 15, pp. 379-386, Sep 2007.

[22] P. Beyl, M. Van Damme, R. Van Ham, B. Vanderborght, and D. Lefeber, "Design and control of a lower limb exoskeleton for robot-assisted gait training," Applied Bionics and Biomechanics, vol. 6, no. 2, pp. 229-243, 2009.

[23] S. K. Banala, S. H. Kim, S. K. Agrawal, and J. P. Scholz, "Robot assisted gait training with active leg exoskeleton (alex).," IEEE Trans Neural Syst Rehabil Eng, vol. 17, pp. 2-8, Feb 2009.

[24] J. A. Kleim and T. A. Jones, "Principles of experience-dependent neural plasticity: implications for rehabilitation after brain damage.," J Speech Lang Hear Res, vol. 51, pp. S225-S239, Feb 2008.

[25] L. Finch and H. Barbeau, "Influence of partial weight bearing on normal human gait: the development of a gait retraining strategy," Can. J. Neurolog. Sci., vol. 12, p. 183, 1985 .

[26] V. Dietz, G. Colombo, and L. Jensen, "Locomotor activity in spinal man.," Lancet, vol. 344, pp. 1260-1263, Nov 1994.

[27] S. Hesse, M. Malezic, A. Schaffrin, and K. H. Mauritz, "Restoration of gait by combined treadmill training and multichannel electrical stimulation in non-ambulatory hemiparetic patients.," Scand J Rehabil Med, vol. 27, pp. 199-204, Dec 1995.

[28] M. Visintin, H. Barbeau, N. Korner-Bitensky, and N. E. Mayo, "A new approach to retrain gait in stroke patients through body weight support and treadmill stimulation.," Stroke, vol. 29, pp. 1122-1128, Jun 1998.

[29] S. Hesse, M. Konrad, and D. Uhlenbrock, "Treadmill walking with partial body weight support versus floor walking in hemiparetic subjects.," Arch Phys Med Rehabil, vol. 80, pp. 421-427, Apr 1999.

[30] A. Wernig, A. Nanassy, and S. Mller, "Laufband (treadmill) therapy in incomplete paraplegia and tetraplegia.," J Neurotrauma, vol. 16, pp. 719-726, Aug 1999.

[31] V. R. Edgerton, R. D. Leon, S. J. Harkema, J. A. Hodgson, N. London, D. J. Reinkensmeyer, R. R. Roy, R. J. Talmadge, N. J. Tillakaratne, W. Timoszyk, and A. Tobin, "Retraining the injured spinal cord.," J Physiol, vol. 533, pp. 15-22, May 2001. 
[32] A. L. Behrman and S. J. Harkema, "Locomotor training after human spinal cord injury: a series of case studies.," Phys Ther, vol. 80, pp. 688-700, Jul 2000.

[33] H. L. R. C. (UCLA), "Locomotor training." http://www.harkema.ucla.edu/LTpp.html.

[34] H. Barbeau and M. Visintin, "Optimal outcomes obtained with body-weight support combined with treadmill training in stroke subjects.," Arch Phys Med Rehabil, vol. 84, pp. 1458-1465, Oct 2003.

[35] M. Frey, G. Colombo, M. Vaglio, R. Bucher, M. Jrg, and R. Riener, "A novel mechatronic body weight support system.," IEEE Trans Neural Syst Rehabil Eng, vol. 14, pp. 311-321, Sep 2006.

[36] J. Liang, A. Hernandez, O. Ma, B. Qiao, and R. Paz, "Nonhuman test of a new active body support system for improving locomotion training," pp. $1312-1317$, jul. 2009.

[37] M. Glauser, Z. Lin, and P. Allaire, "Modeling and control of a partial body weight support system: An output regulation approach," Control Systems Technology, IEEE Transactions on, vol. 18, pp. $480-490$, mar. 2010.

[38] K. E. Gordon, D. P. Ferris, M. Robertson, J. A. Beres, and S. J. Harkema, "The importance of using an appropriate body weight support system in locomotor training," in Soc. Neurosci., p. Abstract 26 (PN 61.9), 2000.

[39] F. Gazzani, A. Fadda, M. Torre, and V. Macellari, "Ward: a pneumatic system for body weight relief in gait rehabilitation.," IEEE Trans Rehabil Eng, vol. 8, pp. 506513 , Dec 2000.

[40] T. Watanabe, E. Ohki, T. Ando, and M. Fujie, "Fundamental study of force control method for pelvis-supporting body weight support system," pp. 1403 -1408, feb. 2009.

[41] G. Chen, D. Schwandt, H. F. M. V. der Loos, J. Anderson, D. P. Ferris, F. E. Zajac, S. A. Kautz, C. G. Burgar, C. Patten, R. R. Neptune, and K. E. Gordon, "Complianceadjustable, force-sensing harness support for studying treadmill training in neurologically impaired subjects," Presented at the 6th Annu. Gait Clin. Movement Anal. Meeting, Sacramento, CA, 2001.

[42] J. R. Franz, M. Glauser, P. O. Riley, U. D. Croce, F. Newton, P. E. Allaire, and D. C. Kerrigan, "Physiological modulation of gait variables by an active partial body weight support system.," J Biomech, vol. 40, no. 14, pp. 3244-3250, 2007.

[43] S. J. Lee and J. Hidler, "Biomechanics of overground vs. treadmill walking in healthy individuals.," J Appl Physiol, vol. 104, pp. 747-755, Mar 2008.

[44] J. Collett, H. Dawes, K. Howells, C. Elsworth, H. Izadi, and C. Sackley, "Anomalous centre of mass energy fluctuations during treadmill walking in healthy individuals.," Gait Posture, vol. 26, pp. 400-406, Sep 2007. 
[45] M. Pohl, J. Mehrholz, C. Ritschel, and S. Rckriem, "Speed-dependent treadmill training in ambulatory hemiparetic stroke patients: a randomized controlled trial.," Stroke, vol. 33, pp. 553-558, Feb 2002.

[46] L. Ada, C. M. Dean, M. E. Morris, J. M. Simpson, and P. Katrak, "Randomized trial of treadmill walking with body weight support to establish walking in subacute stroke: the mobilise trial.," Stroke, vol. 41, pp. 1237-1242, Jun 2010.

[47] J. F. Veneman, J. Menger, E. H. F. van Asseldonk, F. C. T. van der Helm, and H. van der Kooij, "Fixating the pelvis in the horizontal plane affects gait characteristics.," Gait Posture, vol. 28, pp. 157-163, Jul 2008.

[48] J. M. Donelan, D. W. Shipman, R. Kram, and A. D. Kuo, "Mechanical and metabolic requirements for active lateral stabilization in human walking.," $J$ Biomech, vol. 37, pp. 827-835, Jun 2004.

[49] D. Saha, S. Gard, and S. Fatone, "The effect of trunk flexion on able-bodied gait.," Gait Posture, vol. 27, pp. 653-660, May 2008.

[50] M. K. Aaslund and R. Moe-Nilssen, "Treadmill walking with body weight support effect of treadmill, harness and body weight support systems.," Gait Posture, vol. 28, pp. 303-308, Aug 2008.

[51] J. M. Hidler and A. E. Wall, "Alterations in muscle activation patterns during roboticassisted walking.," Clin Biomech (Bristol, Avon), vol. 20, pp. 184-193, Feb 2005.

[52] M. Woollacott and A. Shumway-Cook, "Attention and the control of posture and gait: a review of an emerging area of research.," Gait Posture, vol. 16, pp. 1-14, Aug 2002.

[53] B. J. Vellas, S. J. Wayne, L. Romero, R. N. Baumgartner, L. Z. Rubenstein, and P. J. Garry, "One-leg balance is an important predictor of injurious falls in older persons.," J Am Geriatr Soc, vol. 45, pp. 735-738, Jun 1997.

[54] M. E. Tinetti and C. Kumar, "The patient who falls: "it's always a trade-off".," $J A M A$, vol. 303, pp. 258-266, Jan 2010.

[55] D. S. Marigold, J. J. Eng, A. S. Dawson, J. T. Inglis, J. E. Harris, and S. Gylfadttir, "Exercise leads to faster postural reflexes, improved balance and mobility, and fewer falls in older persons with chronic stroke.," J Am Geriatr Soc, vol. 53, pp. 416-423, Mar 2005.

[56] S. W. Muir, K. Berg, B. Chesworth, N. Klar, and M. Speechley, "Quantifying the magnitude of risk for balance impairment on falls in community-dwelling older adults: a systematic review and meta-analysis.," J Clin Epidemiol, vol. 63, pp. 389-406, Apr 2010. 
[57] A. Mansfield, A. L. Peters, B. A. Liu, and B. E. Maki, "Effect of a perturbation-based balance training program on compensatory stepping and grasping reactions in older adults: a randomized controlled trial.," Phys Ther, vol. 90, pp. 476-491, Apr 2010.

[58] F. M. Alfieri, R. R. de Jesus Guirro, and R. M. Teodori, "Postural stability of elderly submmitted to multisensorial physical therapy intervention.," Electromyogr Clin Neurophysiol, vol. 50, pp. 113-119, Mar 2010.

[59] E. D. de Bruin and K. Murer, "Effect of additional functional exercises on balance in elderly people.," Clin Rehabil, vol. 21, pp. 112-121, Feb 2007.

[60] D. Rugelj, "The effect of functional balance training in frail nursing home residents.," Arch Gerontol Geriatr, vol. 50, no. 2, pp. 192-197, 2010.

[61] A. Hammer, Y. Nilsagard, and M. Wallquist, "Balance training in stroke patients - a systematic review of randomized, controlled trials," Advances in Physiotherapy, vol. 10, no. 4, pp. 163-172, 2008.

[62] N. Goljar, H. Burger, M. Rudolf, and I. Stanonik, "Improving balance in subacute stroke patients: a randomized controlled study.," Int J Rehabil Res, vol. 33, pp. 205210, Sep 2010.

[63] A. Lubetzky-Vilnai and D. Kartin, "The effect of balance training on balance performance in individuals poststroke: a systematic review.," J Neurol Phys Ther, vol. 34, pp. 127-137, Sep 2010.

[64] I. G. L. van de Port, S. Wood-Dauphinee, E. Lindeman, and G. Kwakkel, "Effects of exercise training programs on walking competency after stroke: a systematic review.," Am J Phys Med Rehabil, vol. 86, pp. 935-951, Nov 2007.

[65] R. Orr, J. Raymond, and M. F. Singh, "Efficacy of progressive resistance training on balance performance in older adults : a systematic review of randomized controlled trials.," Sports Med, vol. 38, no. 4, pp. 317-343, 2008.

[66] B. E. Maki, K. C.-C. Cheng, A. Mansfield, C. Y. Scovil, S. D. Perry, A. L. Peters, S. McKay, T. Lee, A. Marquis, P. Corbeil, G. R. Fernie, B. Liu, and W. E. McIlroy, "Preventing falls in older adults: new interventions to promote more effective changein-support balance reactions.," J Electromyogr Kinesiol, vol. 18, pp. 243-254, Apr 2008.

[67] D. Hyndman, A. Ashburn, and E. Stack, "Fall events among people with stroke living in the community: circumstances of falls and characteristics of fallers.," Arch Phys Med Rehabil, vol. 83, pp. 165-170, Feb 2002. 
[68] E. Stack and A. Ashburn, "Fall events described by people with parkinson's disease: implications for clinical interviewing and the research agenda.," Physiother Res Int, vol. 4, no. 3, pp. 190-200, 1999.

[69] R. G. Cumming and R. J. Klineberg, "Fall frequency and characteristics and the risk of hip fractures.," J Am Geriatr Soc, vol. 42, pp. 774-778, Jul 1994.

[70] S. H. Hystem, "Lokomat robotic gait rehabilitation." http://www.shorehealth.org/services/rehabilitation/lokomat.shtml, 2010.

[71] M. Peshkin, D. Brown, J. Santos-Munne, A. Makhlin, E. Lewis, J. Colgate, J. Patton, and D. Schwandt, "Kineassist: a robotic overground gait and balance training device," pp. $241-246$, jun. 2005.

[72] K.-H. Seo and J.-J. Lee, "The development of two mobile gait rehabilitation systems.," IEEE Trans Neural Syst Rehabil Eng, vol. 17, pp. 156-166, Apr 2009.

[73] Y. Stauffer, Y. Allemand, M. Bouri, J. Fournier, R. Clavel, P. Metrailler, R. Brodard, and F. Reynard, "The walktrainer; a new generation of walking reeducation device combining orthoses and muscle stimulation," Neural Systems and Rehabilitation Engineering, IEEE Transactions on, vol. 17, pp. 38 -45, feb. 2009.

[74] J. Mehrholz, C. Werner, J. Kugler, and M. Pohl, "Electromechanical-assisted gait training with physiotherapy may improve walking after stroke.," Stroke, Apr 2008.

[75] S. Hussein, H. Schmidt, M. Volkmar, C. Werner, I. Helmich, F. Piorko, J. Krger, and S. Hesse, "Muscle coordination in healthy subjects during floor walking and stair climbing in robot assisted gait training.," Conf Proc IEEE Eng Med Biol Soc, vol. 2008, pp. 1961-1964, 2008.

[76] M. Ng, R. Tong, and L. Li, "A pilot study of randomized clinical controlled trial of gait training in subacute stroke patients with partial body-weight support electromechanical gait trainer and functional electrical stimulation: six-month follow-up.," Stroke, vol. 39, pp. 154-160, Jan 2008.

[77] H. van der Kooij, J. Veneman, and R. Ekkelenkamp, "Design of a compliantly actuated exo-skeleton for an impedance controlled gait trainer robot.," Conf Proc IEEE Eng Med Biol Soc, vol. 1, pp. 189-193, 2006.

[78] R. Riener, L. Lnenburger, S. Jezernik, M. Anderschitz, G. Colombo, and V. Dietz, "Patient-cooperative strategies for robot-aided treadmill training: first experimental results.," IEEE Trans Neural Syst Rehabil Eng, vol. 13, pp. 380-394, Sep 2005.

[79] J.-P. Regnaux, K. Saremi, J. Marehbian, B. Bussel, and B. H. Dobkin, "An accelerometry-based comparison of 2 robotic assistive devices for treadmill training of gait.," Neurorehabil Neural Repair, vol. 22, no. 4, pp. 348-354, 2008. 
[80] K. P. Westlake and C. Patten, "Pilot study of lokomat versus manual-assisted treadmill training for locomotor recovery post-stroke.," J Neuroeng Rehabil, vol. 6, p. 18, 2009.

[81] M. Schwaiger, T. Thummel, and H. Ulbrich, "Cyberwalk: An advanced prototype of a belt array platform," pp. $50-55$, oct. 2007.

[82] C.-Y. Lee and J.-J. Lee, "Walking-support robot system for walking rehabilitation: design and control," Artificial Life and Robotics, vol. 4, pp. 206-211, 2000. 10.1007/BF02481176.

[83] L. Saint-Bauzel, V. Pasqui, and I. Monteil, "A reactive robotized interface for lower limb rehabilitation: Clinical results," Robotics, IEEE Transactions on, vol. 25, pp. 583 -592 , jun. 2009 .

[84] B. Graf, M. Hans, and R. Schraft, "Care-o-bot ii - development of a next generation robotic home assistant," Autonomous Robots, vol. 16, pp. 193-205, 2004. 10.1023/B:AURO.0000016865.35796.e9.

[85] J. Yang, W. Zhao, D. Bai, and S. Wang, "Omnidirectional lower limbs rehabilitation training robot based on biofeedback," in ICIT '09: Proceedings of the 2009 IEEE International Conference on Industrial Technology, (Washington, DC, USA), pp. 1-5, IEEE Computer Society, 2009.

[86] J. Glover, D. Holstius, M. Manojlovich, K. Montgomery, A. Powers, J. Wu, S. Kiesler, J. Matthews, and S. Thrun, "A robotically-augmented walker for older adults," Tech. Rep. CMU-CS-03-170, Carnegie Mellon University, Computer Science Department, Pittsburgh, PA, 2003.

[87] S. Dubowsky, F. Genot, S. Godding, H. Kozono, A. Skwersky, H. Yu, and L. S. $\mathrm{Yu}$, "Pamm - a robotic aid to the elderly for mobility assistance and monitoring: a ldquo;helping-hand rdquo; for the elderly," vol. 1, pp. 570 -576 vol.1, 2000.

[88] G. Lacey and D. Rodriguez-Losada, "The evolution of guido," Robotics Automation Magazine, IEEE, vol. 15, pp. 75 -83, dec. 2008.

[89] H. Kazerooni and R. Steger, "That which does not stabilize, will only make us stronger," in Robotics Research (S. Thrun, R. Brooks, and H. Durrant-Whyte, eds.), vol. 28 of Springer Tracts in Advanced Robotics, pp. 373-395, Springer Berlin / Heidelberg, 2007.

[90] C. Technologies, "Robot suit hal." http://www.cyberdyne.jp/english/robotsuithal/index.html.

[91] A. M. Technologies, "The rewalk robotic suit." http://www.argomedtec.com/products.asp. 
[92] J. E. Pratt, B. T. Krupp, C. J. Morse, and S. H. Collins, "The roboknee: an exoskeleton for enhancing strength and endurance during walking," in Proc. IEEE International Conference on Robotics and Automation ICRA '04, vol. 3, pp. 2430-2435, Apr 26-May 1, 2004.

[93] B. Bionics. http://www.berkeleybionics.com/.

[94] G. Mone, "Building the real iron man." http://www.popsci.com/node/20689.

[95] N. T. Inc., "The bungee mobility trainer." http://www.neurogymtech.com/.

[96] H. van der Kooij, B. Koopman, and E. H. F. van Asseldonk, "Body weight support by virtual model control of an impedance controlled exoskeleton (lopes) for gait training.," Conf Proc IEEE Eng Med Biol Soc, vol. 2008, pp. 1969-1972, 2008.

[97] W. Zijlstra and A. L. Hof, "Displacement of the pelvis during human walking: experimental data and model predictions," Gait $\mathscr{E}$ Posture, vol. 6, no. 3, pp. 249 - 262, 1997.

[98] L. Zhao, L. Zhang, L. Wang, and J. Wang, "Three-dimensional motion of the pelvis during human walking," vol. 1, pp. 335 - 339 Vol. 1, jul. 2005.

[99] E. T. Wolbrecht, V. Chan, D. J. Reinkensmeyer, and J. E. Bobrow, "Optimizing compliant, model-based robotic assistance to promote neurorehabilitation," IEEE Transactions on Neural Systems and Rehabilitation Engineering, vol. 16, pp. 286-297, June 2008.

[100] N. Hogan, H. I. Krebs, B. Rohrer, J. J. Palazzolo, L. Dipietro, S. E. Fasoli, J. Stein, R. Hughes, W. R. Frontera, D. Lynch, and B. T. Volpe, "Motions or muscles? Some behavioral factors underlying robotic assistance of motor recovery," Journal of Rehabilitation Research \&s Development, vol. 43, pp. 605-618, Aug. 2006.

[101] C. Fleischer and G. Hommel, "Emg-driven human model for orthosis control," Human Interaction with Machines, pp. 69-76, 2006.

[102] D. Novak, X. Omlin, R. Leins-Hess, and R. Riener, "Predicting targets of human reaching motions using different sensing technologies," Biomedical Engineering, IEEE Transactions on, vol. 60, no. 9, pp. 2645-2654, 2013.

[103] L. Marchal-Crespo and D. J. Reinkensmeyer, "Review of control strategies for robotic movement training after neurologic injury.," J Neuroeng Rehabil, vol. 6, p. 20, 2009.

[104] S. Hara, "A smooth switching from power-assist control to automatic transfer control and its application to a transfer machine," Industrial Electronics, IEEE Transactions on, vol. 54, pp. 638-650, feb. 2007. 
[105] B. Stanczyk and M. Buss, "Experimental comparison of interaction control methods for a redundant telemanipulator," in in Proceedings of the International Symposium on Methods and Models in Automation and Robotics MMAR2005, pp. 677-682, 2005.

[106] R. Riener, M. Frey, T. Proll, F. Regenfelder, and R. Burgkart, "Phantom-based multimodal interactions for medical education and training: the munich knee joint simulator," Information Technology in Biomedicine, IEEE Transactions on, vol. 8, pp. 208216 , june 2004 .

[107] A. M. Okamura, "Methods for haptic feedback in teleoperated robot-assisted surgery.," Ind Rob, vol. 31, pp. 499-508, Dec 2004.

[108] C. Ott, R. Mukherjee, and Y. Nakamura, "Unified impedance and admittance control," in Robotics and Automation (ICRA), 2010 IEEE International Conference on, pp. 554-561, may 2010.

[109] D. Lawrence, "Impedance control stability properties in common implementations," pp. $1185-1190$ vol.2, Apr. 1988.

[110] A. Peer and M. Buss, "Robust stability analysis of a bilateral teleoperation system using the parameter space approach," in Intelligent Robots and Systems, 2008. IROS 2008. IEEE/RSJ International Conference on, pp. 2350 -2356, sept. 2008.

[111] M. Ueberle and M. Buss, "Control of kinesthetic haptic interfaces," in Proc. IEEE/RSJ Int. Conf. Intell. Robots Syst., Workshop Touch Haptics,, pp. 147-151, 2004.

[112] G. Magallan, C. De Angelo, and G. Garcia, "Maximization of the traction forces in a 2wd electric vehicle," Vehicular Technology, IEEE Transactions on, vol. 60, pp. 369 -380 , feb. 2011.

[113] V. Colli, G. Tomassi, and M. Scarano, "single wheel" longitudinal traction control for electric vehicles," Power Electronics, IEEE Transactions on, vol. 21, pp. 799 -808, may 2006.

[114] M. Amodeo, A. Ferrara, R. Terzaghi, and C. Vecchio, "Wheel slip control via secondorder sliding-mode generation," Intelligent Transportation Systems, IEEE Transactions on, vol. 11, pp. $122-131$, march 2010.

[115] A. Peer, Design and Control of Admittance-Type Telemanipulation Systems. PhD thesis, Technical University Munich, 2008.

[116] N. Colonnese and A. Okamura, "M-width: Stability and accuracy of haptic rendering of virtual mass," in Robotics: Science and Systems, (Sydney, Australia), July 9-13 2012. 
[117] A. Haddadi and K. Hashtrudi-Zaad, "A new robust stability analysis and design tool for bilateral teleoperation control systems," in Robotics and Automation, 2008. ICRA 2008. IEEE International Conference on, pp. 663 -670, may 2008.

[118] R. Adams and B. Hannaford, "Control law design for haptic interfaces to virtual reality," Control Systems Technology, IEEE Transactions on, vol. 10, pp. 3 -13, jan 2002.

[119] S. Buerger and N. Hogan, "Complementary stability and loop shaping for improved human and robot interaction," Robotics, IEEE Transactions on, vol. 23, pp. $232-244$, april 2007.

[120] D. Surdilovic, "Robust control design of impedance control for industrial robots," in Intelligent Robots and Systems, 200\%. IROS 200\%. IEEE/RSJ International Conference on, pp. 3572 -3579, 29 2007-nov. 22007.

[121] M. T. Ahmadian, G. Vossoughi, and F. Tajaddodianfar, "Robust stable control of haptic devices based on transparency maximization," Proceedings of the Institution of Mechanical Engineers, Part I: Journal of Systems and Control Engineering, vol. 225, pp. 954-967, Nov. 2011.

[122] K. Lee and D. Y. Lee, "Adjusting output-limiter for stable haptic interaction with deformable objects," in Control Automation, 200\%. MED '0\%. Mediterranean Conference on, pp. 1-6, 2007.

[123] B. Hannaford and J.-H. Ryu, "Time-domain passivity control of haptic interfaces," Robotics and Automation, IEEE Transactions on, vol. 18, pp. 1 -10, Feb. 2002.

[124] J.-H. Ryu, C. Preusche, B. Hannaford, and G. Hirzinger, "Time domain passivity control with reference energy following," Control Systems Technology, IEEE Transactions on, vol. 13, no. 5, pp. 737-742, 2005.

[125] M. Dohring and W. Newman, "The passivity of natural admittance control implementations," in Robotics and Automation, 2003. Proceedings. ICRA '03. IEEE International Conference on, vol. 3, pp. 3710 - 3715 vol.3, sept. 2003.

[126] S. Stramigioli, C. Secchi, A. vanderSchaft, and C. Fantuzzi, "Sampled data systems passivity and discrete port-hamiltonian systems," Robotics, IEEE Transactions on, vol. 21 , pp. $574-587$, aug. 2005.

[127] M.-C. Chien and A.-C. Huang, "Adaptive impedance control of robot manipulators based on function approximation technique," Robotica, vol. 22, no. 4, pp. 395-403, 2004. 
[128] S. H. Kang, M. Jin, and P. H. Chang, "A solution to the accuracy/robustness dilemma in impedance control," Mechatronics, IEEE/ASME Transactions on, vol. 14, pp. 282294 , june 2009 .

[129] A. Abdossalami and S. Sirouspour, "Adaptive control for improved transparency in haptic simulations," Haptics, IEEE Transactions on, vol. 2, pp. 2 -14, jan.-march 2009 .

[130] S. PChan and H. PChen, "Robust implementation of generalized impedance control for robot manipulators," Advanced Robotics, vol. 15, no. 6, pp. 641-661, 2001.

[131] C.-C. Cheah and D. Wang, "Learning impedance control for robotic manipulators," Robotics and Automation, IEEE Transactions on, vol. 14, pp. 452 -465, jun 1998.

[132] W. Yu, J. Rosen, and X. Li, "Pid admittance control for an upper limb exoskeleton," in American Control Conference (ACC), 2011, pp. 1124-1129, 29 2011-july 12011.

[133] Y. Li, S. S. Ge, C. Yang, X. Li, and K. P. Tee, "Model-free impedance control for safe human-robot interaction," in Robotics and Automation (ICRA), 2011 IEEE International Conference on, pp. 6021 -6026, may 2011.

[134] M. V. Kothare, P. J. Campo, M. Morari, and C. N. Nett, "A unified framework for the study of anti-windup designs," Automatica, vol. 30, pp. 1869-1883, 1994.

[135] F. Morabito, A. Teel, and L. Zaccarian, "Nonlinear antiwindup applied to eulerlagrange systems," Robotics and Automation, IEEE Transactions on, vol. 20, pp. 526 - 537, june 2004.

[136] J. J. G. Inaki Diaz and T. Hulin, "Stability boundary and transparency for haptic rendering," Advances in Haptics, April 2010.

[137] K. Eom, I. Suh, and B.-J. Yi, "A design method of a haptic interface controller considering transparency and robust stability," in Intelligent Robots and Systems, 2000. (IROS 2000). Proceedings. 2000 IEEE/RSJ International Conference on, vol. 2, pp. $961-966$ vol.2, 2000.

[138] E. de Vlugt, A. C. Schouten, F. C. van der Helm, P. C. Teerhuis, and G. G. Brouwn, "A force-controlled planar haptic device for movement control analysis of the human arm," Journal of Neuroscience Methods, vol. 129, pp. 151-168, Oct. 2003.

[139] V. Duchaine, B. Mayer St.-Onge, D. Gao, and C. Gosselin, "Stable and intuitive control of an intelligent assist device," Haptics, IEEE Transactions on, vol. 5, pp. 148 -159 , april-june 2012 .

[140] A. Morbi, M. Ahmadi, A. D. C. Chan, and R. Langlois, "Comparing continuous and intermittent assistance controllers for assistive devices," in Robotics and Automation (ICRA), 2011 IEEE International Conference on, pp. 4651-4656, 2011. 
[141] G. Aguirre-Ollinger, J. Colgate, M. Peshkin, and A. Goswami, "Inertia compensation control of a one-degree-of-freedom exoskeleton for lower-limb assistance: Initial experiments," Neural Systems and Rehabilitation Engineering, IEEE Transactions on, vol. 20, pp. $68-77$, Jan. 2012.

[142] H. K. Khalil, Nonlinear Systems (3rd Edition). Prentice Hall, 2001.

[143] D. Liberzon, Switching in systems and control. Springer, 2003.

[144] A. Morbi, "Assistance regulation in wearable robots," Master's thesis, Carleton University, 2009.

[145] A. Bemporad, "Reference governor for constrained nonlinear systems," Automatic Control, IEEE Transactions on, vol. 43, pp. 415-419, mar 1998.

[146] E. Gilbert and I. Kolmanovsky, "Nonlinear tracking control in the presence of state and control constraints: a generalized reference governor," Automatica, vol. 38, no. 12, pp. $2063-2073,2002$.

[147] C. Wen, J. Zhou, Z. Liu, and H. Su, "Robust adaptive control of uncertain nonlinear systems in the presence of input saturation and external disturbance," Automatic Control, IEEE Transactions on, vol. 56, pp. 1672-1678, july 2011.

[148] E. Scholte and M. Campbell, "Robust nonlinear model predictive control with partial state information," Control Systems Technology, IEEE Transactions on, vol. 16, pp. 636-651, july 2008.

[149] J. Gil, A. Rubio, and J. Savall, "Decreasing the apparent inertia of an impedance haptic device by using force feedforward," Control Systems Technology, IEEE Transactions on, vol. 17, pp. $833-838$, july 2009.

[150] D. E. Miller, "A new approach to adaptive control: no nonlinearities," Systems $\&$ Control Letters, vol. 49, no. 1, pp. 67 - 79, 2003. ¡ce:title¿Adaptive Controli/ce:title¿.

[151] B. Hannaford and J.-H. Ryu, "Time-domain passivity control of haptic interfaces," Robotics and Automation, IEEE Transactions on, vol. 18, no. 1, pp. 1-10, 2002.

[152] J.-P. Kim and J. Ryu, "Robustly stable haptic interaction control using an energybounding algorithm," The International Journal of Robotics Research, vol. 29, no. 6, pp. 666-679, 2010.

[153] Y. Ye, Y.-J. Pan, Y. Gupta, and J. Ware, "A power-based time domain passivity control for haptic interfaces," Control Systems Technology, IEEE Transactions on, vol. 19, no. 4, pp. 874-883, 2011.

[154] J.-P. Kim, S. yun Baek, and J. Ryu, "A force bounding approach for stable haptic interaction," in World Haptics Conference (WHC), 2011 IEEE, pp. 397-402, 2011. 
[155] M. Franken, S. Stramigioli, S. Misra, C. Secchi, and A. Macchelli, "Bilateral telemanipulation with time delays: A two-layer approach combining passivity and transparency," Robotics, IEEE Transactions on, vol. 27, no. 4, pp. 741-756, 2011.

[156] E. Steur, I. Tyukin, and H. Nijmeijer, "Semi-passivity and synchronization of diffusively coupled neuronal oscillators," Physica D: Nonlinear Phenomena, vol. 238, no. 21, pp. $2119-2128,2009$.

[157] H. G. Sage, M. F. De Mathelin, and E. Ostertag, "Robust control of robot manipulators: A survey," International Journal of Control, vol. 72, no. 16, pp. 1498-1522, 1999.

[158] R. Kikuuwe, "Alternative proofs of four stability properties of rigid-link manipulators under pid position control," Robotica, vol. 31, pp. 113-122, 12013.

[159] A. Pervozvanski and L. Freidovich, "Robust stabilization of robotic manipulators by pid controllers," Dynamics and Control, vol. 9, no. 3, pp. 203-222, 1999.

[160] Z. Qu, "Global stability of trajectory tracking of robot under pd control," in Control Applications, 1992., First IEEE Conference on, pp. 8-12 vol.1, 1992.

[161] J. Alvarez-Ramirez, R. Kelly, and I. Cervantes, "Semiglobal stability of saturated linear pid control for robot manipulators," Automatica, vol. 39, no. 6, pp. 989 - 995, 2003.

[162] H. Lim, K. Hoon, K. Low, Y. Soh, and A. Tow, "Pelvic control and over-ground walking methodology for impaired gait recovery," in Robotics and Biomimetics, 2008. ROBIO 2008. IEEE International Conference on, pp. 282 -287, feb. 2009.

[163] H. Lim, K. H. Hoon, K. H. Low, Y. Soh, and A. Tow, "Pelvic control and over-ground walking methodology for impaired gait recovery," in Robotics and Biomimetics, 2008. ROBIO 2008. IEEE International Conference on, pp. 282-287, 2009.

[164] P. P. Avi Nativ. Personal Conversation.

[165] K. A. Bieryla and M. L. Madigan, "Proof of concept for perturbation-based balance training in older adults at a high risk for falls," Archives of physical medicine and rehabilitation, vol. 92, no. 5, pp. 841-843, 2011.

[166] L. B. Luciani, V. Genovese, V. Monaco, L. Odetti, E. Cattin, S. Micera, et al., "Design and evaluation of a new mechatronic platform for assessment and prevention of fall risks," Journal of neuroengineering and rehabilitation, vol. 9, no. 1, p. 51, 2012.

[167] A. Shapiro and I. Melzer, "Balance perturbation system to improve balance compensatory responses during walking in old persons," Journal of NeuroEngineering and Rehabilitation, vol. 7, no. 1, p. 32, 2010. 
[168] R. Holmberg and O. Khatib, "Development and control of a holonomic mobile robot for mobile manipulation tasks," I. J. Robotic Res., vol. 19, no. 11, pp. 1066-1074, 2000 .

[169] H. Yu, M. Spenko, and S. Dubowsky, "Omni-directional mobility using active split offset castors," Journal of Mechanical Design, vol. 126, no. 5, pp. 822-829, 2004.

[170] K. Watanabe, Y. Shiraishi, S. G. Tzafestas, J. Tang, and T. Fukuda, "Feedback control of an omnidirectional autonomous platform for mobile service robots," Journal of Intelligent Éamp; Robotic Systems, vol. 22, pp. 315-330, 1998. 10.1023/A:1008048307352.

[171] R. Damoto, W. Cheng, and S. Hirose, "Holonomic omnidirectional vehicle with new omni-wheel mechanism," vol. 1, pp. 773 - 778 vol.1, 2001.

[172] G. Indiveri, "Swedish wheeled omnidirectional mobile robots: Kinematics analysis and control," Robotics, IEEE Transactions on, vol. 25, pp. 164 -171, feb. 2009.

[173] M. West and H. Asada, "Design of a holonomic omnidirectional vehicle," pp. $97-103$ vol.1, may. 1992.

[174] M. Wada, "Holonomic and omnidirectional wheelchairs with synchronized 4wd mechanism," in Intelligent Robots and Systems, 200\%. IROS 200\%. IEEE/RSJ International Conference on, pp. 1196 -1202, 292007-nov.2 2007.

[175] M. Mariappan, C. C. Wee, K. Vellian, and C. K. Weng, "A navigation methodology of an holonomic mobile robot using optical tracking device (otd)," in TENCON 2009 - 2009 IEEE Region 10 Conference, pp. 1 -6, 2009.

[176] D. Y. Kim and K. Y. Yi, "A user-steered guide robot for the blind," in Robotics and Biomimetics, 2008. ROBIO 2008. IEEE International Conference on, pp. 114 -119, 2009.

[177] M. Killpack, T. Deyle, C. Anderson, and C. C. Kemp, "Visual odometry and control for an omnidirectional mobile robot with a downward-facing camera," pp. $139-146$, 2010.

[178] C.-C. Wong, Y.-H. Lin, S.-A. Lee, and C.-H. Tsai, "Ga-based fuzzy system design in fpga for an omni-directional mobile robot," Journal of Intelligent Éamp; Robotic Systems, vol. 44, pp. 327-347, 2005. 10.1007/s10846-005-9020-6.

[179] Rotacaster. http://www.rotacaster.com.au/omnidirectional-wheels-mounts.html.

[180] C. Ward and K. Iagnemma, "A dynamic-model-based wheel slip detector for mobile robots on outdoor terrain," Robotics, IEEE Transactions on, vol. 24, no. 4, pp. 821 $-831,2008$. 
[181] J. M. Potter, A. L. Evans, and G. Duncan, "Gait speed and activities of daily living function in geriatric patients," Archives of Physical Medicine and Rehabilitation, vol. 76 , no. 11, pp. $997-999,1995$.

[182] MathWorks, "Support documentation." http://www.mathworks.com/help/index.html.

[183] A. Morbi, M. Ahmadi, and A. Nativ, "Gaitenable: An omnidirectional robotic system for gait rehabilitation," in Mechatronics and Automation (ICMA), 2012 International Conference on, pp. 936-941, 2012.

[184] Y. Yamamoto, Control and Coordination of Locomotion and Manipulation of a Wheeled Mobile Manipulator. PhD thesis, University of Pennsylvania, 1994.

[185] J. S. Gottschall and R. Kram, "Energy cost and muscular activity required for leg swing during walking.," J Appl Physiol, vol. 99, pp. 23-30, Jul 2005.

[186] T. Kalmar-Nagy, P. Ganguly, and R. D'Andrea, "Real-time trajectory generation for omnidirectional vehicles," in American Control Conference, 2002. Proceedings of the 2002, vol. 1, pp. 286 - 291 vol.1, 2002.

[187] L. Paz, J. Tardos, and J. Neira, "Divide and conquer: Ekf slam in," Robotics, IEEE Transactions on, vol. 24, pp. $1107-1120$, oct. 2008.

[188] D. Lhomme-Desages, C. Grand, J.-C. Guinot, and F. Ben Amar, "Doppler-based ground speed sensor fusion and slip control for a wheeled rover," Mechatronics, IEEE/ASME Transactions on, vol. 14, pp. 484 -492, aug. 2009.

[189] C. Ward and K. Iagnemma, "A dynamic-model-based wheel slip detector for mobile robots on outdoor terrain," Robotics, IEEE Transactions on, vol. 24, pp. $821-831$, aug. 2008.

[190] C. P. LLC, "Rifton tram." http://www.rifton.com/products/lift-and-transferdevices/rifton-tram. 


\section{Appendix A}

\section{The Mobile Base Jacobian}

For the wheel configuration used during the experiments described in Chapters 4 and 5, the exact expression of $J^{-1}$ was determined to be

$$
J^{-1}=\left[\begin{array}{ccc}
20.80 & 0.00 & 17.24 \\
4.18 & 12.1915 & -12.19 \\
4.18 & -12.195 & -12.19
\end{array}\right] \text {. }
$$

